\title{
Probing Flavor Asymmetry of Anti-quarks in the Proton by Drell-Yan Experiment SeaQuest
}

\author{
Shou Miyasaka
}

Thesis submitted to the Department of Physics
for the degree of
Doctor of Science at Tokyo Institute of Technology

August, 2016 


\begin{abstract}
A new measurement on the flavor asymmetry between $\bar{d}$ and $\bar{u}$ in the proton is reported in this thesis. The proton contains a substantial number of antiquarks which arise from dynamical interactions of gluons such as gluon dissociation to a quark-antiquark pair, $g \rightarrow q+\bar{q}$, and from non-perturbative processes as described by the pion-cloud model, for example. The antiquarks in the proton undertake an important role in determining the dynamic characteristics of the internal structure of the proton, although its distribution in the proton and its origin are not fully understood. Understanding sea quarks in hadron is an important subject for QCD.

The SeaQuest experiment at Fermi National Accelerator Laboratory (Fermilab) is a fixed target experiment using the $120 \mathrm{GeV}$ proton beam extracted from the Fermilab Main Injector. One of the goals of the experiment is to measure the flavor asymmetry between $\bar{d}$ quark and $\bar{u}$ quark in the proton as a function of the target Bjorken $x$ using the Drell-Yan process in the $p-p$ or $p-d$ reactions. This process takes place in hadron-hadron collisions when a quark in one hadron in the beam and an antiquark in other hadron in the target annihilate into a virtual photon that decays into a lepton pair.

The flavor asymmetry between $\bar{d}$ and $\bar{u}$ quarks was found by deep-inelastic scattering experiment NMC at CERN. The E866/NuSea experiment at Fermilab obtained the flavor asymmetry in the proton for $0.015<x<0.35$ using the $800 \mathrm{GeV}$ proton beam extracted from the Fermilab Tevatron. The result indicates the dominance of $\bar{d}$; it is $70 \%$ larger than $\bar{u}$ at lower $x$. The SeaQuest experiment was planned to do a new precise measurement at higher $x$ region. The lower energy beam $(120 \mathrm{GeV})$ increases the Drell-Yan cross section and suppresses the background primarily arising from $\mathrm{J} / \psi$ decays. Therefore, SeaQuest will obtain more statistics in a shorter time than the E866 experiment.

After detector construction, detector commissioning and accelerator upgrade, physics data taking started in 2013. The SeaQuest spectrometer is designed to detect dimuon from the Drell-Yan process. It consists of targets, two di-pole magnets, and four tracking detector groups. The third tracking detector group has two drift chambers. One was newly fabricated in Japan by the Japanese group in SeaQuest collaboration and was shipped to Fermilab. The other one was constructed by SeaQuest collaborator in Fermilab under the initiative of the Japanese group.

I worked on the construction and installation of the detectors, data taking and data analysis in SeaQuest. I extracted the flavor asymmetry as a function of Bjorken $x$ using
\end{abstract}


the SeaQuest data for the first time. This thesis shows the results using a part of data taken in 2014 and 2015. The asymmetry was extracted for much wider Bjorken $x$ region than the previous experiment. The measured Bjorken $x$ range covers up to 0.58. The result shows that the ratio of $\bar{d} / \bar{u}$ is always higher than 1 at $0.1<x<0.45$, in contrast to the E866 result. For $0.45<x<0.58$, the result shows that the ratio is close to unity.

Predictions made by current PDF parameterizations are in agreement with the present result. Also, a prediction obtained by one of the non-perturbative models, pion-cloud model, is closer to the SeaQuest result than the E866 result. This result of $\bar{d} / \bar{u}$ asymmetry at the wide Bjorken $x$ region, $0.1<x<0.58$, is very important information to understand the inner structure of the proton and the origin of the sea quarks in the proton. 


\section{Contents}

1 Introduction 5

2 Physics and Motivation $\quad 9$

2.1 Internal Structure of the Proton . . . . . . . . . . . . . . . 9 9

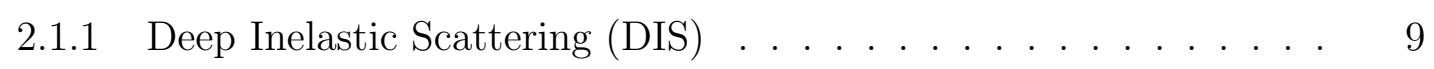

2.1 .2 Quark-Parton Model . . . . . . . . . . . . . . . . . . . . . . . 10

2.2 Quantum Chromodynamics (QCD) . . . . . . . . . . . . . . 11

2.3 Drell-Yan Process . . . . . . . . . . . . . . . . . . . . 14

$2.3 .1 \quad$ Cross section for the Drell-Yan process . . . . . . . . . . . . . . 14

2.3 .2 Kinematics of the Drell-Yan process $\ldots \ldots \ldots$

3 Review of Experiments on Flavor Asymmetry of Anti-quark Sea 16

3.1 NMC experiment . . . . . . . . . . . . . . . . 16

3.2 NA51 experiment . . . . . . . . . . . . . . . . . . . . . . . . . . . 17

3.3 E866/NuSea experiment . . . . . . . . . . . . . . . . . . . . . . . . . 19

3.4 Motivation for the SeaQuest Experiment . . . . . . . . . . . . . . . . 21

B.5 Calculation of kinematics . . . . . . . . . . . . . . . . . . . . . 21

$3.6 \quad$ Future $d / \bar{u}$ experiments $\ldots \ldots \ldots \ldots \ldots$. . . . . . . . . . . . . . . 21

4 Experimental Method: SeaQuest 23

4.1 Accelerator and Beam . . . . . . . . . . . . . . . . . 27

4.2 Beam Monitor . . . . . . . . . . . . . . . . . . . . . . . . . . 29

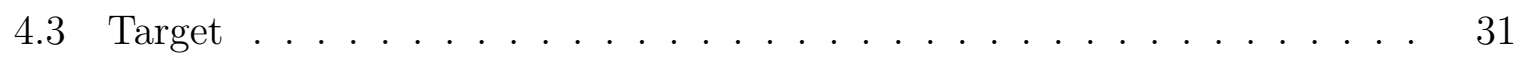

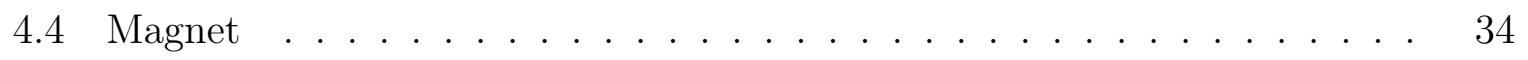

4.5 Detector stations . . . . . . . . . . . . . . . . . . 37

4.5 .1 Scintillator Hodoscopes . . . . . . . . . . . . . . . . . . . . . 37

4.5 .2 Drift Chambers . . . . . . . . . . . . . . . . 43

$4.5 .3 \quad$ Structure of SeaQuest drift chambers . . . . . . . . . . . . . . . 47 
4.5 .4 Proportional Tubes $\ldots \ldots \ldots$. . . . . . . . . . . . . . . . 53

4.6 Readout System for Detectors . . . . . . . . . . . . . . . . . 55

$4.6 .1 \mathrm{TDC}$ card . . . . . . . . . . . . . . . . . . 55

$4.6 .2 \quad \mathrm{ASDQ}$ card $\ldots \ldots \ldots \ldots \ldots$

4.6 .3 Level shifter board . . . . . . . . . . . . . 56

4.7 Monte Carlo simulation . . . . . . . . . . . . . . . . . . . . 60

4.8 Detector acceptance $\ldots \ldots \ldots$. . . . . . . . . . . . . . . . . . . . . 61

4.9 Trigger . . . . . . . . . . . . . . . . . . . . . . . . . . . . . . 63

4.9 .1 FPGA trigger . . . . . . . . . . . . . . . . . . 64

4.9 .2 NIM trigger . . . . . . . . . . . . . . . . . . . . 65

4.9 .3 Roadset in physics run . . . . . . . . . . . . . . . . . . 66

4.10 Data Acquisition System . . . . . . . . . . . . . . . . . . . . . 68

4.10 .1 Main DAQ . . . . . . . . . . . . . . . . . . . . . . 68

4.10 .2 Scaler DAQ $\ldots \ldots \ldots \ldots$

4.10 .3 Beam DAQ . . . . . . . . . . . . . . . . . . . . . . . . . . . . . 71

4.11 Decoder and production . . . . . . . . . . . . . . . . 71

4.12 Fabrication of Station 3 minus drift chamber . . . . . . . . . . . . . . . . 71

4.12 .1 Wire assembling . . . . . . . . . . . . . . . . . . . . . . . 73

4.12 .2 Tension measurement . . . . . . . . . . . . . . . . 73

$\begin{array}{lll}5 \text { Data Analysis } & 77\end{array}$

5.1 Data sets . . . . . . . . . . . . . . . . . . 77

5.2 Spill Selection . . . . . . . . . . . . . . . . . . . . . . . 77

5.2 .1 Applied spill cuts . . . . . . . . . . . . . . . . . . . . . . . . . . . . . . . . . . . . . . . . .

5.2 .2 Results of spill cuts . . . . . . . . . . . . . . . . . . 86

5.3 Dimuon reconstruction . . . . . . . . . . . . . . . . . . . 87

5.3 .1 Pre-tracking analysis . . . . . . . . . . . . . . . 87

5.3 .2 Track reconstruction . . . . . . . . . . . . . . . . . 88

5.3 .3 vertex fitting . . . . . . . . . . . . . . . . . . . . . 92

5.4 Track selection . . . . . . . . . . . . . . . . . . . . . . . . 94

5.5 Dimuon selection . . . . . . . . . . . . . . . . . . . . 94

5.5 .1 Kinematics cuts . . . . . . . . . . . . . . . . . . . . 94

5.5 .2 Target-dump separation $\ldots \ldots \ldots$. . . . . . . . . 96

5.6 Dimuon yield $\ldots \ldots \ldots$

5.7 Basic information of dimuon events . . . . . . . . . . . . . . . . . 101

5.7 .1 Mass distribution . . . . . . . . . . . . . . . . . . . . 101 
5.7 .2 Bjorken $x$ distribution $\ldots \ldots \ldots \ldots \ldots \ldots$

5.7 .3 z-vertex distribution . . . . . . . . . . . . . . . . . . 104

5.7 .4 Hit distribution on chamber plane . . . . . . . . . . . . 107

5.7 .5 Occupancy of chamber plane. . . . . . . . . . . . . . . 107

5.7 .6 Track position on chamber plane . . . . . . . . . . . 107

5.8 Drift chamber performance . . . . . . . . . . . . . . . . . . 111

5.8 .1 K-T curve . . . . . . . . . . . . . . . . . . . . . . 111

5.8 .2 Chamber efficiency . . . . . . . . . . . . . . . . . . . 117

5.9 Extraction of Cross Section Ratid . . . . . . . . . . . . . . . . 119

5.9 .1 Number of proton per RF bucket . . . . . . . . . . . . . . 119

5.9 .2 "Trigger intensity" and "Chamber intensity" . . . . . . . . . 120

5.9 .3 Intensity dependence correction . . . . . . . . . . . . . . 124

5.9.4 Intensity dependence of combinatorial background . . . . . 130

5.9 .5 Cut on Chamber Intensity . . . . . . . . . . . . . . . . 130

5.9 .6 Target contamination correction . . . . . . . . . . . . . . . 134

5.97 Formula of cross section ratid . . . . . . . . . . . . 136

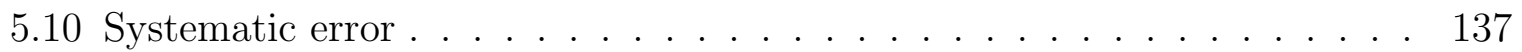

5.10 .1 Correction of intensity dependence . . . . . . . . . . . 137

5.10 .2 Intensity dependence on cross section ratio . . . . . . . . . . 139

5.10 .3 Correction of target contamination $\ldots \ldots \ldots \ldots \ldots$

5.10 .4 Target length . . . . . . . . . . . . . . . . . . . . . . 141

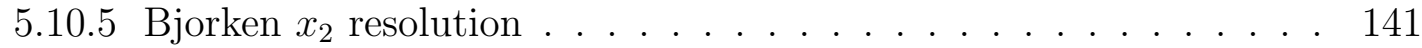

5.10 .6 Beam intensity $\ldots \ldots \ldots \ldots \ldots \ldots$. . . . . . . . . . 144

5.10 .7 Total systematic error $\ldots \ldots \ldots \ldots \ldots$

5.10 .8 Result of Cross section ratid . . . . . . . . . . . . . . 147

5.11 Extraction of $d / \bar{u} \ldots \ldots \ldots \ldots \ldots \ldots$

5.11 Prediction of cross section ratid . . . . . . . . . . 150

5.11 .2 Adjustment of $d / \vec{u} \ldots \ldots \ldots \ldots \ldots \ldots$

5.11 .3 Result of $d / \bar{u} \ldots \ldots \ldots \ldots \ldots \ldots \ldots$

6 Discussion of the Results $\quad 159$

6.1 Cross section ratid . . . . . . . . . . . . . . . . . . . . . . . . 159

$6.2 \quad$ Flavor asymmetry . . . . . . . . . . . . . . . . . . . . . . . . . . . . 164

6.2 .1 Comparison to other experiments . . . . . . . . . . . . 164

$6.2 .2 \quad$ PDF parameterizations $\ldots \ldots \ldots \ldots \ldots$

6.2 .3 Possible origins of the sea quark in the proton . . . . . . . 168 
7 Conclusion and Future Prospect 170

7.1 Conclusion . . . . . . . . . . . . . . . . . . . . 170

7.2 Future prospect $\ldots \ldots \ldots \ldots \ldots \ldots$. . . . . . . . . . . . . . . . . 171

7.3 Summary . . . . . . . . . . . . . . . . . . . . . 173 


\section{Chapter 1}

\section{Introduction}

The universe and the matter are constructed by a number of elementary particles which interact with fundamental forces. The ultimate goal of the Particle Physics is to understand the structure of the matter and explain the characteristics of all the elementary particles and their interactions[1], 2, 3]. The matter we interact with every day is made of protons, neutrons and electrons. The electron is known as a fundamental particle, namely it doesn't have internal structure in it. On the other hand, it was found that the proton has a spacial distribution and internal structure by measuring its form factor using electron-proton scattering in 1950s. Later, a quark in the proton was found using the Deep Inelastic Scattering (DIS), $e+p \rightarrow e^{\prime}+X$, by experiments at SLAC[4, 5]. The DIS is one of the successful tools to investigate the internal structure of the proton. Afterward Quark-Parton model was developed to describe the observations of the experiment. In this model, the proton consists of three valence quarks as shown in the left figure of Fig. ㅁ.

Static characteristics of the proton such as mass and charge are successfully explained with these quarks. However, more dynamical structure was observed in high energy interactions; the valence quarks are glued by "gluons", and the gluon produces a pair of quark and anti-quark, $g \rightarrow d \bar{d}$ or $g \rightarrow u \bar{u}$, then they annihilate back to a gluon after a short time as shown in the center- and right- figures of Fig. ㄴ. 1 . Those quarks and anti-quarks are called "sea" quarks. It is important to know the characteristics of the sea quarks in light of Quantum ChrmoDynamics (QCD). QCD was developed in 1960's. The asymptotic freedom[7] was theoretically found. The physical meaning of Bjorken $x$ became clear. Theoretical foundation for parton distributions and their $Q^{2}$ evolution were established.

The New Muon Collaboration (NMC) [8, , $]$ studied the proton structure using DIS. They observed the flavor asymmetry of anti-quark distributions in the proton, and discov- 


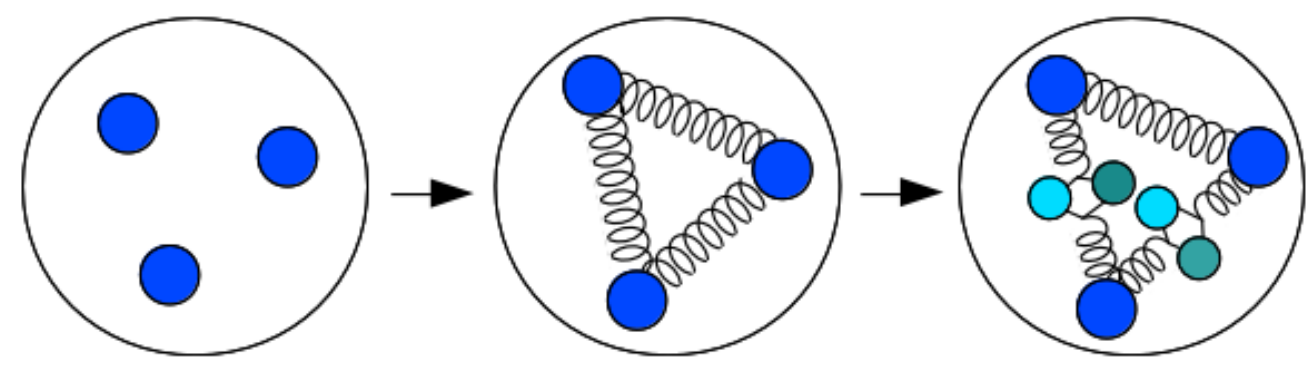

Figure 1.1: Transition of the proton model. Quark-Parton model describes that the proton consists of three valence quarks as shown in the left figure. Later, more dynamical structure was observed in high energy interactions; the valence quarks are glued by gluon as shown in the center figure, and the gluon produces a pair of quark and anti-quark as shown in the right figure.

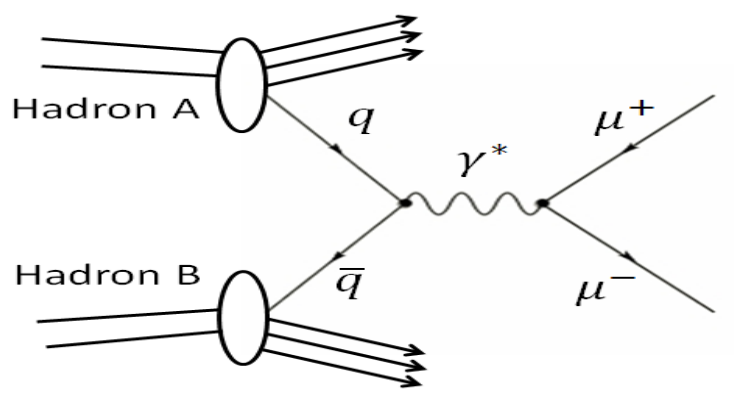

Figure 1.2: Kinematics of the Drell-Yan process. The process is a hadron A - hadron B collision in which a quark in one hadron and an anti-quark in the other hadron annihilate into a virtual photon and then a muon pair is created.

ered that the proton has more $\bar{d}$ than $\bar{u}$. The NA51 experiment[IIV] used the $450 \mathrm{GeV} / \mathrm{c}$ $(\sqrt{s} \sim 29 \mathrm{GeV})$ proton beam from the CERN Synchrotoron Proton Source to measure the asymmetry between $\bar{d}$ and $\bar{u}$ using Drell-Yan process[1]]. The Drell-Yan process is a good tool to study anti-quarks in the proton. The Drell-Yan process takes place in hadron-hadron scattering. In the leading order of this process, a quark from one hadron and an anti-quark from the other hadron annihilate into a virtual photon, and then it decays into di-lepton is seen in Fig. [1.2. The Drell-Yan process is well suited to measure the anti-quark distributions of the proton, since an anti-quark is always involved in this process.

The E866/NuSea[12, 13, 14] experiment at Fermi National Accelerator Laboratory (Fermilab) was a fixed-target Drell-Yan experiment. This experiment measured the ratio of $\bar{d}(x) / \bar{u}(x)$ in the proton at $0.015<x<0.35$ while the NA51 experiment measured 
it only at $x=0.18$. The E866 experiment used the $800 \mathrm{GeV} / c$ proton beam extracted from the Fermilab Tevatron accelerator. From the result of E866 experiment, shown in Fig. $\square .3$, the ratio of $\bar{d}(x) / \bar{u}(x)$ is as large as 1.7 . Also, the ratio seems to be smaller than 1 at high $x(>0.3)$ although it is not yet conclusive due to the limited statistical accuracy. This is an indication of new characteristics of the proton structure, because currently no theoretical models can reproduce this behavior. So, it is very important to measure the ratio at higher Bjorken $x$.

SeaQuest (E906) experiment[15] at Fermilab was planned to do a new measurement of the ratio $\bar{d}(x) / \bar{u}(x)$ at $0.1<x<0.45$ as shown in Fig. ㄴ.3]. SeaQuest uses an $120 \mathrm{GeV}$ proton beam from the Main Injector at Fermilab while the E866 experiment used the $800 \mathrm{GeV}$ proton beam. The lower energy beam $(120 \mathrm{GeV})$ increases the Drell-Yan cross section and suppresses the background which primarily comes from $\mathrm{J} / \psi$ decays. Therefore, SeaQuest will obtain more statistics in shorter time than the E866 experiment.

This thesis is structured as follows: In Chapter 2, physics theory which are relevant for the understanding of the Drell-Yan measurement are described. In Chapter 3, the history of the investigation of the flavor asymmetry of anti-quark distributions and the methods of the study are described. In Chapter 4, the main components of the SeaQuest apparatus are explained. Proton beam, targets, magnets, detectors, data acquisition systems and related electronics are described. Chapter 5 is the main part which describes the details of data analysis. The cross section ratio, $\sigma^{p d} / 2 \sigma^{p p}$, and the flavor asymmetry of $\bar{d} / \bar{u}$ using the SeaQuest data are presented. The results are compared with the results from other experiments in Chapter 6. Chapter 7 then shows conclusions. 

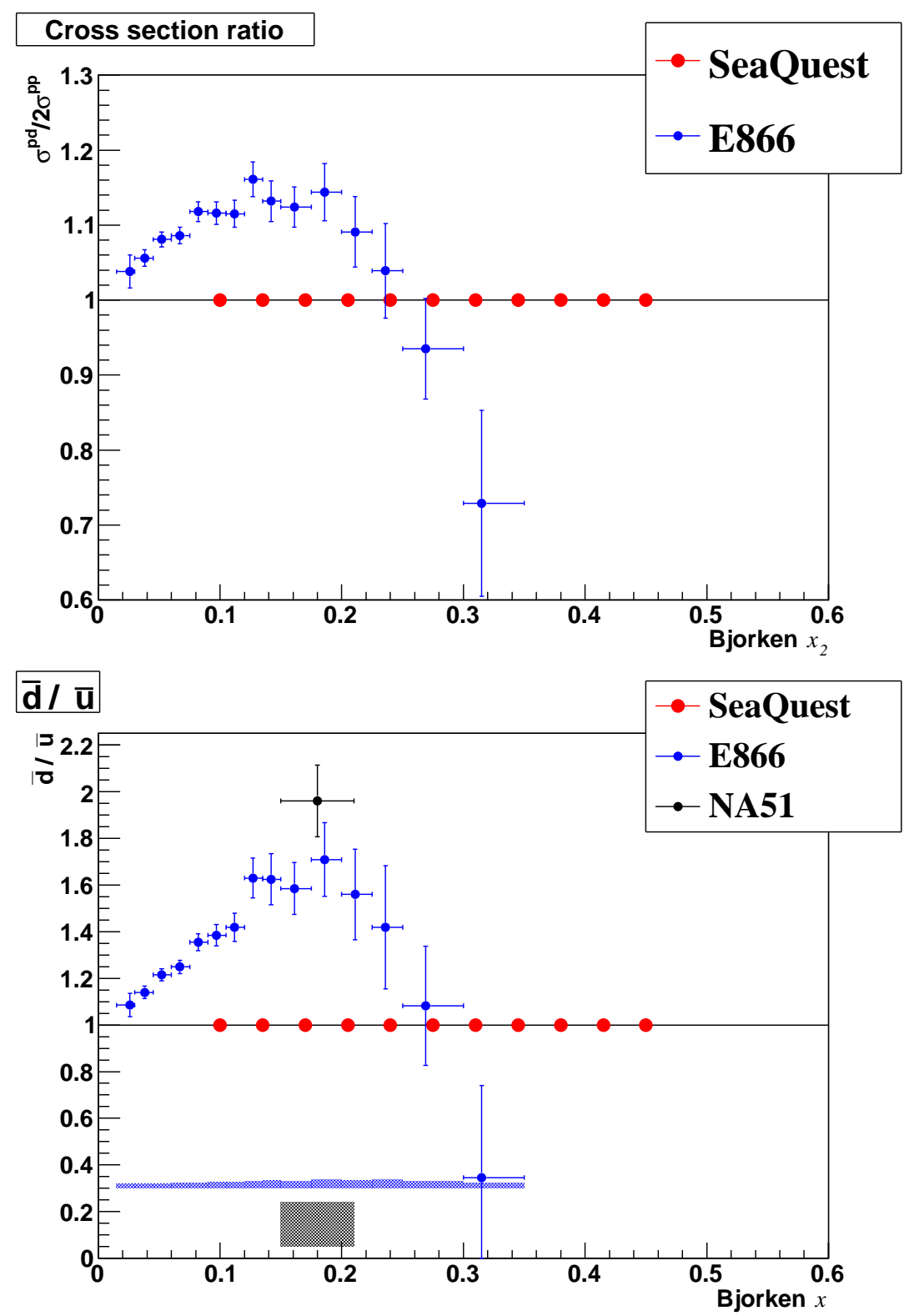

Figure 1.3: Results of the E866 experiment performed at Fermilab. The top figure shows the ratio of $\sigma^{p d} / 2 \sigma^{p p}$, and the bottom figure shows $\bar{d}(x) / \bar{u}(x)$ [14]. The E866 results are plotted in blue. The error bar indicates statistical error and the error band indicates systematic error. The expected Bjorken $x$ range for the SeaQuest measurement are plotted with red points (arbitrarily plotted at 1.0). Also, the result of the NA51 experiment is shown in the $\bar{d}(x) / \bar{u}(x)$ figure in black. 


\section{Chapter 2}

\section{Physics and Motivation}

\subsection{Internal Structure of the Proton}

\subsubsection{Deep Inelastic Scattering (DIS)}

The deep inelastic scattering (DIS) is a successful tool to investigate the internal structure of the proton. In lepton-proton scattering, a lepton $l$ interacts with a proton $P$ through the exchange of a virtual boson as shown in Fig. 2.]. We are able to investigate the properties and the substructure of the proton by measuring the kinematic distributions of the scattered lepton. At high energy, virtual weak boson, such as $W^{ \pm}, Z^{0}$, can be exchanged in the scattering. However, the electromagnetic interaction through the exchange of a virtual photon is dominant due to the large masses of those weak bosons, and the weak interaction is negligible in the kinematic conditions of the SeaQuest experiment. The kinematic variables related to the DIS are listed in Tab. 2.].

Table 2.1: Kinematic variables in DIS

\begin{tabular}{ll}
\hline \hline$k=(E, \mathbf{k}), k^{\prime}=\left(E^{\prime}, \mathbf{k}^{\prime}\right)$ & 4-momenta of the initial- and final-state leptons \\
$P=(M, \mathbf{0})$ & 4-momentum of the initial target proton \\
$q=k-k^{\prime}$ & 4-momentum of the virtual photon \\
$W=P+q$ & 4-momentum of the final state proton \\
$\nu=E-E^{\prime}$ & Energy of the virtual photon \\
$\theta$ & Scattering angle of the lepton \\
$\Omega$ & Solid angle of scattered lepton detection \\
$x=\frac{Q^{2}}{2 P \cdot q}=\frac{Q^{2}}{2 M \nu}$ & Bjorken scaling variable \\
$y=\frac{\nu}{E}$ & Fractional energy of the virtual photon \\
$s=(k+P)^{2}$ & The center-of-mass energy squared of the lepton-proton system \\
$Q^{2}=-q^{2}$ & The momentum transfer squared \\
\hline
\end{tabular}




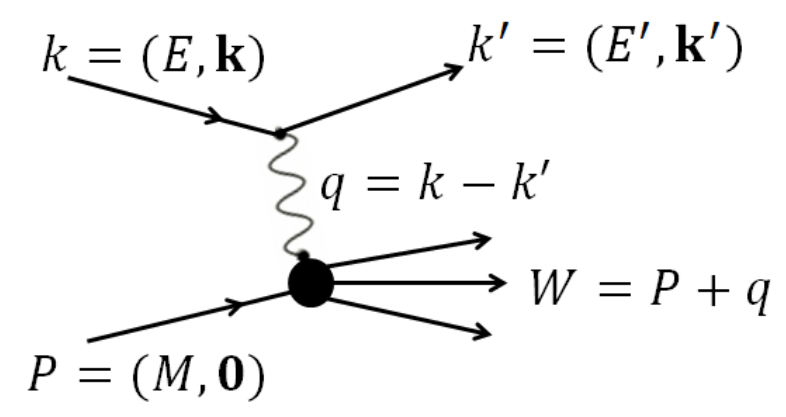

Figure 2.1: Schematic drawing of DIS. $k, k^{\prime}$ are 4-momenta of the initial- and final-state leptons. $P$ is the 4-momentum of the initial target proton, and $M$ is the mass of the proton. $q$ is the 4 -momentum of the virtual photon. $W$ is the 4-momentum of the final state hadrons. Energy of the virtual photon $\nu$ is defined as $\nu=E-E^{\prime}$.

The differential cross section of DIS can be expressed using the structure functions $F_{1}\left(x, Q^{2}\right)$ and $F_{2}\left(x, Q^{2}\right)$ as

$$
\frac{d^{2} \sigma}{d \Omega d E^{\prime}}=\frac{\alpha^{2}}{4 E^{\prime} \sin ^{4} \frac{\theta}{2}}\left[\frac{1}{\nu} F_{2}\left(x, Q^{2}\right) \cos ^{2} \frac{\theta}{2}+\frac{1}{M} F_{1}\left(x, Q^{2}\right) \sin ^{2} \frac{\theta}{2}\right],
$$

where $E^{\prime}$ is the final state energy of the scattered lepton, $\theta$ the angle of the scattered lepton in the laboratory frame, $\nu$ the energy of the virtual photon, $M$ the mass of the target proton, $Q^{2}$ the momentum transfer squared, and $x$ the Bjorken scaling. Here, $Q^{2}$ is equal to $-q^{2}$, and $q$ is the 4 -momentum of the virtual photon. The Bjorken $x$ is defined as

$$
x=\frac{Q^{2}}{2 P \cdot q}=\frac{Q^{2}}{2 M \nu} .
$$

Now the invariant mass of the hadrons in the final state, $W$, is expressed as

$$
W^{2}=(P+q)^{2}=M^{2}+2 P \cdot q+q^{2}=M^{2}+2 M \nu-Q^{2} .
$$

If the scattering is elastic, $W=M$. That leads $x=1$. If the scattering is inelastic, $W>M$. That leads $0<x<1$. Therefore, the Bjorken $x$ indicates the magnitude of elasticity of the scattering.

\subsubsection{Quark-Parton Model}

The Quark-Parton model provides a simple picture to understand DIS. The proton is constructed by point-like particles called partons in this model. The Quark-Parton model was suggested by Richard Feynman [6] in 1969 in order to understand high energy collisions involving hadrons. Later, it was realized that the partons can be identified with 
the quarks expected by Murray Gell-Mann [16] and George Zweig [17] in 1964 to explain the hadron spectrum.

The limit $Q^{2} \rightarrow \infty$ for a fixed value of Bjorken $x$ is known as Bjorken limit. In this limit, it can be assumed that the lepton interact with a single parton in the proton, and the transverse momenta of partons are neglected. Now we think of the 4-momentum of the parton carrying the fraction $\xi$ of the proton momentum $P$ due to the interaction with the lepton:

$$
(\xi P+q)^{2}=\xi^{2} P^{2}+2 \xi P \cdot q-Q^{2}=m^{2}
$$

where $m$ is the mass of the parton. We can now neglect the mass of the parton and nucleon at large $Q^{2}$. Then, Eq. 2.4 becomes:

$$
\xi \approx Q^{2} /(2 P \cdot q)
$$

The fraction $\xi$ is in fact the same definition as the Bjorken $x$. Therefore, the Bjorken $x$ indicates the fraction of momentum at large $Q^{2}$.

It has been experimentally observed in SLAC [18] that the structure functions, $F_{1}\left(x, Q^{2}\right)$, $F_{2}\left(x, Q^{2}\right)$, depend only on the Bjorken $x$, not on $Q^{2}$. The structure functions can be reduced as: $F_{1}\left(x, Q^{2}\right) \rightarrow F_{1}(x), F_{2}\left(x, Q^{2}\right) \rightarrow F_{2}(x)$. In the Quark-Parton model, the structure functions can be expressed as linear combinations of the probability distribution function of each flavor of quark. The distribution functions are called as the parton distribution functions (PDFs). These functions describe the underlying structure of the nucleon in terms of the probability that each parton exists with a momentum fraction between $x$ and $x+d x$.

$$
\begin{aligned}
& F_{1}(x)=\frac{1}{2} \sum_{i} e_{i}^{2} q_{i}(x) \\
& F_{2}(x)=\sum_{i} e_{i}^{2} x q_{i}(x)
\end{aligned}
$$

In these equations, $q_{i}(x)$ is the PDFs for the $i$ th quark or anti-quark flavor, and $e_{i}$ is its charge.

\subsection{Quantum Chromodynamics (QCD)}

Although the Quark Parton model well explains the structure of the nucleon, there was an inconsistency of the Pauli exclusion principle to three identical quarks in the same state in $\Delta^{++}$, for example. In order to resolve the problem, Quantum Chromodynamics (QCD) 
was developed in 1960's. It introduces "color" charge, and describes the strong interaction between the color charges. The concepts of QCD follow that of Quantum Electrodynamics (QED), which describes the interaction between charged particles. Gluons are gauge bosons of QCD such as photons are the gauge bosons of QED. In contrast to photons that have no electrical charge and cannot couple to each other, gluons carry color charge and can interact with themselves. This causes energy dependence of the strong coupling constant $\alpha_{S}$ :

$$
\alpha_{S}\left(Q^{2}\right)=\frac{12 \pi}{\left(33-2 n_{f}\right) \cdot \ln \left(Q^{2} / \Lambda^{2}\right)},
$$

where $n_{f}$ is a number of quark flavor and $\Lambda$ is a QCD scale parameter. $\Lambda$ is experimentally determined and is $\sim 250 \mathrm{MeV}$. According to the equation, when increasing $Q^{2}$, the coupling constant takes small values. In the limit $Q^{2} \rightarrow \infty$, namely in the Bjorken limit, $\alpha_{S} \rightarrow 0$, and quarks can be treated as free particles, known as asymptotic freedom.

At higher $Q^{2}$, more partons with lower fractional momenta can be observed. This means the quark distributions depends on $Q^{2}$. When $x$ is small, the structure function increases with $Q^{2}$ increases, and when $x$ is large, it decreases as $Q^{2}$ increases. That is known as violation of scaling. The parton distribution functions cannot be derived from QCD. They have to be fitted to the experimental data. However, once the parton distribution functions are determined at a certain $Q^{2}$, its $Q^{2}$ evolution can be calculated with DGLAP equation (Dokshitzer-Gribov-Liparov-Altarelli-Parisi) [20, 21, 22]:

$$
\frac{d}{d \log Q^{2}} q\left(x, Q^{2}\right)=\frac{\alpha_{s}}{2 \pi} \int_{x}^{1} \frac{d y}{y} q\left(y, Q^{2}\right) P_{q q}\left(\frac{x}{y}\right),
$$

where $\alpha_{s} P_{q q}(x / y)$ expresses the probability of a quark with a momentum fraction $y$ emitting a gluon and becomes a quark with momentum fraction $x$. The momentum fraction $x$ and $y$ are introduced in Fig. [2.2.

An example of the result of NLO (next to the leading order) global analysis [2:3] is shown in Fig. [2.3 at a scale of $Q^{2}=10 \mathrm{GeV}^{2}$ and $Q^{2}=10^{4} \mathrm{GeV}^{2}$. 


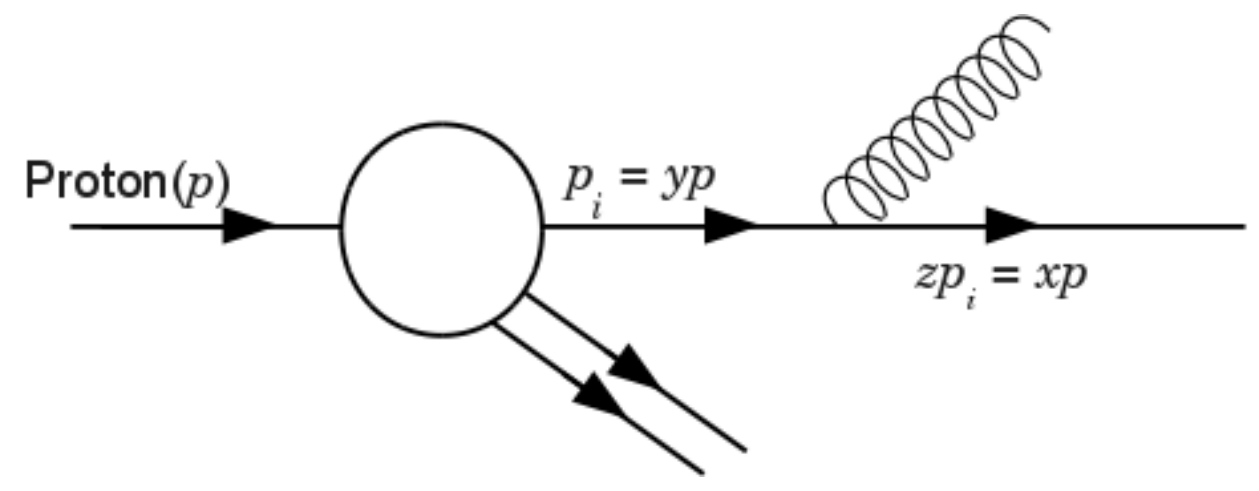

Figure 2.2: Momentum fraction for the qluon emission.

\section{MSTW 2008 NLO PDFs (68\% C.L.)}
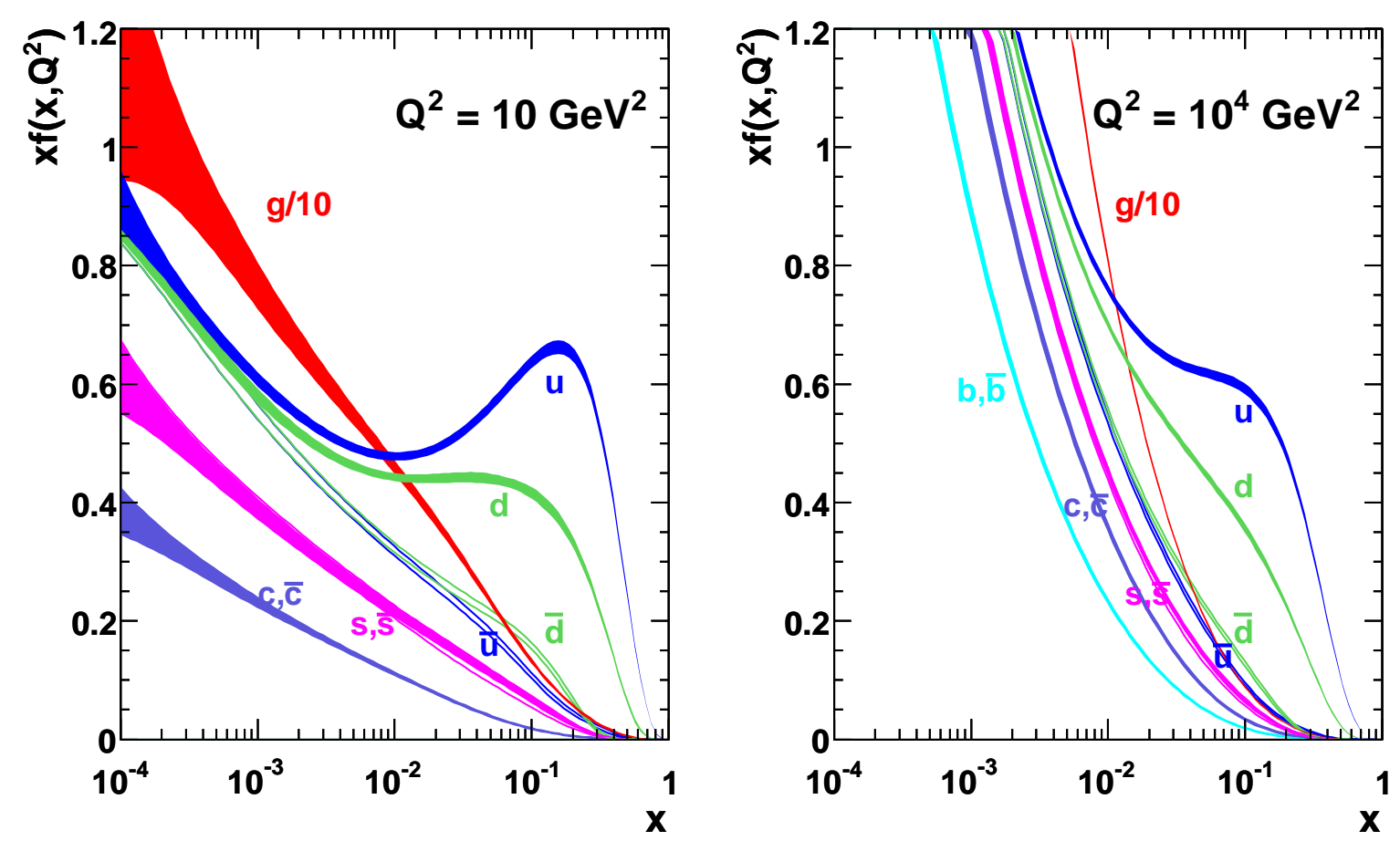

Figure 2.3: MSTW 2008 NLO PDFs for quarks and gluons inside the proton at at a scale of $Q^{2}=10 \mathrm{GeV}^{2}$ (left) and $Q^{2}=10^{4} \mathrm{GeV}^{2}$ (right) [2:3]. 


\subsection{Drell-Yan Process}

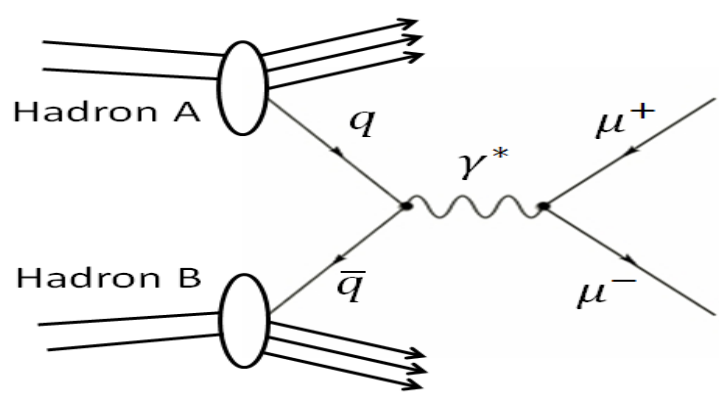

Figure 2.4: Kinematics of the Drell-Yan process. The process is a hadron A - hadron B collision in which a quark in one hadron and an anti-quark in the other hadron annihilate into a virtual photon and then a muon pair is created.

\subsubsection{Cross section for the Drell-Yan process}

The Drell-Yan process is a good tool to study anti-quarks in the proton. It takes place in hadron-hadron scattering. In the leading order of this process, a quark from one hadron and an anti-quark from the other hadron annihilate into a virtual photon, and then it decays into di-lepton as can be seen in Fig. 2.4. The virtual photon in this process is called 'time-like photon' while the photon in the DIS is called 'space-like photon'. The Drell-Yan process is well suited to measure the anti-quark distributions of the proton, since an anti-quark is always involved in this process. The cross section for the Drell-Yan process in the leading order is given by:

$$
\frac{d^{2} \sigma}{d x_{1} d x_{2}}=\frac{4 \pi \alpha^{2}}{9 M^{2}} \sum_{i} e_{i}^{2}\left[q_{i}\left(x_{1}\right) \bar{q}_{i}\left(x_{2}\right)+\bar{q}_{i}\left(x_{1}\right) q_{i}\left(x_{2}\right)\right],
$$

where $M$ is an invariant mass of the di-lepton and the sum is over all quark flavors. Here, the subscripts 1 and 2 denote a parton in the beam hadron and target hadron, respectively.

The sensitivity of the Drell-Yan process to the anti-quark distributions of the target hadron and beam hadron is obvious from Eq. 2.9. At large $x$, the parton distributions are dominated by the valence distributions and at small $x$ by the sea distributions as shown in Fig. 2.3. Thus, in the limit of large $x_{1}$ and small $x_{2}$, the cross section is dominated by the first term of Eq. [2.9. It means that the Drell-Yan process has a direct sensitivity to the anti-quark of the target nucleon in this limit. This limit is exactly the kinematics 
selected by a dipole-based spectrometer's acceptance in a fixed-target environment where all particles are boosted in forward direction.

\subsubsection{Kinematics of the Drell-Yan process}

One can measure experimentally the momenta of the lepton and antilepton from the DrellYan process. That allows us to reconstruct the virtual photon's mass, $M_{\gamma^{*}}^{2}$, longitudinal momenta, $p_{l}$, and transverse momenta, $p_{\perp}$. In the Drell-Yan experiment, it is convenient to introduce the variables:

$$
\tau=M_{\gamma^{*}}^{2} / s
$$

and the rapidity

$$
y=\frac{1}{2} \ln \left(\frac{E+p_{l}}{E-p_{l}}\right)
$$

where $s$ is the center of mass energy squared of the interacting hadrons and $E$ is the energy of the virtual photon. From these variables, the momentum fractions $x_{1}$ and $x_{2}$ of the interacting partons are given by:

$$
x_{1,2}=\left(\tau+\frac{p_{\perp}^{2}}{s}\right)^{1 / 2} e^{ \pm y}
$$

and Feynman- $x$ is then defined as follows

$$
x_{F} \equiv \frac{2 p_{l}}{\sqrt{s}} \approx x_{1}-x_{2}
$$




\section{Chapter 3}

\section{Review of Experiments on Flavor Asymmetry of Anti-quark Sea}

One of the physics motivations of the SeaQuest experiment is to determine the asymmetry between $\bar{d}$ and $\bar{u}$ in the proton as a function of Bjorken $x$. The history of the investigation of the asymmetry of anti-quark distributions and the methods are described in this chapter.

\subsection{NMC experiment}

The asymmetry of anti-quark distributions in the proton is observed by the New Muon Collaboration (NMC) $[8,9]$ for the first time. They tested the Gottfried sum rule by measuring the cross section ratio for deep inelastic scattering of muons from hydrogen and deuterium. The muon beam energies used were 90 and $280 \mathrm{GeV}$. The Gottfried sum $S_{g}$ is defined as

$$
S_{g} \equiv \int_{0}^{1}\left[F_{2}^{p}(x)-F_{2}^{n}(x)\right] \frac{d x}{x},
$$

where $F_{2}$ is expressed in Eq. 2.6. The Gottfried sum $S_{g}$ is

$$
S_{g}=\int_{0}^{1} \sum_{i} e_{i}^{2}\left[q_{i}^{p}(x)+\bar{q}_{i}^{p}(x)-q_{i}^{n}(x)-\bar{q}_{i}^{n}(x)\right] d x
$$

in the Quark-Parton model, where $e_{i}$ is the charge (in unit of $e$ ) of a quark of flavor $i$. Under the assumption of isospin symmetry between the proton and the neutron, Eq. उ.2 reduces to

$$
S_{g}=\int_{0}^{1} \frac{1}{3}[u(x)+\bar{u}(x)-d(x)-\bar{d}(x)] d x,
$$


where $u(x) \equiv u^{p}(x)=d^{n}(x), \bar{u}(x) \equiv \bar{u}^{p}(x)=\bar{d}^{n}(x), d(x) \equiv d^{p}(x)=u^{n}(x)$ and $\bar{d}(x) \equiv$ $\bar{d}^{p}(x)=\bar{u}^{n}(x)$. Eq. B.3 can be rewritten as

$$
S_{g}=\int_{0}^{1} \frac{1}{3}[u(x)-\bar{u}(x)] d x-\int_{0}^{1} \frac{1}{3}[d(x)-\bar{d}(x)] d x+\int_{0}^{1} \frac{2}{3}[\bar{u}(x)-\bar{d}(x)] d x .
$$

The first two integrals can be calculated using the definition of the valence quarks: two up quarks and one down quark in the proton. Eq. 3.4 therefore reduces to

$$
S_{g}=\frac{1}{3}+\int_{0}^{1} \frac{2}{3}[\bar{u}(x)-\bar{d}(x)] d x .
$$

If $\int_{0}^{1} \bar{u}(x) d x=\int_{0}^{1} \bar{d}(x) d x$, then Eq. 3.5 arrives at the traditional expectation of $\frac{1}{3}$ (the Gottfried sum rule). The NMC measurement obtained the Gottfried sum[9],

$$
S_{g}=0.235 \pm 0.026
$$

This value is lower than $\frac{1}{3}$. It indicates that $\bar{d}(x)$ is larger than that of $\bar{u}(x)$. The result of the NMC measurement was the first indication of inequality of the anti-down $\left(\bar{d}_{p}\right)$ and anti-up $\left(\bar{u}_{p}\right)$ quark distributions in the proton. It suggests that the proton has more $\bar{d}_{p}$ than $\bar{u}_{p}$.

\section{$3.2 \quad$ NA51 experiment}

The NA51 experiment[10] used the $450 \mathrm{GeV} / c(\sqrt{s}=29 \mathrm{GeV})$ proton beam from the CERN Synchrotoron Proton Source and the NA10 spectrometer in order to compare the Drell-Yan dimuon yields from hydrogen and deuterium targets. The NA51 experiment is the first experiment that measures the ratio of $\bar{d}(x) / \bar{u}(x)$ in the proton. The ratio is measured by comparing the cross section of the Drell-Yan process in the proton-proton interaction, $\sigma^{p p}$, and the proton-deuteron interaction, $\sigma^{p d}$. Those cross sections are given by:

$$
\begin{aligned}
\sigma^{p p} & \propto \frac{4}{9} u\left(x_{1}\right) \bar{u}\left(x_{2}\right)+\frac{1}{9} d\left(x_{1}\right) \bar{d}\left(x_{2}\right) \\
\sigma^{p n} & \propto \frac{4}{9} u\left(x_{1}\right) \bar{d}\left(x_{2}\right)+\frac{1}{9} d\left(x_{1}\right) \bar{u}\left(x_{2}\right),
\end{aligned}
$$

where the heavier quark terms have been ignored and isospin symmetry was used to equate $\bar{d}_{p}(x)$ to $\bar{u}_{n}(x)$ and $\bar{u}_{p}(x)$ to $\left.\bar{d}_{n} \overline{(} x\right)$. The cross section of the Drell-Yan process in the proton-deuteron interaction can be assumed as

$$
\sigma^{p d}=\sigma^{p p}+\sigma^{p n}
$$


where the nuclear effects inside the deuteron are ignored. Therefore, the ratio of the cross section can be written as

$$
\begin{aligned}
\frac{\sigma^{p d}}{2 \sigma^{p p}} & =\frac{1}{2} \frac{\sigma^{p p}+\sigma^{p n}}{\sigma^{p p}} \\
& =\frac{1}{2}\left[\frac{\frac{4}{9} u\left(x_{1}\right) \bar{u}\left(x_{2}\right)+\frac{1}{9} d\left(x_{1}\right) \bar{d}\left(x_{2}\right)+\frac{4}{9} u\left(x_{1}\right) \bar{d}\left(x_{2}\right)+\frac{1}{9} d\left(x_{1}\right) \bar{u}\left(x_{2}\right)}{\frac{4}{9} u\left(x_{1}\right) \bar{u}\left(x_{2}\right)+\frac{1}{9} d\left(x_{1}\right) \bar{d}\left(x_{2}\right)}\right]
\end{aligned}
$$

If the numerator and denominator of Eq. 3.10 are divided by $\frac{4}{9} u\left(x_{1}\right) \bar{u}\left(x_{2}\right)$, and the formula reduces to

$$
\frac{1}{2}\left[\frac{1+\frac{1}{4} \frac{d\left(x_{1}\right) \bar{d}\left(x_{2}\right)}{u\left(x_{1}\right) \bar{u}\left(x_{2}\right)}+\frac{\bar{d}\left(x_{2}\right)}{\bar{u}\left(x_{2}\right)}+\frac{1}{4} \frac{d\left(x_{1}\right)}{u\left(x_{1}\right)}}{1+\frac{1}{4} \frac{d\left(x_{1}\right) \bar{d}\left(x_{2}\right)}{u\left(x_{1}\right) \bar{u}\left(x_{2}\right)}}\right]
$$

Eq. B.Tl can be factorized as

$$
\frac{1}{2}\left[\frac{1+\frac{d\left(x_{1}\right)}{4 u\left(x_{1}\right)}}{1+\frac{d\left(x_{1}\right)}{4 u\left(x_{1}\right)} \frac{\bar{d}\left(x_{2}\right)}{\bar{u}\left(x_{2}\right)}}\right]\left[1+\frac{\bar{d}\left(x_{2}\right)}{\bar{u}\left(x_{2}\right)}\right] .
$$

In the limit of $x_{1} \gg x_{2}, d(x) \ll 4 u(x)$ can be assumed, and then, Eq. B. 2 even further reduces to

$$
\left.\frac{\sigma^{p d}}{2 \sigma^{p p}}\right|_{x_{1} \gg x_{2}} \simeq \frac{1}{2}\left[1+\frac{\bar{d}\left(x_{2}\right)}{\bar{u}\left(x_{2}\right)}\right] .
$$

Eq. 3.13 is obtained with following assumptions:

- The next-leading order (NLO) term of the Drell-Yan process is not considered.

- The formula only considers quark from the beam proton and anti-quark from the target proton.

- The formula assumes that $d(x)$ is much smaller than $4 u(x)$.

The NA51 experiment concluded

$$
\frac{\bar{u}(x)}{\bar{d}(x)}=0.51 \pm 0.04 \text { (stat.) } \pm 0.04 \text { (sys.) }
$$

where the Bjorken $x$ of the parton from the target proton is 0.18 . This is a clear indication of the asymmetry of the sea anti-quark distributions. However, this result cannot conclude the asymmetry of the integration of $\bar{d}$ and $\bar{u}$ in the proton that the NMC experiment measured because of the Bjorken $x$ limitation in the measurement. 


\section{3 $\quad$ E866/NuSea experiment}

The E866/NuSea[[12, 13, 14] experiment at Fermi National Accelerator Laboratory (Fermilab) was a fixed-target Drell-Yan experiment. This experiment measured the ratio of $\bar{d}(x) / \bar{u}(x)$ in the proton at $0.015<x_{2}<0.35$ while the NA51 experiment measured it only at $x_{2}=0.18$. The same method as the NA51 experiment is used. The E866/NuSea experiment used the $800 \mathrm{GeV} / c$ proton beam extracted from the Fermilab Tevatron accelerator. Hydrogen and deuterium targets were used. Three large dipole magnets provide the momentum analysis of muons. The magnetic fields are in the horizontal direction, bending the tracks in the vertical direction. The polarities and currents of the magnets were adjusted to select particular ranges of dimuon mass, while minimizing the background in the wire detectors. The position of each muon was measured precisely at three groups of wire detectors and hodoscopes.

The data were taken with three mass settings of the spectrometer magnets; the high, intermediate, and low mass settings. They recorded $\sim 360,000$ Drell-Yan events in total, approximately two thirds of them from a deuterium target and the rest from a hydrogen target. The ratio of Drell-Yan cross sections, $\sigma^{p d} / 2 \sigma^{p p}$, and the extracted ratio of $\bar{d}(x) / \bar{u}(x)$ are shown in Fig. $[.3$ in blue. The E866/NuSea data shows an interesting result of the distribution of sea quark in the proton. The ratio $\bar{d}(x) / \bar{u}(x)$ is as large as 1.7 at $x_{2} \sim 0.2$, and seems to be less than one at $x_{2} \sim 0.3$. Several theoretical models of the proton structure were proposed to reproduce this behavior [57, 58, 59]. However, none of them succeeded in reproducing the result at higher Bjorken $x(x>0.3)$ where the ratio becomes smaller than one. This may shed some light on the origin of the sea quarks.

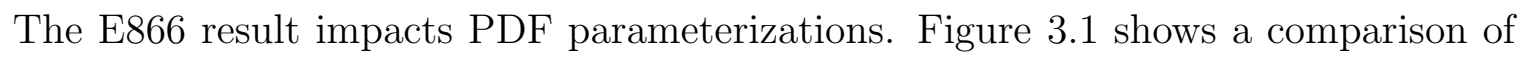
$\bar{d} / \bar{u}$ distribution in CTEQ4M[24] and CTEQ5M[25]. The major change is due to the E866 data. 


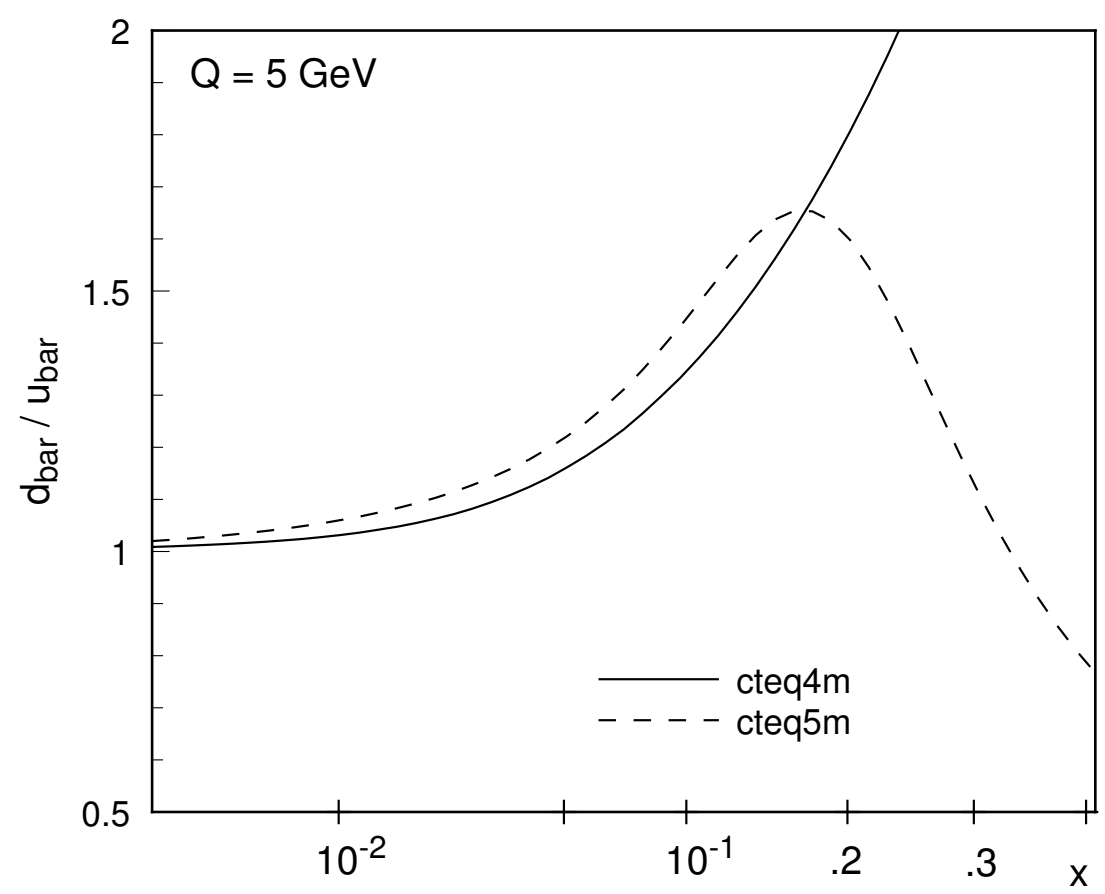

Figure 3.1: Comparison of $\bar{d} / \bar{u}$ distribution in CTEQ4M and CTEQ5M. The major change is due to the E866 data[25] 


\subsection{Motivation for the SeaQuest Experiment}

E906/SeaQuest experiment at Fermilab was planned following the E866/NuSea experiment. As described in the previous subsection, the behavior of the flavor asymmetry at high Bjorken $x$ is very curious. The SeaQuest experiment measures the asymmetry at $0.1<x<0.45$ as shown in Fig. 1.3 in red. The basic idea of the SeaQuest spectrometer follows the E866 settings, but it focuses on higher $x$ region.

SeaQuest uses the $120 \mathrm{GeV}$ proton beam from Fermilab Main Injector $(\sqrt{s} \sim 15 \mathrm{GeV}$ ) for measuring the ratio of $\bar{d}(x) / \bar{u}(x)$ at a high Bjorken $x$, while the E866 experiment used the $800 \mathrm{GeV}$ proton beam from Tevatron at Fermilab. Here are two advantages in using the beam at lower energy.

First, for fixed Bjorken $x_{1}$ and $x_{2}$, the Drell-Yan cross section is proportional to $1 / E_{b}$ where $E_{b}$ is the incident beam. The $120 \mathrm{GeV}$ Main Injector experiment will have a seven times larger cross section compared with the previous experiment which used the $800 \mathrm{GeV}$ beam.

Second, practical limitation in the acceptable luminosity for these experiments is the single muon rates in the detectors. In E866 experiment at $800 \mathrm{GeV}, J / \psi$ events from the beam dump were a significant contribution to the muon singles rates. The $J / \psi$ production rate at $120 \mathrm{GeV}$ is roughly seven times smaller when compared with $800 \mathrm{GeV}$. The combination of these two effects is expected to increase the number of recorded events by a factor of 50 at high $x$ in comparison with the previous Drell-Yan experiment.

\subsection{Calculation of kinematics}

SeaQuest measures the momentum of di-muon from the Drell-Yan process, then obtain 4-momenta of $p_{\mu^{+}}$and $p_{\mu^{-}}$. That allows us to reconstruct the invariant mass of the dimuon. The momentum of the virtual photon is evaluated by just summing up the momenta of $\mu^{+}$and $\mu^{+}: p_{\gamma^{*}}=p_{\mu^{+}}+p_{\mu^{-}}$. The momentum in the center-of-mass frame of colliding hadrons is evaluated by Lorentz boost. The rapidity is calculated by Eq. $2 . \Pi$, and then $x_{1}$ and $x_{2}$ are given by Eq. 2.22 using the 4-momentum in the frame.

\subsection{Future $d / \bar{u}$ experiments}

There is a plan to extend the $d \overline{/} \bar{u}$ measurement to much larger values of Bjorken- $x$ using the $50-\mathrm{GeV}$ Proton Synchrotron (PS) at J-PARC[6(1)]. Such information is crucial for 


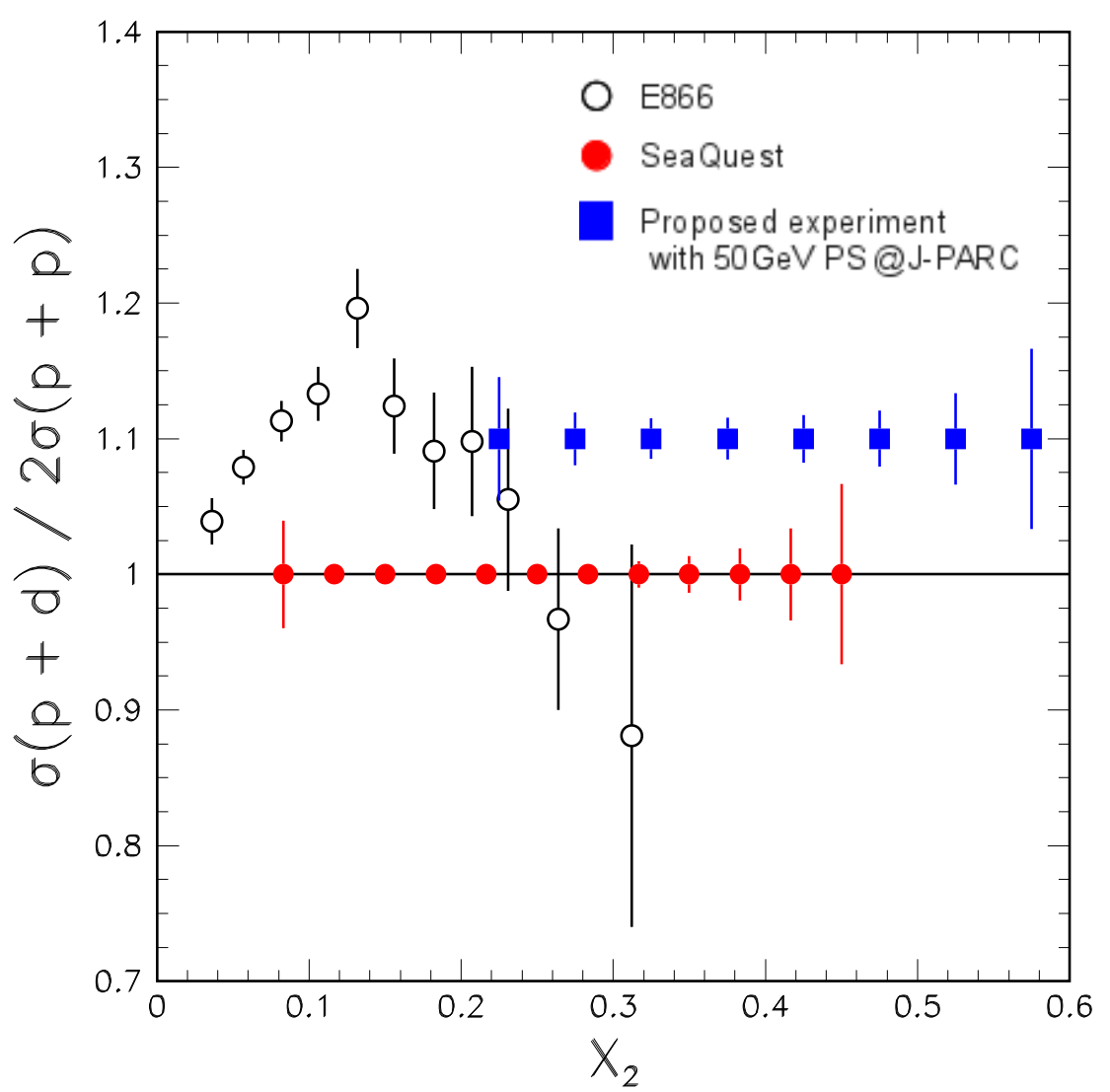

Figure 3.2: Open circles show Cross section ratio of $\sigma^{p d} / 2 \sigma^{p p}$ from E866. Red bars indicate expected statistical uncertainty of SeaQuest. Blue bars indicate expected statistical uncertainty of the proposed experiment at the $50 \mathrm{GeV}$ PS. The positions of red and blue are arbitrary chosen.

understanding the origins of flavor asymmetry in the sea of the proton. As mentioned in Sec. [..4, for given values of $x_{1}$ and $x_{2}$ the Drell-Yan cross section is proportional to $1 / \mathrm{s}$. Thus, the Drell-Yan cross section at $50 \mathrm{GeV}$ is roughly 2.4 times larger than at $120 \mathrm{GeV}$. Figure 3.2 shows the expected statistical accuracy for cross section ratio of $\sigma^{p d} / 2 \sigma^{p p}$ at the $50 \mathrm{GeV}$ PS $(\sqrt{s} \sim 9.9 \mathrm{GeV})$ compared with the data from E866 and SeaQuest. A definitive measurement of the $d \overline{/} \bar{u}$ over the region $0.25<x<0.7$ could be obtained at the $50 \mathrm{GeV}$ PS. 


\section{Chapter 4}

\section{Experimental Method: SeaQuest}

The SeaQuest experiment is a fixed target experiment performed at Fermi National Accelerator laboratory (Fermilab). It uses the $120 \mathrm{GeV}$ proton beam extracted from Fermilab Main Injector as shown in Fig. 4.]. The Main Injector is shown at the upper left in the figure. The extracted proton beam is switched at the switchyard and sent to the experimental hall of SeaQuest.

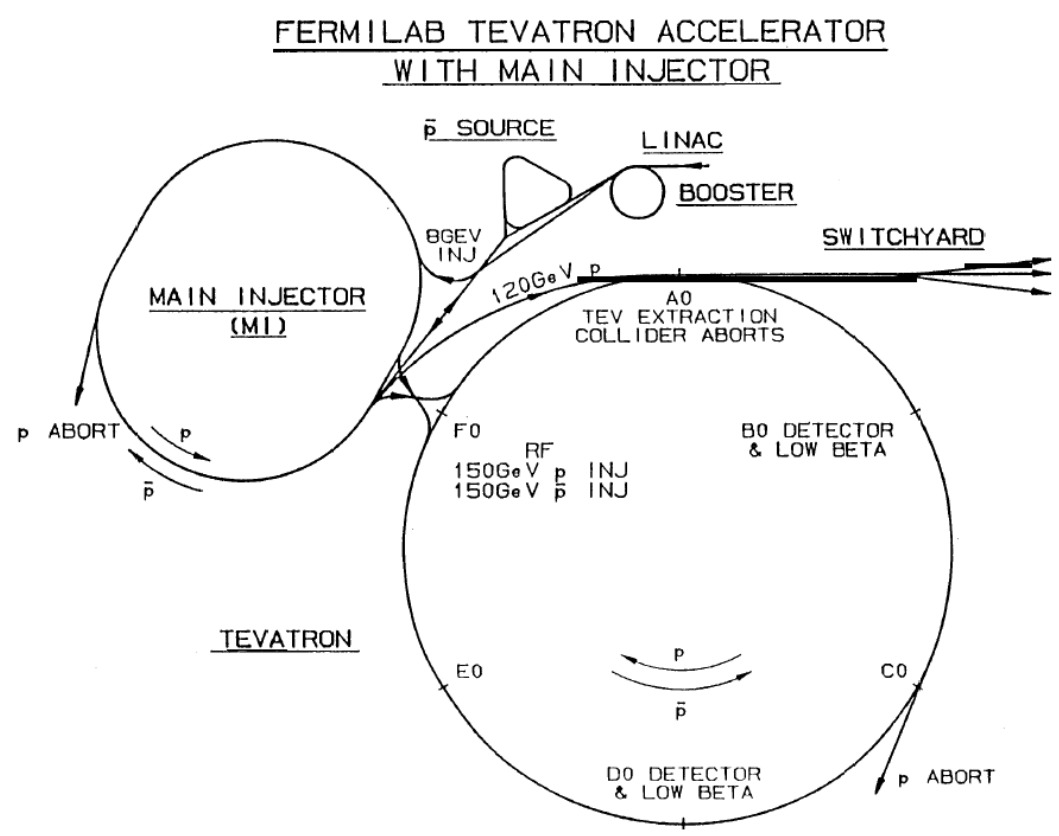

Figure 4.1: SeaQuest uses the $120 \mathrm{GeV}$ proton beam extracted from Fermilab Main Injector [26] (upper left).

The SeaQuest experiment is designed and optimized for the $120 \mathrm{GeV}$ proton beam. The earlier experiment E866/NuSea used the $800 \mathrm{GeV}$ proton beam. The construction 
of the SeaQuest spectrometer started in 2009. The first data using the proton beam was taken in 2012. The purpose of this run was mainly detector commissioning. Detector upgrade has been done after the commissioning run for one and half year in parallel to the accelerator upgrade. Physics data taking started in 2013. The detail of SeaQuest history is listed in Table 4.]. This thesis shows the result of the analysis using the data taken in the Run II and Run III.

\begin{tabular}{ll}
\hline \hline Term & Run \\
\hline $2009.03-2012.03$ & Experimental construction \\
$2012.03-2012.05$ & Run I (Detector commissioning run) \\
$2012.05-2013.11$ & Detector upgrade \\
$2013.11-2014.09$ & Run II (Physics run) \\
$2014.09-2014.11$ & Accelerator shutdown \\
$2014.11-2015.08$ & Run III (Physics run) \\
$2015.08-2015.11$ & Accelerator shutdown \\
$2015.11-$ & Run IV (Physics run) \\
\hline
\end{tabular}

Table 4.1: History of the SeaQuest experiment. This thesis shows the results of analysis using the data taken in the Run II and Run III.

All equipments of the SeaQuest spectrometer are upgraded and optimized to measure $\bar{d} / \bar{u}$ at wider $x$ range than the E866 experiment. The proton beam interacts with SeaQuest target located upstream of the spectrometer. The SeaQuest spectrometer detects opposite charge muon pairs produced in proton-nucleon interaction. The overview of the spectrometer is shown in Fig. 4.2. It has two dipole magnets, one to focus the high momentum muons and defocus low momentum muons, called "FMAG", and the other to measure the muon momenta, called "KMAG". There are four groups of tracking detectors called "Stations". Each tracking Station is named with a number like "Station 1" for the first Station. Stations 1, 2, and 3 are composed of scintillator hodoscope planes and drift chamber(s). Only Station 3 has two drift chambers in order to cover the large detection area while the other tracking stations have one drift chamber. The scintillator hodoscope planes provide hit information to the trigger system. The hit information from the drift chambers is used to obtain track information of the muons. Station 4 is composed of the scintillator hodoscope planes for triggering like other Stations. However, drift tubes are used for muon tracking instead of drift chambers. Only muons can penetrate into the tracking Stations because of a beam dump placed in FMAG to stop hadrons. There is a Zinc and concrete wall between Station 3 and Station 4 to ensure that no hadrons penetrate into Station 4. Therefore, Station 4 also serves as a muon identifier. Those 


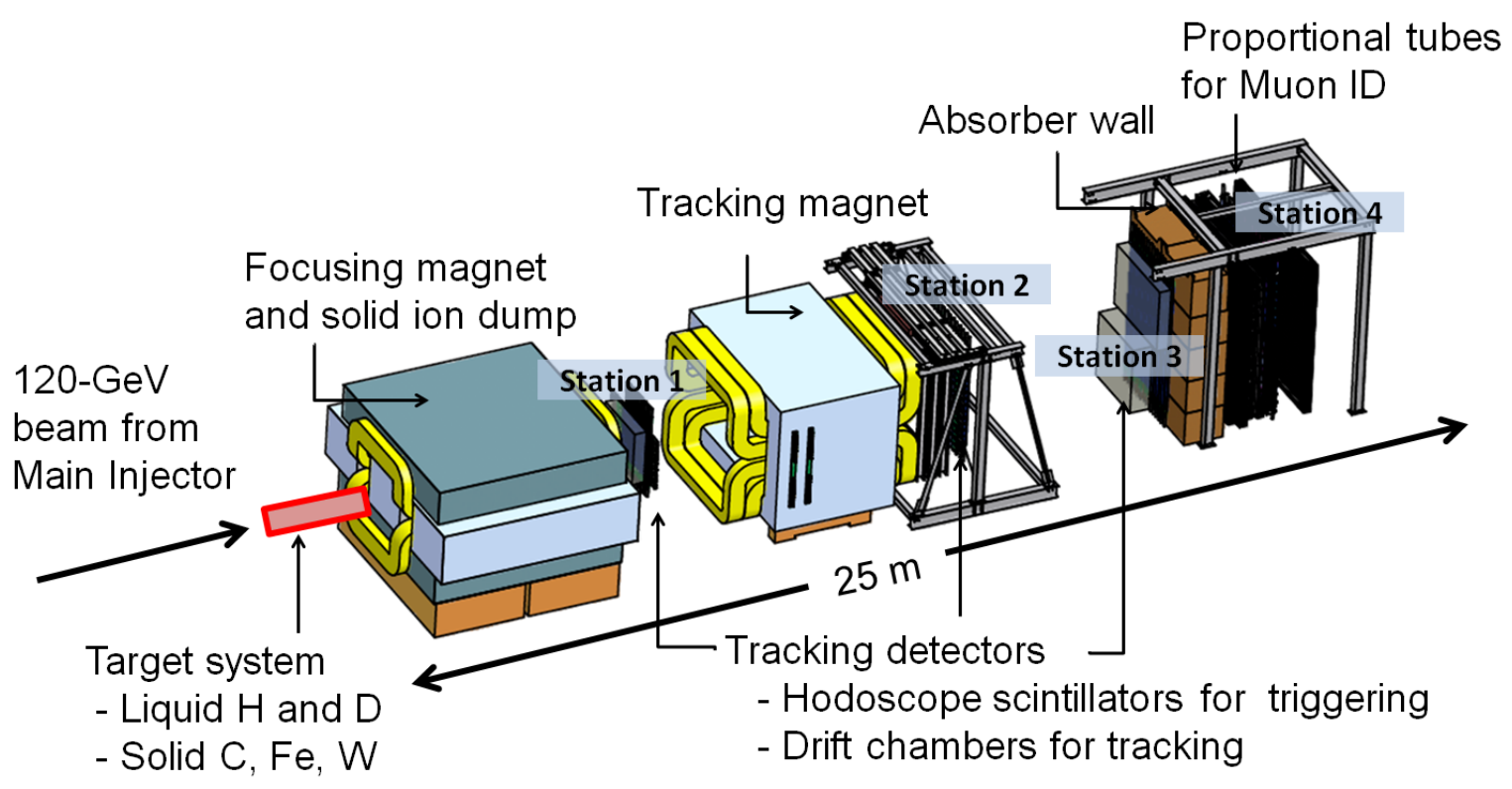

Figure 4.2: Overview of the SeaQuest spectrometer. This spectrometer has four tracking stations for detecting charged particles. A $120 \mathrm{GeV}$ proton beam comes from left side of the figure, and then collides with the SeaQuest target located upstream of the spectrometer. There are four groups of tracking detectors called "Station". Each Station has scintillator hodoscope planes for triggering. Station 1-3 have drift chamber(s) while Station 4 has drift tubes for tracking. There are two dipole magnets: focusing magnet (FMAG) and tracking magnet (KMAG). There are two hadron absorbers: one is placed in FMAG and the other one is placed between Station 3 and Station 4. The length of this spectrometer from the targets to the end is about $25 \mathrm{~m}$. 
equipments are described in detail in the next subsections.

The $x-y-z$ coordinate system adopted in the experiment has the positive $z$-axis along the incoming beam direction, the $y$-axis oriented vertically upwards, and the $x$-axis is chosen to complete a right-handed coordinate system. The origin of $z$-axis, $z=0$, is placed at the upstream surface of the first magnet, FMAG. 


\subsection{Accelerator and Beam}

The time structure of the proton beam is shown in Fig. 4.3. The Main Injector provides proton beam for a 5 second once in every minute to the SeaQuest experimental hall. The 5 -second-long beam is called a "spill". In the remaining 55 seconds, the beam is sent to other fixed-target experiments. The Main Injector is operated at a $53 \mathrm{MHz}$ radiofrequency $(\mathrm{RF})$, and protons are grouped in each RF "bucket". The Booster provides a "train" containing $83 \mathrm{RF}$ buckets filled with $8 \mathrm{GeV}$ protons to the Main Injector. The Main Injector can hold up to seven trains. But six out of the seven trains are typically filled with protons, not all the trains, in order to inject protons from the Booster without disturbing protons already filled in the Main Injector. Therefore, the Main Injector typically holds $498 \mathrm{RF}$ buckets in total in its ring. Since the Booster cycles at $15 \mathrm{~Hz}, 0.4$ seconds are required to fill the Main Injector. Then, the proton beam is accelerated to $120 \mathrm{GeV}$ in a single turn. After reaching the maximum energy $(120 \mathrm{GeV})$, part of protons in each RF bucket are cut off by slow extraction system and sent to the experimental hall. The length of each bucket is 1-2 ns. Each bucket arrives at the experimental hall every $\sim 18.9 \mathrm{~ns}$ during the spill due to the $53 \mathrm{MHz}$ of RF frequency. The time structure of the proton beam is shown in Fig. 4.3. The designed number of protons in one RF bucket that arrives at the target is roughly $4 \times 10^{4}$. The typical intensity (number of protons) received in one spill is between $1 \times 10^{12}$ and $1 \times 10^{13}$ protons. 


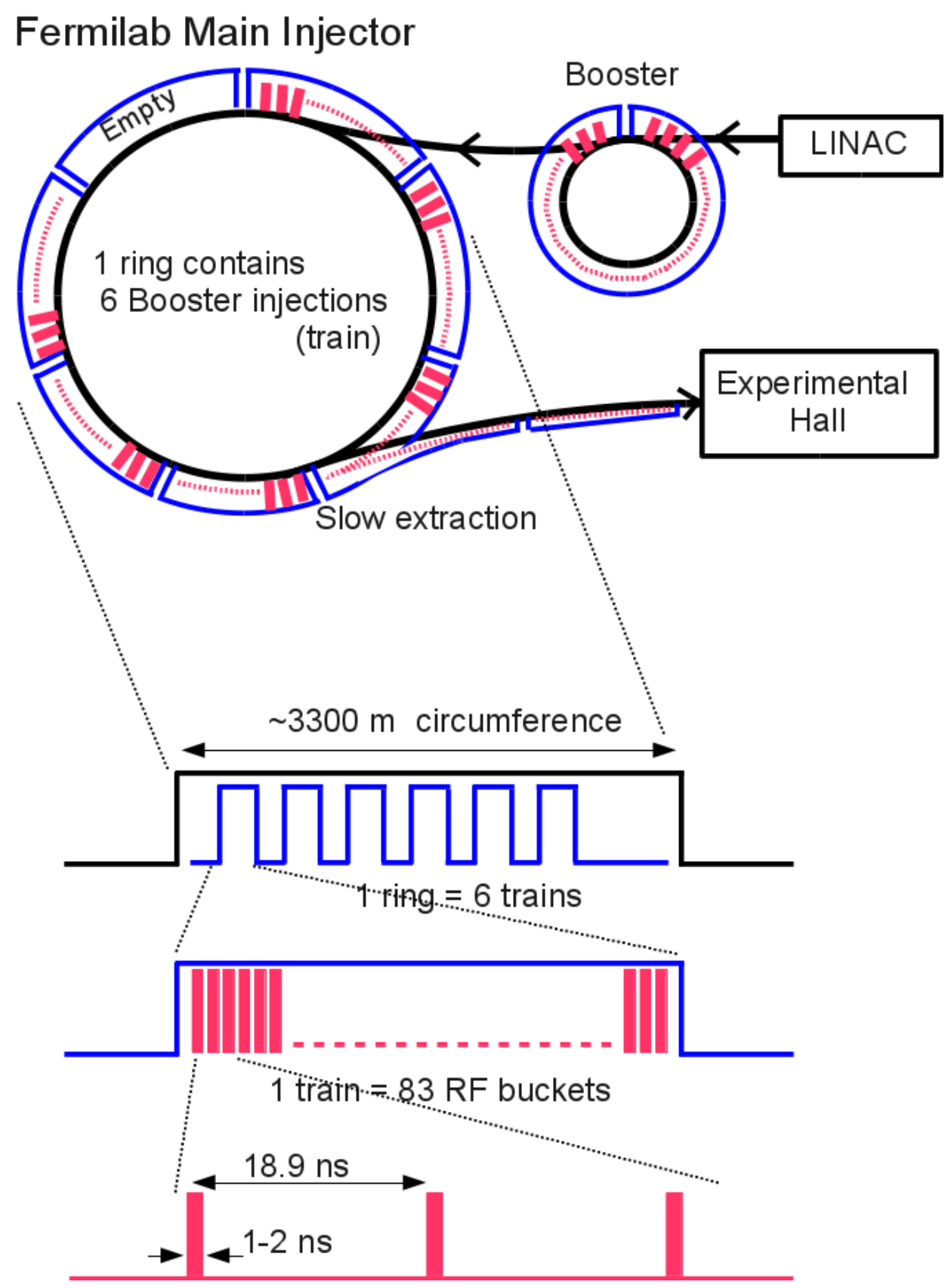

Figure 4.3: The beam structure of the Main Injector proton beam. Protons are accelerated to $8 \mathrm{GeV}$ at the Booster. The protons are then injected from the Booster to the Main Injector. The Main Injector typically holds six Booster injections (trains), and each train holds $83 \mathrm{RF}$ buckets. The protons are accelerated to $120 \mathrm{GeV}$ in the Main Injector, and then sent to the experimental hall by the slow extraction system. The protons arrive in 1-2 ns-long RF bucket spaced by 18.9 ns at the SeaQuest experimental hall. 


\subsection{Beam Monitor}

There are several beamline detectors in order to record and monitor the size, position and intensity of the proton beam. The beam intensity is monitored by an ion chamber (NM3ION), secondary emission monitor (G2SEM), and Cherenkov counter. Those monitors are located upstream of the SeaQuest target. Both the names, "NM3" and "G2", of the monitors come from the names of the location of these monitors in the beam line. In addition, the size and the position of the beam are monitored by segmented wire ion chambers (SWICs) located in front of the SeaQuest target. Both NM3ION and G2SEM help us to know the absolute value of the intensity received in each spill. But, it was required to monitor also the number of protons of each RF bucket, not only the number of protons of each spill in order to do RF bucket level analysis:

- Monitor the total number of protons received when our DAQ is ready to record data

- Cut the RF bucket that has anomalously larger number of protons that produce lots of secondary particles and is difficult to analyze.

The Cherenkov counter and its readout electronics are designed to have a good time resolution for this purpose.

A drawing of the Cherenkov counter is shown in Fig. 4.4. It is filled with a gas mixture of $80 \%$ Argon and $20 \% \mathrm{CO}_{2}$. The pressure of the gas in the counter is equal to the atmospheric pressure. The momentum threshold for the proton beam is $\sim 37 \mathrm{GeV} / \mathrm{c}$ as the index of refraction of Argon $/ \mathrm{CO}_{2}$ is 1.000315 [1.9]. A 45 degree mirror of aluminized Kapton held on an elliptical G10 frame directs the Cherenkov light to a single photomultiplier tube. A baffle of black construction paper held parallel to the mirror ensures that the proton beam path length in the gas mixture is independent of the beam position. A two-inch diameter photomultiplier tube is positioned close to the mirror so that all Cherenkov light falls directly on the face of the phototube. Its readout system is called QIE (charge $(\mathrm{Q})$ integrator encoder) board programmed by $\mathrm{C}++$ and ROOT [27] in order to encode the amount of charge accumulated. It is synchronized with Main Injector $\mathrm{RF}$ clock for the correct record of the bucket-by-bucket intensity. Its beam intensity is normalized using the G2SEM result, since the counter monitors the relative intensity by measuring the intensity of the Cherenkov light. 


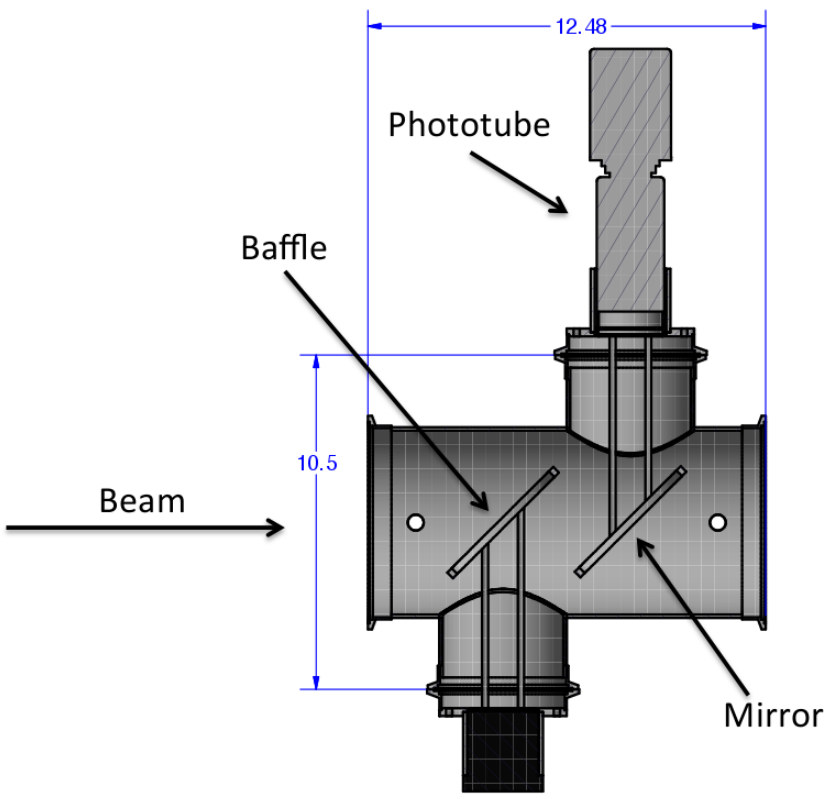

Figure 4.4: Drawing of the Cherenkov beam counter. The size is written in inches. A 45 degree mirror of aluminized Kapton held on an elliptical G10 frame directs the Cherenkov light to a single photomultiplier tube. A baffle of black construction paper held parallel to the mirror ensures that the proton beam path length in the gas mixture is independent of the beam position. A two-inch diameter photomultiplier tube is positioned close to the mirror so that all Cherenkov light falls directly on the face of the phototube. 


\subsection{Target}

The SeaQuest target system has seven targets in total: liquid hydrogen (LH2), liquid deuterium (LD2), iron, carbon, tungsten, empty target flask, and empty position (nothing is set at this position). LH2 and LD2 targets are enclosed in an insulated vacuum jacket that has thin windows at both ends of the target flask, respectively. The side view of the flask is shown in Fig. 4.5. The length of the LH2 and LD2 target is 20 inch which is equivalent to $\sim 7 \%$ (LH2) or $\sim 15 \%$ (LD2) of the nuclear interaction length. One empty target flask and one empty position are used to count background events to be subtracted from other target rates. Three nuclear targets, iron, carbon, and tungsten, are used for nuclear dependence studies. The length of those targets is equivalent to $\sim 15 \%$ of a nuclear interaction length.

There are windows and flask heads on the both sides of the target flask (LH2, LD2 and empty target) on the beam axis. The window is made from titanium alloy, and its thickness is 0.0055 inch. The flask head is made from steel, and its thickness is 0.002 inch $[28]$.

The targets are located on a movable table such that they can be interchanged between spills of the beam in order to minimize systematic errors due to a long-term change in the target and beam conditions. Diagram of the table is shown in Fig. 4.6. The targets are controlled remotely to change during spills. It takes roughly 30 seconds to interchange the targets. They can be interchanged every spill, since the interval of spills is 55 seconds. If the interchange takes more than 45 seconds with some software or hardware troubles, the proton beam is stopped in the interval in the spills.

Characteristics of those targets are summarized in Table 4.2. Also, typical number of spills per cycle assigned for the targets are written. Those numbers can vary in response to sample needs and running configuration.

\begin{tabular}{llll}
\hline \hline Position & Material & Thickness & Spills/Cycle \\
\hline 1 & LH2 & $20 "$ & 10 \\
2 & Empty flask & - & 2 \\
3 & LD2 & $20 "$ & 5 \\
4 & No Target & - & 2 \\
5 & Iron & $0.75 "$ & 1 \\
6 & Carbon & $1.308 "$ & 2 \\
7 & Tungsten & $0.375 "$ & 1 \\
\hline
\end{tabular}

Table 4.2: Characteristics and typical number of spills per cycle of the SeaQuest targets. In this examples, 23 spills constitute one cycle. 


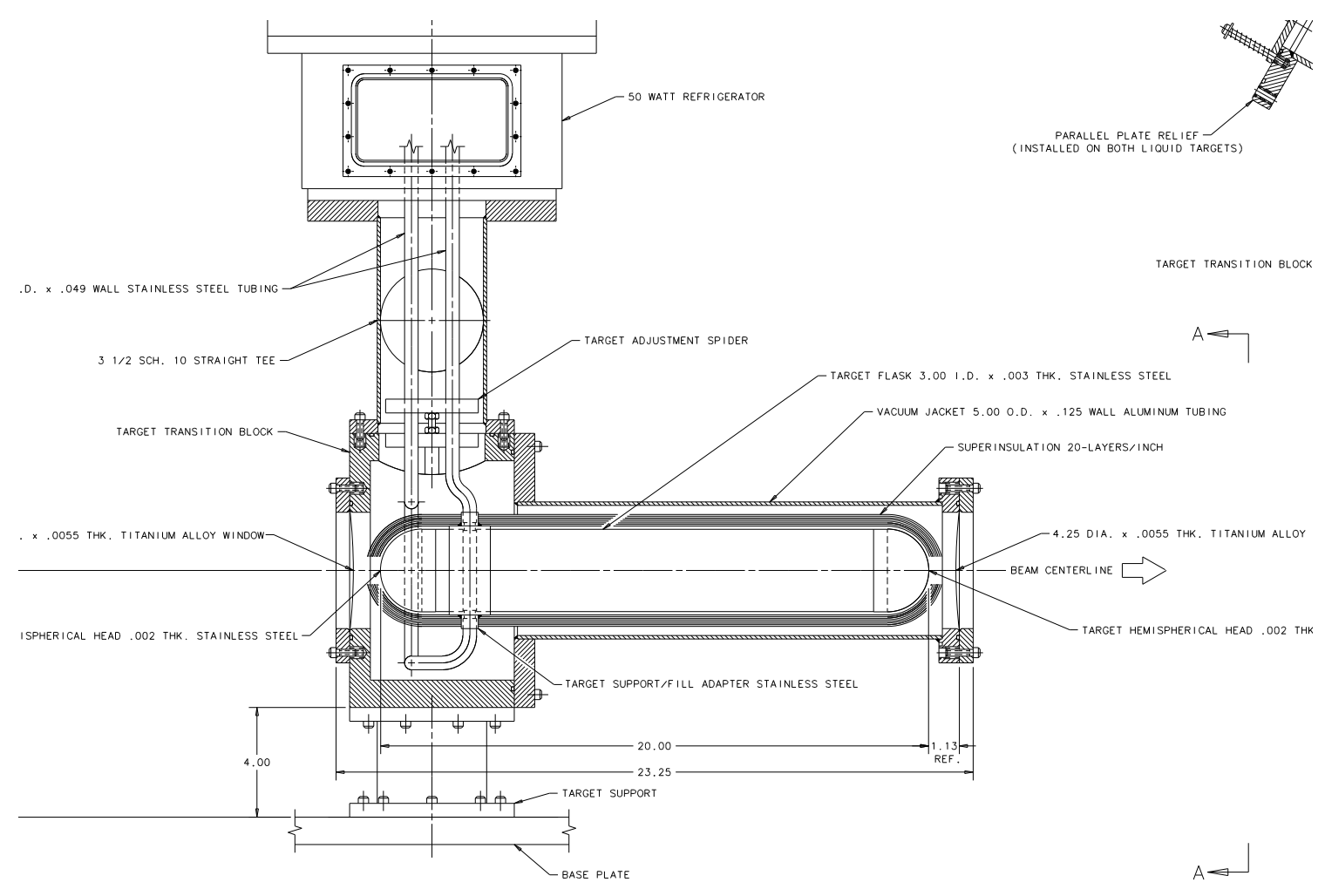

Figure 4.5: Side view of the SeaQuest target flask. The size is written in inches. Each target is enclosed in an insulated vacuum jacket that has thin windows at both ends. 


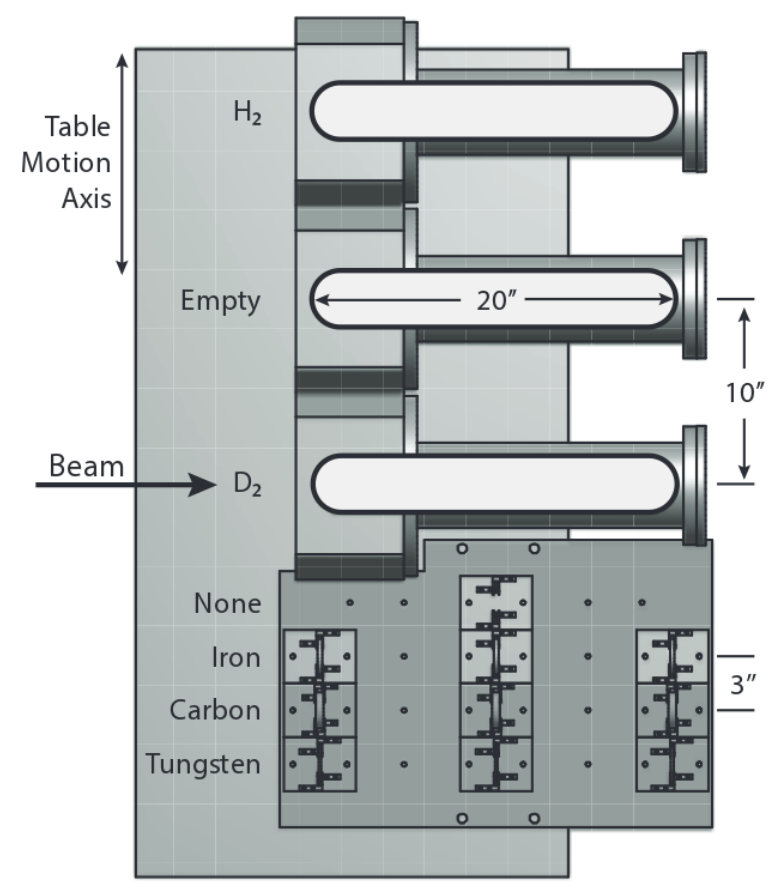

Figure 4.6: Top view of the layout of the positionable target table showing the seven target positions. The size is written in inches. 


\subsection{Magnet}

The SeaQuest spectrometer has two dipole magnets. The first magnet is called "FMAG". It is located between the target and Station 1. The geometrical dimensions of FMAG are shown in Fig. 4.7. This magnet focuses the high momentum muons into the acceptance of the spectrometer and bends low momentum muons out of the acceptance. There is a beam dump made with iron inside FMAG. The primary proton beam as well as secondary hadrons stops in the dump.

The second magnet called "KMAG" is located between Station 1 and Station 2. The geometrical dimensions of KMAG are shown in Fig. 4.8. It is used to measure the momenta of muons.

The both magnets produce a magnetic field oriented in the $+y$ direction (vertical upward). Therefore, they bend positive (negative) muons to $-(+) x$ direction (horizontal direction) as shown in Fig. 4.9. A summary of the design values of the magnets is found in Table. 4.3.

\begin{tabular}{lcc}
\hline \hline Property & FMAG & KMAG \\
\hline Length & $189 "$ & $211^{\prime \prime}$ \\
Width & $95 "$ & $147 "$ \\
Height & $198^{\prime \prime}$ & $198^{\prime \prime}$ \\
Horizontal Aperture & $48^{\prime \prime}(123 \mathrm{~cm})$ & $63^{\prime \prime}(160 \mathrm{~cm})$ \\
Vertical Aperture & $26 "(66 \mathrm{~cm})$ & $70 "(178 \mathrm{~cm})$ \\
Field Integral & $8.14 \mathrm{Tm}$ & $3.0 \mathrm{Tm}$ \\
Ampere-Turns & 670,000 & 800,000 \\
Current & $2,400 \mathrm{Amp}$ & $4,200 \mathrm{Amp}$ \\
Power & $580 \mathrm{kWatt}$ & $400 \mathrm{kWatt}$ \\
Inlet Water Temperature & $38^{\circ} \mathrm{C}$ & $38^{\circ} \mathrm{C}$ \\
Temperature Rise & $25^{\circ} \mathrm{C}$ & $25^{\circ} \mathrm{C}$ \\
Water Flow & $90 \mathrm{gal} / \mathrm{min}$ & $60 \mathrm{gal} / \mathrm{min}$ \\
Weight: & & \\
Pole Inserts & $9.5 \mathrm{t}$ & $10 \mathrm{t}$ \\
Coils & $19 \mathrm{t}$ & $40 \mathrm{t}$ \\
Return Yoke & $420 \mathrm{t}$ & $300 \mathrm{t}$ \\
Total & $450 \mathrm{t}$ & $350 \mathrm{t}$ \\
\hline
\end{tabular}

Table 4.3: The characteristics of the first magnet, FMAG, and the second magnet, KMAG. 

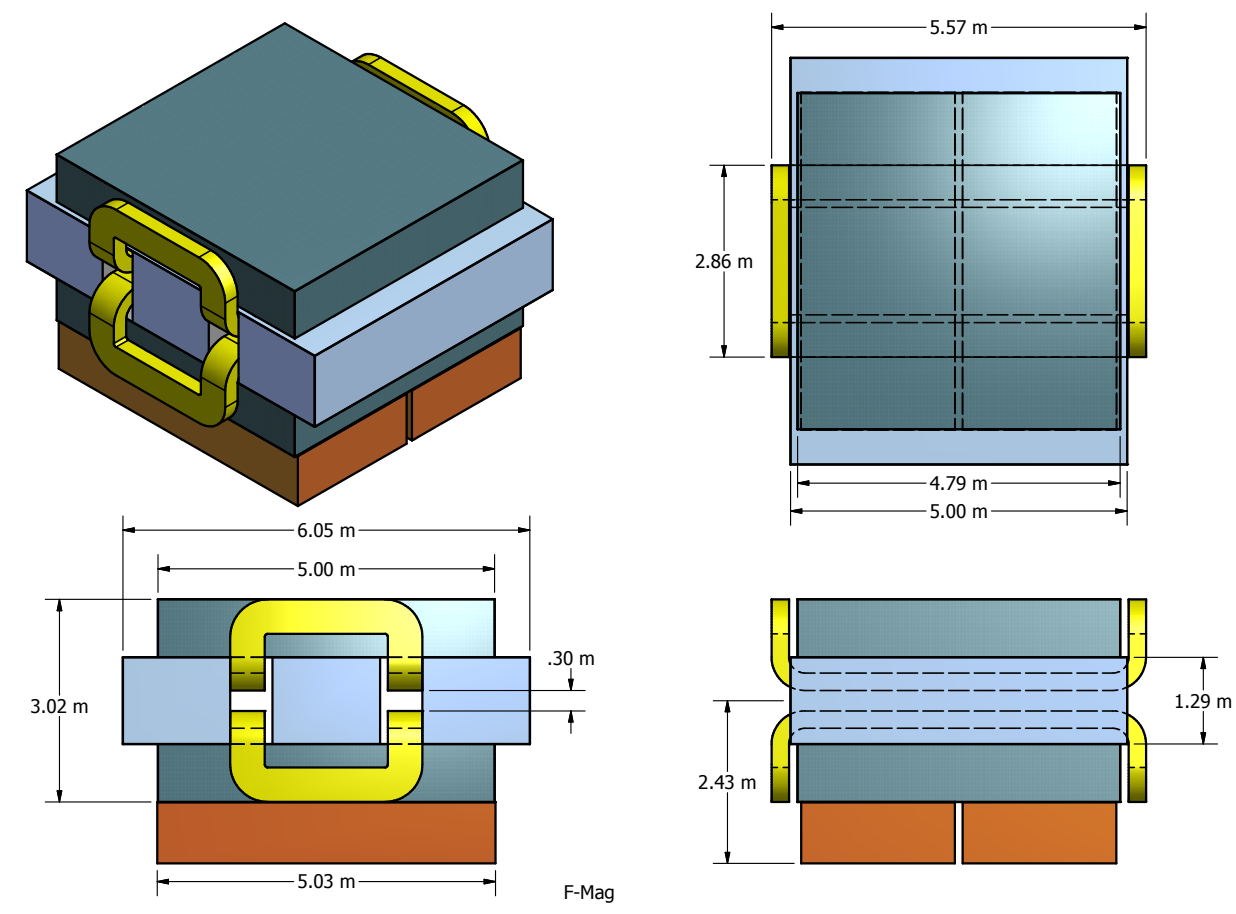

Figure 4.7: Drawing of FMAG [2.9]. This magnet focuses the high momentum muons into the acceptance of the apparatus and bends low momentum muons out of the acceptance. A beam dump is also placed inside the magnet to stop the beam. 

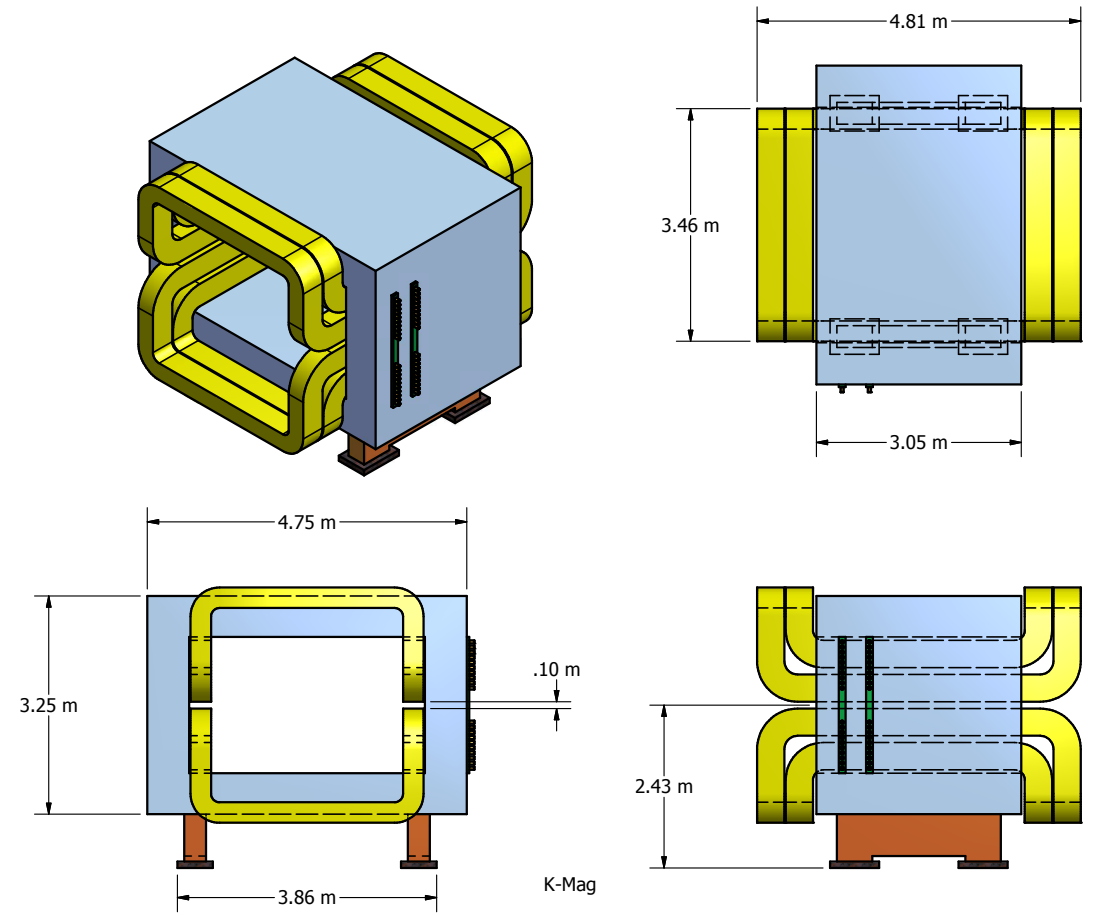

Figure 4.8: Drawing of KMAG [2.9]. This magnet is used to measure the momentum of muons.

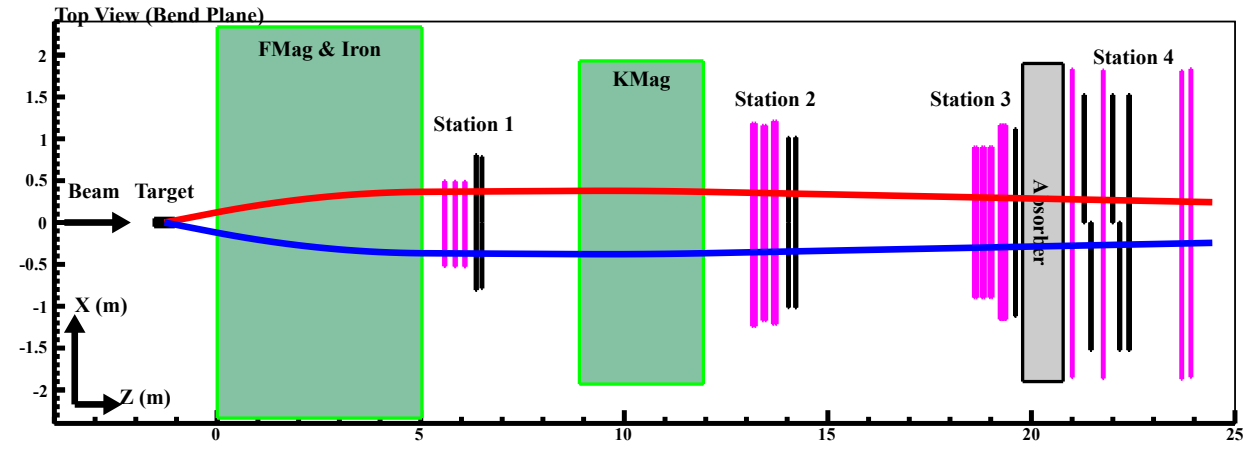

Figure 4.9: Bend plane view (top view) of spectrometer with muon tracks. Red and blue lines show tracks of positive and negative muons penetrating into the spectrometer, respectively. 


\subsection{Detector stations}

\subsubsection{Scintillator Hodoscopes}

Scintillator hodoscope planes are located at each tracking station. Those Hodoscopes are categorized as " $x$-measuring hodoscopes" or " $y$-measuring hodoscopes", according to their paddles' orientation. The drawings of the hodoscopes are found in Fig. 4.10-4.T3. The $x$ - $(y-)$ measuring hodoscopes have paddles oriented vertically (horizontally) and are sensitive to measure $x-(y-)$ position of penetrating muons. The detailed specifications of all the hodoscope planes are found in Table 4.4. Each of these hodoscopes is explained as following.

\section{Station 1 Hodoscope}

Station 1 has both $x$-measuring hodoscopes and $y$-measuring hodoscopes as shown in Fig. 4.10. Each hodoscope plane is split into two half-planes of parallel scintillator paddles (top and bottom for $x$-measuring hodoscopes, or left and right for $y$-measuring hodoscopes). Each hodoscope plane is named according to the tracking station to which it belongs, together with its position such as, "T" or "B" ("L" or "R"). For example, "H1T hodoscope" refers to the Station 1 hodoscope plane in which scintillator detectors are positioned vertically on the top side.

\section{Station 2 Hodoscope}

Station 2 has also both $x$-measuring hodoscopes and $y$-measuring hodoscopes as shown in Fig. 4.1 like Station 1. Each hodoscope plane is split into two half-planes of parallel scintillator paddles. The H2L and H2R hodoscopes are overlapped at the end of paddles.

\section{Station 3 Hodoscope}

Station 3 has only $x$-measuring hodoscopes. The drawing is shown in Fig. 4.12. The hodoscope plane is split into two half-planes of parallel scintillator paddles as well.

\section{Station 4 Hodoscope}

Station 4 has one plane of $x$-measuring hodoscopes and two planes of $y$-measuring hodoscopes. The drawings are shown in Fig. 4.13. Two planes of $y$-measuring hodoscopes are called "H4Y1" and "H4Y2", respectively. The scintillator paddles in Station 4 are read from both side due to the long paddles covering a large detection area while the 
scintillator paddles in the other Stations are read from one side. There are gaps at the both outer edge of $y=0$ in order to reduce hit rates on the hodoscopes.

\begin{tabular}{llllllll}
\hline \hline Detector & $\begin{array}{l}\text { Width } \\
(\mathrm{cm})\end{array}$ & $\begin{array}{l}\text { Overlap } \\
(\mathrm{cm})\end{array}$ & $\begin{array}{l}\text { \# of } \\
\text { paddles }\end{array}$ & $\begin{array}{l}x \\
(\mathrm{~cm})\end{array}$ & $\begin{array}{l}y \\
(\mathrm{~cm})\end{array}$ & $\begin{array}{l}z \text {-position } \\
(\mathrm{cm})\end{array}$ \\
\hline H1T & 7.32 & 0.32 & 23 & 162.00 & $\times$ & 69.85 & 667.12 \\
H1B & 7.32 & 0.32 & 23 & 162.00 & $\times$ & 69.85 & 667.12 \\
H1L & 7.32 & 0.32 & 20 & 78.74 & $\times$ & 140.12 & 654.03 \\
H1R & 7.32 & 0.32 & 20 & 78.74 & $\times$ & 140.12 & 654.03 \\
H2T & 13.04 & 0.32 & 16 & 203.24 & $\times$ & 152.00 & 1421.06 \\
H2B & 13.04 & 0.32 & 16 & 203.24 & $\times$ & 152.00 & 1421.06 \\
H2L & 13.07 & 0.32 & 19 & 132.00 & $\times$ & 241.29 & 1402.86 \\
H2R & 13.07 & 0.32 & 19 & 132.00 & $\times$ & 241.29 & 1402.86 \\
H3T & 14.59 & 0.32 & 16 & 227.52 & $\times$ & 167.64 & 1958.51 \\
H3B & 14.59 & 0.32 & 16 & 227.52 & $\times$ & 167.64 & 1958.51 \\
H4T & 19.65 & 0.32 & 16 & 304.52 & $\times$ & 182.88 & 2234.50 \\
H4B & 19.65 & 0.32 & 16 & 304.52 & $\times$ & 182.88 & 2250.68 \\
H4Y1L & 23.48 & 0.32 & 16 & 152.40 & $\times$ & 365.80 & 2130.27 \\
H4Y1R & 23.48 & 0.32 & 16 & 152.40 & $\times$ & 365.80 & 2146.45 \\
H4Y2L & 23.48 & 0.32 & 16 & 152.40 & $\times$ & 365.80 & 2200.44 \\
H4Y2R & 23.48 & 0.32 & 16 & 152.40 & $\times$ & 365.80 & 2216.62 \\
\hline
\end{tabular}

Table 4.4: Specifications of SeaQuest hodoscope planes. Width shows the width of each scintillator paddle in hodoscope plane. $x$ and $y$ show width and height of each hodoscope plane, respectively. 


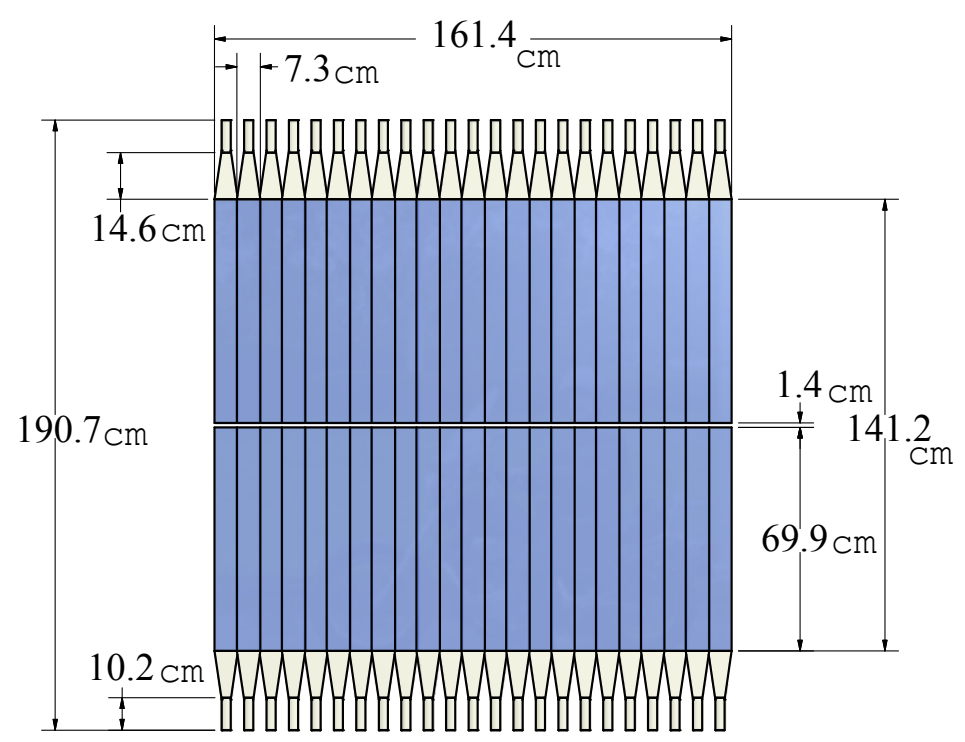

Hodoscope Array $1 \mathrm{X}$

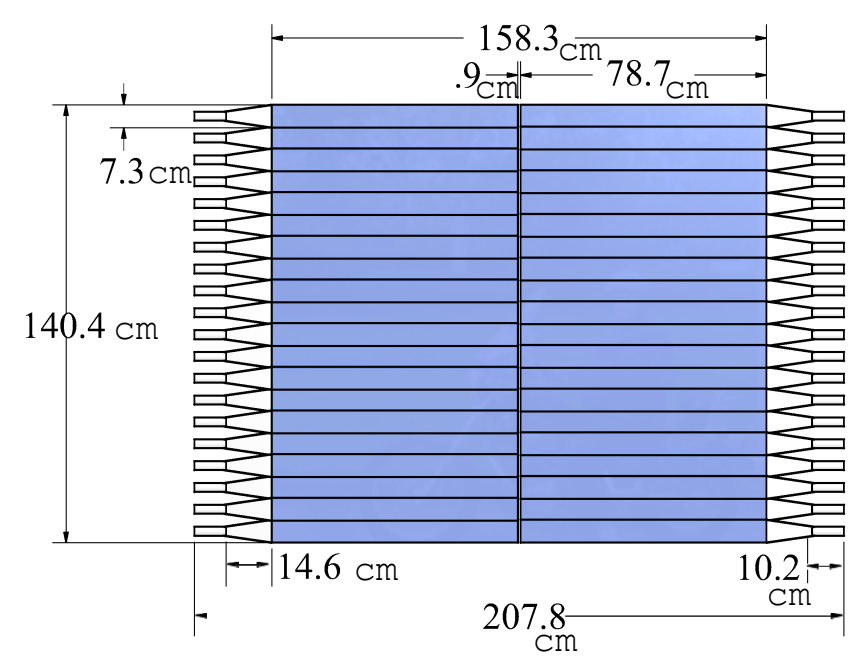

Hodoscope Array $1 Y$

Figure 4.10: Drawing of scintillator hodoscopes [2.9] located at the first tracking station, named H1X, H1Y. Each hodoscope plane is split into two half planes of parallel scintillator paddles; top and bottom for $x$-measuring hodoscopes (top figure), or left and right for $y$-measuring hodoscopes (bottom figure). 

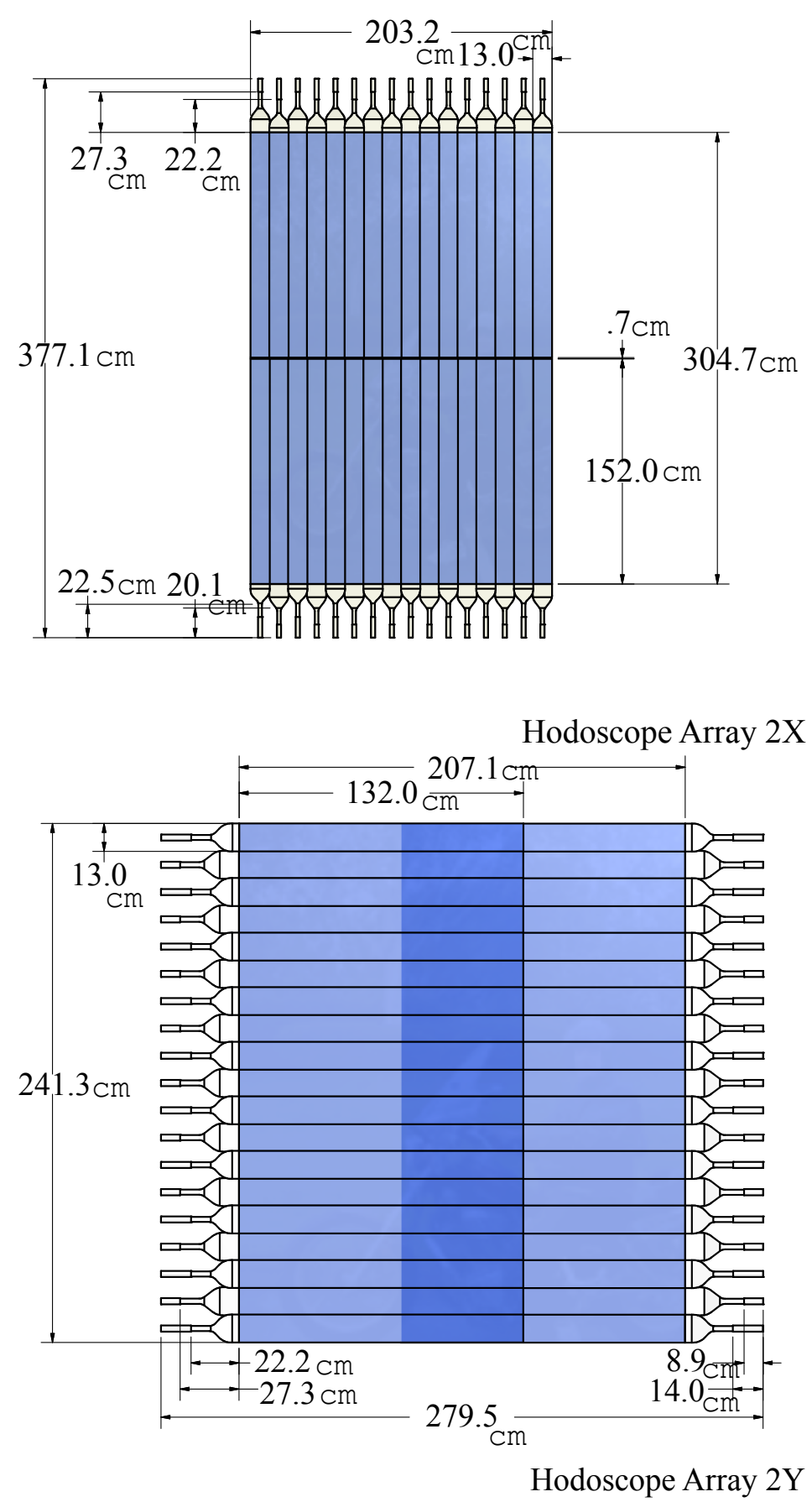

Figure 4.11: Drawing of scintillator hodoscopes [2.9] located at the second tracking station, named H2X, H2Y. Each hodoscope plane is split into two half planes of parallel scintillator paddles; top and bottom for $x$-measuring hodoscopes (top figure), or left and right for $y$-measuring hodoscopes (bottom figure). 


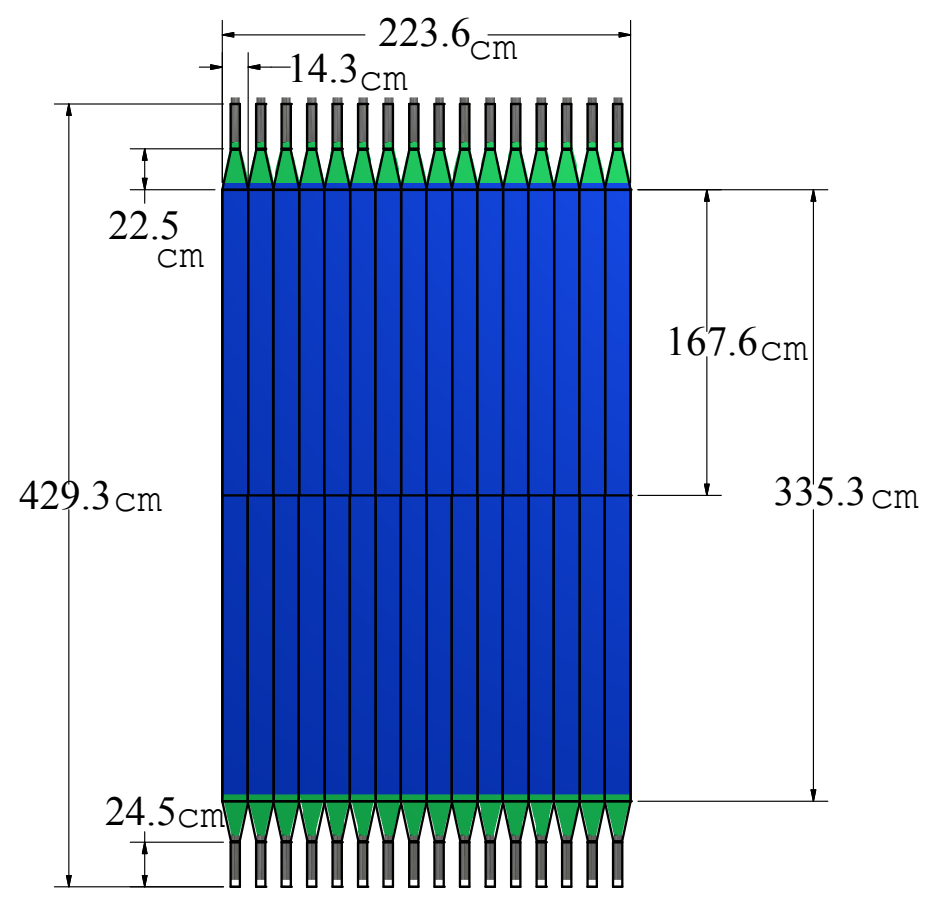

Hodoscope Array 3X

Figure 4.12: Drawing of scintillator hodoscopes [2.9] located at the third tracking station, named H3X. Only x-direction scintillator hodoscopes array is located in this tracking station. The hodoscope plane is split into two half planes of parallel scintillator paddles, top and bottom. 

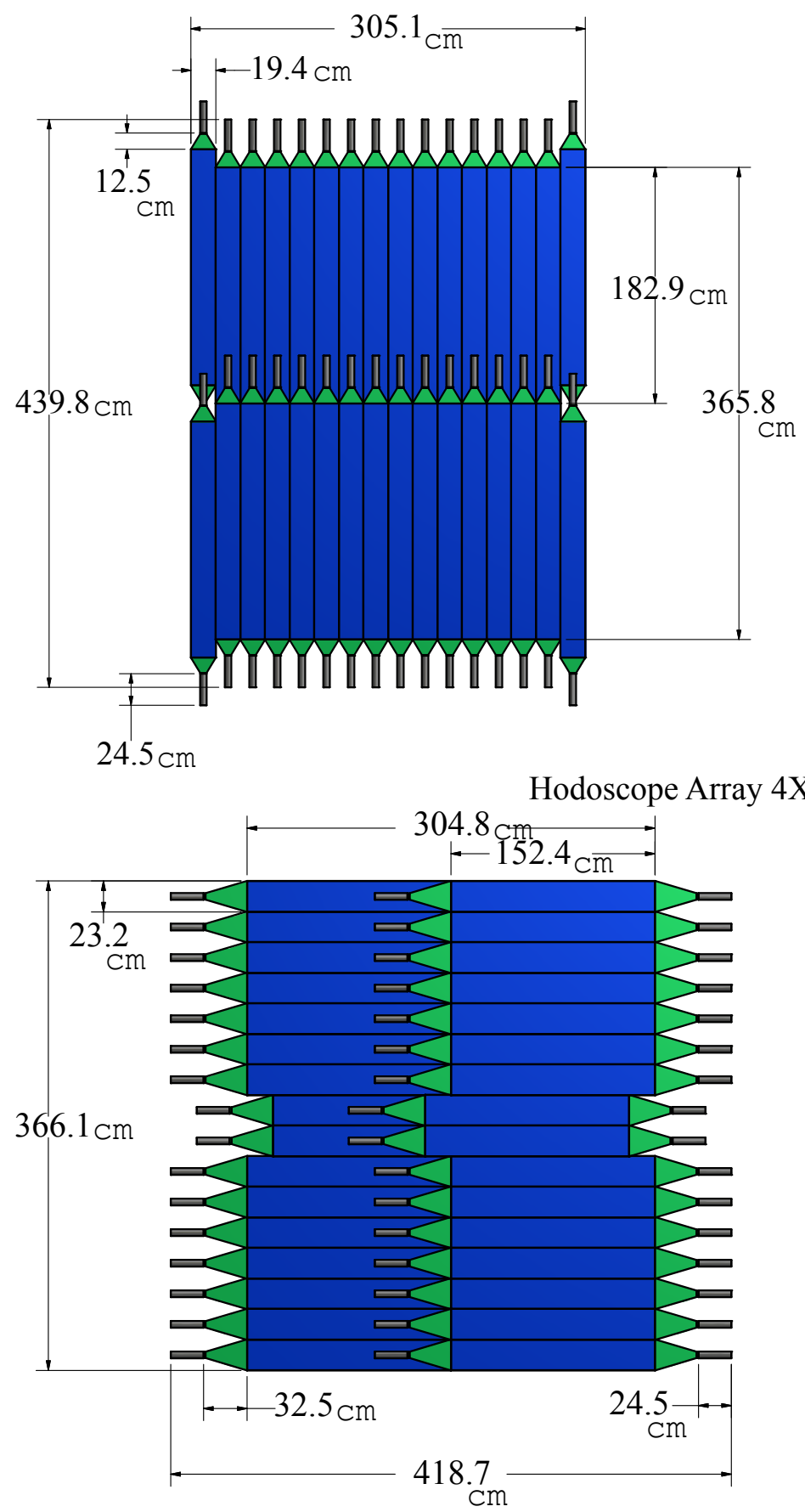

Hodoscope Array 4Y

Figure 4.13: Drawing of scintillator hodoscopes [2.9] located at the fourth tracking station, named H4X, H4Y. Each hodoscope plane is split into two half planes of parallel scintillator paddles; top and bottom for $x$-measuring hodoscopes (top figure), or left and right for $y$ measuring hodoscopes (bottom figure). Hodoscopes in Station 4 have scintillator paddles read from both side due to the long paddles covering a large detection area. 


\subsubsection{Drift Chambers}

Each of Station 1 and Station 2 has one drift chamber. Station 3 has two drift chambers in order to cover large detection area. One of them covers the upper region than the beamline while the other covers the lower region than the beamline. The upper(lower) one is called Station 3 plus(minus) drift chamber.

All of the drift chambers have wires stretched in the vertical direction. It is because the penetrating muons are bent in the horizontal direction by the magnets. All the drift chambers have a common structure. They have 6 tracking planes that are U, U', X, X', $\mathrm{V}$, and $\mathrm{V}^{\prime}$ planes. $\mathrm{U}$ and $\mathrm{U}^{\prime}$ planes have wires which are tilted approximately by $+14^{\circ}$ $(\tan \theta=0.25)$ from the vertical direction in the $x-y$ plane. $\mathrm{X}$ and $\mathrm{X}$ ' planes have wires which are vertical. $\mathrm{V}$ and $\mathrm{V}^{\prime}$ planes have wires which are tilted approximately by $-14^{\circ}$ $(\tan \theta=-0.25)$ in the $x-y$ plane. The sense wires of $\mathrm{U}^{\prime}, \mathrm{X}^{\prime}$ and $\mathrm{V}^{\prime}$ planes are shifted by half a drift cell as compared to $\mathrm{U}, \mathrm{X}$ and $\mathrm{V}$ planes to help resolve the ambiguity of the drift direction. Each drift chamber plane is named according to the tracking station to which it belongs, together with its plane name. For example, "D3pXp" refers to the $\mathrm{X}$ ' plane of the upper drift chamber in the Station 3. The detection areas of the drift chambers in $x-y$ plane are shown in Fig. 4.14. Station 1, 2, 3 plus and 3 minus are shown from top to bottom. Figures in the left column show the X-planes, and figures in the right column show the tilted U-planes. The $\mathrm{V}$-planes have a mirrored structure of the U-planes. Drift chambers used in E866 experiment are reused for Station 1 and 2. Station 3 plus (upper region) drift chamber was newly fabricated in Japan by the Japanese SeaQuest collaboration and was shipped from Japan to Fermilab. Another E866 drift chamber had been reused as Station 3 minus (lower region) drift chamber for the detector commissioning period. Later, a new drift chamber was constructed by SeaQuest collaborator in Fermilab under the initiative of the Japanese group during the detector upgrade period and it has been used from Run II. The chamber has completely the same design as Station 3 plus drift chamber. The drawing of the Station 3 chambers are shown in Fig. 4.15. All the drift chambers are operated with a gas mixture of P08 and $\mathrm{CF}_{4}$ (argonne:methane: $\mathrm{CF}_{4} \simeq 88 \%: 8 \%: 4 \%$ ). The detailed specifications of all the drift chamber planes are found in Table 4.5. 


\begin{tabular}{llllllll}
\hline \hline Detector & $\begin{array}{l}\text { cell size } \\
(\mathrm{cm})\end{array}$ & \# of cells & $\begin{array}{l}\text { tilt angle } \\
(\mathrm{rad})\end{array}$ & $\begin{array}{l}\text { width } \\
(\mathrm{cm})\end{array}$ & $\begin{array}{l}\text { height } \\
(\mathrm{cm})\end{array}$ & $\begin{array}{l}\text { z-position } \\
(\mathrm{cm})\end{array}$ \\
\hline D1U & 0.635 & 201 & 0.245 & 101.60 & $\times$ & 121.92 & 594.49 \\
D1Up & 0.635 & 201 & 0.245 & 101.60 & $\times$ & 121.92 & 595.13 \\
D1X & 0.635 & 160 & 0 & 101.60 & $\times$ & 121.92 & 617.09 \\
D1Xp & 0.635 & 160 & 0 & 101.60 & $\times$ & 121.92 & 617.72 \\
D1V & 0.635 & 201 & -0.245 & 101.60 & $\times$ & 121.92 & 637.17 \\
D1Vp & 0.635 & 201 & -0.245 & 101.60 & $\times$ & 121.92 & 637.81 \\
\hline D2V & 2.021 & 128 & -0.245 & 233.27 & $\times$ & 264.16 & 1314.98 \\
D2Vp & 2.021 & 128 & -0.245 & 233.27 & $\times$ & 264.16 & 1321.96 \\
D2Xp & 2.083 & 112 & 0 & 233.27 & $\times$ & 264.16 & 1340.36 \\
D2X & 2.083 & 112 & 0 & 233.27 & $\times$ & 264.16 & 1347.34 \\
D2U & 2.021 & 128 & 0.245 & 233.27 & $\times$ & 264.16 & 1365.99 \\
D2Up & 2.021 & 128 & 0.245 & 233.27 & $\times$ & 264.16 & 1372.98 \\
\hline D3pVp & 2.000 & 134 & 0.245 & 320.00 & $\times$ & 166.00 & 1923.33 \\
D3pV & 2.000 & 134 & 0.245 & 320.00 & $\times$ & 166.00 & 1925.33 \\
D3pXp & 2.000 & 116 & 0 & 320.00 & $\times$ & 166.00 & 1929.33 \\
D3pX & 2.000 & 116 & 0 & 320.00 & $\times$ & 166.00 & 1931.33 \\
D3pUp & 2.000 & 134 & -0.245 & 320.00 & $\times$ & 166.00 & 1935.33 \\
D3pU & 2.000 & 134 & -0.245 & 320.00 & $\times$ & 166.00 & 1937.33 \\
\hline D3mVp & 2.000 & 134 & 0.245 & 320.00 & $\times$ & 166.00 & 1886.77 \\
D3mV & 2.000 & 134 & 0.245 & 320.00 & $\times$ & 166.00 & 1888.77 \\
D3mXp & 2.000 & 116 & 0 & 320.00 & $\times$ & 166.00 & 1892.77 \\
D3mX & 2.000 & 116 & 0 & 320.00 & $\times$ & 166.00 & 1894.77 \\
D3mUp & 2.000 & 134 & -0.245 & 320.00 & $\times$ & 166.00 & 1898.77 \\
D3mU & 2.000 & 134 & -0.245 & 320.00 & $\times$ & 166.00 & 1900.77 \\
\hline
\end{tabular}

Table 4.5: Specification of all the drift chambers in the SeaQuest spectrometer. 
D1-X

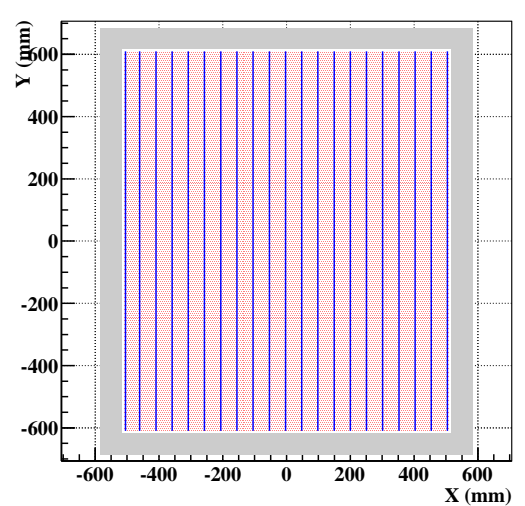

D2-X

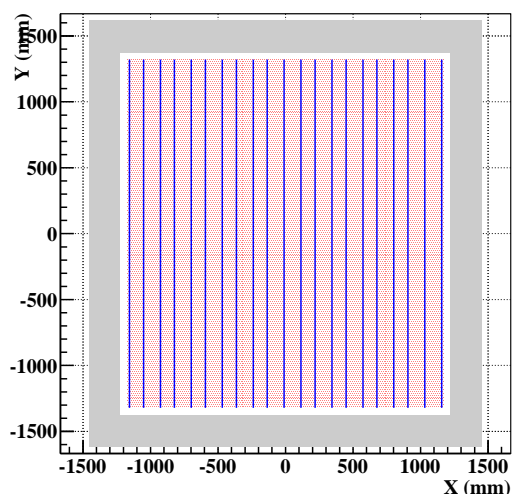

D3p-X

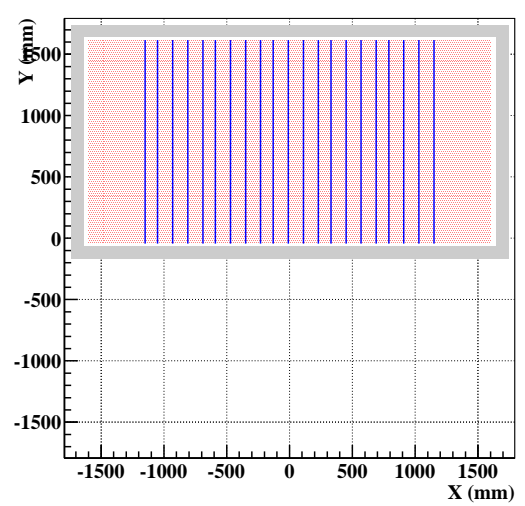

D3m-X

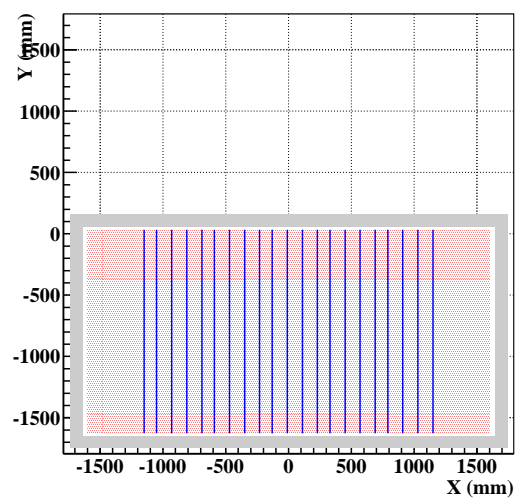

D1-U

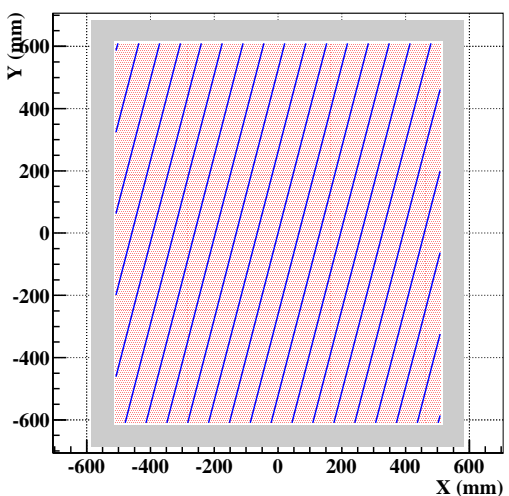

D2-U

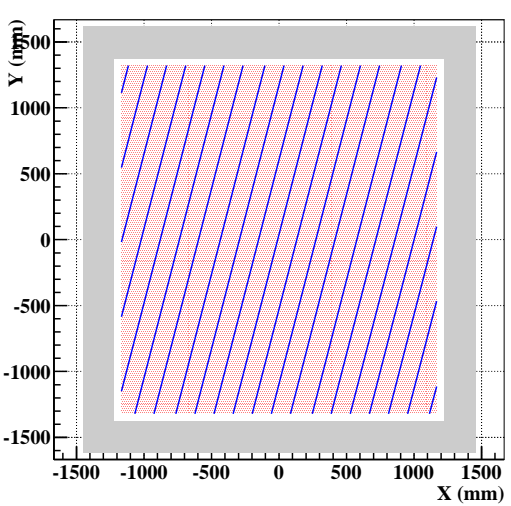

D3p-U

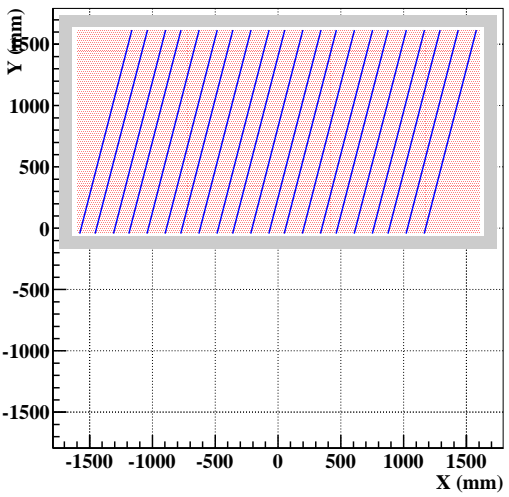

D3m-U

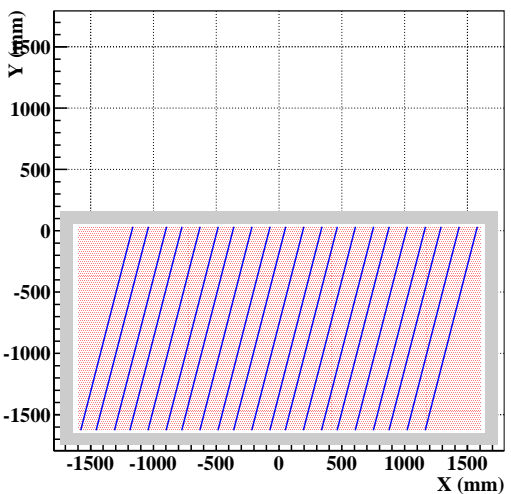

Figure 4.14: Overview of the four different drift chambers used in the SeaQuest experiment. Station 1, 2, 3 plus and 3 minus are shown from top to bottom in the $x-y$ plane. Left column shows the X-planes, right column the tilted U-planes. The V-planes has a mirrored structure of the U-planes. 


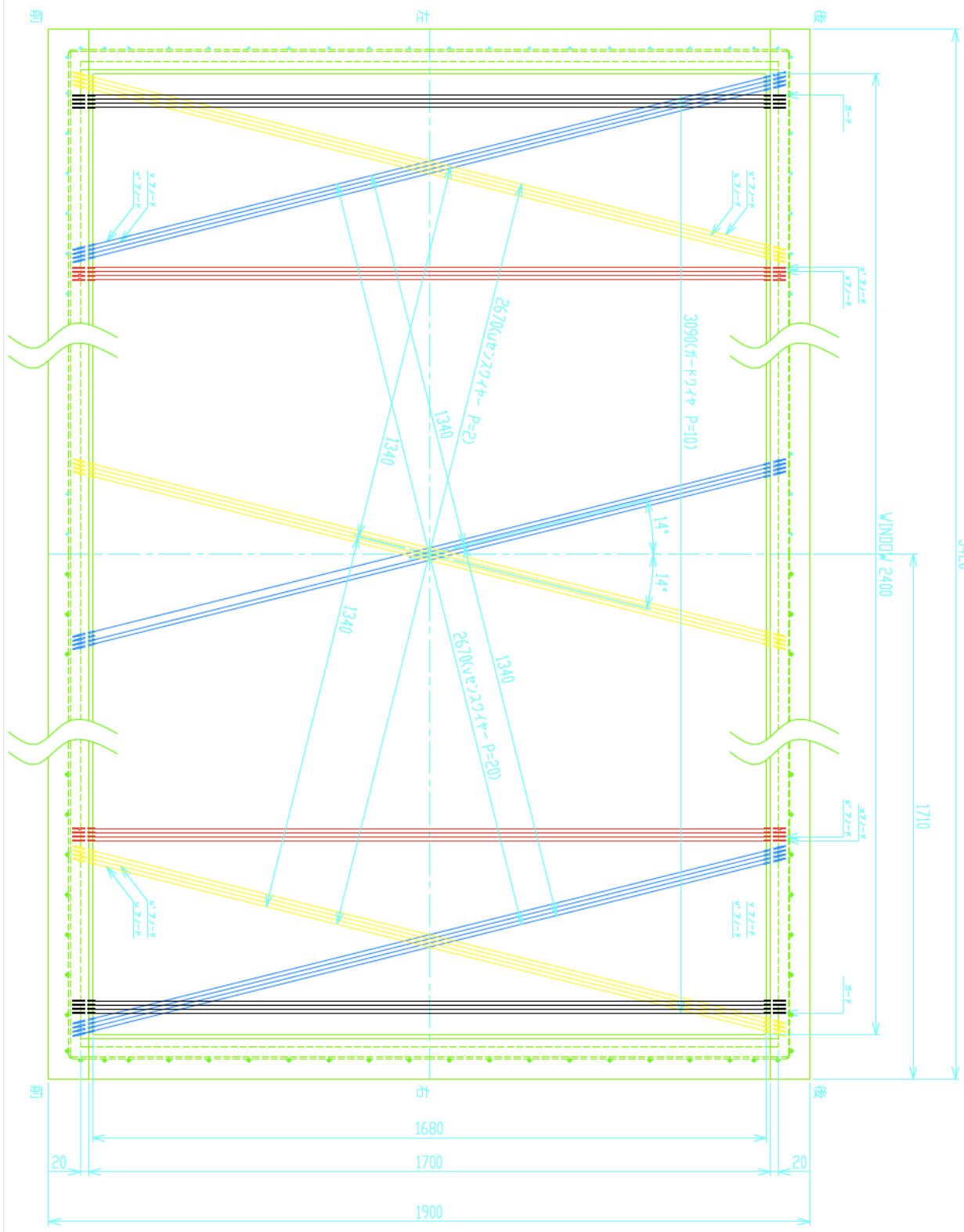

Figure 4.15: Drawing of St. 3 drift chamber (front view). The size is written in $\mathrm{mm}$. It is tilted by 90 degrees in this figure. The size of the chamber is $3.42 \mathrm{~m} \times 1.90 \mathrm{~m}$. The size of its effective area is $2.22 \mathrm{~m} \times 1.60 \mathrm{~m}$. 


\subsubsection{Structure of SeaQuest drift chambers}

\section{Chamber structure}

Figure 4.15 shows the drawing of the Station 3 drift chambers (Station 3 plus \& minus). These Station 3 chambers have all the six planes combined in one chamber box while the other drift chambers (Station 1 and 2 chambers) have three separated chamber box, respectively, where each box has paired plane (U/U' or $\mathrm{X} / \mathrm{X}^{\prime}$ or $\left.\mathrm{V} / \mathrm{V}^{\prime}\right)$.

Station 3 chambers have "feed-throughs" which help to position wires with a high precision on the side frame. Figure 4.16 shows the feed-throughs lined and attached on the chamber side frame of the Station 3 chamber. The right picture is a close-up photo. The feed-throughs for the sense wires are marked by red circles. The feed-through consists of three parts; a metal part, a plastic part, and a positioning bush. A wire is soldered to the metal part. The plastic part holds the metal part and insulates it from the chamber frame. The positioning bush which is embedded inside the plastic part in fact positions a wire with a high precision. The other chambers have G10 frames with electronic circuit soldered on it, and wires are soldered on the circuit. Each type, feed-through type and soldered circuit type, has its advantage:

- Feed-through type

- Feed-throughs position wires with a high precision.

- Broken wires can be fixed without opening chamber. (But the procedure is complex.)

- Chamber structure can be simple.

- Soldered circuit type

- No need to assemble readout and high voltage guides because of the circuit already printed on the frame.

- It is usually easier to replace wires if broken, than the feed-through type, although it depends on the situation. 

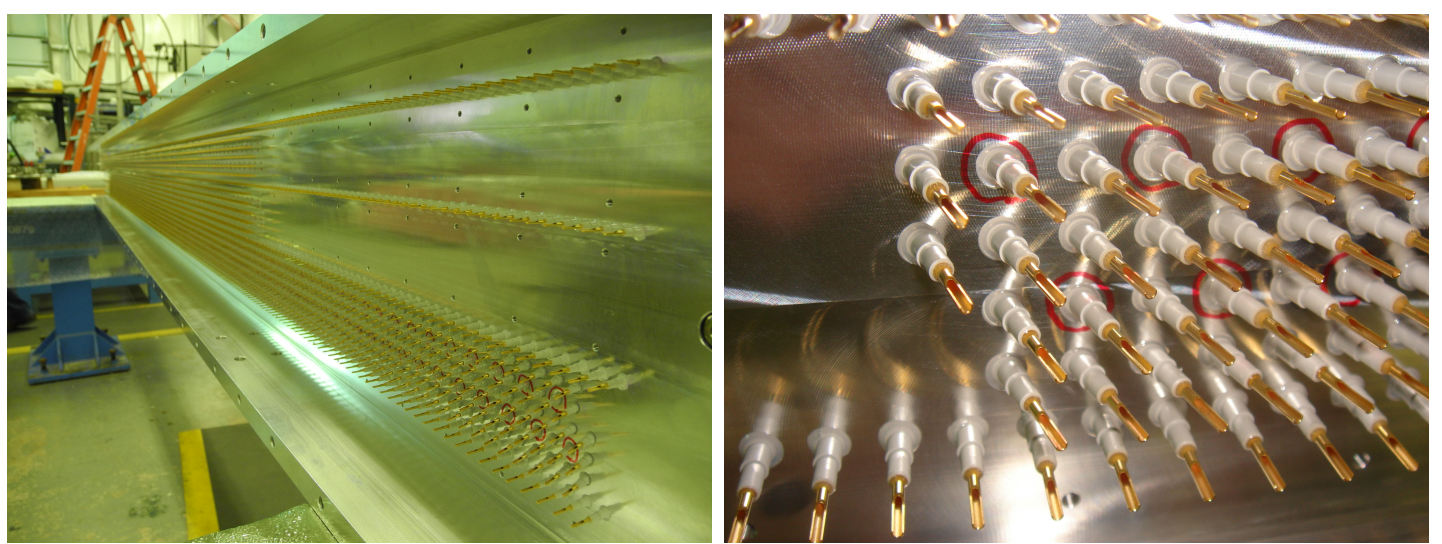

Figure 4.16: Feed-throughs lined and attached on the side frame of the Station 3 chamber. Right picture is a close-up photo. Each feed-through helps to position a wire with a high position resolution. The feed-throughs for the sense wires are marked by red circles. 

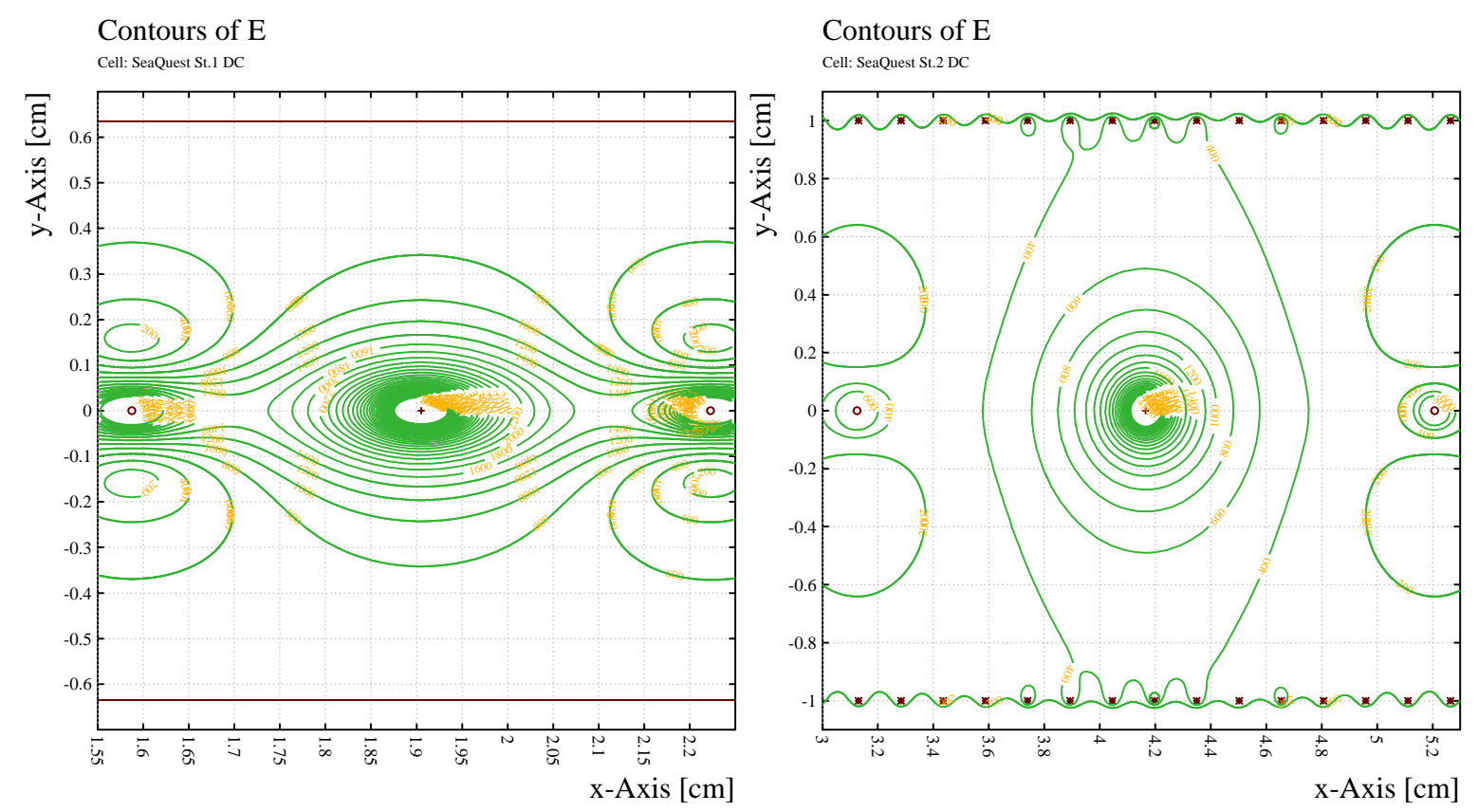

Figure 4.17: Field map in one cell. The left figure shows the contour of the magnitude of electric field $E$ of Station 1 drift chamber, and the right figure shows that of Station 2 drift chamber.

\section{Cell Structure of Station 1 drift chamber}

The cell structure of Station 1 drift chamber is shown in the left figure of Fig. 4.17. It shows a field map in one cell. It shows the contour of the magnitude of electric field $E$. Field wires and sense wires are stretched alternately in a sense wire plane, $y=0$, in the figure. The sense wire plane is sandwiched by two cathode planes. The field wires and cathode planes are grounded. Positive high voltages (typical value is $\sim+1.5 \mathrm{kV}$ ) are applied to the sense wires. Table 4.6 summarizes the characteristics of wires or plane of Station 1 drift chamber.

\begin{tabular}{llll}
\hline \hline Wire or plane & Material & Diameter $(\mu \mathrm{m})$ & Typical value of HV $(\mathrm{V})$ \\
\hline Sense & Gold-coated tungsten & 25 & +1500 \\
Field & Beryllium-copper & 62.5 & 0 \\
Cathode & Aluminum Mylar & - & 0 \\
\hline
\end{tabular}

Table 4.6: Characteristics of wires or plane of Station 1 drift chamber. Material, diameter, and typical value of high voltages (HV) are listed for each wire and plane. 


\section{Cell Structure of Station 2 drift chamber}

The cell structure of Station 2 drift chamber is shown in the right figure of Fig. 4.17. It shows a field map in one cell. Field wires and sense wires are stretched alternately in a sense wire plane, $y=0$, in the figure. Cathode wires are lined at $y= \pm 1 \mathrm{~cm}$ to sandwich the sense wire plane. Typically $-1850 \mathrm{~V}$ is applied to the field and cathode wires. The sense wires are grounded. Table 4.7 summarizes the characteristics of wires of Station 2 drift chamber.

\begin{tabular}{llll}
\hline \hline Wire & Material & Diameter $(\mu \mathrm{m})$ & Typical value of HV $(\mathrm{V})$ \\
\hline Sense & Gold-coated tungsten & 25 & 0 \\
Field & Beryllium-copper & 62.5 & -1850 \\
Cathode & Beryllium-copper & 62.5 & -1850 \\
\hline
\end{tabular}

Table 4.7: Characteristics of wires of Station 2 drift chamber. Material, diameter, and typical value of high voltages (HV) are listed for each wire.

\section{Cell Structure of Station 3 drift chambers}

The wire cell structure of the Station 3 chambers is shown in Fig. 4.18. A sense wire is surrounded by eight of cathode or field wires. Guard wires are lined between plane pairs and outside of $\mathrm{U} / \mathrm{U}^{\prime}$ and $\mathrm{V} / \mathrm{V}^{\prime}$ planes. Also, the field map in one cell is shown in Fig. 4.1.9. Typically $-2400 \mathrm{~V}$ is applied to the field and cathode wires, and $-1300 \mathrm{~V}$ is applied to the guard wires. The sense wires are grounded. Table 4.8 summarizes the characteristics of wires of Station 3 drift chambers.

\begin{tabular}{llll}
\hline \hline Wire & Material & Diameter $(\mu \mathrm{m})$ & Typical value of HV $(\mathrm{V})$ \\
\hline Sense & Gold-coated tungsten & 30 & 0 \\
Field & Beryllium-copper & 80 & -2400 \\
Cathode & Beryllium-copper & 80 & -2400 \\
Guard & Beryllium-copper & 80 & -1300 \\
\hline
\end{tabular}

Table 4.8: Characteristics of wires of Station 3 drift chamber. Material, diameter, and typical value of high voltages (HV) applied to are listed for each wire. 


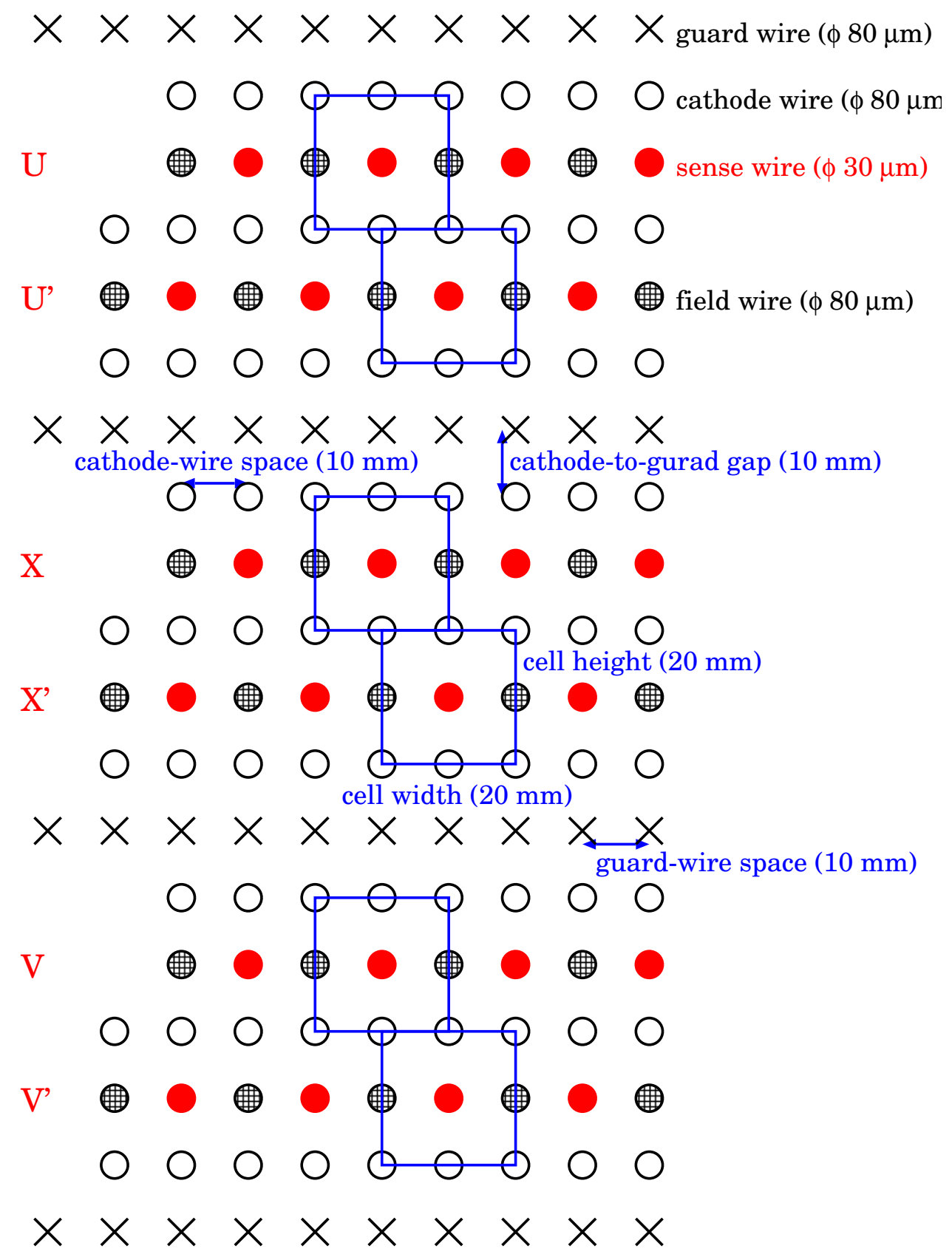

Figure 4.18: The wire cell structure of Station 3 drift chambers. This is the view of wire direction. Its structure is $2 \mathrm{~cm} \times 2 \mathrm{~cm}$ square. Sense wire (red circle) is positioned in the center of the cell, and surrounded by eight of field (gray circle) or cathode (white circle) wires. Guard wires (cross) are positioned out of each paired plane. 


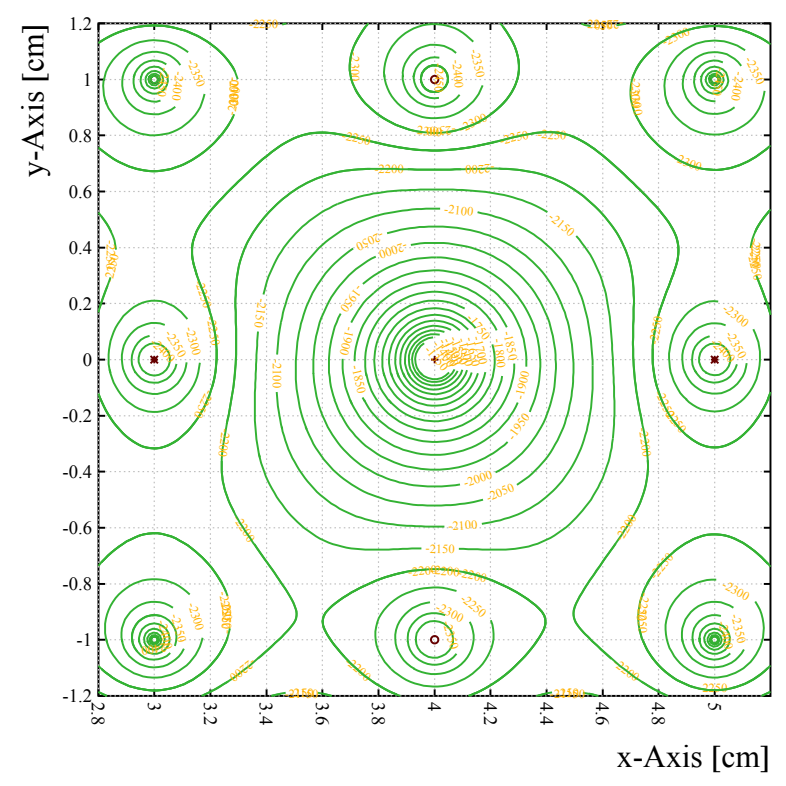

Figure 4.19: Field map in one cell of Station 3 drift chambers. The contour of the magnitude of electric field $E$ is shown. 


\subsubsection{Proportional Tubes}

Station 4 has proportional tubes to reconstruct muon tracks while other Stations have drift chambers. Each of the proportional tube plane has two layers of $5.08 \mathrm{~cm}$ wide cells. Paired layer was offset by half a cell size to cover dead region. These proportional tubes are named according to their tubes' orientation; ST4H or ST4V. The ST4H(V) proportional tubes are sensitive to measure the position of muons in the horizontal (vertical) axis. These proportional tubes are operated with the same gas mixture as used in the drift chambers. Typically, $+1800 \mathrm{~V}$ is applied to sense wires, and tubes are grounded. Existing amplifiers and discriminators from E866 are used as readout system. Detailed specifications of the proportional tubes are found in Table 4.9 .

\begin{tabular}{llllllll}
\hline \hline Detector & $\begin{array}{l}\text { tube radius } \\
(\mathrm{cm})\end{array}$ & \# of tubes & $\begin{array}{l}\text { tilt angle } \\
(\mathrm{rad})\end{array}$ & $\begin{array}{l}\mathrm{x} \\
(\mathrm{cm})\end{array}$ & $\begin{array}{l}\mathrm{y} \\
(\mathrm{cm})\end{array}$ & $\begin{array}{l}\text { Z-position } \\
(\mathrm{cm})\end{array}$ \\
\hline ST4H1a & 5.08 & 8 & 0 & 368.3 & $\times$ & 388.6 & 2102.1 \\
ST4V1a & 5.08 & 8 & $\pi$ & 388.6 & $\times$ & 368.3 & 2178.8 \\
ST4H2b & 5.08 & 8 & 0 & 368.3 & $\times$ & 388.6 & 2394.4 \\
ST4V2b & 5.08 & 8 & $\pi$ & 388.6 & $\times$ & 368.3 & 2371.3 \\
\hline
\end{tabular}

Table 4.9: Specifications of the proportional tubes. 

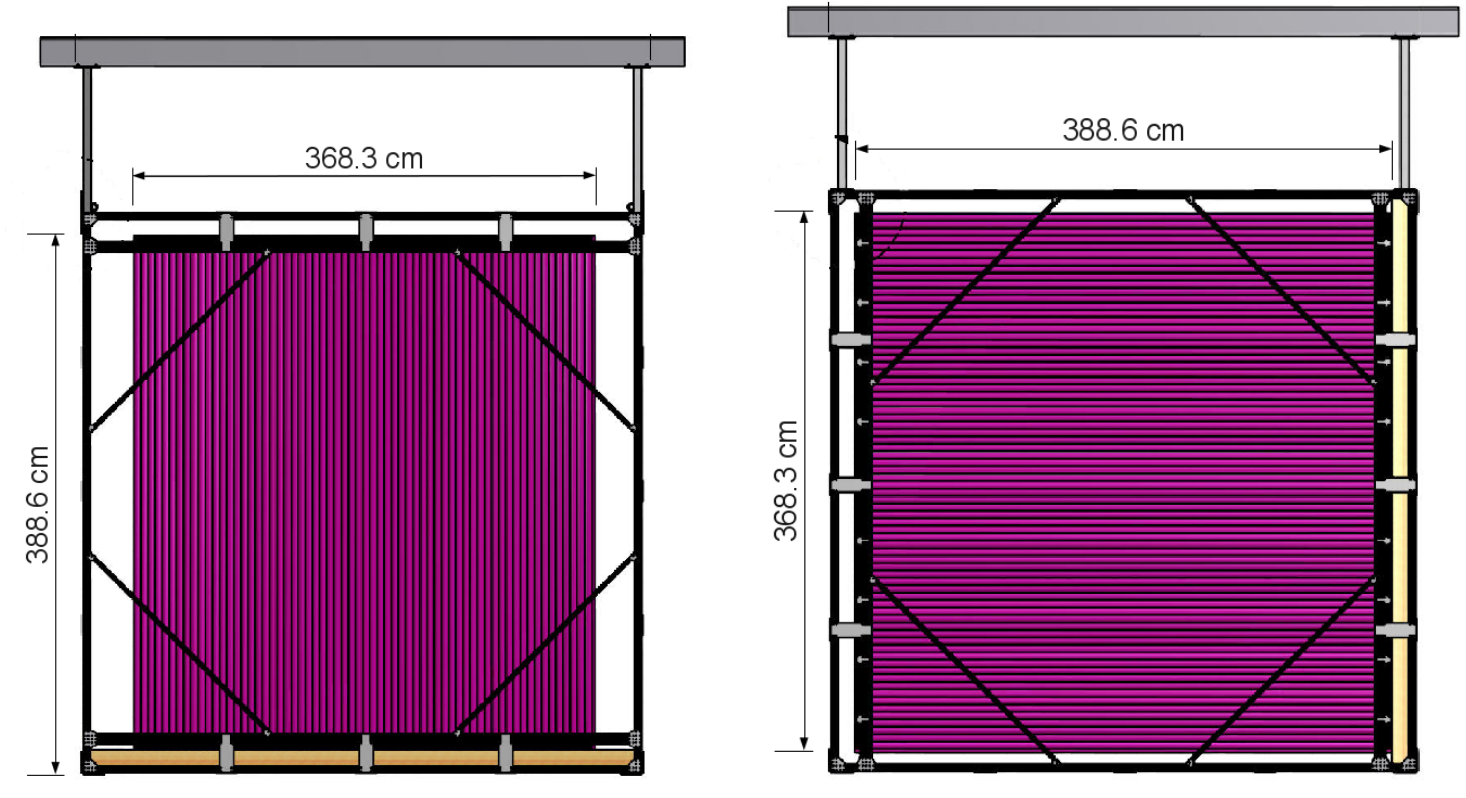

Figure 4.20: Drawing of proportional tubes. Left(right) shows $\mathrm{ST} 4 \mathrm{H}(\mathrm{V})$ proportional tubes. 


\subsection{Readout System for Detectors}

\subsubsection{TDC card}

The hodoscopes mainly provide the timing information while the drift chambers and proportional tubes provide the position and timing information of muons as they pass through the tracking station. The detectors are connected to time-to-digital converters (TDC). The TDC used in the SeaQuest experiment has $6 \mathrm{U}$ VMEbus form factor and is equipped with a low-power and radiation-hardened Microsemi ProASIC2 Flash base FPGA [30]. One TDC has 64-channels, and there are 89 TDCs being used to read out all the detectors in the SeaQuest spectrometer.

The TDCs receive two types of signals; the detectors' signals as 'start' signal, and trigger signal as 'stop' signal. Since the stop signal is common to all of the TDCs, it provides a reference against which the drift chamber and hodoscope arrival times should be compared. All the signal arrival time, and thus the time when the particles hit, for each detector can be compared to each other via this reference. The signals from hodoscopes are divided by NIM divider modules, and are sent to two different destinations. One is the TDCs where the timing of the hodoscope hits is recorded with respect to the stop signal. The other one is trigger system to create the trigger signal.

\subsubsection{ASDQ card}

Amplifiers are needed to read out the small raw signal from the chambers. SeaQuest uses amplifier cards called "ASDQ" card. "ASDQ" is an acronym for 'amplification', 'shaper', 'discriminator', and 'charge integration (Q option)'. All of these features are encoded in the "ASDQ chip" that was originally designed and developed for the central outer tracker of the CDF experiment at Fermilab [3]]. The ASDQ card has following features:

- Preamplifier: This step is responsible for amplifying the raw signal from drift chamber. It converts the charge input into a voltage output while minimizing noise added to the signal.

- Ion tail cancellation: In this step the tail of the amplified signal is eliminated. Also, the signal is amplified further more.

- Baseline restore (BLR): This step brings the baseline of the amplified signal to zero. This is the last stage where the analogue signal is handled before being discriminated by a discriminator. 


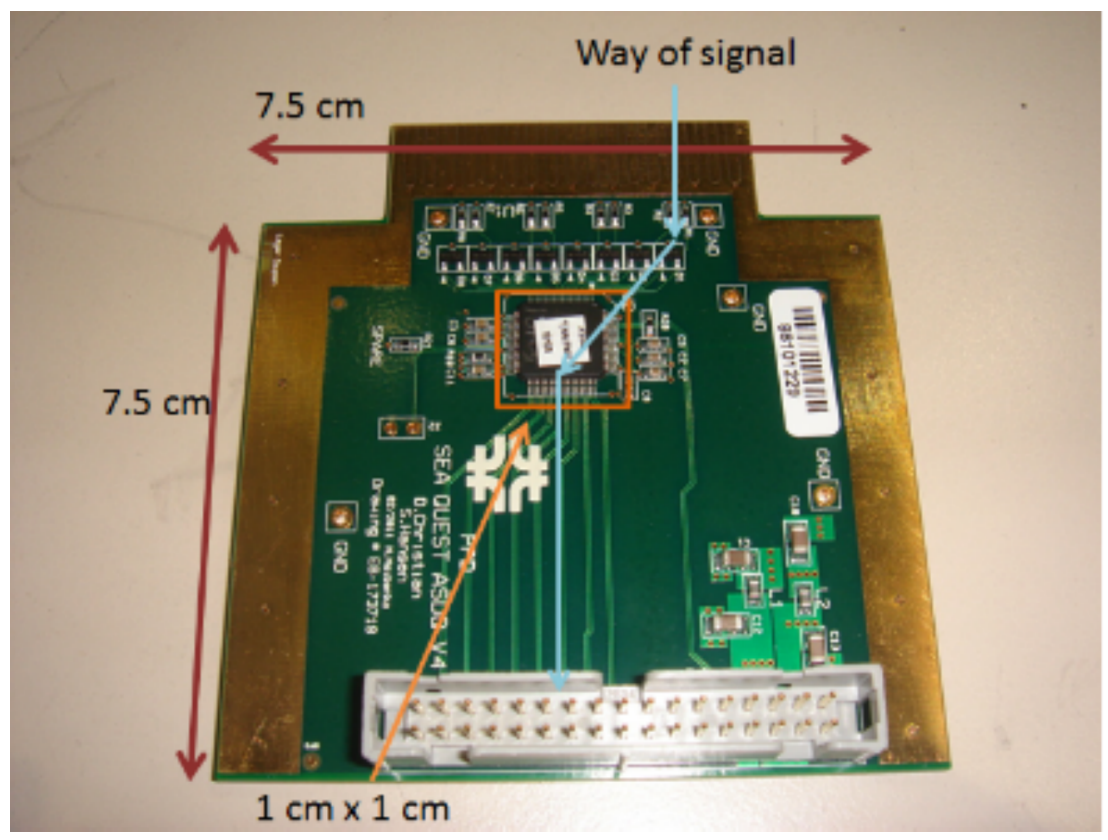

Figure 4.21: Picture of the ASDQ card. The ASDQ chip is placed on the center of the card and highlighted by an orange square.

- Discriminator: If the signal after the BLR step is above the user-selected threshold, a pair of differential digital signal is output. The threshold can be varied from 0 to $10 \mathrm{mV}$ for the amplified signal after the BLR step.

- Charge encoding option: This step makes the width of the digital output signal proportional to the total charge of the raw signal. This feature is not used in the SeaQuest experiment.

There is an input protection designed to buffer the preamplifier from large external spikes at the entrance of the ASDQ card. The picture of the ASDQ card is found in Fig. 4.27. There is an $1 \mathrm{~cm} \times 1 \mathrm{~cm}$ ASDQ chip mounted on the center of the card. The surrounding gold edge is at ground level, and the edge is connected to the frame of the drift chambers as shown in Fig. 4.22. One ASDQ card has 8 readout channels, each of which is connected to a sense wire. Thus, about 800 ASDQ cards are used for all the drift chambers in total.

\subsubsection{Level shifter board}

Level shifter board (LS board) is a specialized board to supply low voltages $(+3 \mathrm{~V}$ and $-3 \mathrm{~V}$ ) to the ASDQ cards to operate the cards (see Fig. 4.2:3). The differential digital 


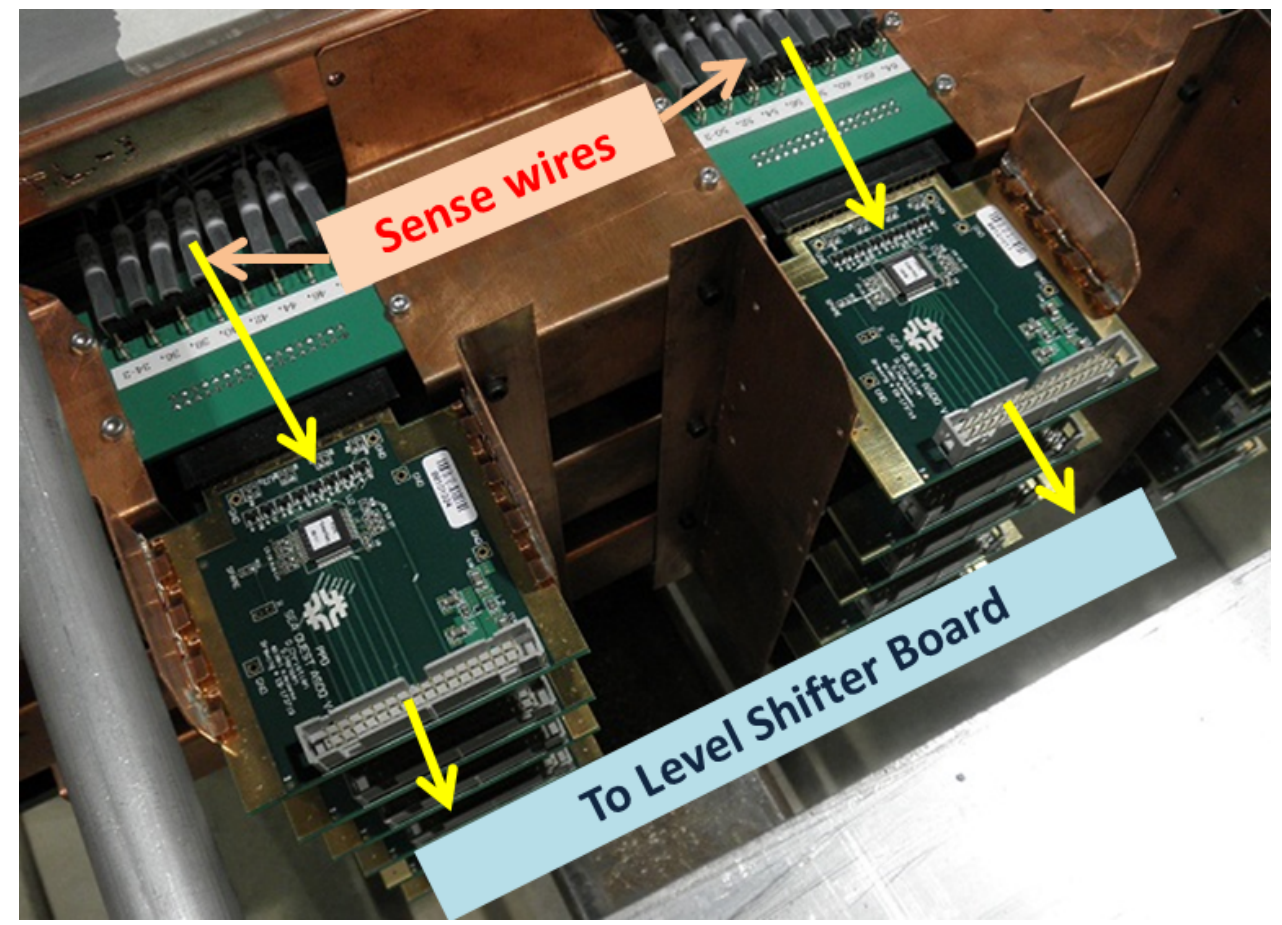

Figure 4.22: ASDQ cards connected to a drift chamber. 8 sense wires are connected to one ASDQ card. The cards are supported by copper parts connected to the chamber frame that serves as common ground to the electronics. Yellow arrows indicate the signal path starting from the sense wires. 


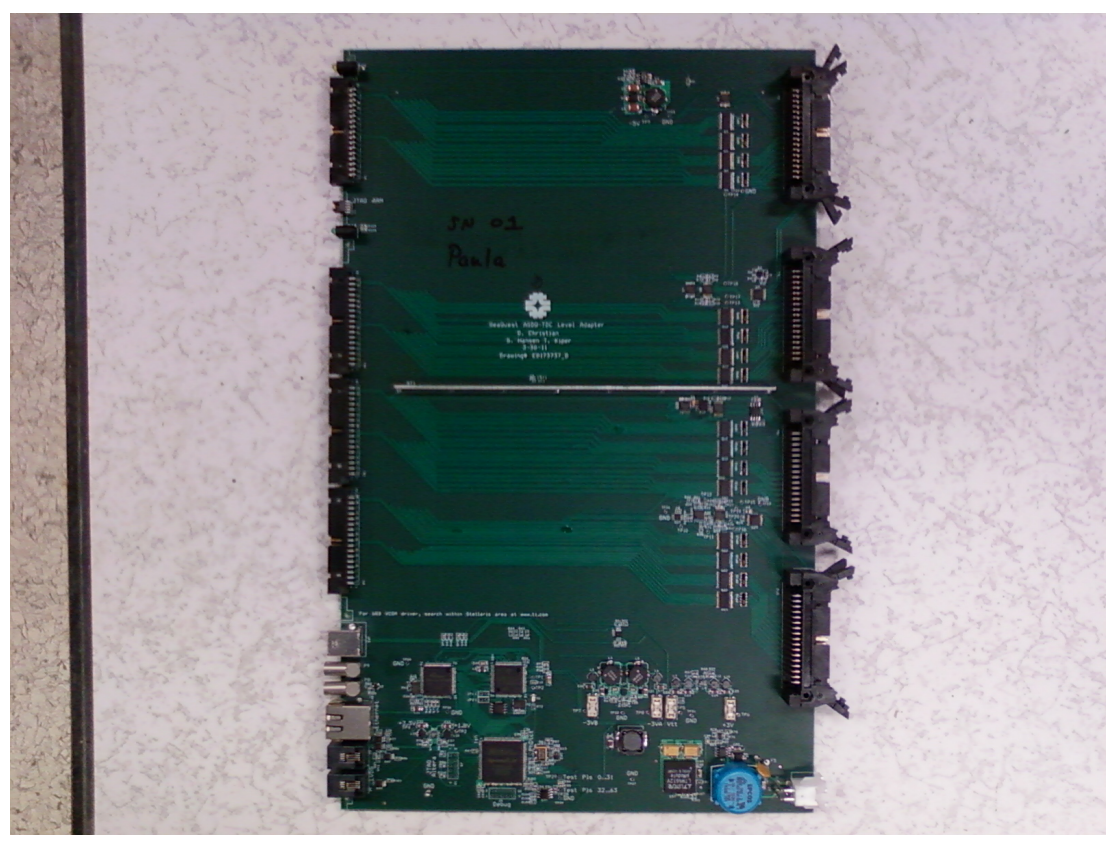

Figure 4.23: Picture of Level Shifter Board (LS board). LS board supplies low voltages to the ASDQ cards and converts the differential signals from the ASDQ cards to standard LVDS signals for TDC card. 8 ASDQ cards are connected to one LS board, and one LS board is connected to one TDC card.

signals from ASDQ cards go into the LS board, and the board converts the signals to standard LVDS signals. One LS board can operate 8 ASDQ cards, thus about 100 LS boards are in use in this experiment. The following features of the LS boards can be set by a standard telnet connection via an Ethernet cable:

- Threshold level adjustment: The threshold value in the ASDQ card can be set. The threshold is a 12 bit (0-4096) value that corresponds to 0-10 $\mathrm{mV}$ for amplified signal at BLR step in the ASDQ card.

- Internal test pulser: The LS board has an option to send a test pulse like a raw signal from drift chambers to ASDQ cards. This option helps to test the response of ASDQ cards.

- Voltage monitor: Voltages on several point of ASDQ card can be checked via the Ethernet connection. It is mainly for diagnostic purpose.

Figure 4.24 shows a chain of electronics. One ASDQ card has eight readout channels and each of which is connected to a sense wire of a drift chamber. Eight ASDQ cards are connected to one LS board. Thus, the number of input and output channels of one 


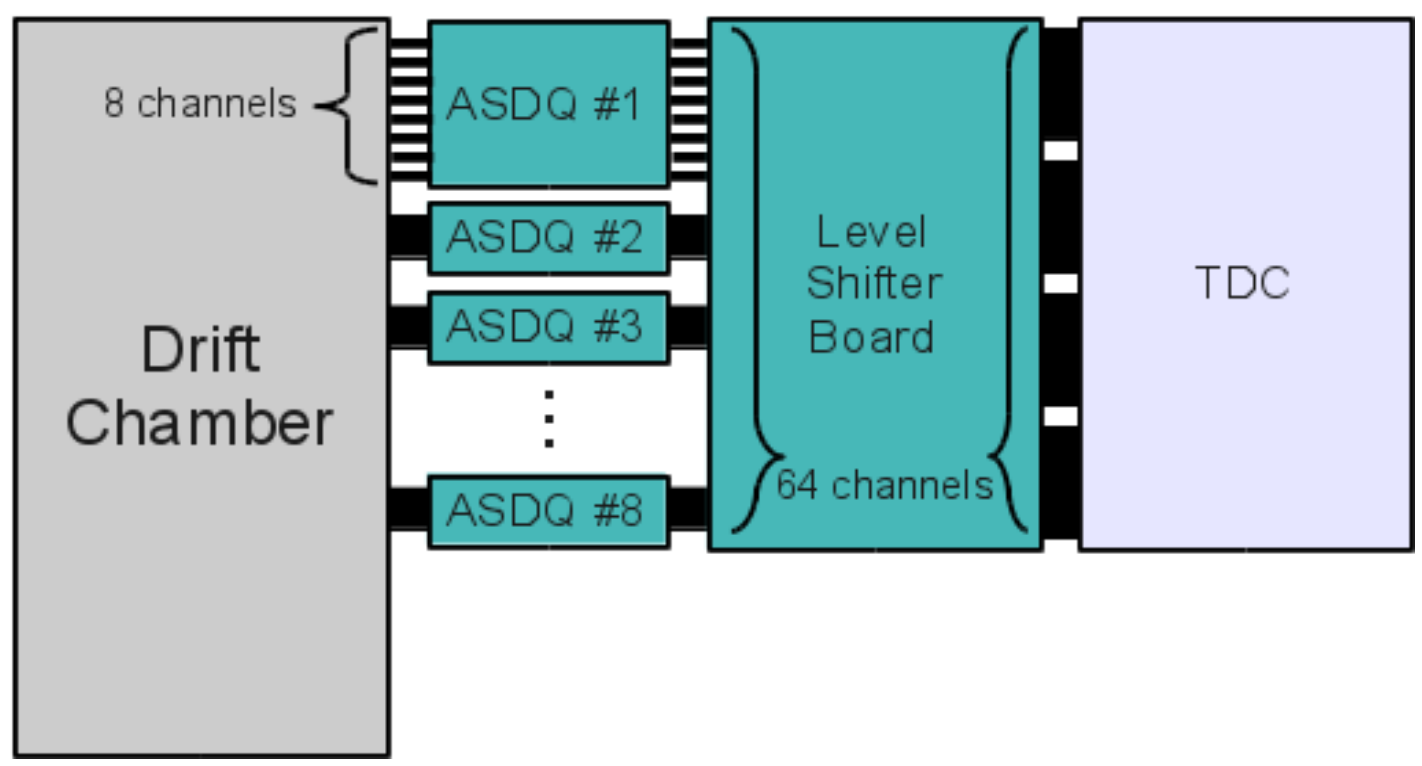

Figure 4.24: Chain of electronics. One ASDQ card has eight readout channels and each of which is connected to a sense wire of a drift chamber. Eight ASDQ cards are connected to one LS board. Thus, the number of input and output channels of one LS board is 64 channels. The TDC also has 64 channels, and one LS board is connected to one TDC.

LS board is 64 channels. The TDC also has 64 channels, and one LS board is connected to one TDC. 


\subsection{Monte Carlo simulation}

SeaQuest has two independent Monte Carlo simulations: "Fast Monte Carlo (FastMC)" and "Geant4 [32]-based Monte Carlo (GMC)". FastMC is a Monte Carlo simulation which was used in the E866/NuSea experiment and also used for the proposal of the SeaQuest experiment. We have developed GMC which is almost identical to FastMC except that it is a Geant4-based simulation so that we can deal with the magnetic field effect, muon penetration, detector response and so on in detail. GMC was used for all the simulation analyses shown in this thesis.

GMC first generates a dimuon with randomly chosen dimuon invariant mass, and Feynman- $x, x_{F}$. These two physics parameters are chosen in an uniform distribution of mass $(1$ to $10 \mathrm{GeV})$ and $x_{F}(-1$ to 1$)$ with kinematic cuts that ensure $P_{T}^{2}>0,0<x_{1}<1$, and $0<x_{2}<1$. Roughly $15 \%$ of events are cut off by these kinematic cuts. Geant4 is then used to track them through the spectrometer.

Once an event is created, an event weight is calculated. The weight is a function of the cross section of the physics process (Drell-Yan, $\mathrm{J} / \psi$ decay, $\psi^{\prime}$ decay). The Drell-Yan cross section is calculated by Eq. 2.9. Quark flavors used in the calculations are up, down, charm and strange. The cross section of $\mathrm{J} / \psi$ decay and that of $\psi^{\prime}$ decay are calculated by equations taken from [33]. The PDF parameterizations used in GMC is CTEQ6 [34]. 


\subsection{Detector acceptance}

Acceptance of the SeaQuest spectrometer for Drell-Yan events ( $\left.\mathcal{P}_{\text {detector }}\right)$ is evaluated using Monte Carlo simulation. It is defined as:

$$
\mathcal{P}_{\text {detector }} \equiv \frac{\# \text { of tracks in spectrometer acceptance }}{\# \text { of tracks in } 4 \pi}
$$

The detector acceptances as a function of mass, $x_{1}, x_{2}$, and $x_{2}$ vs $x_{1}$ are shown in Fig. 4.25. The $z$-axis of the 2D histogram indicates also the detector acceptance in percentage. A straight line of $x_{2}=x_{1}$ is drawn on the $2 \mathrm{D}$ histogram for reference.

The figure shows that the spectrometer focuses on high mass region as designed. Also, it focuses on high- $x_{1}$ and low- $x_{2}$. This is because that this experiment is a fixed-target experiment and detects dimuons going forward ( $+z$ direction). 

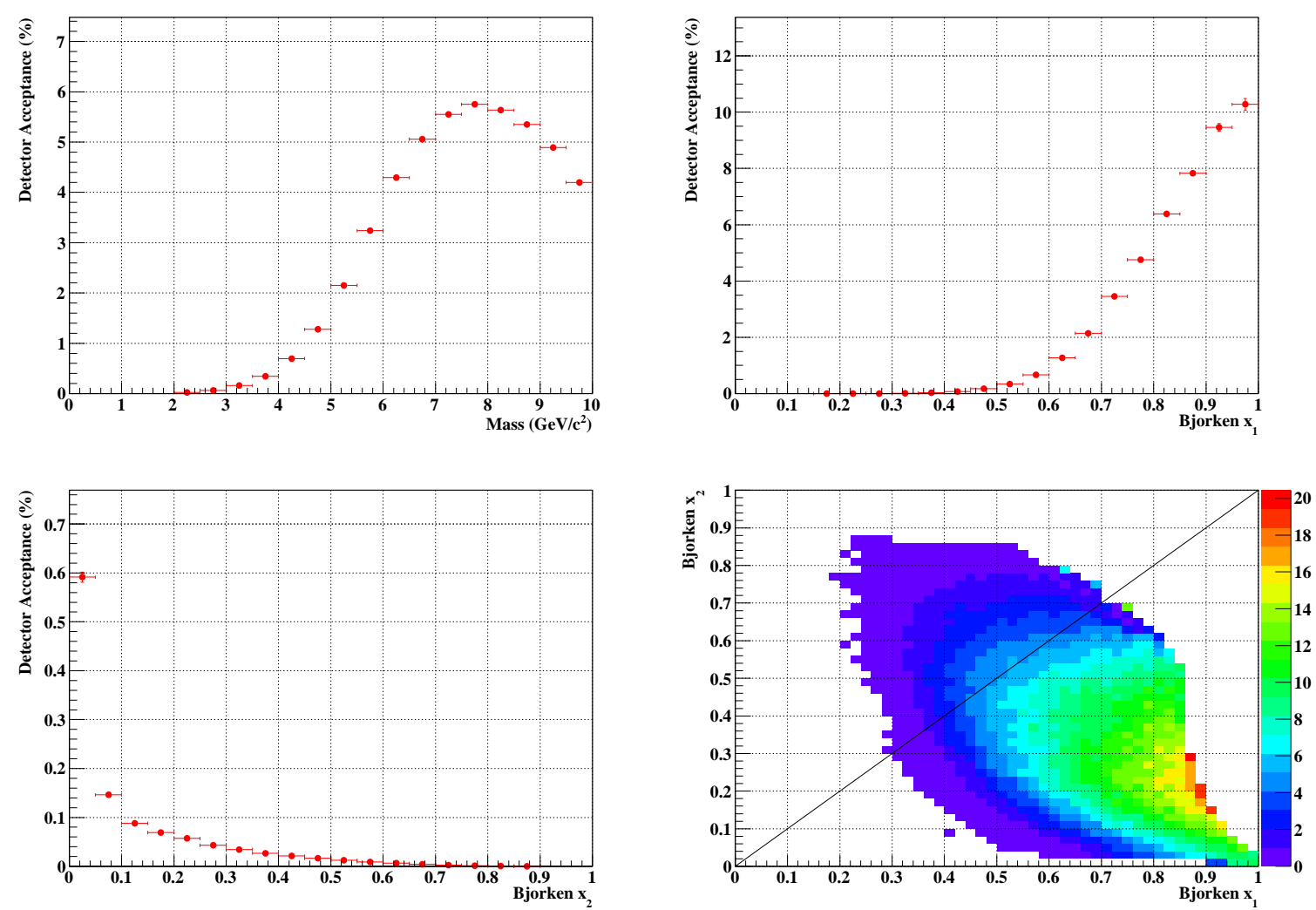

Figure 4.25: The detector acceptance as a function of mass, $x_{1}, x_{2}$, and $x_{2}$ vs $x_{1}$. The $z$-axis of the $2 \mathrm{D}$ histogram indicates also detector acceptance in percentage. A straight line of $x_{2}=x_{1}$ is drawn on the $2 \mathrm{D}$ histogram for reference. 


\subsection{Trigger}

SeaQuest trigger system uses the signals from the scintillator hodoscopes. It is optimized to accept high mass muon pairs produced in the target region. SeaQuest trigger system has two trigger types: FPGA-based trigger and NIM-based trigger. The data used in the analysis for this thesis were taken with FPGA-based trigger. The FPGA trigger system consists of five CAEN v1495 FPGA (Field Programmable Gate Arrays) VMEbus modules (see Fig. 4.26). On the other hand, the NIM trigger uses NIM modules to make a simpler trigger. Five different FPGA triggers and two different NIM triggers are used in SeaQuest physics run as listed in Table 4.10. They are called "FPGA-(ID\#)" or "NIM-(ID\#)". Each of the FPGA and NIM triggers is explained in the following subsections.

\begin{tabular}{lllllr}
\hline \hline Trigger type & \# of $\mu$ & Hodoscope side & Sign & Pt cut & Prescale factor \\
\hline FPGA-1 & dimuon & TB or BT & opposite & - & 1 \\
FPGA-2 & dimuon & TT or BB & opposite & - & 10000 \\
FPGA-3 & dimuon & TB or BT & same & - & 123 \\
FPGA-4 & single & T or B & any sign & - & 25461 \\
FPGA-5 & single & T or B & any sign & $>3$ GeV & 2427 \\
\hline NIM-1 & single & T or B & any sign & - & 31991 \\
NIM-3 & - & - & - & - & 125 \\
\hline
\end{tabular}

Table 4.10: Characteristics of the SeaQuest trigger types. Five FPGA triggers and two NIM triggers are used in physics run. "T" stands for top, and "B" stands for bottom. 


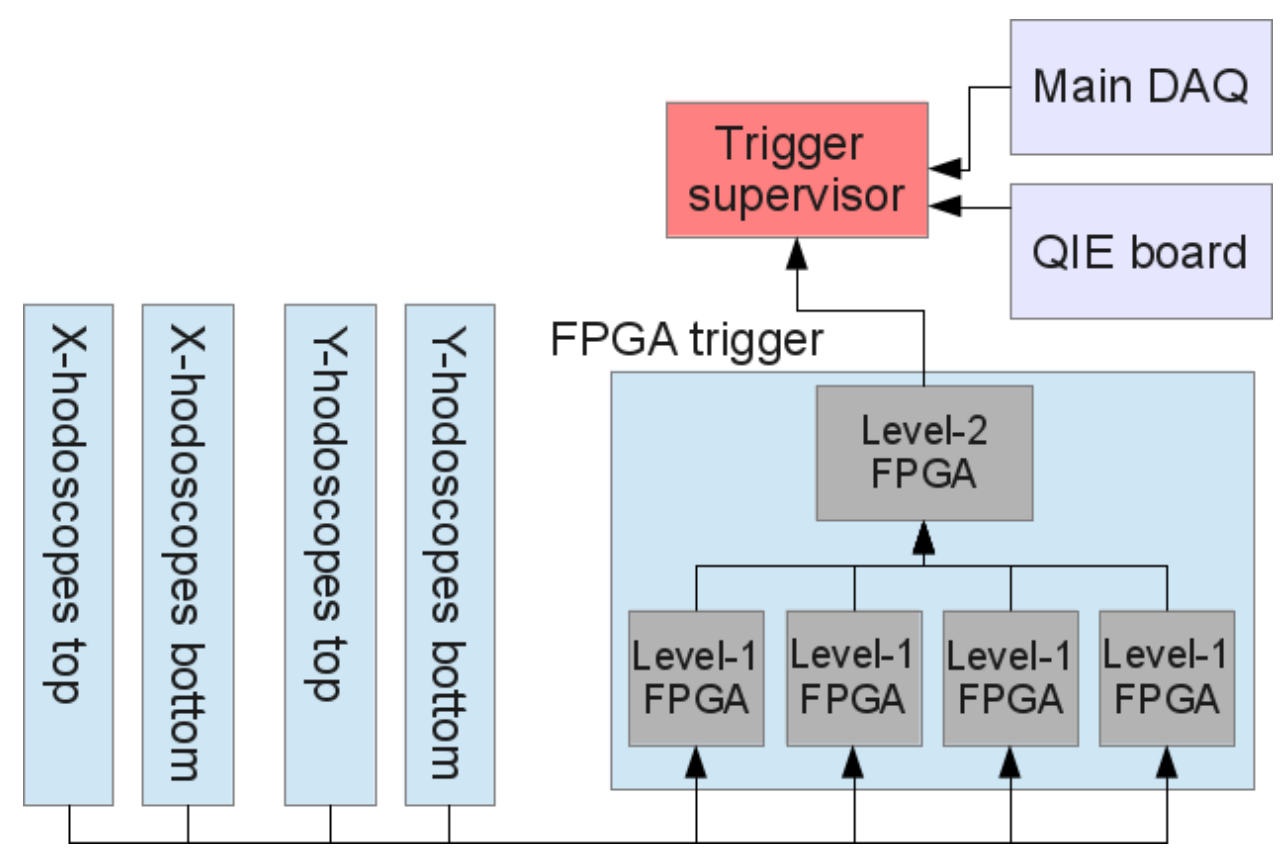

Figure 4.26: Overview of the FPGA trigger system of SeaQuest. It uses the signal from scintillator hodoscopes. It consists of a single decision stage, implemented as a two-step pipeline. They are called "Level-1", and "Level-2" trigger.

\subsubsection{FPGA trigger}

The FPGA trigger system consists of a single decision stage, implemented as a two-step pipeline. The first step is called "Level-1 trigger", and the second step is called "Level-2 trigger". The internal clock is synchronized with the RF clock provided from the Fermilab Accelerator division. Thus, the trigger is designed to output its decision per RF bucket.

In the first step, four different Level-1 triggers are used. Each of the four Level-1 triggers records the hit signal from either the $x-(y-)$ measuring hodoscopes in the top or bottom of the SeaQuest spectrometer. But, only two Level-1 trigger of the $x$-hodoscopes are used for the trigger during the physics run. Each Level-1 trigger logic identifies the hit combination of all the four hodoscope planes (called "trigger road"). For example, the Level-1 trigger for $x$-hodoscope in the top side identifies the hit pattern of H1TH2T-H3T-H4T. These patterns correspond to tracks of muon coming from target region. The FPGA trigger system has a list of selected hit patterns (look-up-table) in it. The look-up-table in the Level-1 trigger is especially called "Roadset", and this defines the kinematic acceptance of this experiment. Roadsets are optimized for high mass dimuons, based on Monte Carlo simulations first. They are subsequently fine-tuned by hand in order to achieve high efficiency for triggering high mass dimuons with excluding low mass 


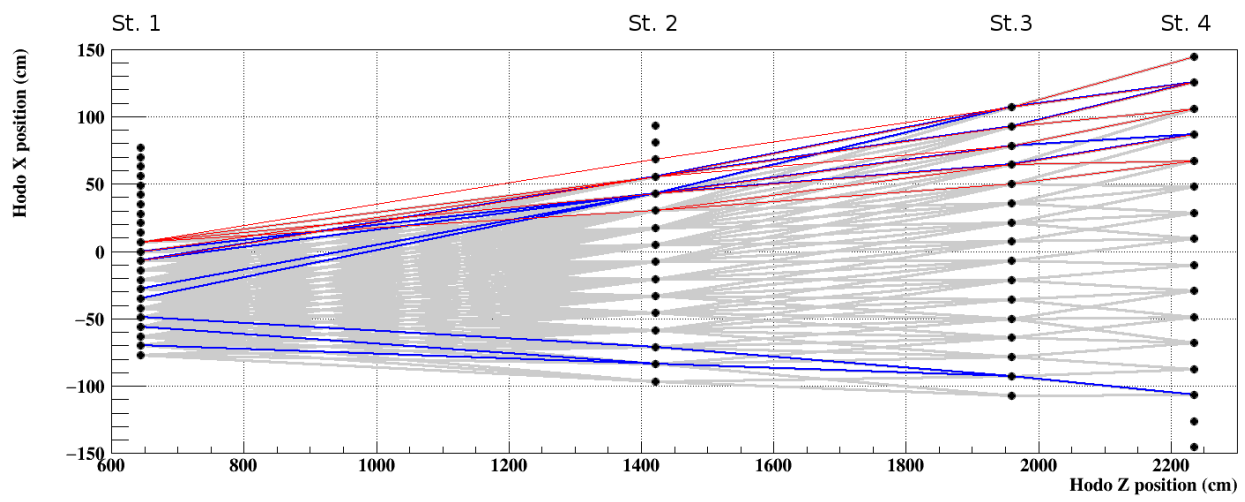

Figure 4.27: Visualization of the hit combination of negative muon on the $x$-hodoscopes. The black points represent scintillator paddles viewed from the top. The red line shows the 10 most-frequently hit combinations, and the blue lines show the next 10 . Other combinations are shown in gray.

dimuons. Roadset is usually named with number like "Roadset 57", and the number is varied when the set is modified even if the change is small. After the Level-1 trigger checked the recorded hit pattern, it sends its result to Level-2 trigger.

Level-2 trigger combines the Level-1 trigger's results. There is also a look-up-table for the combination in the Level-2 trigger system to identify events with candidate high mass muon pairs. The candidate muon pairs are characterized according to their combination of charge, the side of the spectrometer on which they are recorded, and a rough measure of their $P_{T x}$ (transverse momentum). There are five different look-up-tables based on physics purpose: FPGA1-5 (see Table 4.10 ).

FPGA-1 trigger is the primary physics trigger for physics analysis. It selects oppositelycharged muons that traversed opposite sides of the hodoscopes (top and bottom) while FPGA-2 trigger selects oppositely-charged muons that traversed the same sides of the hodoscopes (top-top or bottom-bottom). FPGA-3 trigger selects the same sign muons that traversed opposite sides of the hodoscopes.

FPGA-4 and FPGA-5 trigger on single muon track that traversed either on the topside or bottom-side of the detector. Only FPGA-5 trigger has a $P_{T x}$ cut. The trigger aims to select muons that have higher $P_{T x}$. Those single muon triggers are used to extract combinatorial backgrounds.

\subsubsection{NIM trigger}

When the FPGA triggers were under development, NIM-based trigger was used for the SeaQuest trigger system. NIM-1 trigger is a coincidence of H1T-H2T-H3T-H4T or H1B- 
H2B-H3B-H4B. NIM-3 trigger is a special random trigger. It is a coincidence of RF signal sent from the Fermilab accelerator division and $7.5 \mathrm{kHz}$ pulse signal produced by a NIM gate generator. It randomly picks up an RF bucket and is aimed to study the background rate.

\subsubsection{Roadset in physics run}

This thesis shows results using the data taken by three different Roadsets of FPGA-based trigger: "Roadset 57", "Roadset 62", and "Roadset 67". The Roadset 57 is the first successful trigger set for physics analysis. It focuses on high mass dimuons as designed. The Roadset 62 is mostly the same set with Roadset 57, but containing a few roads for dark photon search produced at downstream of the FMAG and penetrating into the spectrometer acceptance. The Roadset 67 is made by symmetrizing the Roadset 62 and omitting some hot roads. Hot road is a road that is anomalously fired due to noisy hodoscope planes or massive low mass muons. Trigger probability $\left(\mathcal{P}_{\text {trigger }}\right)$ is defined as follows in order to know how much the muon tracks are triggered by the trigger:

$$
\mathcal{P}_{\text {trigger }} \equiv \frac{\# \text { of tracks accepted by FPGA1 trigger }}{\# \text { of tracks in spectrometer acceptance }}
$$

This is calculated using Monte Carlo simulations. The trigger probability of these three Roadsets as a function of mass, $x_{1}, x_{2}$ are shown in Fig. 428. The figure shows that the FPGA1 trigger focuses on high mass dimuons $(M \sim 6 \mathrm{GeV})$, and defocuses low mass dimuons including dimuons from $J / \psi$ decay in order to achieve high efficiency for detecting dimuons from Drell-Yan process. The trigger covers high Bjorken $x_{2}$ area as designed. The trigger probability of Roadset 57 and 62 are identical. The probability of the Roadset 67 is $10 \%$ larger than the others because it has $10 \%$ more roads than the others.

\begin{tabular}{lll}
\hline \hline Roadset & \# of roads & Description \\
\hline 57 & 871 & The first successful trigger set for physics analysis. \\
\hline 62 & 891 & $\begin{array}{l}\text { Mostly the same set with Roadset } 57 \text { but containing few } \\
\text { roads for dark photon originating at behind the FMAG. }\end{array}$ \\
\hline 67 & 977 & $\begin{array}{l}\text { Made by symmetrizing the Roadset } 62 \text { and omitting } \\
\text { some hot roads. }\end{array}$ \\
\hline
\end{tabular}

Table 4.11: Summary of the Roadsets 57, 62, and 67. 

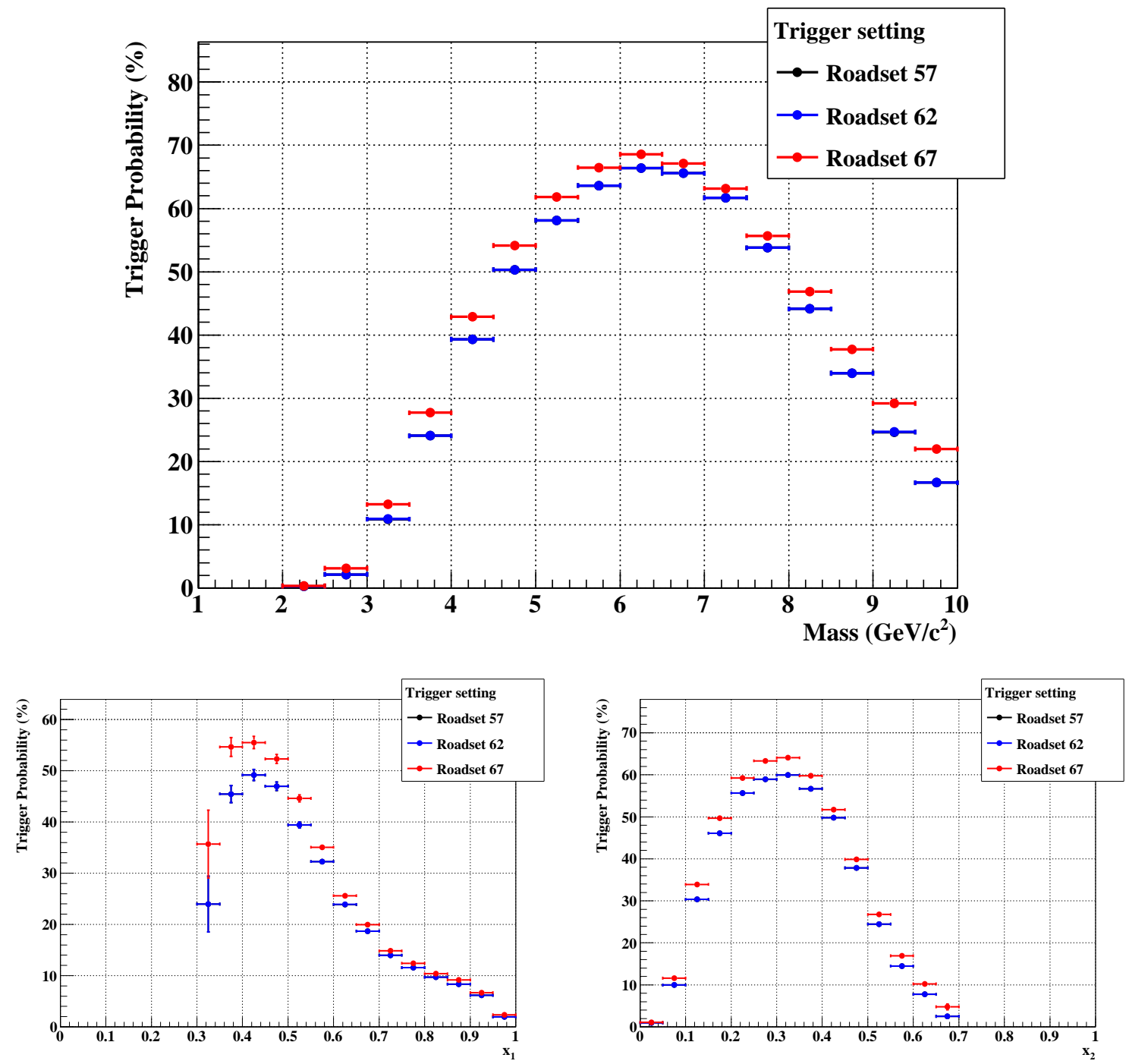

Figure 4.28: Trigger acceptance of Roadset 57, 62, and 67 as a function of dimuon invariant mass, Bjorken $x_{1}$, and $x_{2}$. The trigger probability of Roadset 57 and 62 are identical and are shown in blue. The probability of the Roadset 67 is $\sim 10 \%$ larger than the others because it has $\sim 10 \%$ more roads than the others. The FPGA1 trigger focuses on high mass dimuons $(M \sim 6 \mathrm{GeV})$ and defocuses on low mass dimuons including dimuons from $J / \psi$ decay. The trigger covers high Bjorken $x_{2}$ area as designed. 


\subsection{Data Acquisition System}

SeaQuest has three data acquisition (DAQ) systems called "Main DAQ", "Scaler DAQ", and "Beam DAQ". Main DAQ records the main detector information and the trigger timing. Scaler DAQ records the scaler information once per spill. Beam DAQ records information from the Cherenkov monitor which is read out by QIE board at the RF frequency.

\subsubsection{Main DAQ}

The Main DAQ is driven by the CODA (CEBAF On-line Data Acquisition) [35] system developed by the Jefferson Lab Data Acquisition Group. CODA is a kit of parts that allows us to implement a data acquisition system. The most common format for electronics is VME. The Main DAQ consists of 14 VME crates as shown in Fig. 4.2. One of the 14 crates is a special crate that holds Trigger Supervisor (TS) drawn in the most left hand side in the figure. The function of the TS is to accept the trigger from either the FPGA Level-2 or the NIM trigger, and fan it out toward the other 13 VME crates. Each VME crate has a VME CPU, a Trigger Interface Card (TIR), and a set of TDCs for hodoscopes, drift chambers, and trigger systems. The VME CPU called ROC (ReadOut Controller) is the central processor of the crate that reads out the TDCs when TIR receives a trigger signal. Figure. 4.30 shows an example of the VME crates.

The TS has 12 trigger slots and each can accept trigger signal at a time. For the SeaQuest experiment, the first 5 triggers are NIM-based (two of them are usually in use as described in the trigger section), the second 5 are from the FPGA Level-2, and the last two are the beginning of spill (BOS) and the end of spill (EOS) signals that come from the Fermilab Accelerator Division.

Once the event is accepted by the trigger logic, the beam quality around the triggered RF bucket is checked before the trigger signal goes to TS. Cherenkov counter and its readout system, QIE, measures intensity of each RF bucket as described before. If the intensity of an RF bucket is higher than the user-selected threshold, the QIE issues a veto signal for $+/-16 \mathrm{RF}$ buckets around the RF bucket. When the veto signal is high, trigger signals are blocked.

Following two items should be satisfied for the trigger signal to be accepted by TS:

- DAQ is not reading data.

- Trigger is not "Latched" in the trigger slot. 


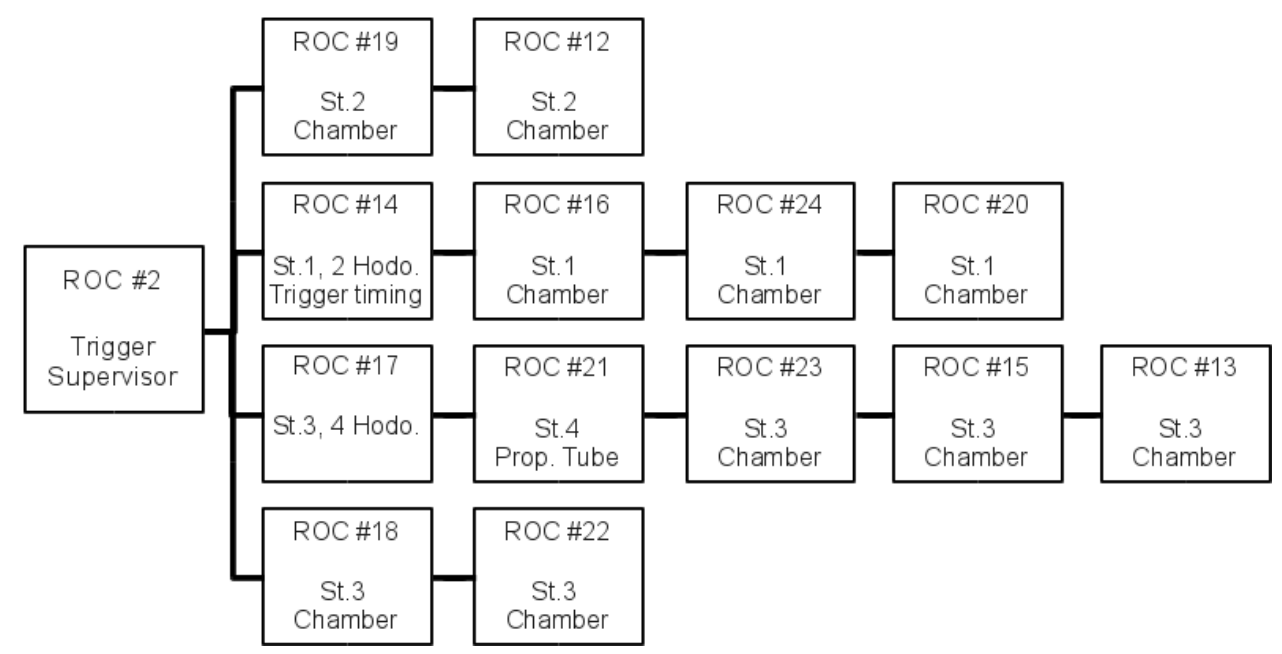

Figure 4.29: A chain of VME crates of the Main DAQ. It consists of 14 VME crates. The crate in the most left hand side is the special crate that holds Trigger Supervisor (TS). The TS accepts trigger, and fan it out toward the other 13 VME crates.

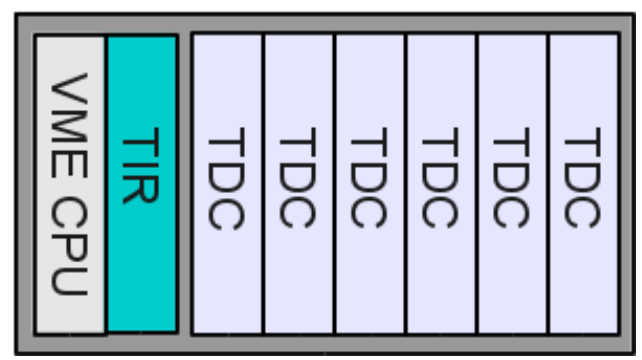

Figure 4.30: Example of the VME crates. Each VME crate has a VME CPU, a Trigger Interface Card (TIR), and a set of TDCs for hodoscopes, drift chambers, and trigger systems. 
where "Latched" means that trigger signal is already accepted in the trigger slot for the RF. Therefore, the first trigger signal is accepted for each trigger type when TS is not reading data (the status is called "Live"), and other trigger signals are blocked during that RF clock period.

Not to disturb the main trigger (FPGA-1 trigger), the other trigger types are scaled down by a factor of $i$ : only every $i$-th triggered event is accepted for each trigger. The typical number of the factors for the triggers are listed in Table. 4.10. Those numbers are set so that the trigger rate, except FPGA-1 trigger, becomes less than $10 \%$ of the FPGA-1 trigger rate. If the trigger signal passed the condition coming from scaling down, the triggered event is finally accepted and the DAQ starts recording the data. The flow of data is following:

1. TS receives trigger from NIM or FPGA Level-2 or both, if there is no beam veto signal.

2. TS outputs trigger to the 13 TIRs once it accepted the trigger.

3. The TDCs record time when the trigger signal arrives from TIR.

4. After TDCs stop, ROC starts reading the TDCs, and tells TIR that it has finished reading.

5. The TIR sends an acknowledge back to the TS saying that the particular ROC finished reading all of its TDCs.

6. The TS, once it receives an acknowledge from all 13 VME TIRs, is ready to receive the next trigger.

Usually one "run" corresponds to the data taking of about 60 minutes (which mostly equals to 60 spills). The typical trigger rate of one spill is roughly 5000 to 10000 depending on the run settings and conditions. The typical data size of one run is $\sim 1$ GB.

\subsubsection{Scaler DAQ}

The Scaler DAQ is a standalone DAQ that is designed to monitor the quality of the beam coming into SeaQuest. It is also driven by CODA system. There are four VME scalers installed on the Scaler DAQ. One of them is triggered by the coincidence of $7.5 \mathrm{kHz}$ trigger by gate generator and the RF signal. This records hit counts of hodoscope planes at $7.5 \mathrm{kHz}$. The other three scalers are triggered by the BOS or EOS signals and thus record spill-level rates. Data collected by these spill-level scalers are the number of times 
each Main DAQ trigger is satisfied, intensity of the beam, and the rates of the hodoscope arrays. The data from the Scaler DAQ is fed back to the Accelerator Division to monitor and tune the beam.

\subsubsection{Beam DAQ}

The Beam DAQ is responsible for recording the $53 \mathrm{MHz}$ structure of the beam, namely the intensity of each RF bucket. The core of the Beam DAQ is the QIE board. There are three types of data that are recorded by the QIE board during the spill:

- Intensity of each RF bucket

- Number of protons inhibited due to high instantaneous intensity

- Number of protons missed because the Main DAQ was busy. This number excludes inhibited protons to avoid double counting.

Those data are output to ASCII files.

\subsection{Decoder and production}

The three raw outputs of DAQs are Main DAQ CODA files, Scaler DAQ CODA files, and Beam DAQ ASCII files. These three types require varying degrees of de-serialization, parsing, processing, and storage. This process is defined as "decoding". All raw data files are backed up to tape storage (managed by Fermilab Computing Division). The decoded and processed data are stored on MySQL servers to be used for analysis. The MySQL is an open-source Relational Database Management System (RDBMS) developed by Oracle that is well suited for the storage and responsive querying of hierarchical data. Each run is decoded into its own schema, and contains its own instances of all tables of a specified user-friendly design for analysis.

\subsection{Fabrication of Station 3 minus drift chamber}

The Station 3 minus drift chamber was newly fabricated by SeaQuest collaborator in Fermilab under the initiative of the Japanese group. Table. 4.2 shows the schedule of the fabrication. 


\begin{tabular}{ll}
\hline \hline Term & Work list \\
\hline Jun., 2012 & • Assemble chamber frame \\
& • Clean chamber frame \\
& - Put feed-throughs with adhesive \\
\hline Jul.-Oct., 2012 & • Assemble wires \\
& - Tension measurement \\
\hline
\end{tabular}

Nov., 2012 - Jan., 2013

- Fabricate chamber windows

- Attach chamber windows

- Attach gas connectors

Feb.-Apr., 2013

- Fabricate and attach electronics (readout and HV parts)

- Start flowing gas

May-Jul., 2013

- Repair broken wires

- HV training

Aug-Sep., 2013

- Install into the SeaQuest spectrometer

- Connect readout electronics, HV cables and gas lines

Table 4.12: Schedule of the fabrication of the Station 3 minus drift chamber at Fermilab. 


\subsubsection{Wire assembling}

The most important and sensitive work in the fabrication of the drift chamber is assembling wires. A total of 16 people including myself worked to assemble 5154 wires (768 sense wires and 4386 cathode wires). Following is the procedure of the wire assembling with two persons (see Fig. 4.3):

1. A worker at the right side puts a wire into the feed-through, and passes the wire to the other side.

2. Once the left worker received the wire, the right worker solders the wire to feedthrough. An appropriate amount of solder is required. If it is too less, the wire can be dropped, and if it is too much, readout connectors cannot be adapted.

3. After soldering the right feed-through is done, the left worker carefully hangs the weight on the wire to apply tension to the wire, then solders it to feed-through.

4. Cut extra unused wire, and prepare for next wire.

The tensions to the sense wires and cathode wires are 85 gf and 130 gf, respectively. These values are determined by taking into account the effect of electrostatic forces between wires and the sag of the chamber frame.

It takes roughly four minutes to assemble one sense wire while it takes roughly two minutes to assemble one cathode wire. This difference is caused by the sense wire's thinner diameter, which makes the wiring work more difficult.

\subsubsection{Tension measurement}

Measuring the tension of wires in drift chambers after the construction is an important process because sometimes wires get loose after the soldering. The wire tension was measured by detecting the wire vibration caused by external Lorentz force. A wire, a function generator, an oscilloscope, and a low-pass filter are connected as shown in Fig. 4.32. Also, a permanent magnet is placed close to the wire to apply magnetic force vertically to the wire.

Pulse signal from the function generator produces a periodic Lorentz force, which makes the wire vibration. If the frequency of the periodic force is close to the resonant frequency of the wire, the wire widely vibrates. The magnitude of the vibration depends on the frequency of the periodic force, pulse height, pulse length, and strength of the magnetic force. Induced current produced by the vibration across the magnetic field is 


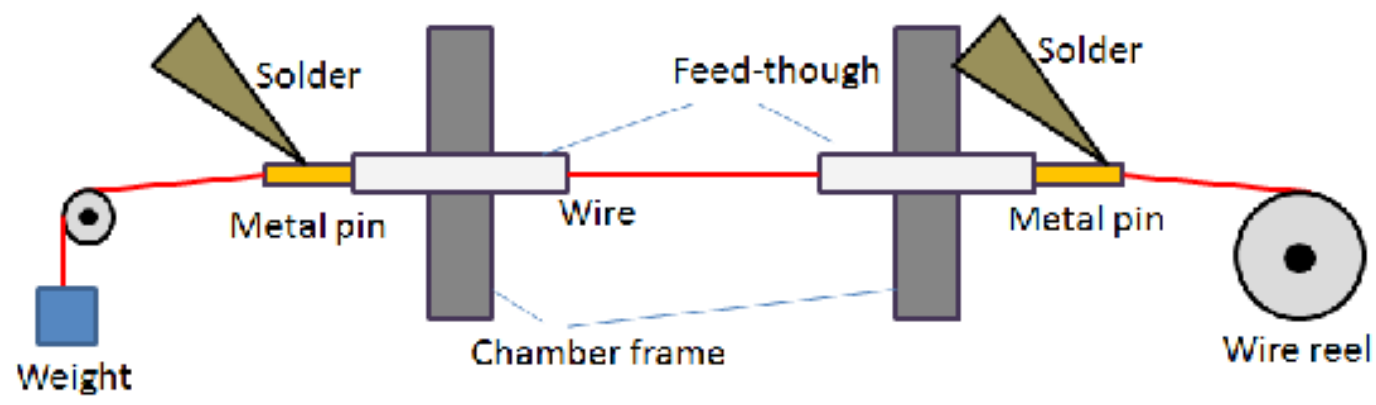

Figure 4.31: Overview of the wire assembling (side view). This work is done by two persons; one works on the right side, and the other works on the left side.

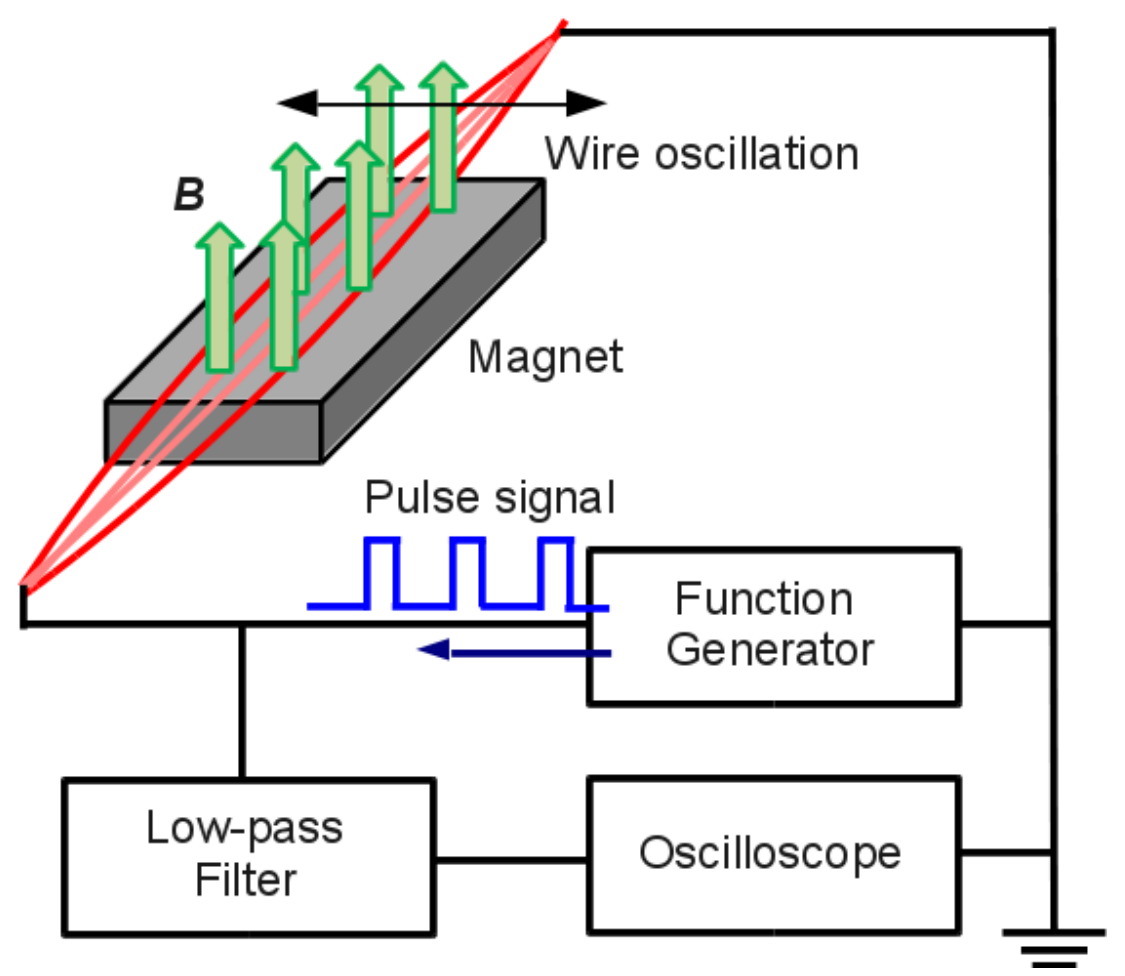

Figure 4.32: Overview of the tension measurement. The external force due to the pulse wave whose frequency is set at $f_{\text {eigen }} / 3$ makes the wire vibration. The wire vibration in the magnetic field produces an induced current which is observed by oscilloscope. The low-pass filter before the oscilloscope helps to remove high-frequency noise. 


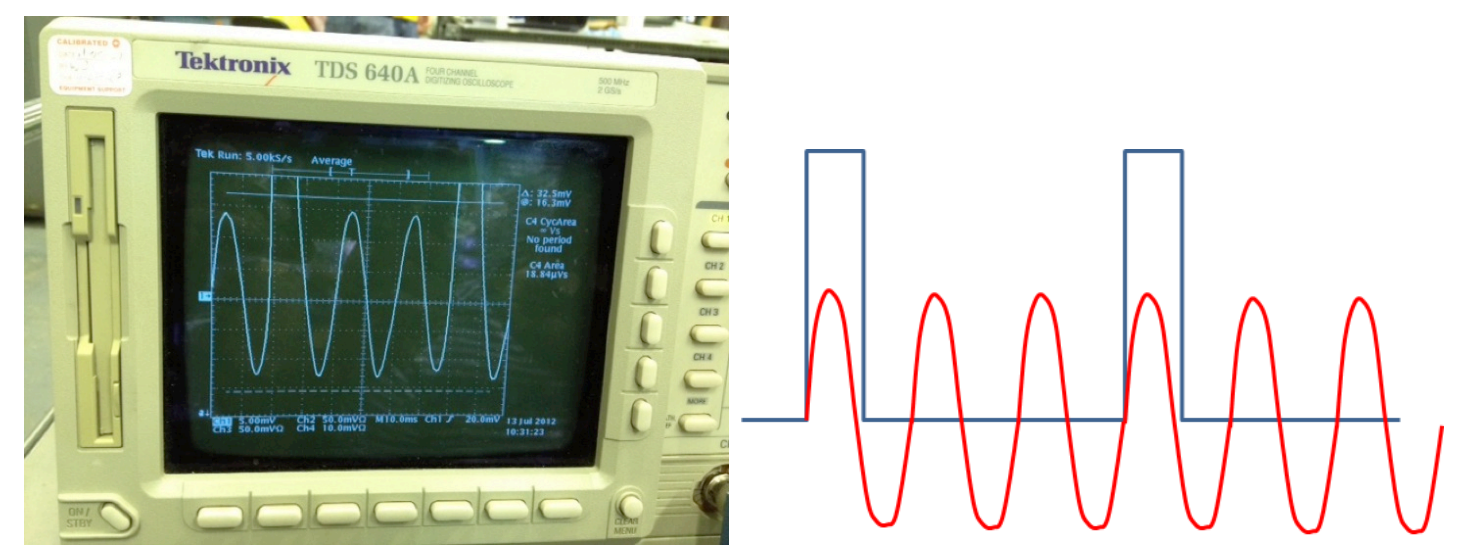

Figure 4.33: Left photo shows the sinusoidal wave of the induced current with pulse of $f_{\text {eigen }} / 3$ displayed on the oscilloscope. Right figure expresses the wave form; pulse wave (blue) and induced current wave (red). The wave is clearly seen due to the lowpass filter. A person finds frequency where the amplitude of the induced current wave becomes maximum. Then, three times of the measured frequency is the eigen frequency of the wire. Tension of the wire is calculated by Eq. 4.3.

observed by the oscilloscope. In the measurement, pulses of $f_{\text {eigen }} / 3$ were sent to the wire not to disturb to display the wave of the induced current by the pulse. A person scans frequency around $f_{\text {eigen }} / 3$, and finds frequency where the amplitude of the induced current wave becomes maximum. The left photo of Fig. 4.33 shows the oscilloscope display during the tension measurement. It shows the sinusoidal wave of the induced current together with pulses of $f_{\text {eigen }} / 3$. Then, three times of the measured frequency is the eigen frequency of the wire. The tension of the wire is calculated by

$$
f=\frac{n}{2 L} \sqrt{\frac{T}{\sigma}}, \quad n=1,2,3, \cdots
$$

where $f$ is the resonant frequency of the wire, $L$ the length of the wire, $T$ the tension of the wire, and $\sigma$ line density of the wire.

Figure 4.34 shows some of results of tension measurements. The left figure shows a histogram of tensions of sense wires at 5th plane. The mean value is $84.2 \mathrm{gf}$, and the standard deviation is $4.01 \mathrm{gf}$ The right figure shows that of cathode wires at 6th plane. The mean value is $128.6 \mathrm{gf}$, and the standard deviation is $1.62 \mathrm{gf}[36]$ The wires were stretched with high accuracy. The wires of the other planes were also stretched with high accuracy. All the results are found in [36] . 

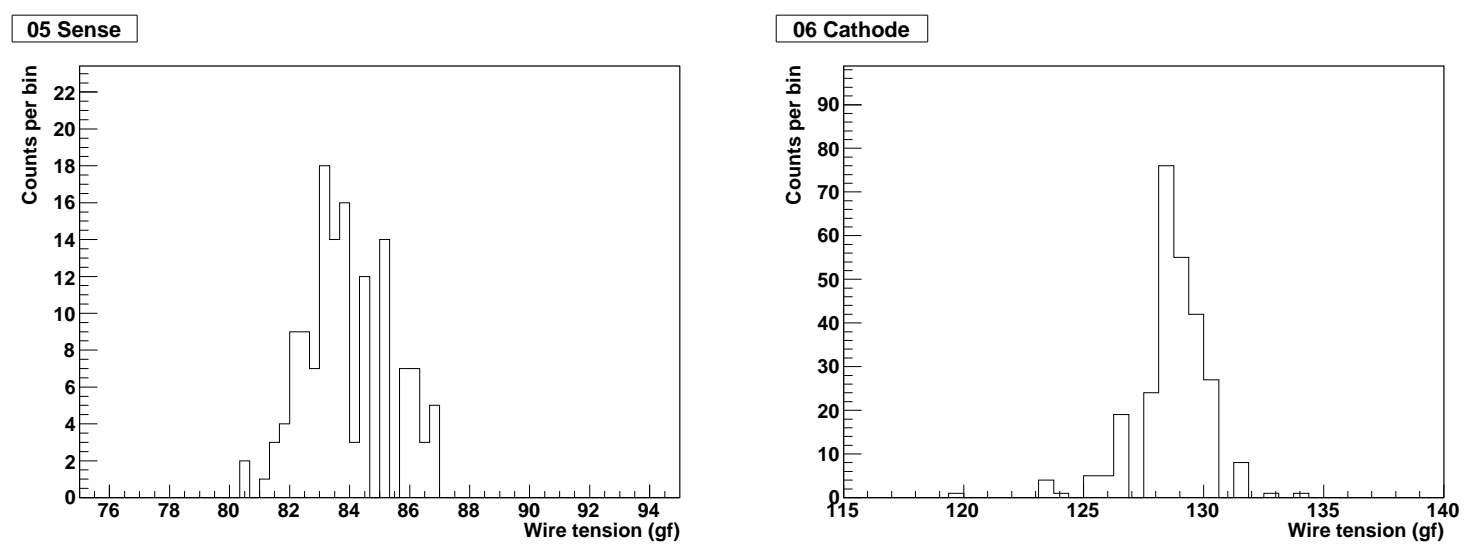

Figure 4.34: The left figure shows a histogram of tensions of sense wires at 5th plane. The right figure shows that of cathode wires at 9th plane [36]. 


\section{Chapter 5}

\section{Data Analysis}

\subsection{Data sets}

The experimental data recorded by DAQ are categorized according to trigger road set (called "Roadset" with a number) used for data taking as shown in Table 5 . The data taken by "Roadset 57 ", "Roadset 62 " and "Roadset 67 " are analyzed in this thesis. The Roadset 57 data is the first data for physics analysis in the SeaQuest experiment. It was taken during the run II. The Roadset 62 and Roadset 67 data are the second- and the third Roadset data for the physics analysis. They were taken during the run III.

\begin{tabular}{llllrr}
\hline \hline Run & Roadset & Period & Run number & \# of runs & \# of spills \\
\hline II & 57 & $2014.06 .25-2014.08 .20$ & $8912-10420$ & 1305 & 61560 \\
II & 62 & $2014.11 .08-2015.01 .14$ & $11075-12438$ & 1254 & 85928 \\
III & 67 & $2015.01 .25-2015.06 .19$ & $12525-15789$ & 3097 & 182568 \\
\hline
\end{tabular}

Table 5.1: Summary of the Roadset data. These three Roadset data are analyzed in this thesis.

\subsection{Spill Selection}

\subsubsection{Applied spill cuts}

The first cut applied to the data sets is a spill-level cut listed in Table 5.2. The duration of a spill is 5 seconds in every one minute as described in the previous chapter. The spill-level cut means spill-by-spill selection. The quantity used in the cut are summarized in Table 5.3.

The SeaQuest targets are numbered from 1 to 7 (see Table. 4.2). If the target number is incorrect, such a spill cannot be used for analysis. "AfterInhFPGA1" and "Accept- 


\begin{tabular}{lll}
\hline \hline Quantity & Roadset 57 & Roadset $62 \& 67$ \\
\hline Target position & {$[1,7]$} & {$[1,7]$} \\
AfterInhFPGA1 & {$[1000,30000]$} & {$[100,10000]$} \\
AcceptedFPGA1 & {$[1000,8000]$} & {$[100,6000]$} \\
AcceptedFPGA1/AfterInfFPGA1 & {$[0.2,0.9]$} & {$[0.2,1.05]$} \\
TSGo & {$[1000,8000]$} & {$[100,6000]$} \\
G2SEM & {$[2 \mathrm{e} 12,1 \mathrm{e} 13]$} & {$[2 \mathrm{e} 12,1 \mathrm{e} 13]$} \\
QIEsum & {$[4 \mathrm{e} 10,1 \mathrm{e} 12]$} & {$[4 \mathrm{e} 10,1 \mathrm{e} 12]$} \\
Inhibit & {$[4 \mathrm{e} 9,1 \mathrm{e} 11]$} & {$[4 \mathrm{e} 9,2 \mathrm{e} 11]$} \\
Busy & {$[4 \mathrm{e} 9,1 \mathrm{e} 11]$} & {$[4 \mathrm{e} 9,1 \mathrm{e} 11]$} \\
Duty factor & {$[15,60]$} & {$[10,60]$} \\
\hline
\end{tabular}

Table 5.2: Summary of cuts for a spill. The cuts for Roadset 57 data and those for Roadset $67 \& 67$ data are slightly different due to different run conditions.

edFPGA1" are the numbers of events counted during the data taking process as shown in the flow chart of Fig. 5. In order to know the efficiency of data taking. "TSGo" is the total number of accepted events fired by any trigger. The TSGo rate is mostly equal to the AccepedFPGA1 rate because the events by other triggers are scaled down (the scale factors are listed in Table 4.10). "G2SEM" and "QIEsum" are the total intensity of the proton beam in the spill measured by G2SEM or Cherenkov counter. "Inhibit" is the number of RF buckets blocked by QIE VETO, and "Busy" is the number of RF buckets blocked by TS busy. How the RF status is labeled is shown in Fig. 5.2.

"Duty factor" tells us how stable the intensity is. The definition of the duty factor is $\langle I\rangle^{2} /<I^{2}>$, where $I$ is the number of protons in one RF bucket. The cuts for Roadset 57 data and those for Roadset $67 \& 67$ data are slightly different due to different run conditions. The quantities of these Roadset data are shown as a function of spill ID (in other words, time dependence of the quantity) in Fig.5.3-5.7. The spill whose all quantities are in the good range is regarded as "good spill", and is shown in red. If any of the quantities is out of the range, the spill is regarded as "bad spill", and is shown in black in all the figures. 


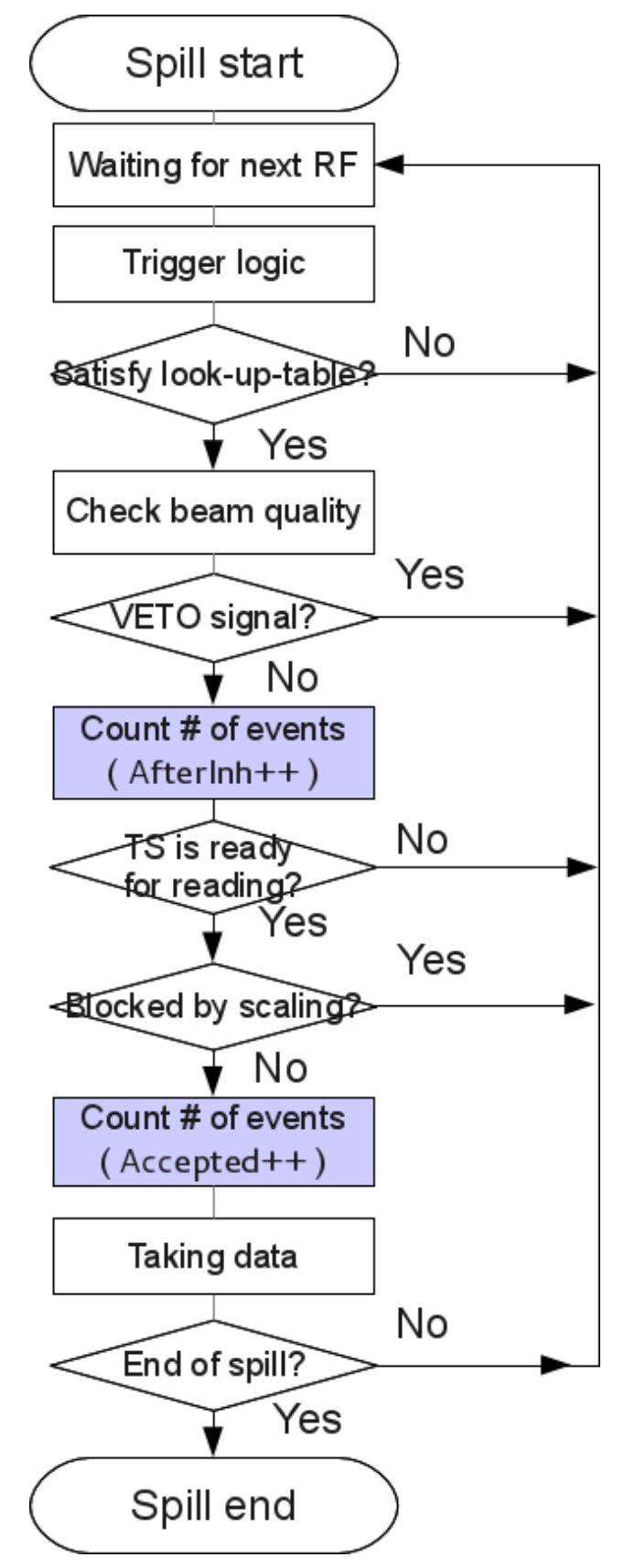

Figure 5.1: Flow chart of data taking in one spill. "AfterInh" is the number of events that passed the beam quality check. "Accepted" is the number of events accepted by Trigger Supervisor to start taking data. 


\begin{tabular}{|c|c|}
\hline Quantity & Description \\
\hline Target position & $\begin{array}{l}\text { Target position numbered from } 1 \text { to } 7 \text {, as listed in Ta- } \\
\text { ble. ‥2. }\end{array}$ \\
\hline AfterInhFPGA1 & $\begin{array}{l}\text { Number of events which are not inhibited (FPGA1) (see } \\
\text { Fig. [-1]) }\end{array}$ \\
\hline AcceptedFPGA1 & $\begin{array}{l}\text { Number of events which are accepted (FPGA1) (see } \\
\text { Fig. [ل. }\end{array}$ \\
\hline TSGo & $\begin{array}{l}\text { Number of accepted events fired by any trigger (namely, } \\
\text { AcceptedFPGA1 } \cup \text { AcceptedFPGA2 } \cup \cdots \cup \text { Accepted- } \\
\text { NIM3) }\end{array}$ \\
\hline G2SEM & Total intensity measured by G2SEM in the spill \\
\hline QIEsum & $\begin{array}{l}\text { Total intensity measured by Cherenkov counter in the } \\
\text { spill }\end{array}$ \\
\hline Inhibit & $\begin{array}{l}\text { Number of RF buckets blocked by QIE VETO (see } \\
\text { Fig. (5.2) }\end{array}$ \\
\hline Busy & Number of RF buckets blocked by TS busy (see Fig. $5 \cdot 2$ ) \\
\hline Duty factor & Duty factor at $53 \mathrm{MHz}$ \\
\hline
\end{tabular}

Table 5.3: Description of the quantities for spill cuts. The quantities are recorded for each spill.

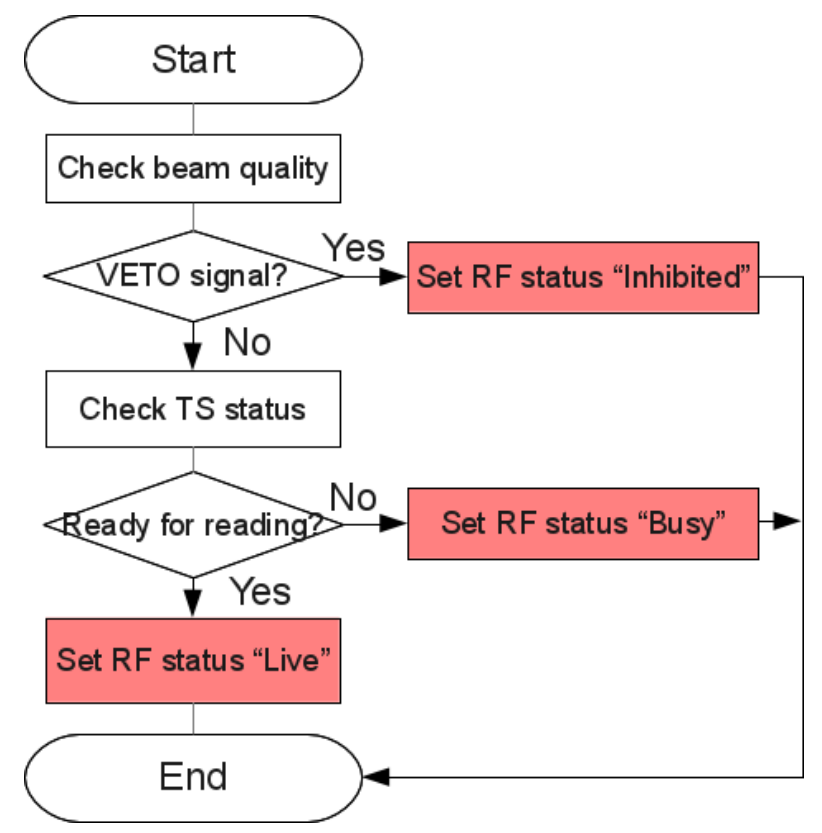

Figure 5.2: Flow chart of defining the status of RF bucket. The RF buckets are categorized into these three status: "Inhibited", "Busy" or "Live". 

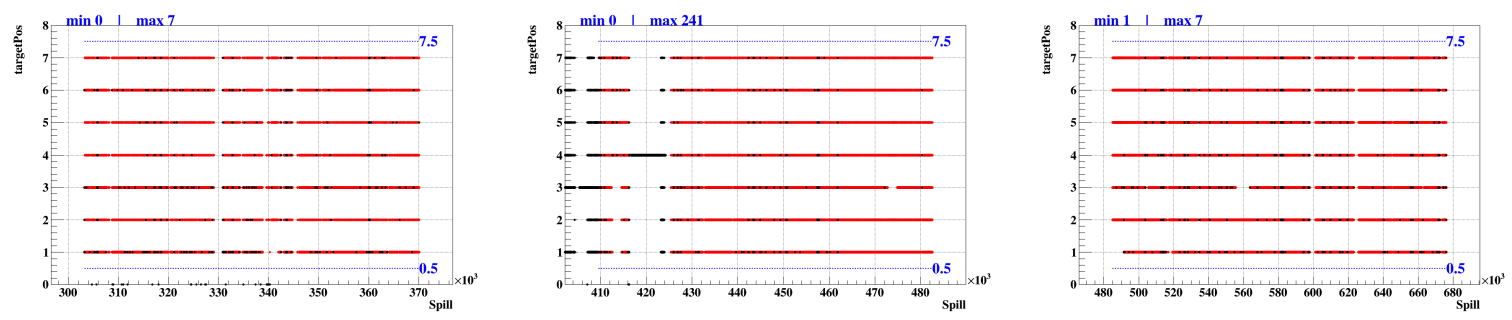

Figure 5.3: Target position vs spill ID for Roadset 57, 62 and 67 data from left to right. Target positions were not correctly recorded in the beginning of the Roadset 62 period due to a trouble of the software which was reading the target positions.

\section{Target position}

Figure 5.31 shows the target position as a function of spill ID. In the beginning of Roadset 62 data, target positions were not correctly recorded due to trouble of the target software which was reading the target positions. Since the target turn table itself was properly moving, and the positions were recorded in shifter's log by hand. So the appropriate positions will be assigned to those spills, and the data during the period will be analyzable in future. The remaining part of Roadset 62 data is analyzable and the software was running correctly.

\section{Beam intensity}

The beam intensity as a function of spill ID for each Roadset data is shown in Fig. 5.4. The top three figures show the intensity measured by G2SEM vs spill ID, the middle three shows the QIEsum vs spill ID, and the bottom three shows the ratio of G2SEM/QIEsum vs spill ID. The value measured by G2SEM is an absolute value of intensity while the QIE outputs its relative value as explained in the beam monitor section in chapter 4 . The beam intensity sometimes varied due to the accelerator condition during Roadset 57 period, and it became more stable during Roadset 62 period. The QIEsum behaves similarly to G2SEM, but the ratio of G2SEM/QIEsum gradually drops. This is because the mirror inside the Cherenkov counter becomes darker due to a beam halo, and the reflection efficiency then drops. 

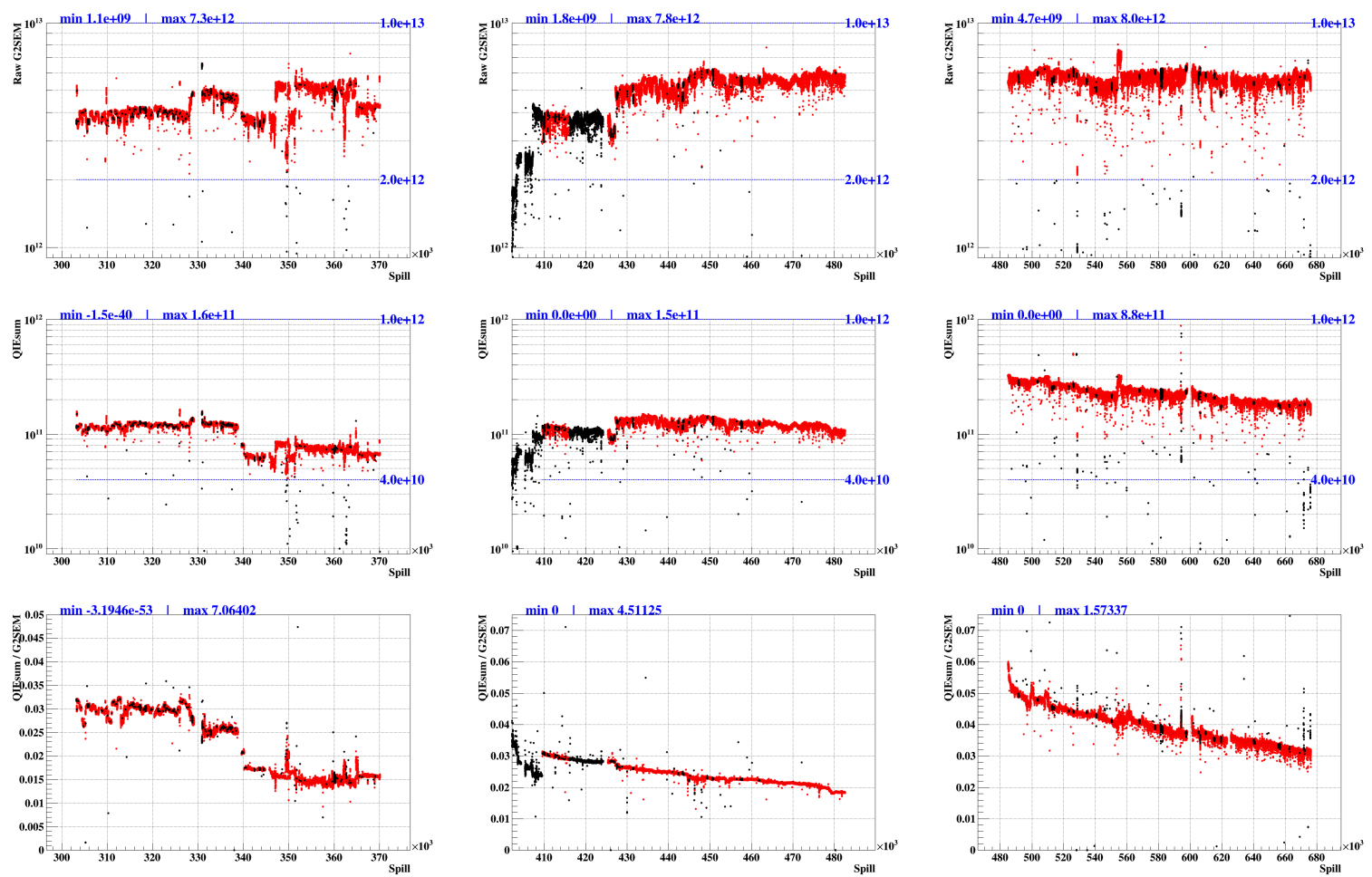

Figure 5.4: G2SEM vs spill ID (top), QIEsum vs spill ID (middle), QIEsum/G2SEM vs spill ID (bottom) for Roadset 57, 62 and 67 data from left to right. Good spills are shown in red and bad spills are shown in black. The QIEsum behaves similarly to G2SEM, but the ratio of G2SEM/QIEsum gradually drops. This is because the mirror inside the Cherenkov counter becomes darker due to beam halo and the reflection efficiency then drops. 

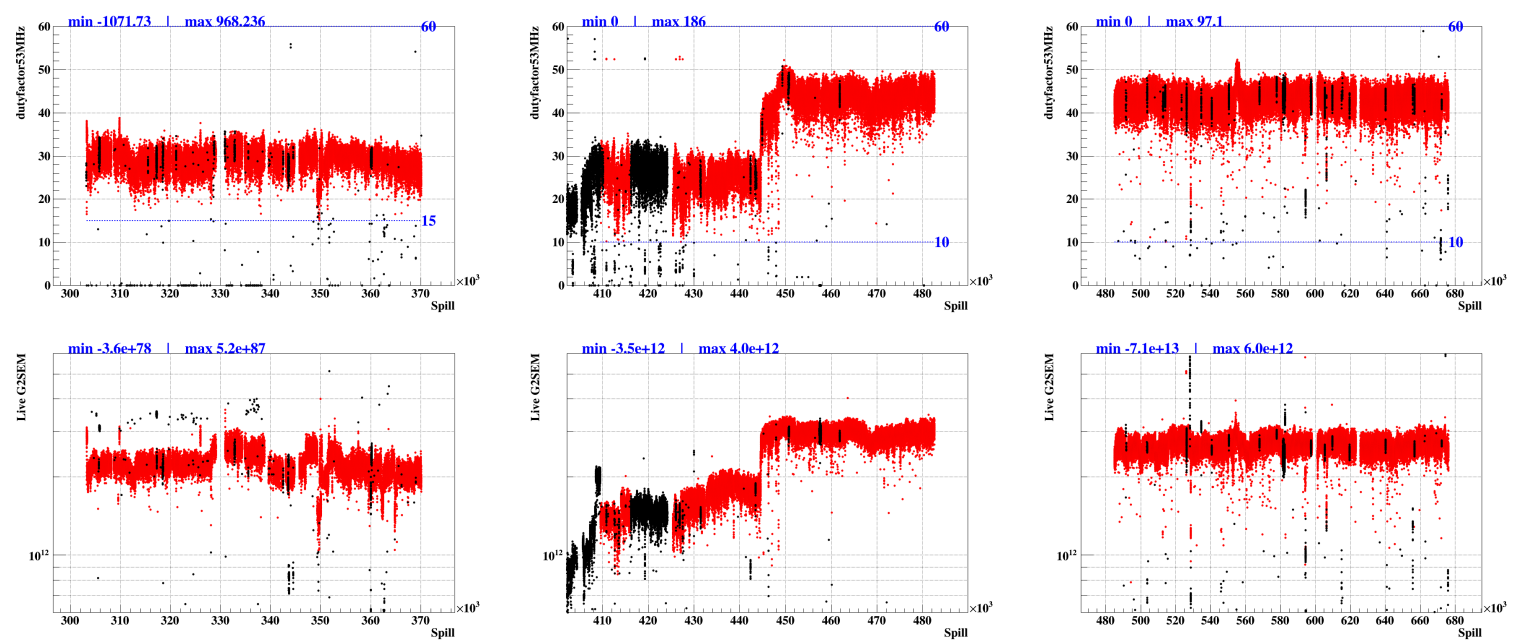

Figure 5.5: Duty factor vs spill ID (top), and live G2SEM vs spill ID (bottom) for Roadset 57, 62 and 67 data from left to right. Good spills are shown in red and bad spills are shown in black. Live G2SEM behaves similarly to the duty factor.

\section{Duty factor and RF status}

The top three figures in Fig. 5.5 show the duty factor as a function of spill ID. The duty factor was drastically improved during Roadset 62 period. Settings of the kicker magnet for the proton slow extraction system was changed, and it made the duty factor improved. The bottom three in Fig. 5.5 shows live G2SEM as a function of spill ID. Live G2SEM is sum of number of protons of "live" RF buckets (see Fig. 5.2). It usually behaves similarly to the duty factor. In Fig. 5.6, the top three figures show number of "busy" RF bucket vs spill ID, and the bottom three figures show number of "inhibited" $\mathrm{RF}$ bucket vs spill ID. If the number of protons was stable (in other words, duty factor is high), inhibited RF bucket usually decreases, and busy and live RF buckets increased, depending on the condition of data taking. 

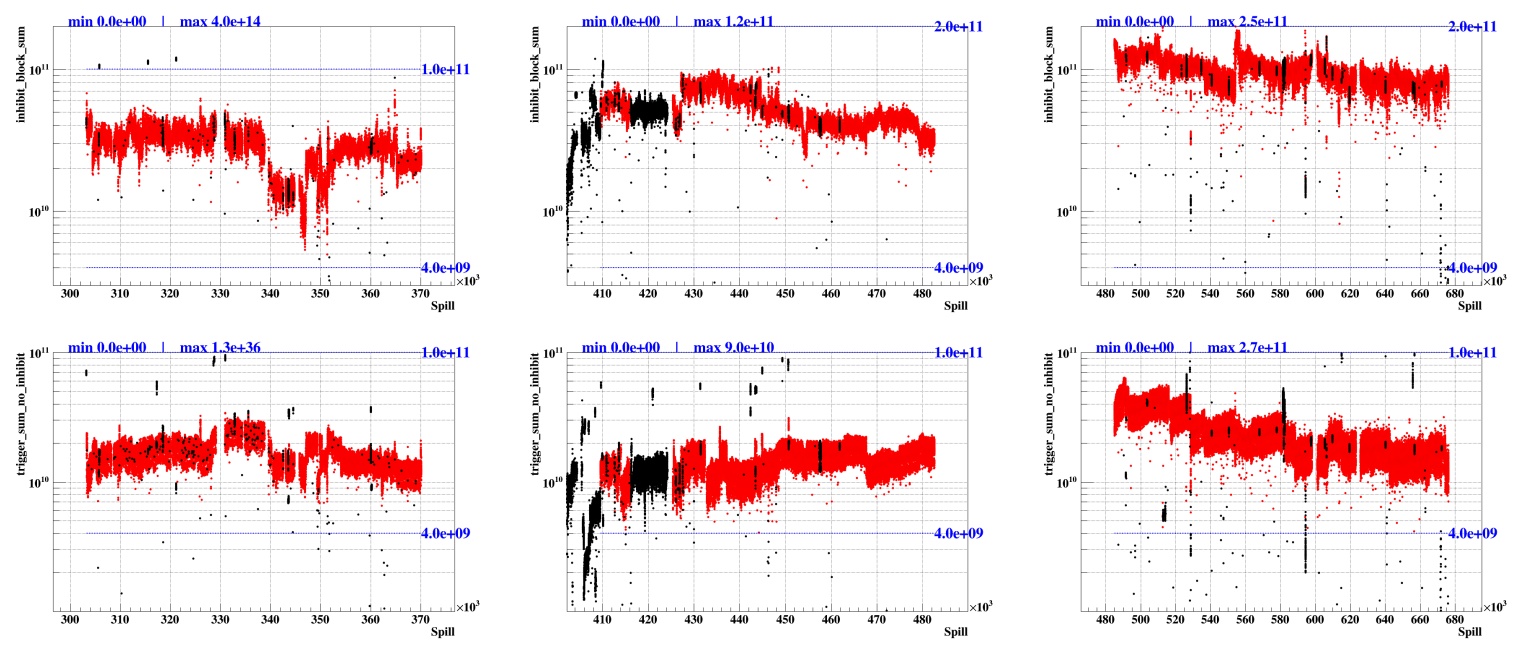

Figure 5.6: Busy vs spill ID (top), and inhibit vs spill ID (bottom) for Roadset 57, 62 and 67 data from left to right. Good spills are shown in red and bad spills are shown in black. When the duty factor is high, inhibited RF bucket usually decreases, and busy and live RF buckets increased, depending on the condition of data taking. 

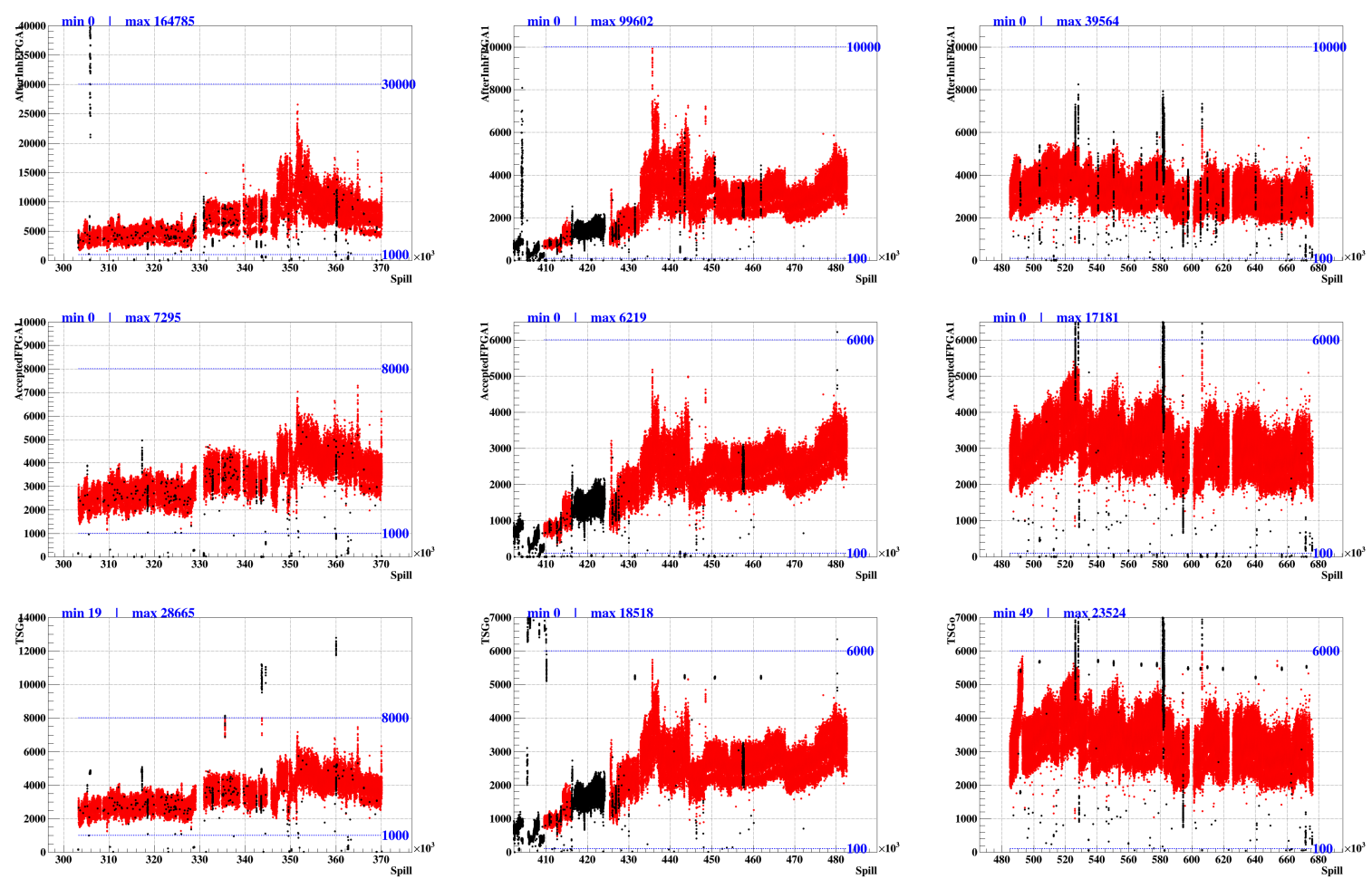

Figure 5.7: AfterInhFPGA1 vs spill ID for Roadset 57, 62 and 67 data. Good spills are shown in red and bad spills are shown in black. These are proportional to each other, and also proportional to the number of live protons.

\section{Trigger rate}

Figure 5.7 shows several trigger rate. The top three figures show AfterInhFPGA1 vs spill ID, the middle three show AcceptedInhFPGA1 vs spill ID, and the bottom three show TSGO vs spill ID. They are proportional to each other. Especially the TSGo rate is mostly equal to the AccepedFPGA1 rate because events by other triggers are scaled down. These three are obviously proportional to the number of live protons (Fig. 5.5). 


\subsubsection{Results of spill cuts}

Table 5.4 shows the result of the spill cut. Roughly $80 \%$ of spills are "good" spills in Roadset 57 and 67 data while it is $62 \%$ in Roadset 62 data. This is because of the target software trouble at the beginning of the Roadset 62 period as mentioned before.

\begin{tabular}{lllllll}
\hline \hline Category & \multicolumn{2}{c}{ Roadset 57 } & \multicolumn{2}{c}{ Roadset 62 } & \multicolumn{2}{c}{ Roadset 67 } \\
& $\#$ & $\%$ & $\#$ & $\%$ & $\#$ & $\%$ \\
\hline Bad spills & 14253 & 23 & 32671 & 38 & 38363 & 21 \\
Good spills & 47307 & 77 & 53257 & 62 & 144205 & 79 \\
\hline All spills & 61560 & & 85928 & & 182568 & \\
\hline
\end{tabular}

Table 5.4: Results of the spill cuts for the three Roadset data.

Number of spills, raw number of protons, live number of protons, and their ratio are listed for each Roadset in Table 5.5. Roughly half of the received protons are "live"

\begin{tabular}{llllll}
\hline \hline Roadset & Target & Good spills & Raw number of protons & Live number of protons & Live/Raw \\
\hline \multirow{3}{*}{ Roadset 57 } & LH2 & 20410 & $8.8510 \mathrm{e}+16$ & $4.6076 \mathrm{e}+16$ & 0.52 \\
& Empty & 2147 & $9.2349 \mathrm{e}+15$ & $5.0952 \mathrm{e}+15$ & 0.55 \\
& LD2 & 10831 & $4.6606 \mathrm{e}+16$ & $2.3098 \mathrm{e}+16$ & 0.50 \\
\hline \multirow{3}{*}{ Roadset 62 } & LH2 & 22573 & $1.1984 \mathrm{e}+17$ & $5.5781 \mathrm{e}+16$ & 0.47 \\
& Empty & 4474 & $2.3790 \mathrm{e}+16$ & $1.1599 \mathrm{e}+16$ & 0.49 \\
& LD2 & 11119 & $5.8278 \mathrm{e}+16$ & $2.5660 \mathrm{e}+16$ & 0.44 \\
\hline \multirow{2}{*}{ Roadset 67 } & LH2 & 60840 & $3.4573 \mathrm{e}+16$ & $1.6264 \mathrm{e}+17$ & 0.47 \\
& Empty & 13235 & $7.5294 \mathrm{e}+15$ & $3.6937 \mathrm{e}+16$ & 0.49 \\
& LD2 & 30398 & $1.7255 \mathrm{e}+16$ & $7,7572 \mathrm{e}+16$ & 0.45 \\
\hline
\end{tabular}

Table 5.5: Results of the spill cuts for Roadset 57, 62 and 67 data. Roughly half of the received protons are "live" protons.

protons. The empty target events has the highest Live/Raw rate, followed by LH2 and LD2 target events in this order. This is the order of the mass of the material in the target. If the target mass is high, the trigger rate becomes high, so "busy" time increased, and then the "live" time decreased. 


\subsection{Dimuon reconstruction}

All the events that passed the spill cuts are then analyzed to reconstruct muon tracks using a track reconstruction tool. The purpose of the track reconstruction is to determine the most accurate estimates of the track parameters [47]. The tool used for this purpose is "kTracker", where "k" comes from Kalman-Filter which is used in the vertex finding. The algorithm of the tracker is divided to three analysis stages: "Pre-tracking analysis", "Track reconstruction", and "Vertex finding". Those are described in the following subsections in detail. The procedure is shown in Fig. 5.8 .

\subsubsection{Pre-tracking analysis}

Before an event is passed to main reconstruction stage, following hit reduction methods are applied in order to remove noise hits and thus to reduce the computing time.

- Out-of-time hits removal: all the hits with TDC time being out of the predefined TDC time window are discarded.

- After-pulse removal: in each event, only the first pulse on each channel is accepted as signal, and the pulses coming after the first pulse are discarded.

Additionally, in the drift chambers, it is sometimes observed that contiguous wires are fired at the same time. This hit group is called "hit cluster". The source of the hit cluster is categorized into three types, and different treatments are applied to each in order to remove them:

- Hit pairs: in case of two neighboring wires both fired, one hit has the drift distance close to half the cell width, and the other hit also has a large drift distance. This hit pair is considered to be coming from one track that happens to cross the middle of two adjacent wires. Thus the hit with larger drift distance is discarded.

- Electronic noise: in case of more than two contiguous hits, if the average of difference of TDC time between neighboring cells is small (less than $10 \mathrm{~ns}$ ), all the hits are considered to be coming from electronic oscillations and thus discarded.

- $\delta$-ray induced clusters: in case of more than two contiguous hits, if the average difference of their TDC time is not small, they are considered to be induced by transversing $\delta$-ray, thus only the two hits on the both edges of the hit cluster are kept and the others hits are discarded. 


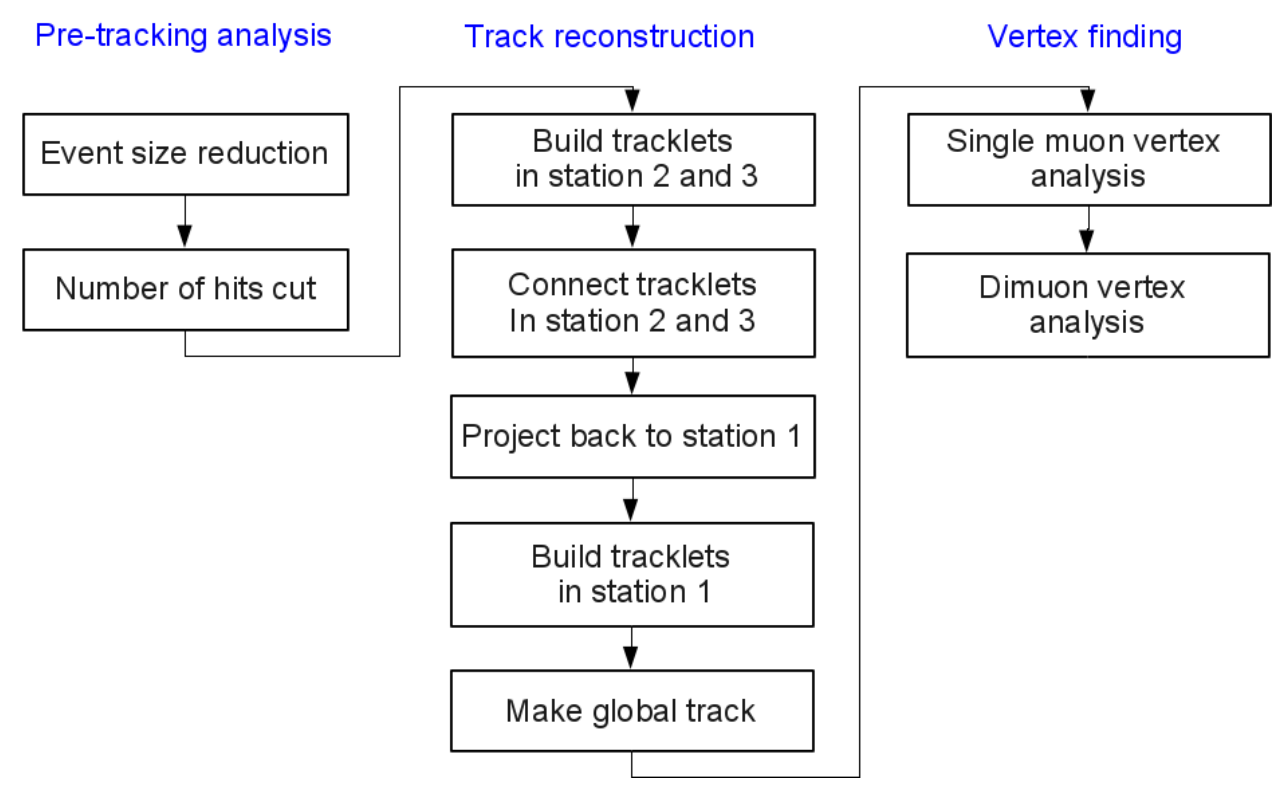

Figure 5.8: Procedure of the dimuon reconstruction using "kTracker". It consists of three parts as shown: Pre-tracking analysis part, track reconstruction part, and vertex finding part.

After these hit reduction cuts, number of hits per detector plane is checked in the next step. Cuts on number of hits (Table. 5.6l) are applied in order to remove the events which has too many hits and takes long time to be tracked.

\subsubsection{Track reconstruction}

\section{Tracklet finding in individual chamber}

A "tracklet" is defined as the local track inside each drift chamber (one drift chamber consists of 6 planes, namely the $\mathrm{X} / \mathrm{X}^{\prime}, \mathrm{U} / \mathrm{U}^{\prime}$ and $\mathrm{V} / \mathrm{V}^{\prime}$ planes). The track finding begins with looking for tracklets in D2, D3p and D3m. Since all the drift chambers have similar internal structure, one tracklet finding algorithm is implemented for all the drift chambers.

The tracklet search starts from $\mathrm{X} / \mathrm{X}^{\prime}$ view; for any hit on $\mathrm{X}$ view, if there exist a $\mathrm{X}^{\prime}$ hit with element ID next to the $\mathrm{X}$ hit, the pair of $\mathrm{X}$ and $\mathrm{X}$ ' hits is considered as a hit pair and used as the seed for tracklet searching. After all possible hit pairs are found, the remaining unpaired $\mathrm{X}$ or $\mathrm{X}$ ' hits are also added to the list of tracklet seeds. The second step is to associate the possible $\mathrm{U} / \mathrm{U}^{\prime}$ hits to $\mathrm{X} / \mathrm{X}^{\prime}$ seeds. For each $\mathrm{X} / \mathrm{X}$ ' tracklet seed, the search range for $\mathrm{U} / \mathrm{U}$ ' hits are reduced to those who could intersect with the given $\mathrm{X} / \mathrm{X}^{\prime}$ wire after track slope correction. The search window used in the tracker is defined 


\begin{tabular}{ll}
\hline \hline Detector plane & Upper limit of number of hits \\
\hline D1 & 300 \\
D2 & 200 \\
D3 plus & 180 \\
D3 minus & 160 \\
H1T \& H1B & 15 \\
H2T \& H2B & 10 \\
H3T \& H3B & 10 \\
H4T \& H4B & 10 \\
Proportional tube & 300 \\
\hline
\end{tabular}

Table 5.6: Cuts on number of hits cut per detector plane. Upper limit of the number of hits for each detector plane is listed.

as:

$$
u_{\text {win }}=\frac{1}{2} L_{x} \sin \theta+t_{x}^{\max }\left|z_{u}-z_{x}\right| \cos \theta+t_{y}^{\max }\left|z_{u}-z_{x}\right| \sin \theta+2 \Delta u+\delta,
$$

where $L_{x}$ is the length of $\mathrm{X}$ wire, $t_{x}^{\max }$ and $t_{x}^{\max }$ the maximum tracklet slopes in $\mathrm{X}-\mathrm{Z}$ and $\mathrm{Y}-\mathrm{Z}$ view, $z_{u}$ and $z_{x}$ the $z$ position of the $\mathrm{U}$ and $\mathrm{X}$ planes, $\theta$ the tilting angle of $\mathrm{U}$ view, $\Delta u$ the wire spacing of $\mathrm{U}$ view, and $\delta$ the extra contingency window which is set to be 5 $\mathrm{cm}$ for D1 and D2, and $10 \mathrm{~cm}$ for D3p and D3m. For each X tracklet seed, there might be multiple $\mathrm{U}$ hits or hit pairs in the window, so all possible combinations are formed and passed to the next step. The third step in tracklet search is to find the appropriate $\mathrm{V} / \mathrm{V}$ ' hits for each tracklet with $\mathrm{X}$ and $\mathrm{U}$ hits found. The procedure is similar to associating $\mathrm{U}$ hits, except that since $\mathrm{X}$ and $\mathrm{U}$ coordinates can already define a 3D point, the search window is very well confined to a small region.

After a tracklet with hits coming from X, U and V views are found, four parameters are used to describe the tracklet: $t_{x}$ and $t_{y}$ define the slope of the tracklet in X-Z and Y-Z views, and $x_{0}$ and $y_{0}$ are the projection of the track at $z=0$. A $\chi^{2}$ fit is applied assuming the resolution to be wire spacing divided by $\sqrt{12}$ to roughly determine the track parameters. Following cuts are made to reject the tracklets with bad qualities:

- $\left|t_{x}\right|<t_{x}^{\max },\left|t_{y}\right|<t_{y}^{\max },\left|x_{0}\right|<x_{0}^{\max },\left|y_{0}\right|<y_{0}^{\max }$

- $\chi^{2}<15$

- $N_{\text {hits }}>4$, and $N_{\text {hits }}$ on each view $(\mathrm{X} / \mathrm{U} / \mathrm{V})$ should be no less than 1

The tracklet is projected to the nearest top/bottom hodoscope and require the corresponding paddle to be on. At this stage, tracklets are allowed to share common hits of a paddle. 


\section{Forming back partial tracks in Station 2 and 3}

After the hits of tracklets in D2, D3p and D3m are prepared, all the possible combinations of Station 2 and Station 3 tracklets are connected to see if they could be a long straight track, which is denoted as a "back partial track". Before the connection, the X hits in both Station 2 and 3 tracklets are used to make a fast calculation of the X-Z slope and intersection. The tracklets are then rejected if the slope is larger than the maximum slope. They are also projected back to proportional tube, and require at least one hit on the extrapolated position. $\chi^{2}$ fit as used in tracklet finding is then applied on each possible combination.

With the connection of D2 and D3 tracklets, it is tried to resolve the left-right ambiguity. For each hit pair, the local slope and intersection are calculated for all possible drift sign assignments (left-left, left-right, right-left, right-right). These values are first compared with the maximum possible value to reject the impossible values. The remaining drift sign assignments are then compared using $\chi^{2}$, and the best guess is selected. After looping over all the hit pairs, another $\chi^{2}$ fit is applied with updated drift sign assignment. The whole procedure is repeated after the fit as the track parameter has been improved.

\section{Building global tracks}

With all the back partial tracks reconstructed, each track is projected back to Station 1 drift chambers through KMAG (downstream magnet) to pair with the appropriate Station 1 tracklets. The search window is constrained by the ratio of sagitta on Station 2 and Station 1, as defined in Fig. 5.9. The range of sagitta ratio is $s_{1} / s_{2}=1.77 \pm 0.2$, which was defined by Monte Carlo simulation. All possible Station 1 tracklets are connected to the back partial track to form a global track candidate.

The track after Station 1-, 2-, and 3-tracklets are connected is called "global track". Each global track candidate is fed to a $\chi^{2}$ fitter with left-right ambiguity of Station 1 tracklet. If there are hits that have a large residual (larger than three times of the chamber position resolution), the hit with the largest residual is removed and the track is re-fitted to update the residual of each hit. This procedure is repeated until the residuals of all remaining hits become smaller than three times of the chamber position resolution. 


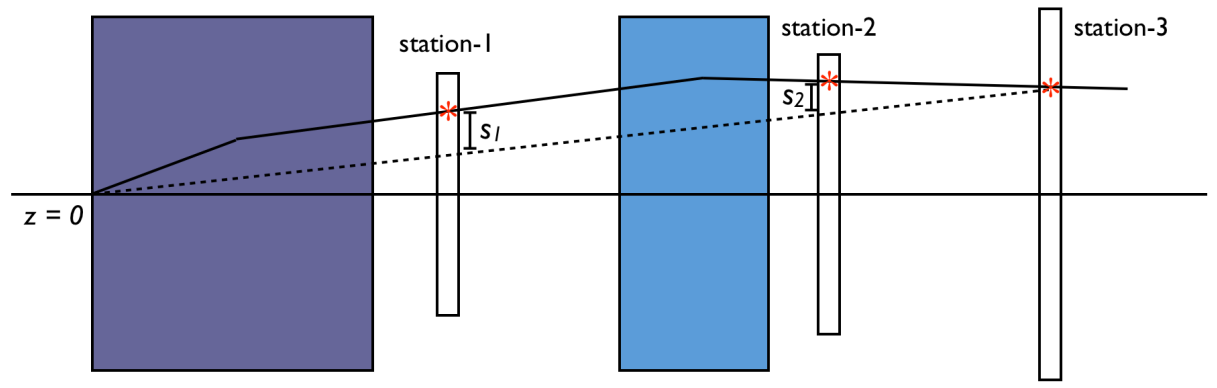

Figure 5.9: Definition of sagitta ratio. For $\mathrm{X} / \mathrm{U} / \mathrm{V}$ views, $s_{1} / s_{2}=1.77 \pm 0.2$ for the SeaQuest spectrometer. 


\subsubsection{Vertex fitting}

After all the tracks are found and fitted in the detector, all the good global tracks are propagated through FMAG (upstream magnet) to find its primary reaction vertex.

\section{Simple tracing back through FMAG}

A simple tracing back algorithm assuming perfectly uniform dipole magnetic field is developed to find the muon vertex based on single tracks. The whole length of FMAG is divided to 100 slices, in each slice, a simple $p_{T}$ kick is applied at the center of the slice as illustrated in Fig. 5.10. The energy loss correction is also applied at both first half and the second half of the tracing in each slice. Figure 5.1 shows how the penetrating muon loses its momentum in FMAG. The muon having $30 \mathrm{GeV} / \mathrm{c}$ which is a typical value is drawn as an example. The magnitude of the energy loss inside FMAG $(\sim 500 \mathrm{~cm})$ is calculated by

$$
E_{\mathrm{loss}}=7.1827+0.036145 p-0.00071813 p^{2}
$$

where $p$ is the momentum of the penetrating muon. The coefficients are obtained by GMC. The vertex point of the track is then a point where the distance to $z$-axis is minimum.

\section{Di-muon vertex finding}

The single muon tracking back method is applied to both $\mu^{+}$and $\mu^{-}$global track, and two vertex positions are given. First guess of dimuon vertex position is calculated from the given two of single muon vertex. It is an average of their positions. The guess is then fitted and updated by Kalman-Filter method. The iteration will continue until the $\chi^{2}$ stays and the result passes quality check.

If an event has more than one $\mu^{+}$or $\mu^{-}$track, all the combination of $\mu^{+}$and $\mu^{-}$are tried with the Kalman-Filter method. All the dimuons that passed the quality check are stored to database. 


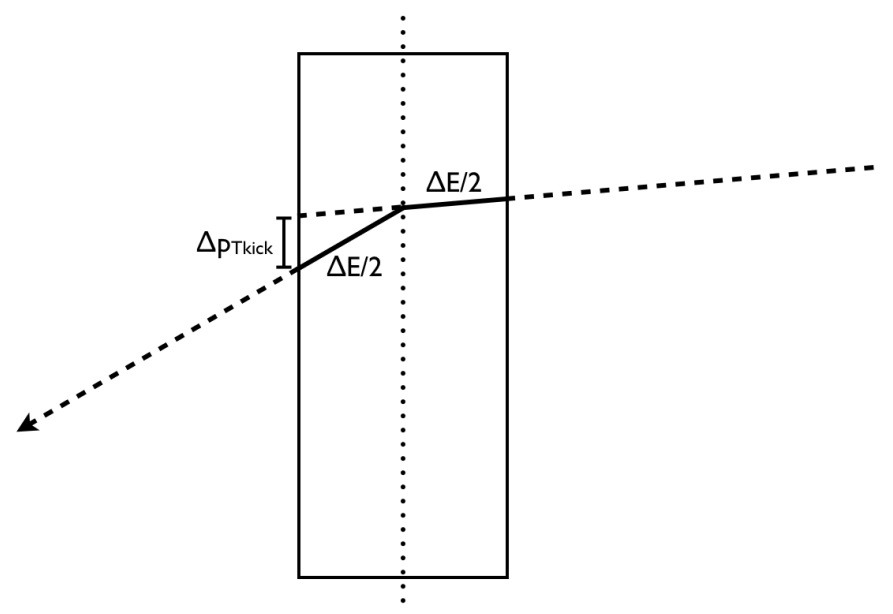

Figure 5.10: One step of simple tracing back through FMAG. It is assumed that the track is kicked at the center of the sliced space and got $\Delta_{P T k i c k}$ there.

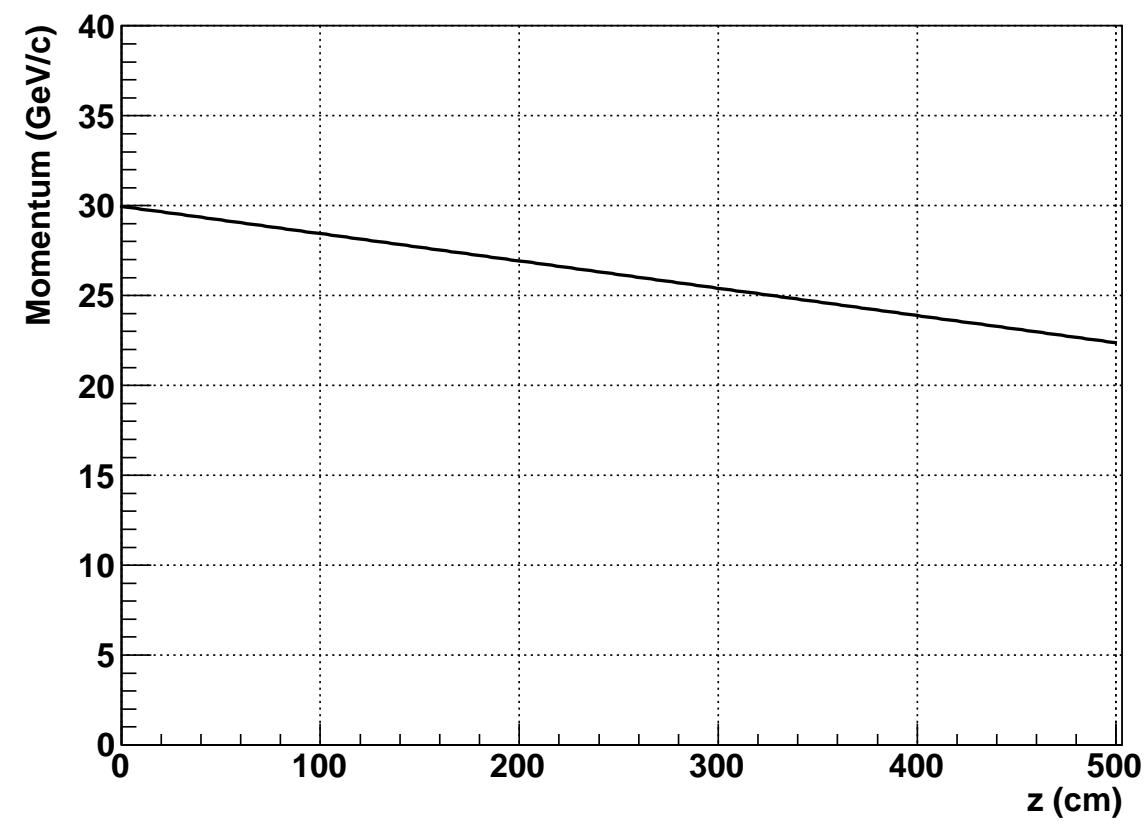

Figure 5.11: Muon momentum as a function of $z$ in FMAG. A muon having $30 \mathrm{GeV} / \mathrm{c}$ before penetrating into FMAG is simulated as as example. 


\subsection{Track selection}

Track and dimuon selections are applied to all the obtained dimuons. Table 5.7 shows the track selection. Those cuts are applied to both $\mu^{+}$and $\mu^{-}$track of dimuon, respectively. Since one drift chamber has six planes, one track can have 18 associated hits on it at maximum. The last cut for the $z$-momentum is applied only when the track has less than associated 18 hits.

\begin{tabular}{lll}
\hline \hline Quantity & Min. & Max. \\
\hline Number of hits & 15 & 18 \\
Reduced chi-square & 0.0 & 5.0 \\
$z$-vertex position $(\mathrm{cm})$ & -400.0 & 200.0 \\
$p_{z}($ when number of associated hits $<18)$ & 18.0 & - \\
\hline
\end{tabular}

Table 5.7: Cuts for track. The last cut for the $z$-momentum is applied only when the track has less than associated 18 hits.

\subsection{Dimuon selection}

\subsubsection{Kinematics cuts}

Kinematics cuts are applied to the dimuon events whose both $\mu^{+}$and $\mu^{-}$tracks passed the track cuts. The kinematics cuts are listed in Table 5.8. "Track separation" is the distance between the $z$-vertex of $\mu^{+}$and that of $\mu^{-} \cdot p_{x}^{\mu^{+}}$and $p_{x}^{\mu^{-}}$are the $x$-momentum of $\mu^{+}$and $\mu^{-}$track, respectively. Dimuon invariant mass $>4.2 \mathrm{GeV} / \mathrm{c}^{2}$ cut is applied in order to select only dimuons via Drell-Yan process. 


\begin{tabular}{lll}
\hline \hline Quantity & Min. & Max. \\
\hline$x$-vertex position $(\mathrm{cm})$ & -2.0 & 2.0 \\
$y$-vertex position $(\mathrm{cm})$ & -2.0 & 2.0 \\
$z$-vertex position $(\mathrm{cm})$ & -300.0 & 200.0 \\
Reduced $\chi$ square of track reconstruction & 0.0 & 5.0 \\
$x$-momentum at vertex $(\mathrm{GeV} / \mathrm{c})$ & -3.0 & 3.0 \\
$y$-momentum at vertex $(\mathrm{GeV} / \mathrm{c})$ & -3.0 & 3.0 \\
$z$-momentum at vertex $(\mathrm{GeV} / \mathrm{c})$ & 30.0 & 120.0 \\
Bjorken $x_{1}$ & 0.0 & 1.0 \\
Bjorken $x_{2}$ & 0.0 & 1.0 \\
$x_{F}$ & -1.0 & 1.0 \\
Track separation & -250.0 & 250.0 \\
$\chi$ square of vertex reconstruction & 0.0 & 15.0 \\
$p_{x}^{\mu^{+}}(\mathrm{GeV} / \mathrm{c})$ & 0.0 & - \\
$p_{x}^{\mu^{-}}(\mathrm{GeV} / \mathrm{c})$ & - & 0.0 \\
\hline Dimuon invariant mass $\left(\mathrm{GeV} / \mathrm{c}^{2}\right)$ & 4.2 & - \\
\hline
\end{tabular}

Table 5.8: Kinematics cuts for dimuon. 


\subsubsection{Target-dump separation}

It is important to separate the dimuons produced at the SeaQuest target and at the beam dump (FMAG). Two variables are defined for the target-dump separation: $\chi_{\text {dump }}^{2}$ and $\chi_{\operatorname{targ}}^{2} \cdot \chi_{\text {dump(targ) }}^{2}$ is the $\chi^{2}$ of track reconstruction when forcing the vertex points to be at dump (target) region. $z$-position is forced to be $z=42(\mathrm{~cm})$ for the dump region and $z=-129.5(\mathrm{~cm})$ for the target region. Both values are calculated for each event. If

$$
\chi_{\text {dump }}^{2}-\chi_{\text {targ }}^{2}>10
$$

the event is regarded as the event produced at the target region, and if

$$
\chi_{\text {targ }}^{2}-\chi_{\text {dump }}^{2}>10
$$

the event is regarded as the event produced at the dump region [41]. Figure 5.12 shows $\chi_{\text {dump }}^{2}-\chi_{\text {targ }}^{2}$ as a function of $p_{z}$ of dimuon. This is the result using GMC. Red points indicate the dimuon events produced at the target region, and black points indicate the dimuon events produced at the dump region. The blue lines indicate the target-dump separation cuts. The target dimuon events and dump dimuon events are clearly separated by the cuts. Figure 5.53 shows the $z$-vertex distribution. This is also the result using GMC. Black line indicates all the dimuon events. Red(blue) line indicates the dimuons produced at the target(dump) region and passed the cuts for the target(dump) dimuon events, Eq. 5.3(Eq. 5.4). As shown in the figure, the target dimuon events and dump dimuon events are clearly separated by the cuts. Also, it can be seen that most of the target dimuon events passed the target cut. For the analysis in this thesis, only the dimuon events that passed the target event cut, Eq. 5.3, are used. 


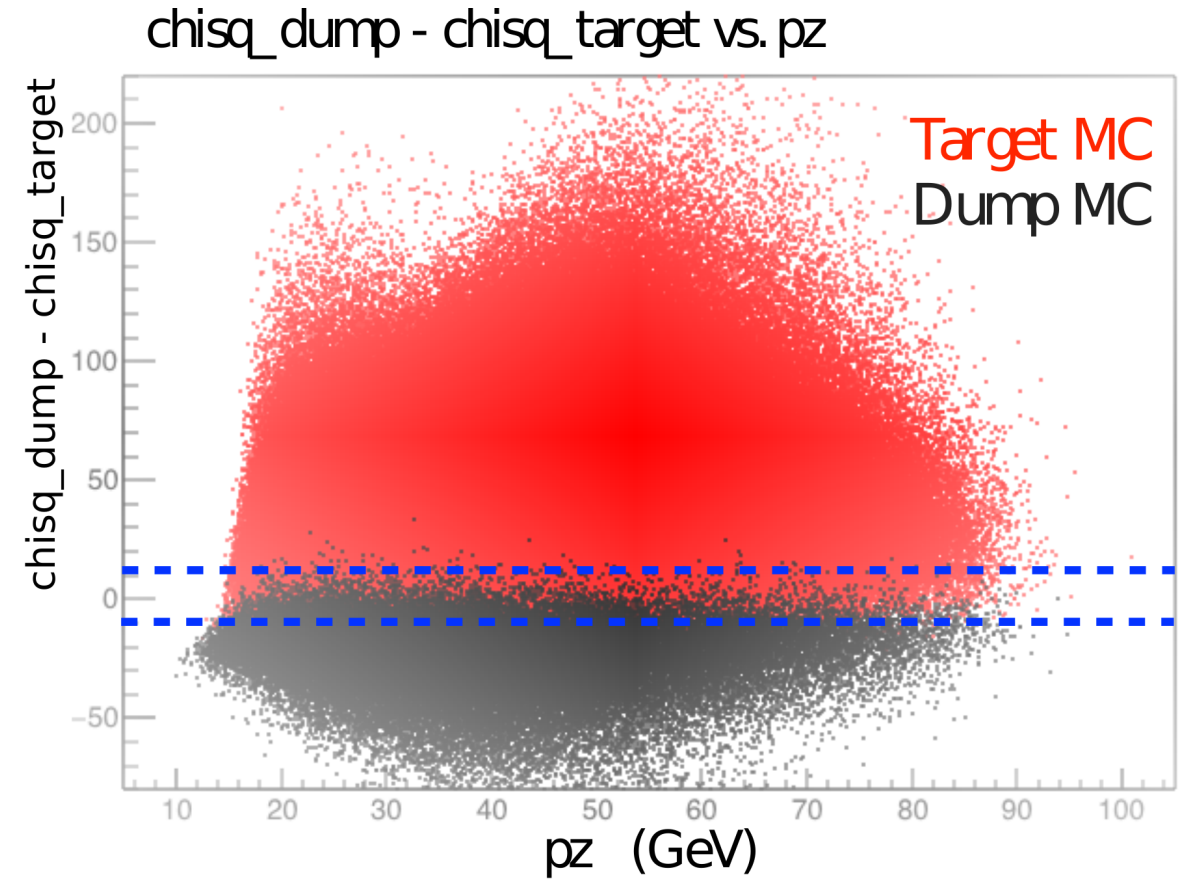

Figure 5.12: $\quad \chi_{\text {dump }}^{2}-\chi_{\text {targ }}^{2}$ as a function of $p_{z}$ of dimuon [41]. This is the result using GMC. Red points indicate the dimuon events produced at the target region, and black points indicate the dimuon events that produced at the dump region. The blue lines indicate the target-dump separation cuts. 


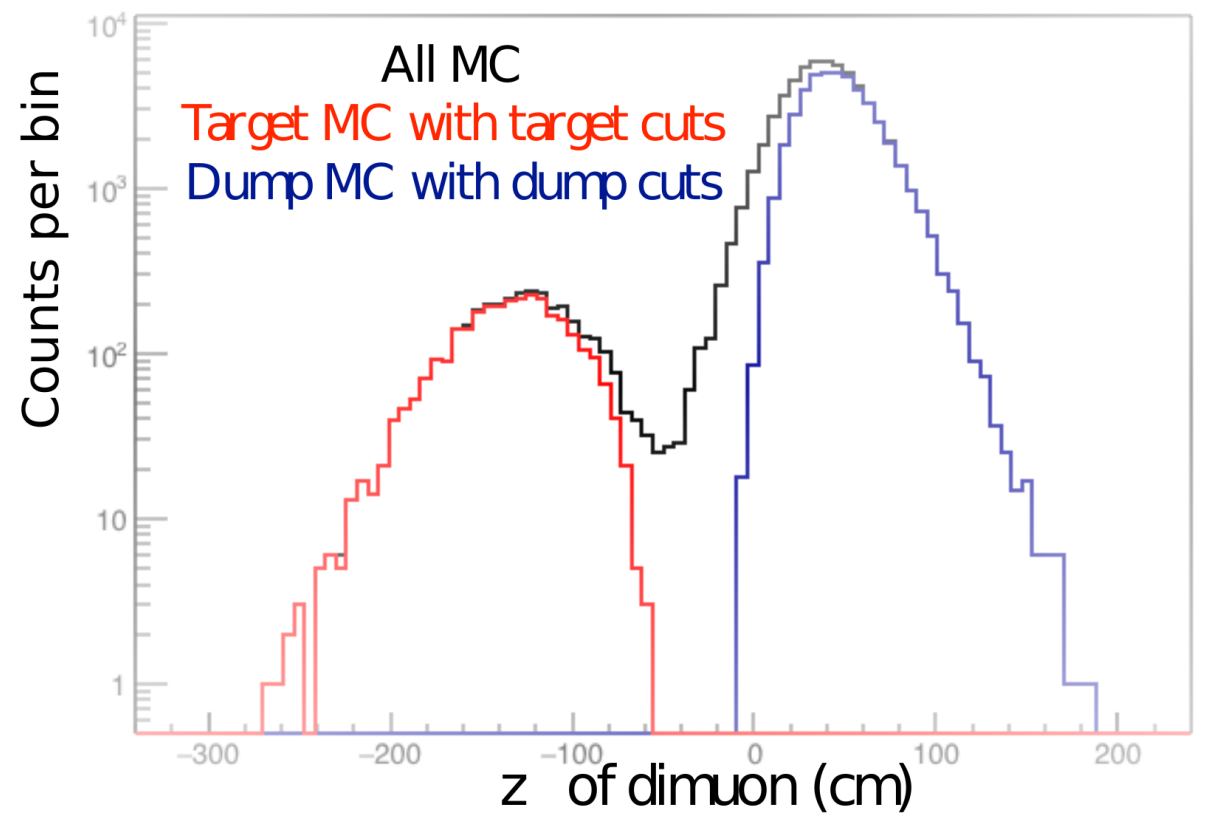

Figure 5.13: $z$-vertex distribution using GMC [41]. Black line indicates all the dimuon events. Red(blue) line indicates the dimuons produced at the target(dump) region and passed the cuts for the target(dump) dimuon events, Eq. 5.3(Eq. 5.4). 


\subsection{Dimuon yield}

The number of dimuons in each $x_{2}$ bin after the track and dimuon selection for Roadset

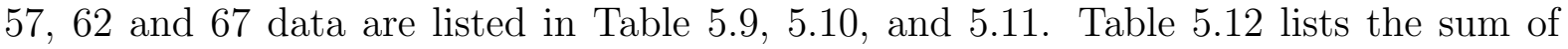
the number of reconstructed dimuons of all the Roadset data (Roadset 57, 62 and 67). Summed numbers over $x$ are also shown at the last row in the table.

\begin{tabular}{lll|lll}
\hline \hline Bin & x Min & x Max & LH2 & LD2 & Empty \\
\hline 1 & 0.100 & 0.130 & 242 & 282 & 6 \\
2 & 0.130 & 0.160 & 666 & 758 & 17 \\
3 & 0.160 & 0.195 & 782 & 873 & 15 \\
4 & 0.195 & 0.240 & 593 & 671 & 9 \\
5 & 0.240 & 0.290 & 322 & 354 & 17 \\
6 & 0.290 & 0.350 & 173 & 185 & 5 \\
7 & 0.350 & 0.450 & 61 & 88 & 5 \\
8 & 0.450 & 0.580 & 8 & 15 & 0 \\
\hline
\end{tabular}

Table 5.9: Number of reconstructed dimuons after analysis cuts in each $x_{2}$ bin in Roadset 57 data.

\begin{tabular}{lll|lll}
\hline \hline Bin & x Min & x Max & LH2 & LD2 & Empty \\
\hline 1 & 0.100 & 0.130 & 386 & 386 & 15 \\
2 & 0.130 & 0.160 & 1006 & 1018 & 49 \\
3 & 0.160 & 0.195 & 1254 & 1277 & 57 \\
4 & 0.195 & 0.240 & 998 & 960 & 47 \\
5 & 0.240 & 0.290 & 577 & 472 & 15 \\
6 & 0.290 & 0.350 & 269 & 270 & 11 \\
7 & 0.350 & 0.450 & 106 & 121 & 9 \\
8 & 0.450 & 0.580 & 18 & 14 & 0 \\
\hline
\end{tabular}

Table 5.10: Number of reconstructed dimuons after analysis cuts in each $x_{2}$ bin in Roadset 62 data. 


\begin{tabular}{lll|lll}
\hline \hline Bin & x Min & x Max & LH2 & LD2 & Empty \\
\hline 1 & 0.100 & 0.130 & 820 & 1020 & 42 \\
2 & 0.130 & 0.160 & 2285 & 2688 & 98 \\
3 & 0.160 & 0.195 & 2720 & 3322 & 128 \\
4 & 0.195 & 0.240 & 2223 & 2526 & 91 \\
5 & 0.240 & 0.290 & 1155 & 1438 & 58 \\
6 & 0.290 & 0.350 & 596 & 684 & 27 \\
7 & 0.350 & 0.450 & 248 & 291 & 10 \\
8 & 0.450 & 0.580 & 54 & 45 & 1 \\
\hline
\end{tabular}

Table 5.11: Number of reconstructed dimuons after analysis cuts in each $x_{2}$ bin in Roadset 67 data.

\begin{tabular}{lll|lll}
\hline \hline Bin & x Min & x Max & LH2 & LD2 & Empty \\
\hline 1 & 0.100 & 0.130 & 1448 & 1688 & 63 \\
2 & 0.130 & 0.160 & 3957 & 4464 & 164 \\
3 & 0.160 & 0.195 & 4756 & 5472 & 200 \\
4 & 0.195 & 0.240 & 3814 & 4157 & 147 \\
5 & 0.240 & 0.290 & 2054 & 2264 & 90 \\
6 & 0.290 & 0.350 & 1038 & 1139 & 43 \\
7 & 0.350 & 0.450 & 415 & 500 & 24 \\
8 & 0.450 & 0.580 & 80 & 74 & 1 \\
\hline Summed over $x$ & 0.100 & 0.58 & 17562 & 19758 & 732 \\
\hline
\end{tabular}

Table 5.12: Sum of the number of reconstructed dimuons of all the Roadset data (Roadset 57, 62 and 67). Summed numbers over $x$ are also shown at the last row. 


\subsection{Basic information of dimuon events}

In this section, basic information of obtained dimuon events are shown, such as kinematics distribution, track position distribution, and drift chamber occupancy. These are all the results from data.

\subsubsection{Mass distribution}

Distributions of invariant mass of dimuons are shown in Fig. 5.14 for Roadset 57, 62 and 67 data. Mass cut $\left(M>4.2 \mathrm{GeV} / \mathrm{c}^{2}\right)$ was not applied. The blue points show the yield of LD2 events, and the red points show that of LH2 events, and black points show that of empty target events. The yields are normalized by the number of live beam protons. The LD2 yield is roughly double of that of LH2 as the density of LD2 is about twice larger than LH2 as expected. This fact can be seen in other following kinematics distributions as well. Low mass events are drastically suppressed as intended because of the acceptance of the SeaQuest trigger logic described in the trigger section. The dimuon events are then categorized into four signal types: dimuon from $J / \psi$ decay, dimuon from $\psi^{\prime}$ decay, dimuon from Drell-Yan process, and muon pair of combinatorial background. Those are normalized by fitting to the data points. Their shapes are simulated by Monte Carlo events or real data events.

\section{Combinatorial background}

The combinatorial background is a muon pair from two single muons found in the same trigger event (in the triggered RF bucket) and accidentally combined in the track reconstruction process. The background was reproduced using data in the following way. The technique of event mixing is used. First, single muon tracks are collected for this purpose. Those single muon tracks are the tracks reconstructed in an event fired by dimuon trigger, FPGA-1, but that only has one track found. Next, opposite charged tracks from different events are randomly selected from the group of the single muon tracks. No tracks are used twice in this procedure. The muon pairs are combined as a "dimuon", and they are sent to the vertex analysis. The muon pair that passed the vertex analysis are regarded as the combinatorial background.

\section{Mass fitting}

Mass shape of dimuon events of Drell-Yan, $J / \psi, \psi^{\prime}$ and combinatorial background in Fig. 5.14 are fitted in order to break down the shape as shown in Fig. 5.15. The black 

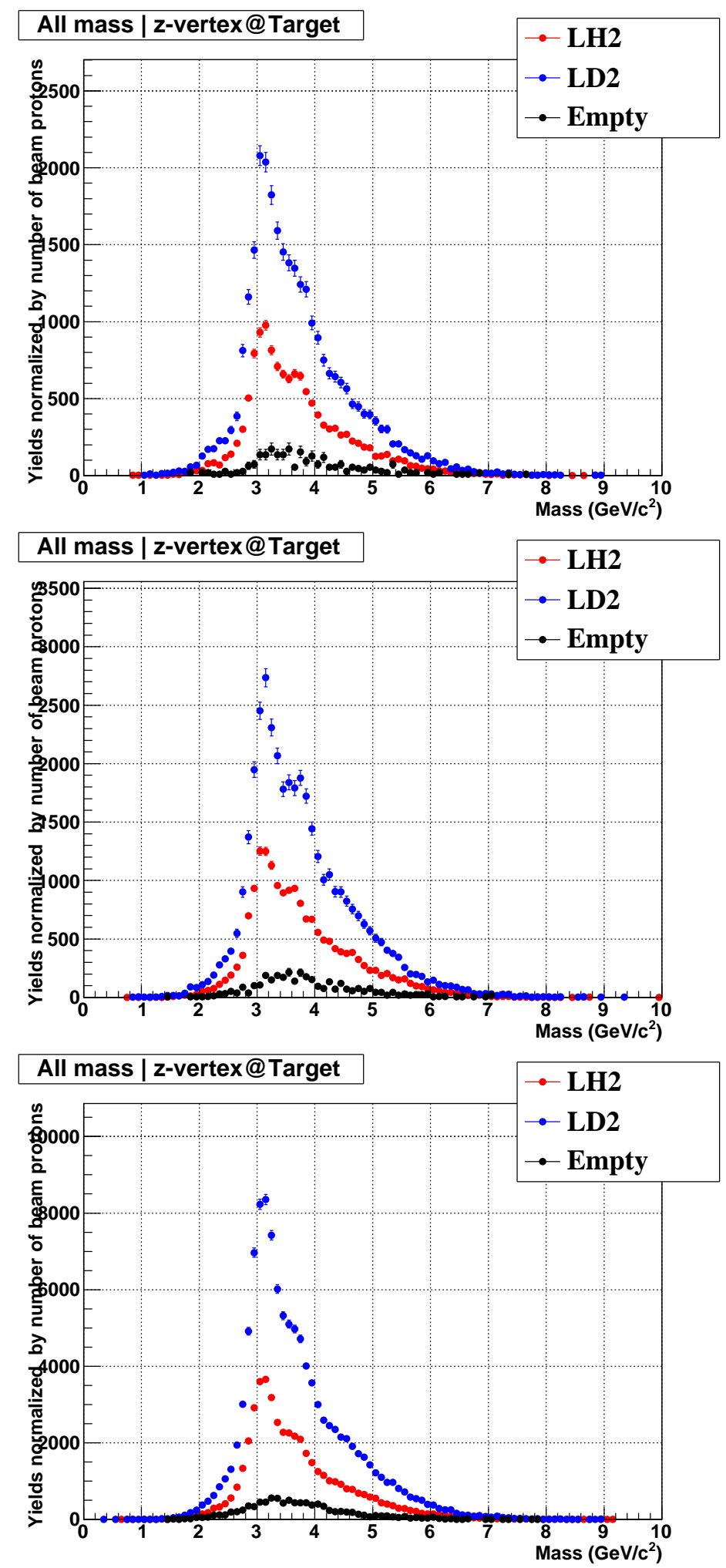

Figure 5.14: Mass distribution for each Roadset data. The top figure is from the Roadset 57 data, the middle figure from the Roadset 62 data, and the bottom figure from the Roadset 67 data. The blue points show yield of LD2 events, the red points show that of LH2 events, and black points show that of empty target events. The yields are normalized by number of live beam protons. The LD2 yield is roughly double of that of LH2 as the density of LD2 is about twice larger than LH2 as expected. Low mass events are drastically suppressed because of the acceptance of the SeaQuest trigger logic. 


\section{TargetPos: 1}

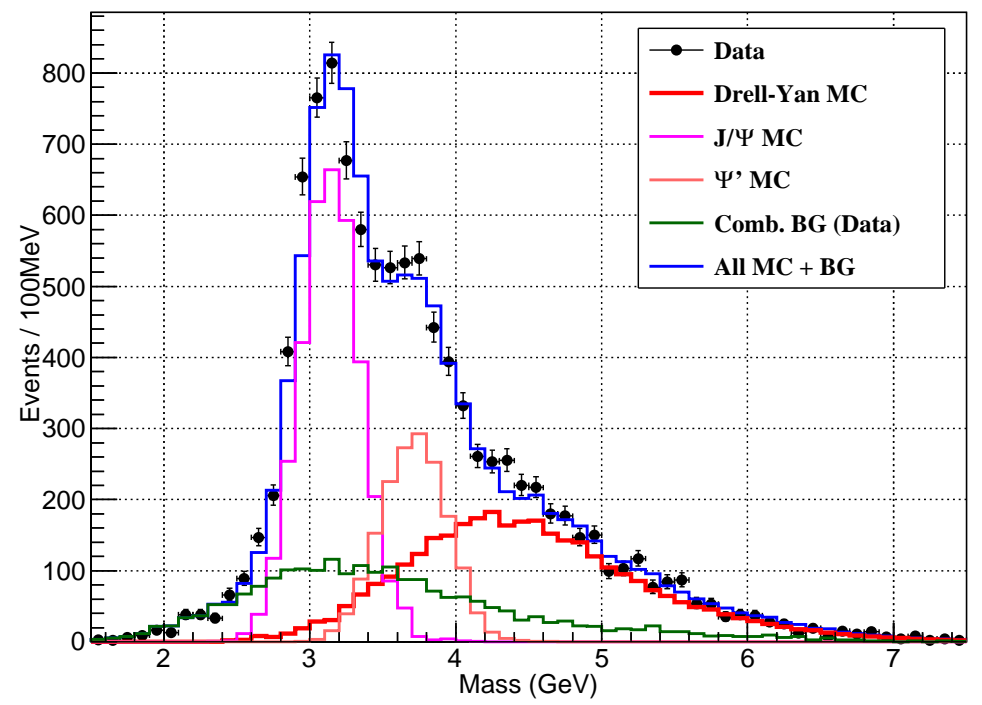

Figure 5.15: The black points and error bars show the data. The red, magenta and orange lines show Monte Carlo data of Drell-Yan, $J / \Psi, \Psi^{\prime}$ events, respectively. The green line shows combinatorial background. Its shape was determined by real data.

points and error bars show the data. The red, magenta and orange lines show Monte Carlo data of Drell-Yan, $J / \psi, \psi^{\prime}$ events, respectively. The green line shows combinatorial background. The blue line is the sum of the four components. The black points are the data. The fitting was successfully done as can be seen in the figure. The reduced $\chi^{2}$ of the fitting is around 2.0. By looking at the fit result, the Drell-Yan events are dominant at Mass $>4.2 \mathrm{GeV}$ except for the combinatorial background. This is the reason why the mass cut of $4.2 \mathrm{GeV}$ is applied in the dimuon selection (Table 5.8) in order to select Drell-Yan events. 


\subsubsection{Bjorken $x$ distribution}

Figure 5.16 shows the distributions of Bjorken $x_{1}$, Bjorken $x_{2}$ and two dimensional plots of Bjorken $x_{2}$ vs Bjorken $x_{1}$. Looking into the $x_{2}$ distribution, high $x_{2}$ events $\left(x_{2}>0.3\right)$ are obtained as designed. A straight line of $x_{2}=x_{1}$ is drawn in the $x_{2}$ vs $x_{1}$ figure. The most events exist in the area of $x_{2}<x_{1}$, namely $x_{F}>0$, since the SeaQuest experiment is a fixed-target experiment and muons in the forward direction are measured. Also, a hyperbolic curve of $M=4.2 \mathrm{GeV} / \mathrm{c}^{2}$ is drawn on the $2 \mathrm{D}$ plot. The events obviously exist above the curve due to the applied mass cut in the dimuon selection. There is no visible difference among the three Roadset data.

\subsection{3 z-vertex distribution}

Figure 5.17 shows the $z$-vertex distributions for Roadset 57, 62 and 67. The SeaQuest target is placed from $z=-150$ to $z=-100(\mathrm{~cm})$. The peaks of the distributions of LH2 and LD2 target events are reasonably placed at the center of the target position. It can be seen that z-vertex distribution is dominant upstream in the case of the empty target. 

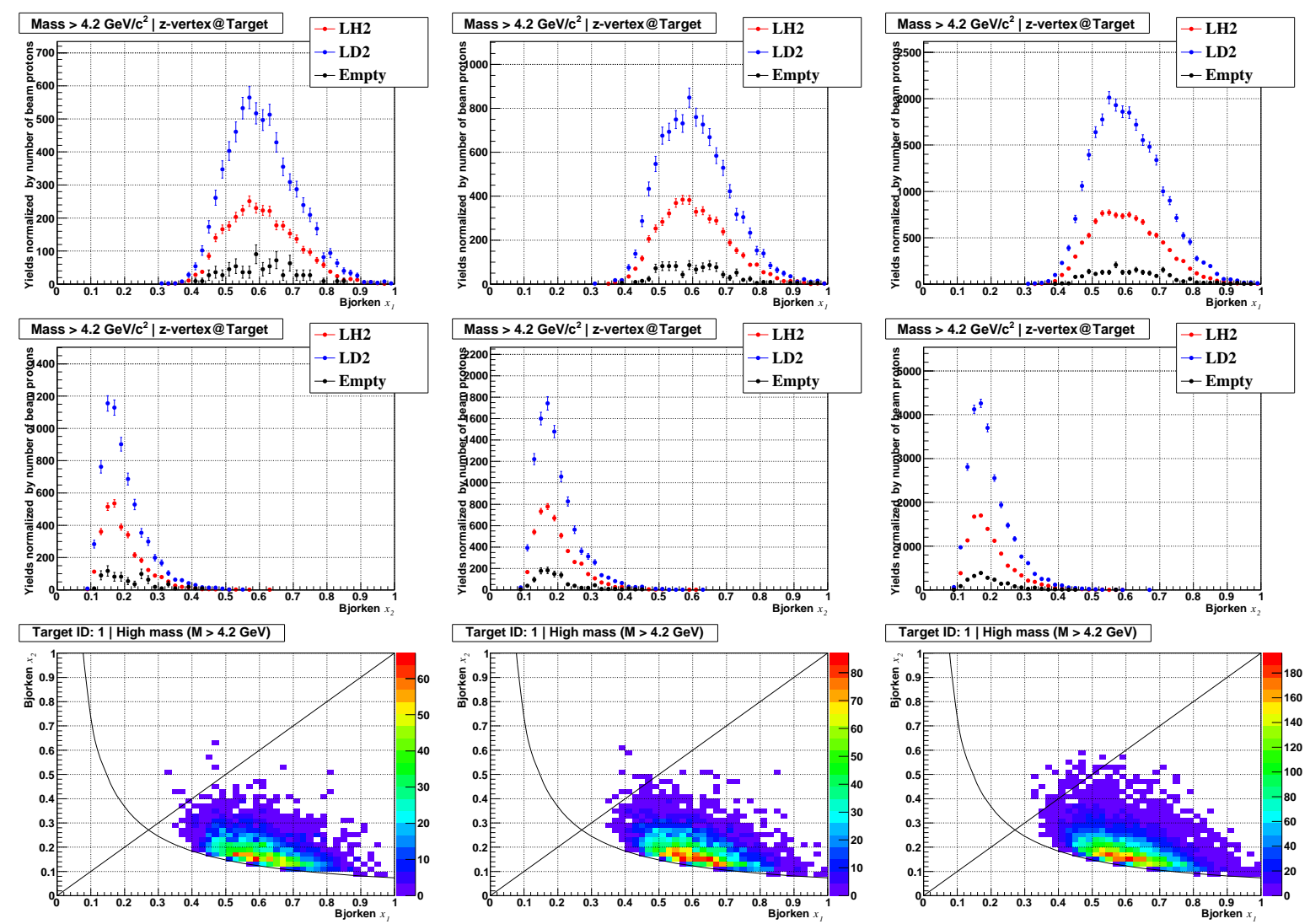

Figure 5.16: Distributions of $x_{1}$ (top), $x_{2}$ (middle) and $x_{2}$ vs $x_{1}$ (bottom) for Roadset 57, 62 and 67 from left to right. A straight line of $x_{2}=x_{1}$ and a hyperbolic curve of $M=4.2 \mathrm{GeV} / \mathrm{c}^{2}$ are drawn on the $x_{2}$ vs $x_{1}$ figure. 

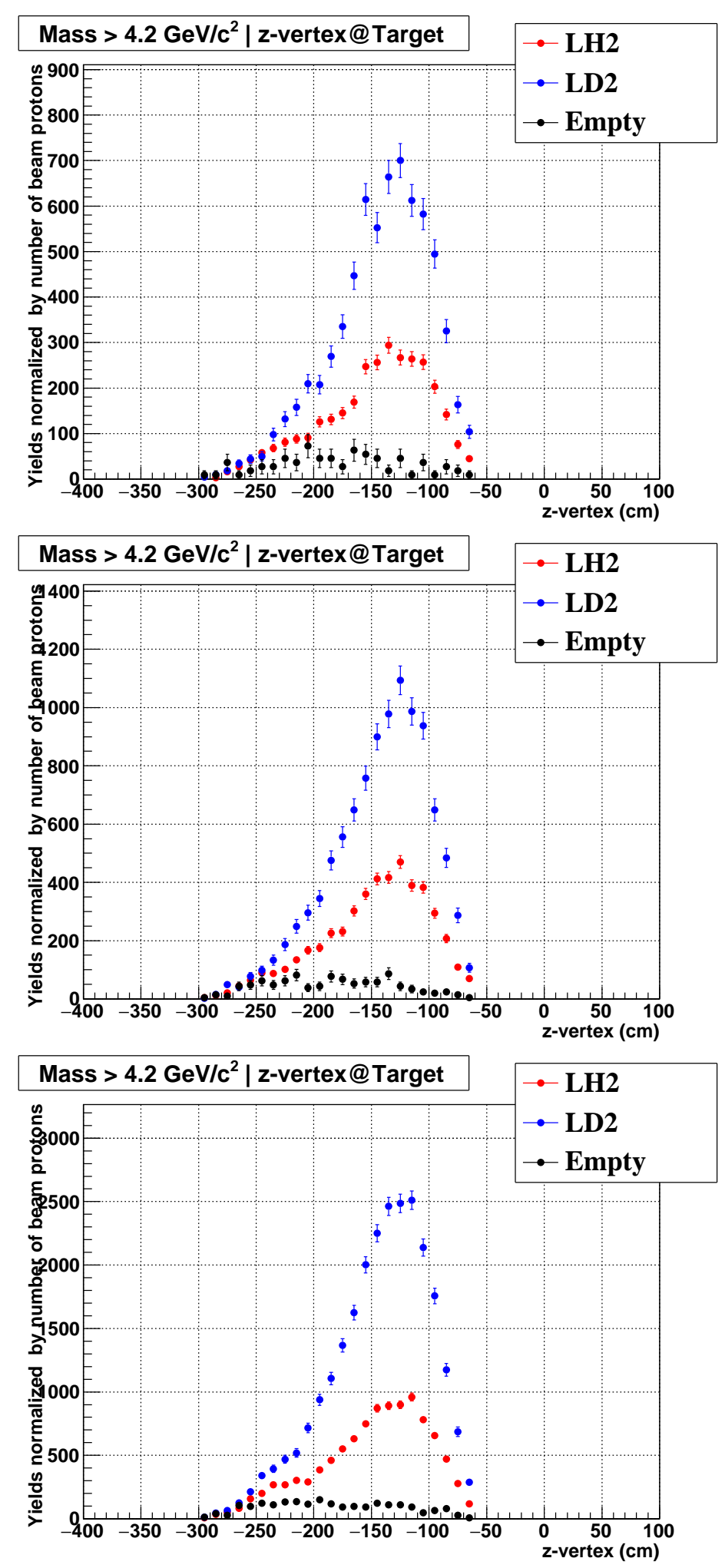

Figure 5.17: Distribution of $z$-vertex for Roadset 57, 62 and 67 data. The blue points show yield of LD2 events, the red points show that of LH2 events, and black points show that of empty target events. 


\subsubsection{Hit distribution on chamber plane}

Hit distributions as a function of element ID (cell ID) on chamber planes are shown in Fig. 5.18. These are the results by raw data of one run. These figures are useful to see the general trend of hit distributions on the chamber plane. No selections are applied. Outer edges of plane have more hits than center of the plane because of the magnets.

\subsubsection{Occupancy of chamber plane}

Figure 5.19 shows the occupancy of chamber plane. These are the results by raw data of one run. The definition of occupancy here is the number of hits divided by the number of cells of the chamber plane in one trigger event. Roughly $5-10 \%$ of cells usually have a hit in one trigger event.

\subsubsection{Track position on chamber plane}

2D histograms of track positions at each X plane of drift chambers are shown in Fig. 5.20 . These are the results of data after all selections are applied. The tracks usually penetrate around the center at Station 1 drift chamber, and travel towards the outer edges at Station 2 and 3 drift chambers because of magnets. 

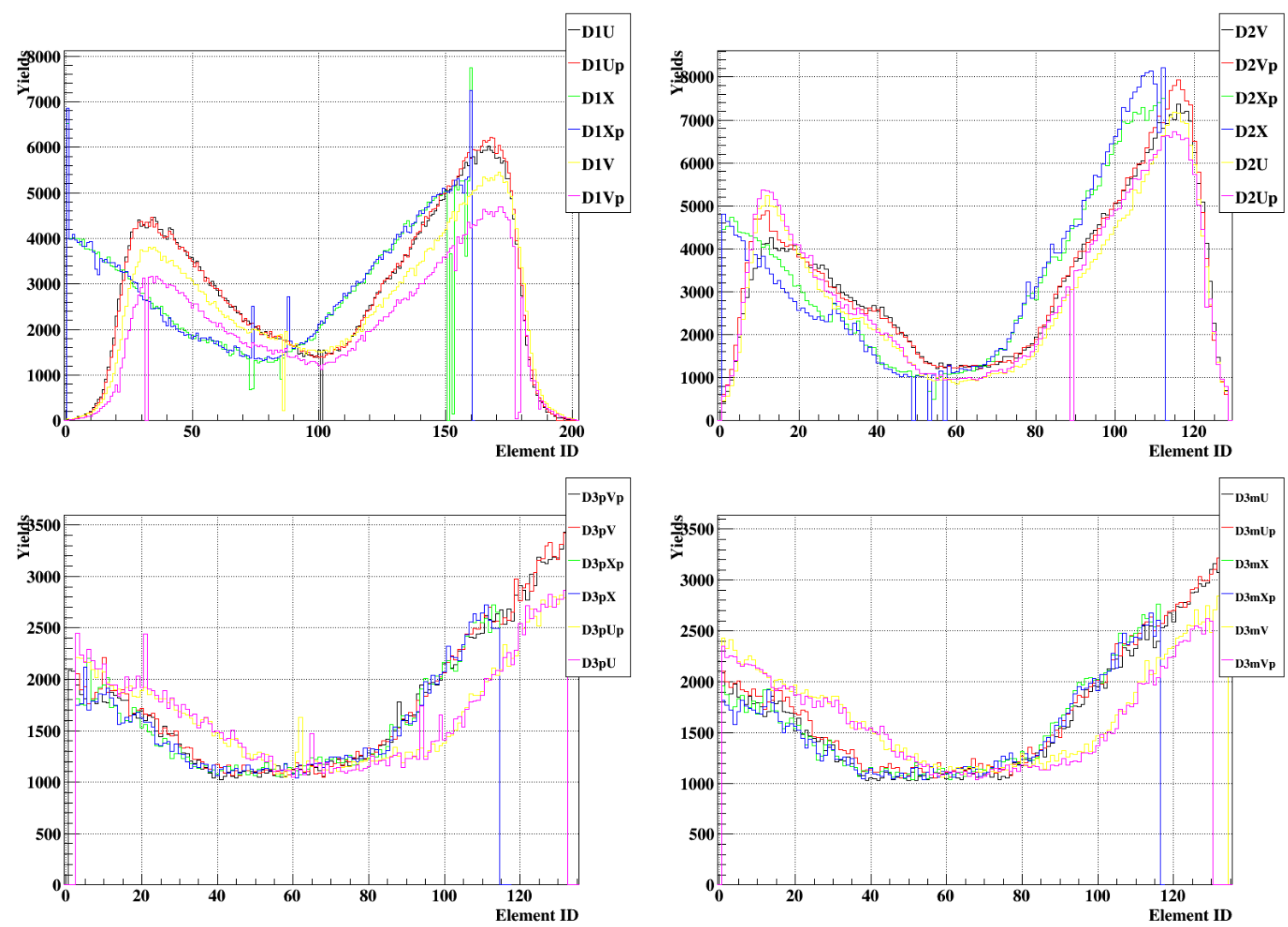

Figure 5.18: Hit distributions as a function of element ID (cell ID) on chamber planes. These are the results by raw data of one run. These top-left, top-right, bottom-left and bottom-right figures show the hit distributions of D1, D2, D3 plus and D3 minus, respectively. 

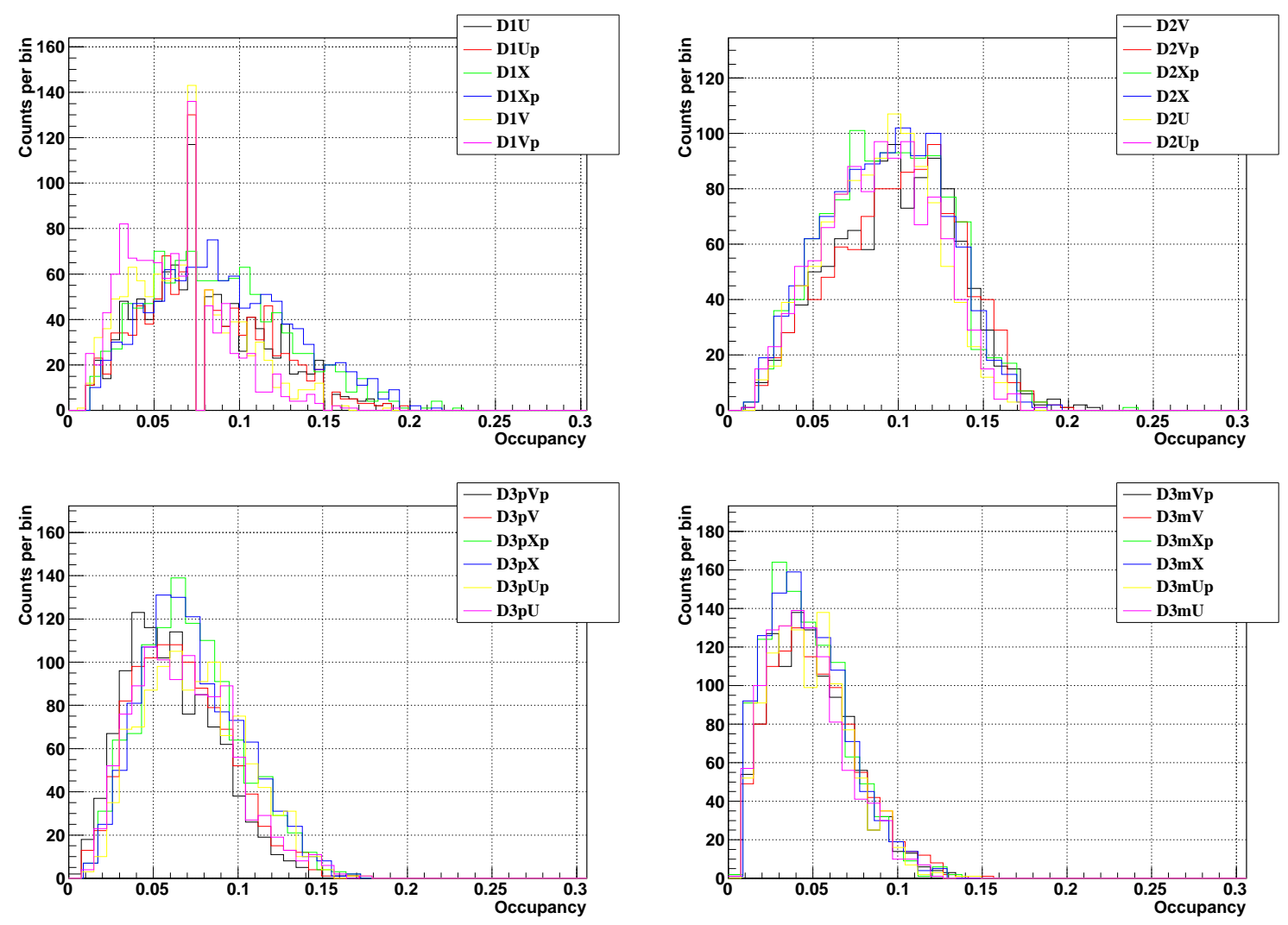

Figure 5.19: Occupancy of chamber plane. Those top-left, top-right, bottom-left and bottom-right figures show the occupancy distributions of D1, D2, D3 plus and D3 minus, respectively. 
D1

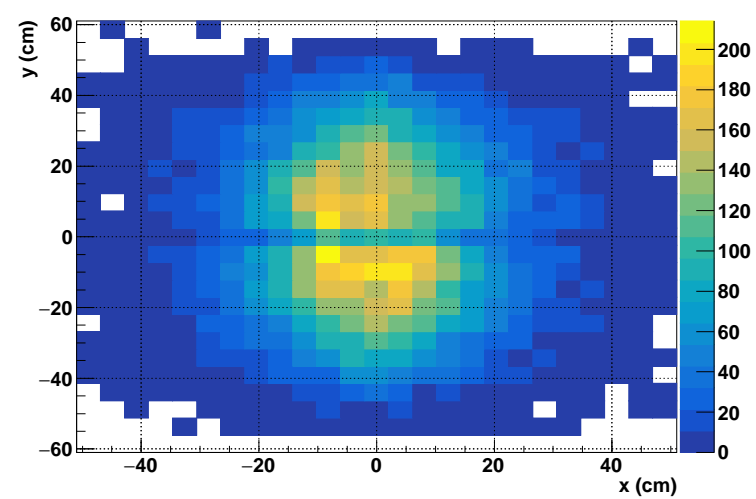

D3p

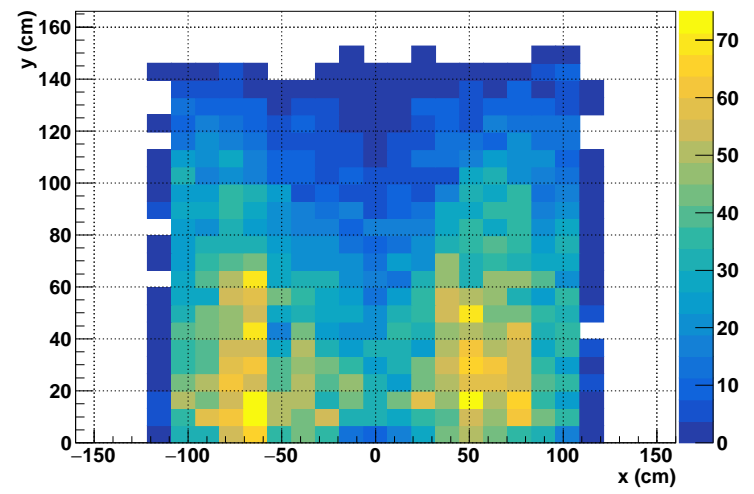

D2

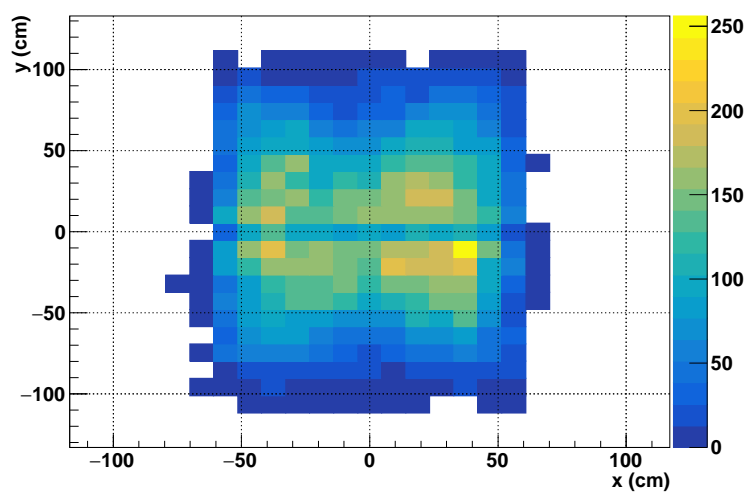

D3m

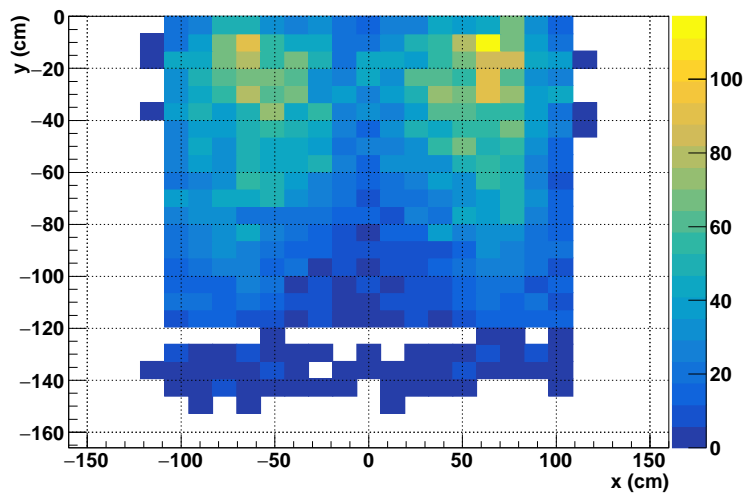

Figure 5.20: 2D histograms of track positions at each $\mathrm{X}$ plane of drift chambers. These are the results of data after all selections are applied. These top-left, top-right, bottomleft and bottom-right figures show the 2D histograms of track positions of D1X, D2X, D3pX and D3mX, respectively. 


\subsection{Drift chamber performance}

The drift chambers are the key component in the SeaQuest spectrometer. The details of its performance are described in this section.

\subsubsection{R-T curve}

When a charged particle traverses in a drift chamber, it hits electrons out of gas atoms in the chamber. The electrons then drift to the sense wire, and the drift chamber outputs the drift time information. It is needed to convert the time information to distance information in order to find the hit position. The function of the conversion is called "R-T curve". It is an important calibration for operating drift chambers.

In this calibration process, drift distance information is given by track reconstruction. Corresponding TDC time was already given by the measurement. 2D distribution of the drift distance vs TDC time is made. An R-T curve is then obtained by fitting this $2 \mathrm{D}$ distribution.

Iteration analysis is done to improve the R-T curve: Obtained R-T curve is used in the tracking in the next iteration step, and then improved $\mathrm{R}-\mathrm{T}$ curve is obtained using the result of the second reconstruction. This iteration process continues until the R$\mathrm{T}$ shape becomes stable. It was done four times in our analysis. Since no R-T curve information exists for the first track reconstruction, a simple function or a function given by simulations are usually used for the first try.

There are two drift directions to a sense wire; positive $x$ direction and negative $x$ direction. An R-T curve is first obtained for the both side and then they are merged as shown in Fig. $5.2 \pi$ after it is confirmed that the R-T curve for the both side is symmetric about $R=0$. Figure $5.2 \mathrm{~J}$ shows $2 \mathrm{D}$ plots of drift distance vs measured TDC time for the $\mathrm{U}$ plane of the Station 3 minus drift chamber as an example. Also, an R-T curve obtained by fitting is shown in black on the plot. These four figures show the result of the first to fourth iteration from left to right and top to bottom. The horizontal axis indicates measured TDC time. A larger value in TDC time indicates smaller drift time since the SeaQuest TDC works as a common-stop TDC. The distribution became sharper as the iteration analysis is repeated. This fact indicates that the $\mathrm{R}-\mathrm{T}$ curve becomes close to the chamber's intrinsic R-T curve.

Figure 5.22 shows residuals as a function of measured TDC time in the first to the fourth iteration analysis for the $\mathrm{U}$ plane of the Station 3 minus drift chamber. The 

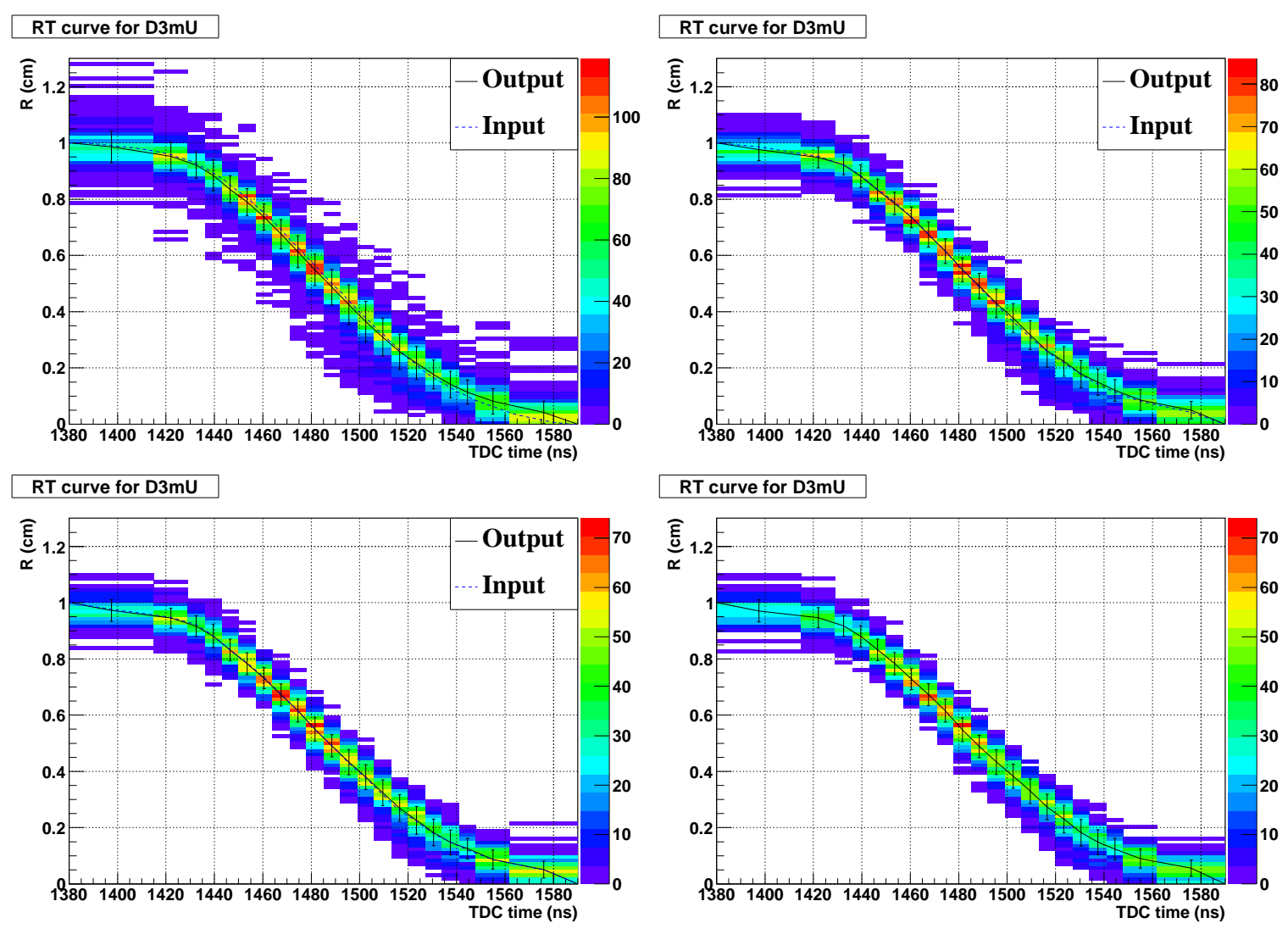

Figure 5.21: Drift distance (R) as a function of measured TDC time in the first to the fourth iteration analysis for the $\mathrm{U}$ plane of the Station 3 drift chamber: top-left is the first iteration's result, top-right the second one, bottom-left the third one, and bottom-right the fourth one. The distribution became sharper as the iteration analysis is repeated. 

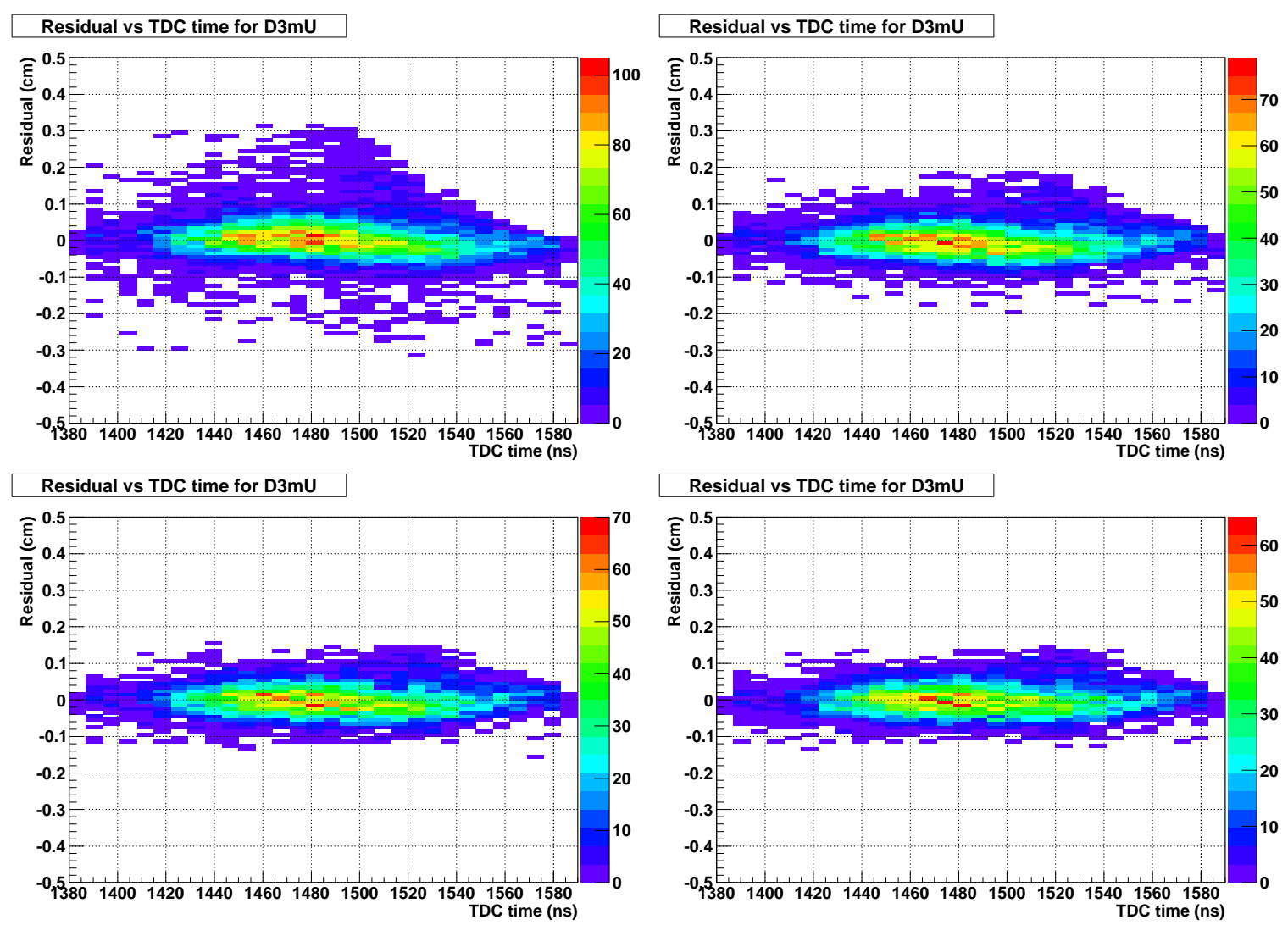

Figure 5.22: Residuals as a function of measured TDC time in the first to the fourth iteration analysis for the $U$ plane of the Station 3 drift chamber from left to right and top to bottom. The definition of the residual is Eq. 5.5. The distribution becomes sharper and symmetric about residual $=0$ as the iteration analysis is repeated.

definition of the residual is:

$$
(\text { residual })=r_{\mathrm{drift}}-f\left(t_{\mathrm{drift}}\right)
$$

where $r_{\text {drift }}$ is the drift distance obtained by the track reconstruction, $f\left(t_{\text {drift }}\right)$ is the R-Tcurve, and $t_{\text {drift }}$ is the measured TDC time. The distribution becomes symmetric about residual $=0$ as the iteration is repeated. This fact indicates also that the $\mathrm{R}$ - $\mathrm{T}$ curve becomes close to the chamber's intrinsic R-T curve.

Figure 5.23 shows the spacial resolution as a function of measured TDC time in the first to the fourth iteration analysis for the the Station 3 minus drift chamber. The resolution is the RMS of the residual distribution in each TDC bin. The resolution became better as the iteration analysis is repeated. Figure 5.24 shows the resolution as a function of measured TDC time of the fourth iteration analysis for all the drift chamber. 
kTracker uses hit information of all the drift chamber planes (18 planes in total $\left.{ }^{\mathbb{W}}\right)$ to select more probable track and to calculate $\chi^{2}$ of tracks in the tracking algorithm. Track reconstruction is done with higher accuracy by using the hit information of all planes. On the other hand, when the spacial resolution of a chamber plane is calculated, the hit information of the plane is commonly ignored and the hit information of the other 17 planes are used for the track reconstruction in order to exclude the bias by the plane. SeaQuest didn't take this method, but used the track reconstructed by all planes' hit information to obtain the position resolution of chamber planes. In this case, the position resolution of all the planes can be calculated at once while 24 times of analysis are needed for the usual method. Then the calculated spacial resolution is corrected in order to obtain its intrinsic resolution. The correction factors are 1.1 for Station 1, 3 plus and 3 minus drift chambers and 1.05 for Station 2 drift chamber [40]. They are multiplied to the calculated spacial resolution. Table 5.13 summarizes the correction factors, obtained resolution, and corrected resolution for all the drift chambers.

\begin{tabular}{llll}
\hline \hline Drift Chamber & Correction factor & $\begin{array}{l}\text { Obtained } \\
\text { resolution }(\mu \mathrm{m})\end{array}$ & $\begin{array}{l}\text { Corrected } \\
\text { resolution }(\mu \mathrm{m})\end{array}$ \\
\hline D1 & 1.1 & 300 & 330 \\
D2 & 1.05 & 400 & 420 \\
D3 plus & 1.1 & 400 & 440 \\
D3 minus & 1.1 & 450 & 500 \\
\hline
\end{tabular}

Table 5.13: Correction factors, obtained resolution, and corrected resolution for all the drift chambers

The resolution of D3 minus is less than $500 \mu \mathrm{m}$. This is larger than designed value, but still acceptable because the contribution of the chamber resolution to the muon pair mass resolution is small.

\footnotetext{
${ }^{1}$ There are four drift chambers in the SeaQuest spectrometer, and each chamber has six planes, thus there are 24 planes in total. But since the Station 3 plus covers upper area at Station 3 and Station 3 minus covers lower area there, one track can penetrate 18 planes at a maximum.
} 

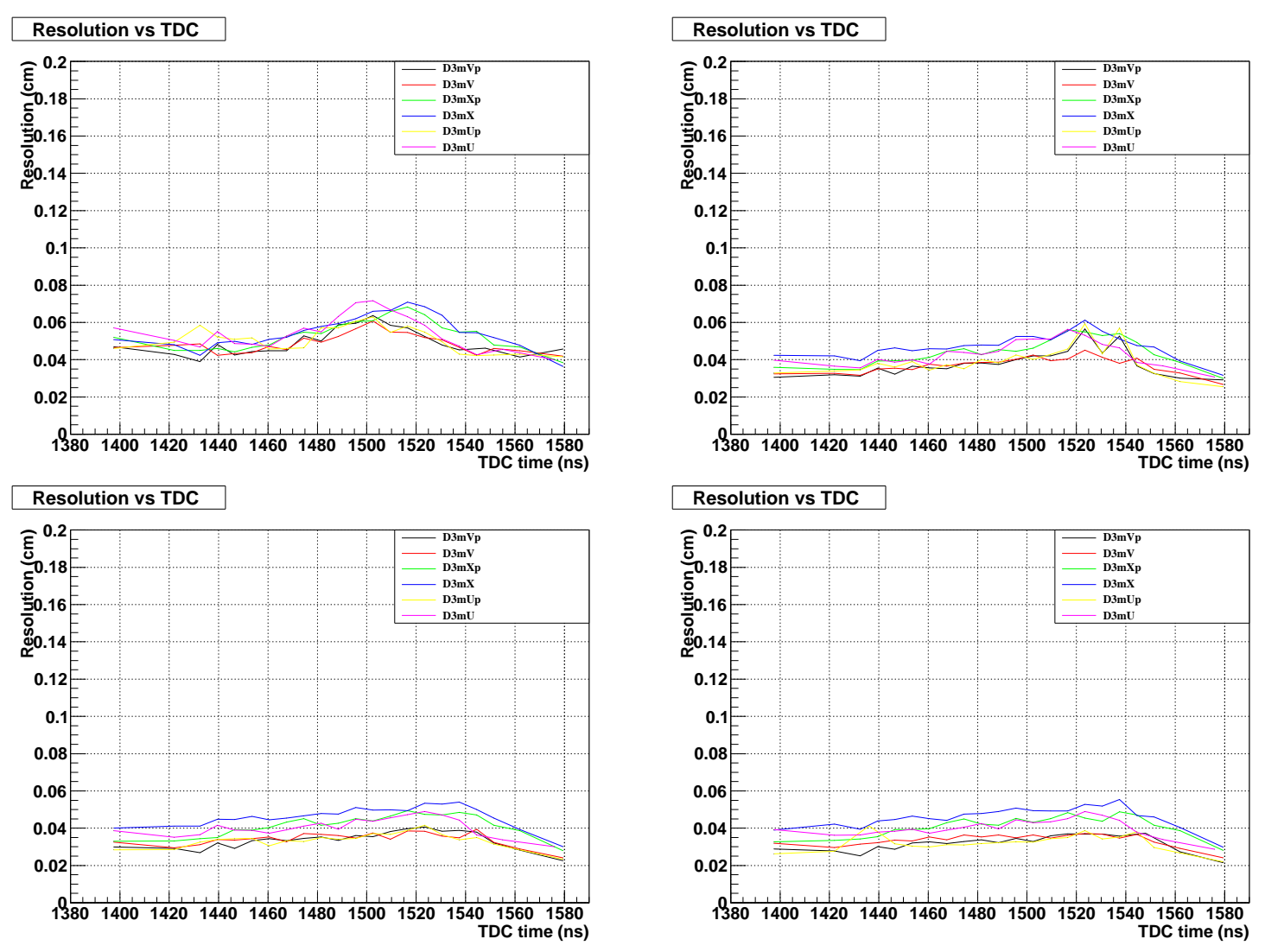

Figure 5.23: Resolution vs TDC time in the first to the fourth iteration analysis for Station 3 minus drift chamber. The resolution became better as the iteration is repeated. 

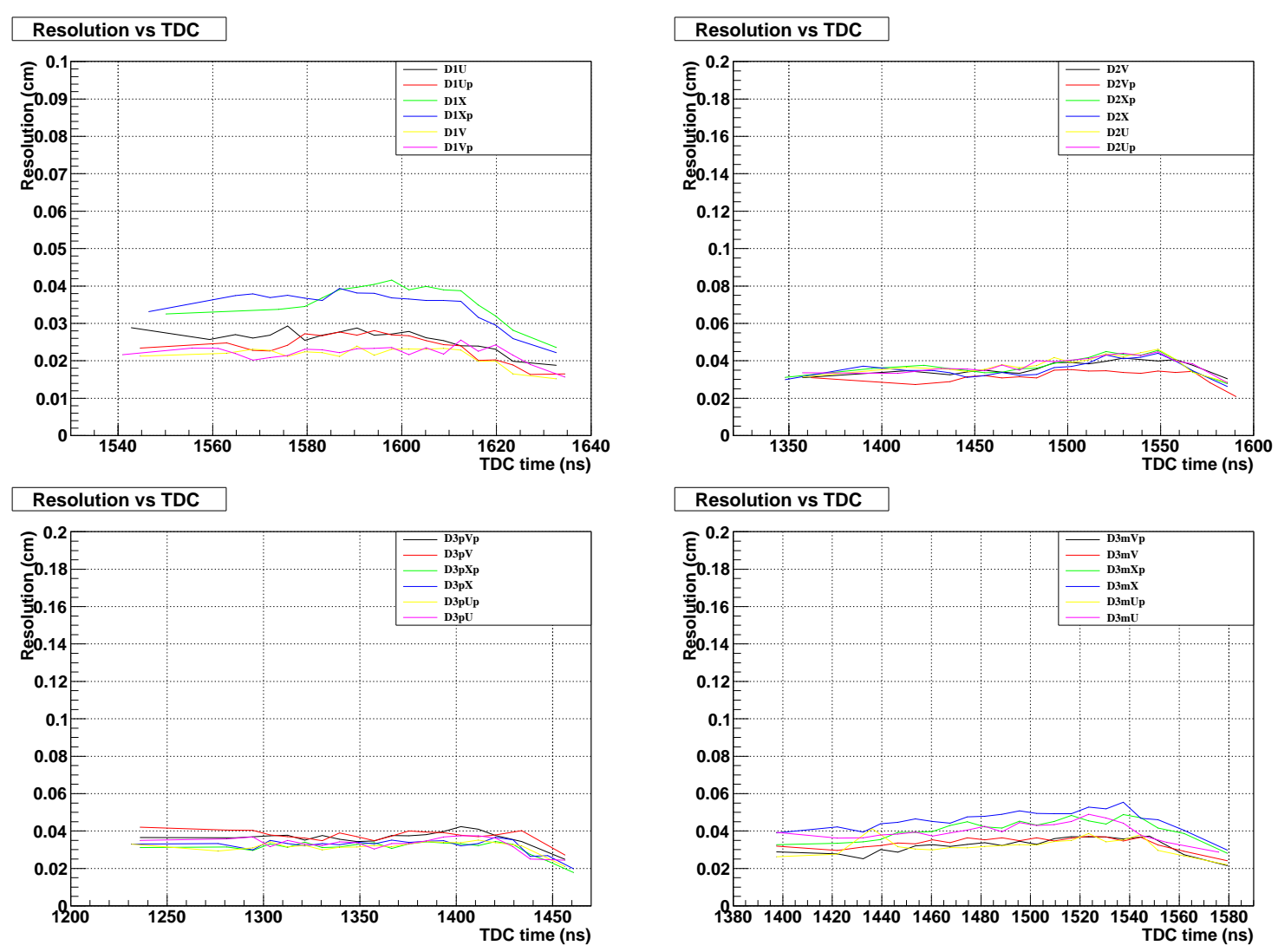

Figure 5.24: Results of resolution vs TDC time of the fourth iteration analysis for Station 1 (top-left), Station 2 (top-right), Station 3 plus (bottom-left) and Station 3 minus (bottom right) drift chambers. 


\subsubsection{Chamber efficiency}

In addition to the spacial resolution, the detection efficiency is an important characteristic of the chamber performance. "Good" tracks, which passed the following extra tight cuts, are used to calculate detection efficiency of the drift chambers:

- Reduced $\chi^{2}$ of track reconstruction is less than 2.0

- number of hits associated with the track $\geq 17$.

Two kinds of tracks in the selected tracks are used to calculate efficiency of a plane: "efficient track" which has 18 associated hits on it, and "in-efficient track" which doesn't have an associated hit on the plane and does have it on the other 17 planes. If the track position is close to the dead or noisy wires, the track is not used for the efficiency calculation in order to ignore the effects of them. Using those tracks, the plane efficiency is evaluated as:

$$
\epsilon=\frac{N_{\text {eff }}}{N_{\text {eff }}+N_{\text {ineff }}}
$$

where $N_{\text {eff }}$ is the number of efficient tracks and $N_{\text {ineff }}$ is the number of in-efficient tracks. Table 5.14 summarizes the chamber efficiency during the physics run. Each chamber plane is equally divided into three sections in terms of $x$-axis: right $(-W / 2 \leq x \leq-W / 6)$, center $(-W / 6<x<W / 6)$, and left $(W / 6 \leq x \leq W / 2)$ where $W$ is the width of each chamber plane. Chamber plane efficiency is then calculated for each section and each chamber plane. The results show that the efficiencies at edges are less than that at the center. This is because of the after-pulse removal in track reconstruction process. Due to the settings of the magnetic field, the hit rate at the edge of the drift chambers is higher than that at the center. Because of the high hit rate, extra hit (background muons, muons which come from the other RF bucket for example) can be observed earlier than true hit, and it is discarded by the after-pulse removal. This can happen at the edge of the chamber plane with higher probability than at the center. 


\begin{tabular}{llll}
\hline \hline Plane & Edge (Left) & Center & Edge (Right) \\
\hline D1U & $90.0 \pm 1.6 \%$ & $98.0 \pm 0.0 \%$ & $92.9 \pm 0.8 \%$ \\
D1Up & $88.1 \pm 1.7 \%$ & $98.3 \pm 0.0 \%$ & $91.9 \pm 0.8 \%$ \\
D1X & $81.2 \pm 2.0 \%$ & $97.3 \pm 0.1 \%$ & $87.8 \pm 1.0 \%$ \\
D1Xp & $82.4 \pm 2.0 \%$ & $97.5 \pm 0.1 \%$ & $89.5 \pm 0.9 \%$ \\
D1V & $92.2 \pm 2.1 \%$ & $98.7 \pm 0.0 \%$ & $94.4 \pm 0.7 \%$ \\
D1Vp & $92.6 \pm 1.3 \%$ & $98.4 \pm 0.0 \%$ & $93.7 \pm 0.8 \%$ \\
\hline D2V & $90.6 \pm 0.6 \%$ & $95.6 \pm 0.1 \%$ & $93.5 \pm 0.2 \%$ \\
D2Vp & $91.7 \pm 0.6 \%$ & $96.4 \pm 0.1 \%$ & $94.6 \pm 0.2 \%$ \\
D2Xp & $89.3 \pm 0.3 \%$ & $91.2 \pm 0.3 \%$ & $90.6 \pm 0.2 \%$ \\
D2X & $90.4 \pm 0.2 \%$ & $91.9 \pm 0.5 \%$ & $91.6 \pm 0.2 \%$ \\
D2U & $90.8 \pm 0.3 \%$ & $95.4 \pm 0.1 \%$ & $93.7 \pm 0.2 \%$ \\
D2Up & $90.6 \pm 0.3 \%$ & $95.9 \pm 0.1 \%$ & $94.4 \pm 0.2 \%$ \\
\hline D3pVp & $89.3 \pm 0.1 \%$ & $91.3 \pm 0.5 \%$ & $88.7 \pm 0.4 \%$ \\
D3pV & $90.8 \pm 0.1 \%$ & $92.6 \pm 0.4 \%$ & $89.4 \pm 0.4 \%$ \\
D3pXp & $91.5 \pm 0.1 \%$ & $93.1 \pm 0.5 \%$ & $91.5 \pm 0.2 \%$ \\
D3pX & $91.3 \pm 0.1 \%$ & $92.9 \pm 0.5 \%$ & $92.2 \pm 0.2 \%$ \\
D3pUp & $90.4 \pm 0.5 \%$ & $93.1 \pm 0.5 \%$ & $92.4 \pm 0.2 \%$ \\
D3pU & $90.3 \pm 0.3 \%$ & $92.4 \pm 0.4 \%$ & $90.9 \pm 0.2 \%$ \\
\hline D3mVp & $94.5 \pm 0.5 \%$ & $96.1 \pm 0.3 \%$ & $94.8 \pm 0.1 \%$ \\
D3mV & $93.8 \pm 0.5 \%$ & $97.0 \pm 0.3 \%$ & $95.4 \pm 0.1 \%$ \\
D3mXp & $94.3 \pm 0.1 \%$ & $95.4 \pm 0.4 \%$ & $94.3 \pm 0.1 \%$ \\
D3mX & $94.0 \pm 0.1 \%$ & $95.3 \pm 0.4 \%$ & $94.6 \pm 0.1 \%$ \\
D3mUp & $94.0 \pm 0.2 \%$ & $95.5 \pm 0.4 \%$ & $94.9 \pm 0.3 \%$ \\
D3mU & $93.3 \pm 0.2 \%$ & $94.2 \pm 0.4 \%$ & $93.7 \pm 0.3 \%$ \\
\hline
\end{tabular}

Table 5.14: Drift chamber plane efficiency 


\subsection{Extraction of Cross Section Ratio}

The procedure to extract the cross section ratio from the hydrogen target data and the deuterium target data is described in this section.

\subsubsection{Number of proton per RF bucket}

The first step to extract the cross section ratio is to have a correct number of the beam protons used in the measurement. The number of beam protons in each RF bucket measured by QIE has an offset value, called "pedestal". Using NIM3 event that is a random trigger (NIM3 trigger), the QIE pedestal is calculated by selecting events whose G2SEM value is equal to zero, which means no beam arrived. Figure 5.25 shows the QIE values of such events as a function of spill ID. The pedestal is changed during Roadset 62 due to QIE hardware work. The average pedestal value is 36.2 for spill ID 300000 to 450000, and 32.6 for spill ID 450000 to 680000 (Table 5.15).

\begin{tabular}{ll}
\hline \hline Spill range & Pedestal value \\
\hline $300000-450000$ & 36.2 \\
$450000-680000$ & 32.6 \\
\hline
\end{tabular}

Table 5.15: Averaged pedestal value for each spill range.

In the database, the measured QIE value in the triggering RF bucket is kept, as well as the value for the 16 preceding and 16 following buckets. They are named as "RF-16" through " $\mathrm{RF}+16$ ". The value in terms of protons is calculated by:

$$
Q_{\mathrm{RF}+\mathrm{xx}}^{\mathrm{pc}} \cdot P_{\mathrm{G} 2 \mathrm{SEM}} / Q_{\mathrm{sum}}^{\mathrm{pc}},
$$

where $Q_{\mathrm{RF}+\mathrm{xx}}^{\mathrm{pc}}||_{-16 \leq X X \leq+16}$ is the $\mathrm{QIE}$ value of the $\mathrm{RF}+\mathrm{XX}, P_{\mathrm{G} 2 \mathrm{SEM}}$ is the absolute numbers of protons measured by G2SEM for the spill, $Q_{\mathrm{sum}}^{\mathrm{pc}}$ is the sum of QIE value for the spill, and the subscript pc stands for pedestal corrected. The pedestal correction is done as:

$$
\begin{aligned}
Q_{\mathrm{RF}+\mathrm{xx}}^{\mathrm{pc}} & =Q_{\mathrm{RF}+\mathrm{xx}}-p \\
Q_{\mathrm{sum}}^{\mathrm{pc}} & =Q_{\mathrm{sum}}-p * 588 * 360000,
\end{aligned}
$$

where $p$ is pedestal value, and its coefficient in the second equation means that the proton beam goes around the Main Injector ring 360000 times in one spill and 588 buckets are in one ring at maximum. 

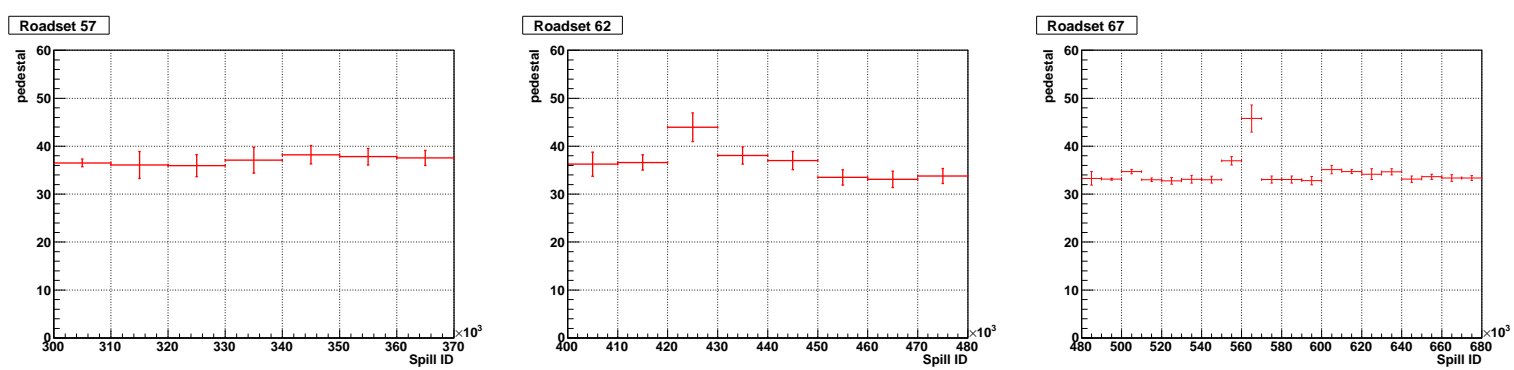

Figure 5.25: Pedestal of the Cerenkov beam counter as a function of spill ID. The results of Roadset 57, 62 and 67 data are shown from left to right.

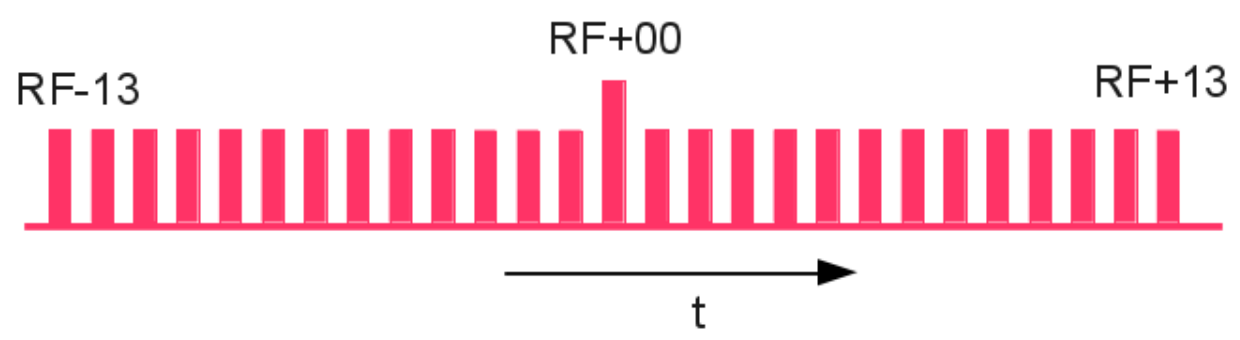

Figure 5.26: The RF structure around the $R F+00$ is shown. The preceding $R F$ buckets are named as $\mathrm{RF}-X X$ and the following $\mathrm{RF}$ buckets are named as $\mathrm{RF}+X X$.

\subsection{2 "Trigger intensity" and "Chamber intensity"}

Now, two different measures of beam intensity are defined for several analysis purposes: "Trigger intensity" and "Chamber intensity". The trigger intensity is just the number of protons in the triggering $\mathrm{RF}$ bucket $(\mathrm{RF}+00)$ :

$$
I_{\text {trigger }}=Q_{\mathrm{RF}+00}^{\mathrm{pc}} \cdot P_{\mathrm{G} 2 \mathrm{SEM}} / Q_{\mathrm{sum}}^{\mathrm{pc}} \cdot
$$

Simply using the value of $\mathrm{RF}+00$ is not sufficient to describe the effects of intensity on the chambers because the in-time window of the drift chambers are long enough to cover multiple RF buckets. The chamber in-time window is roughly 100-200 ns depending on the station, while the RF is spaced by roughly 19 ns as shown in Fig. 4.3]. The chamber R-T curves are used to calculate the probability that a track from a preceding or following $\mathrm{RF}$ bucket is inside the in-time window of the chamber. The RF structure around the $\mathrm{RF}+00$ is shown in Fig. 5.26. The preceding $\mathrm{RF}$ buckets are named as $\mathrm{RF}-X X$ and the following $\mathrm{RF}$ buckets are named as $\mathrm{RF}+X X$.

For example, for a track from $\mathrm{RF}+03$, its drift time has an offset of about $57 \mathrm{~ns}(=19$ ns $\times 3$ ). Thus, the hits from tracks with a drift time of $T_{\mathrm{Max}}-57$ or less will come into the 
chamber in-time window, where $T_{\mathrm{Max}}$ is the maximum drift time. From the R-T curve, the drift distance, $d$, that corresponds to the drift time $\left(T_{\mathrm{Max}}-57\right) \mathrm{ns}$ is given. If it is assumed that the hit position distribution in a cell is uniform, the probability that the track from $\mathrm{RF}+03$ comes into the chamber in-time window is $d / d_{\text {Max }}$, where $d_{\text {Max }}$ is the maximum drift distance. For the preceding buckets, the probability can be calculated in the similar way. The weight for each RF bucket is calculated as follows by taking into account the fact that the SeaQuest TDC is a common stop:

$$
\begin{aligned}
& \left.w_{i}\right|_{i>0}=f\left(T_{1}+19 \cdot i\right) / d_{\mathrm{Max}} \\
& \left.w_{i}\right|_{i<0}=1-f\left(T_{0}-19 \cdot i\right) / d_{\mathrm{Max}}
\end{aligned}
$$

where $T_{0}$ and $T_{1}$ are the maximum and the minimum value of TDC time (right edge and left edge of $x$-axis of Fig. 5.27 ), and $f(t)$ is the R-T curve like Fig. 5.27 . Averaged R-T curve was used to obtain the weights. The weights are listed in Table 5.16].

The chamber intensity is based on the weighted average over buckets $\mathrm{RF}-13$ through $\mathrm{RF}+13$. It is defined as:

$$
I_{\text {chamber }}=\frac{\sum_{i=-13}^{13} w_{i} Q_{\mathrm{RF}+\mathrm{i}}^{\mathrm{pc}}}{\sum_{i=-13}^{13} w_{i}} P_{\mathrm{G} 2 \mathrm{SEM}} / Q_{\mathrm{sum}}^{\mathrm{pc}},
$$

where $w_{i}$ is the weight of $\mathrm{RF}+i$. Figure 527 shows a 2D histogram of trigger intensity vs chamber intensity, an 1D histogram of the two intensities, and the ratio of those (chamber intensity divided by trigger intensity). The trigger intensity distribution has more events at higher intensity region. This is because the chamber intensity is the weighted average of the beam intensity of preceding and following RF buckets (from RF-13 to RF+13). 

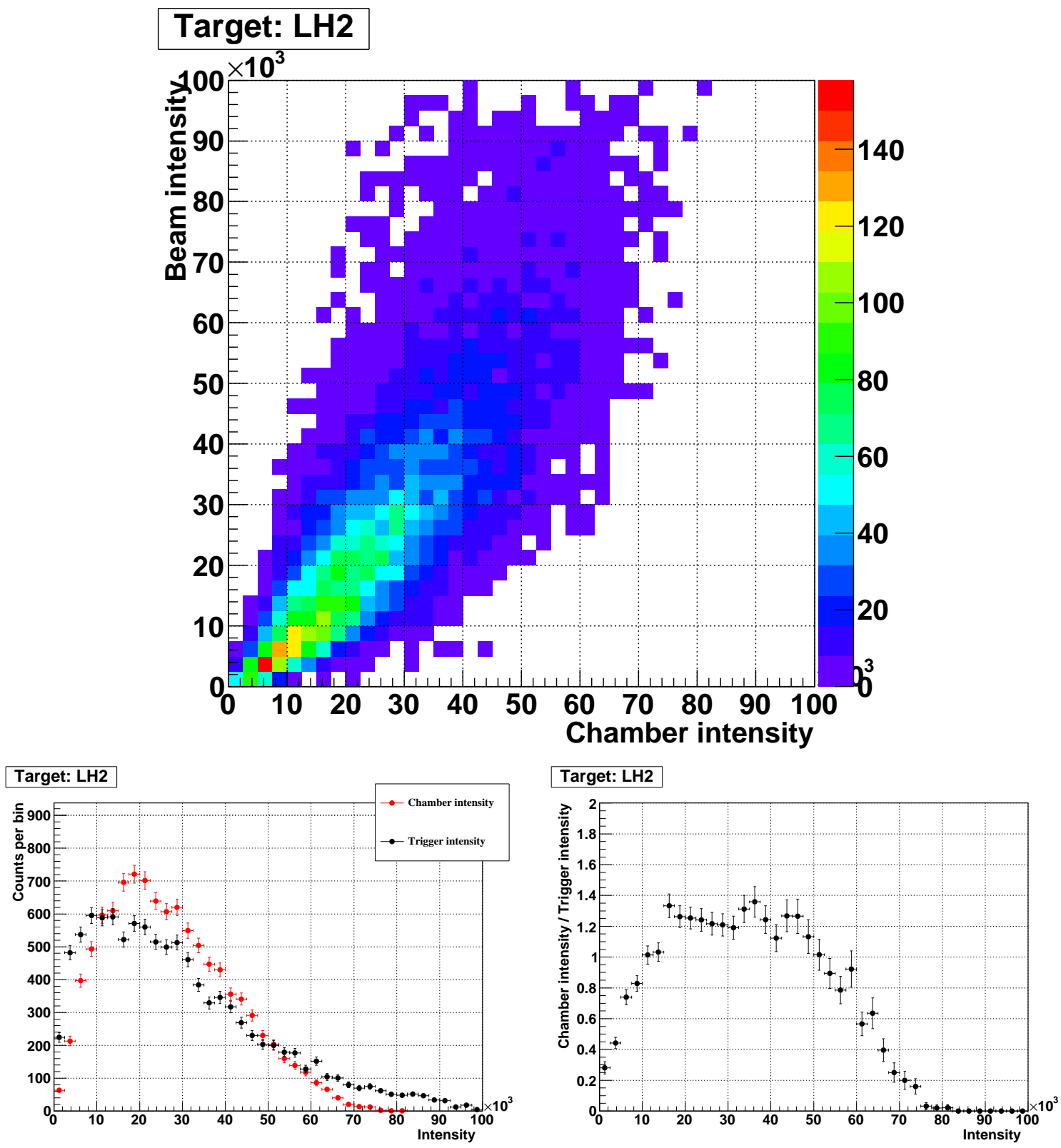

Figure 5.27: 2D histogram of trigger intensity vs chamber intensity (top), 1D histogram of the two intensities (bottom-left), and the ratio of those (chamber intensity over trigger intensity) (bottom-right). 


\begin{tabular}{ll}
\hline \hline RF ID & Probability \\
\hline-13 & 0.000814 \\
-12 & 0.002866 \\
-11 & 0.005970 \\
-10 & 0.012126 \\
-09 & 0.030086 \\
-08 & 0.077726 \\
-07 & 0.159447 \\
-06 & 0.259933 \\
-05 & 0.367189 \\
-04 & 0.488159 \\
-03 & 0.678969 \\
-02 & 0.847788 \\
-01 & 0.956475 \\
+00 & 1.000000 \\
+01 & 0.989174 \\
+02 & 0.897678 \\
+03 & 0.767829 \\
+04 & 0.647167 \\
+05 & 0.533895 \\
+06 & 0.448849 \\
+07 & 0.356435 \\
+08 & 0.263693 \\
+09 & 0.177965 \\
+10 & 0.108505 \\
+11 & 0.054010 \\
+12 & 0.019219 \\
+13 & 0.003983 \\
\hline &
\end{tabular}

Table 5.16: Average weight for each RF bucket based on chamber R-T curves. 

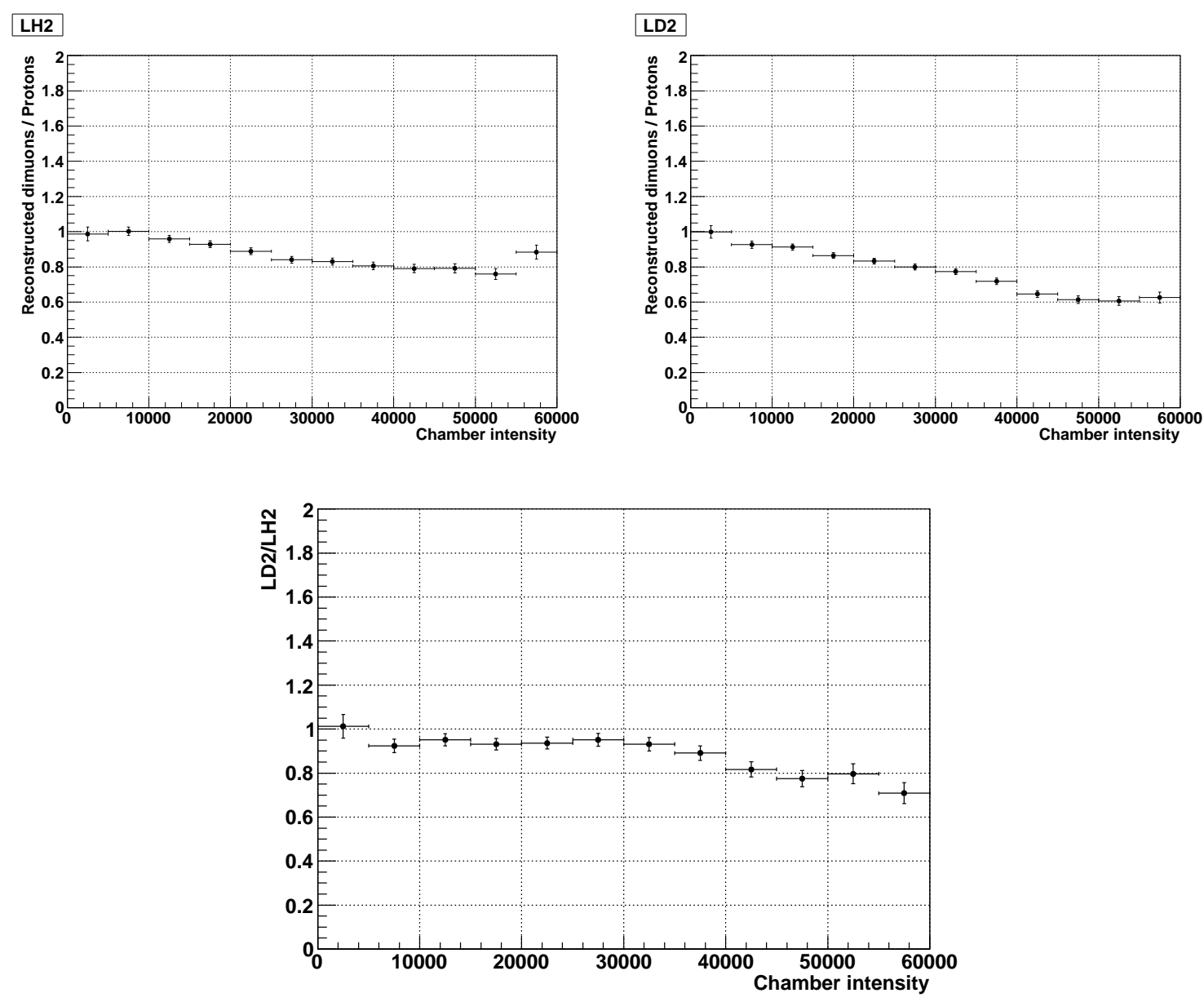

Figure 5.28: The yield of Drell-Yan dimuon per unit of trigger intensity is shown as a function of chamber intensity for LH2 target (top-left) and LD2 target (top-right). The ratio of the dimuon yields between LD2 and LH2 is shown at bottom. These are the results using data.

\subsubsection{Intensity dependence correction}

The intensity dependence correction is made to remove the effect of any inefficiency that occurs as a function of intensity. Figure 5.28 shows the yield of Drell-Yan dimuon per unit of trigger intensity as a function of chamber intensity for LH2 target (top-left) and LD2 target (top-right). The drop in the yield of dimuon as the chamber intensity increases can be seen in the figure. The ratio of dimuons between LD2 and LH2 is also shown in Fig. 5.28. It should be noted in these figures that the LD2 events have larger inefficiency at large chamber intensity than the LH2 events, which could affect the cross section ratio.

Two sets of Monte Carlo simulation are used to correct for the intensity dependence 
instead of data because the statistics of data is not high enough for this study [38, [3.]. The first set, the "clean set", is simply GMC Drell-Yan data. The second set, the "messy set", is the same GMC events with the hits from events from data triggered by the random trigger (NIM3 trigger). Both sets are then passed to the track reconstruction. The tracking efficiency can be calculated by dividing the number of good dimuons in the messy set by the number of good dimuons in the clean set. The efficiency as a function of the chamber intensity is obtained for both hydrogen and deuterium target, and also for each $x_{2}$ bin. They are then fitted by exponential curve, $\exp \left(p_{0} I\right)$, as can be seen in Fig. 5.2.9 for hydrogen events, and Fig. 5.30 for deuterium events. Also two more exponential curves are drawn on the figures. These curves correspond to $p_{0}$ shifted by $+1 \sigma$ or $-1 \sigma$ from the original value. The detail values are listed in Table 5.77. Each dimuon event is weighted by $1 / \exp \left(p_{0} I\right)$ where $I$ is the chamber intensity, to correct for the intensity dependence. The weight, $1 / \exp \left(p_{0} I\right)$, as a function of the chamber intensity is also shown in Fig. 5.31 and Fig. 5.32. Three curves are also shown in the same way.

\begin{tabular}{lll}
\hline \hline$x_{2}$ bin & LH2 target & LD2 target \\
\hline 1 & $-1.3700 \mathrm{e}-5 \pm 5.7664 \mathrm{e}-7$ & $-1.3721 \mathrm{e}-5 \pm 6.0939 \mathrm{e}-7$ \\
2 & $-1.2886 \mathrm{e}-5 \pm 4.4730 \mathrm{e}-7$ & $-1.4850 \mathrm{e}-5 \pm 5.4374 \mathrm{e}-7$ \\
3 & $-1.3830 \mathrm{e}-5 \pm 4.2069 \mathrm{e}-7$ & $-1.5498 \mathrm{e}-5 \pm 4.9182 \mathrm{e}-7$ \\
4 & $-1.5150 \mathrm{e}-5 \pm 4.0328 \mathrm{e}-7$ & $-1.7550 \mathrm{e}-5 \pm 4.7749 \mathrm{e}-7$ \\
5 & $-1.6663 \mathrm{e}-5 \pm 3.9826 \mathrm{e}-7$ & $-1.9936 \mathrm{e}-5 \pm 5.0037 \mathrm{e}-7$ \\
6 & $-2.0076 \mathrm{e}-5 \pm 4.5347 \mathrm{e}-7$ & $-2.2165 \mathrm{e}-5 \pm 5.2553 \mathrm{e}-7$ \\
7 & $-2.1264 \mathrm{e}-5 \pm 4.5479 \mathrm{e}-7$ & $-2.4113 \mathrm{e}-5 \pm 5.5385 \mathrm{e}-7$ \\
8 & $-2.4969 \mathrm{e}-5 \pm 7.9908 \mathrm{e}-7$ & $-2.7581 \mathrm{e}-5 \pm 9.5344 \mathrm{e}-7$ \\
\hline
\end{tabular}

Table 5.17: The tracking efficiency vs chamber intensity is fitted with $y=\exp \left(p_{0} x\right)$. The value of the parameter $p_{0}$ and its error for each target and for each $x_{2}$ are listed. 

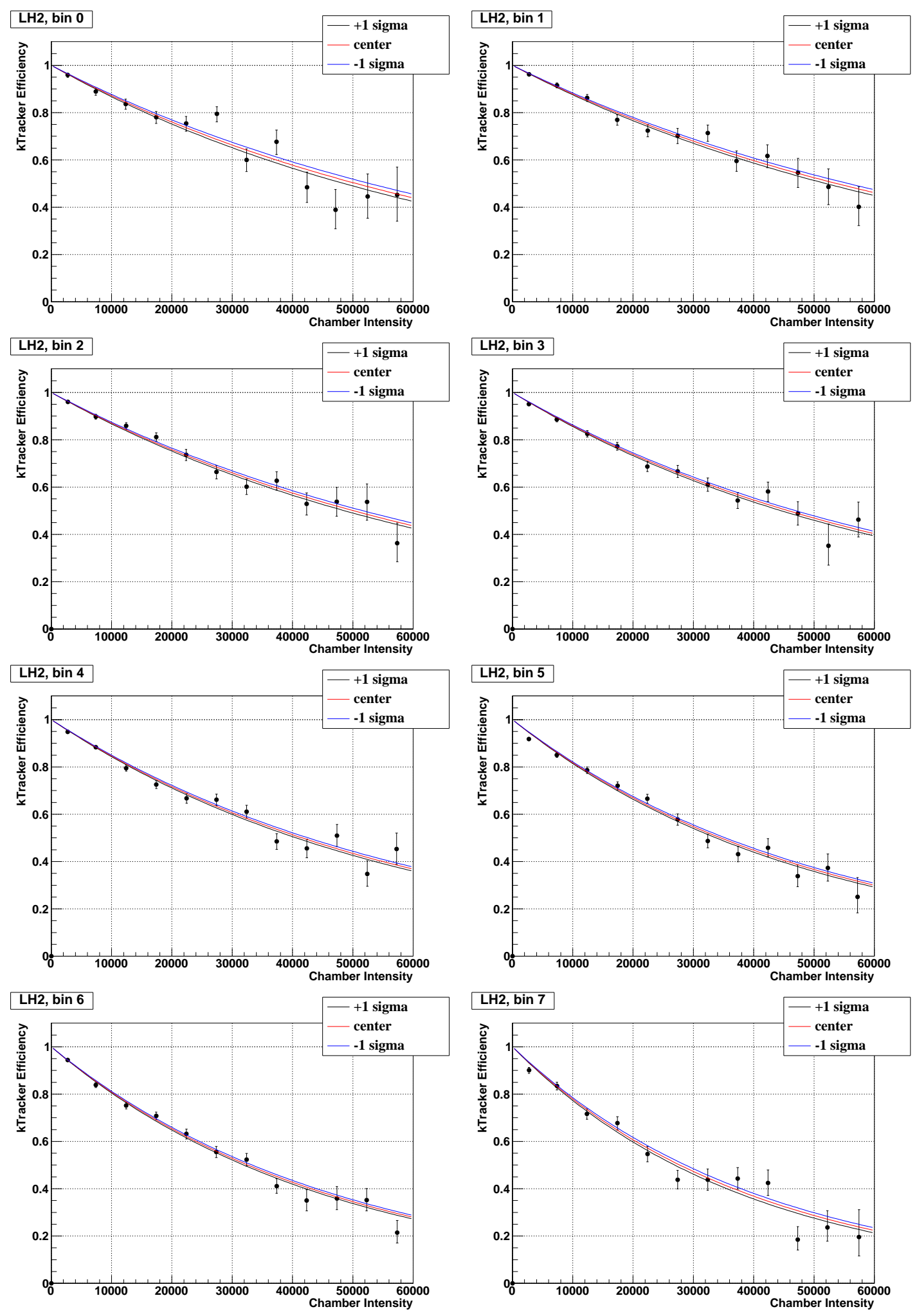

Figure 5.29: Tracking efficiency as a function of chamber intensity for hydrogen target for each $x_{2}$ bin. They are fitted to the exponential curve, $\exp \left(p_{0} I\right)$ (red curve). Blue and black curves correspond to $p_{0}$ shifted by $+1 \sigma$ or $-1 \sigma$ from the original (red) value. These are obtained using the MC simulations. 

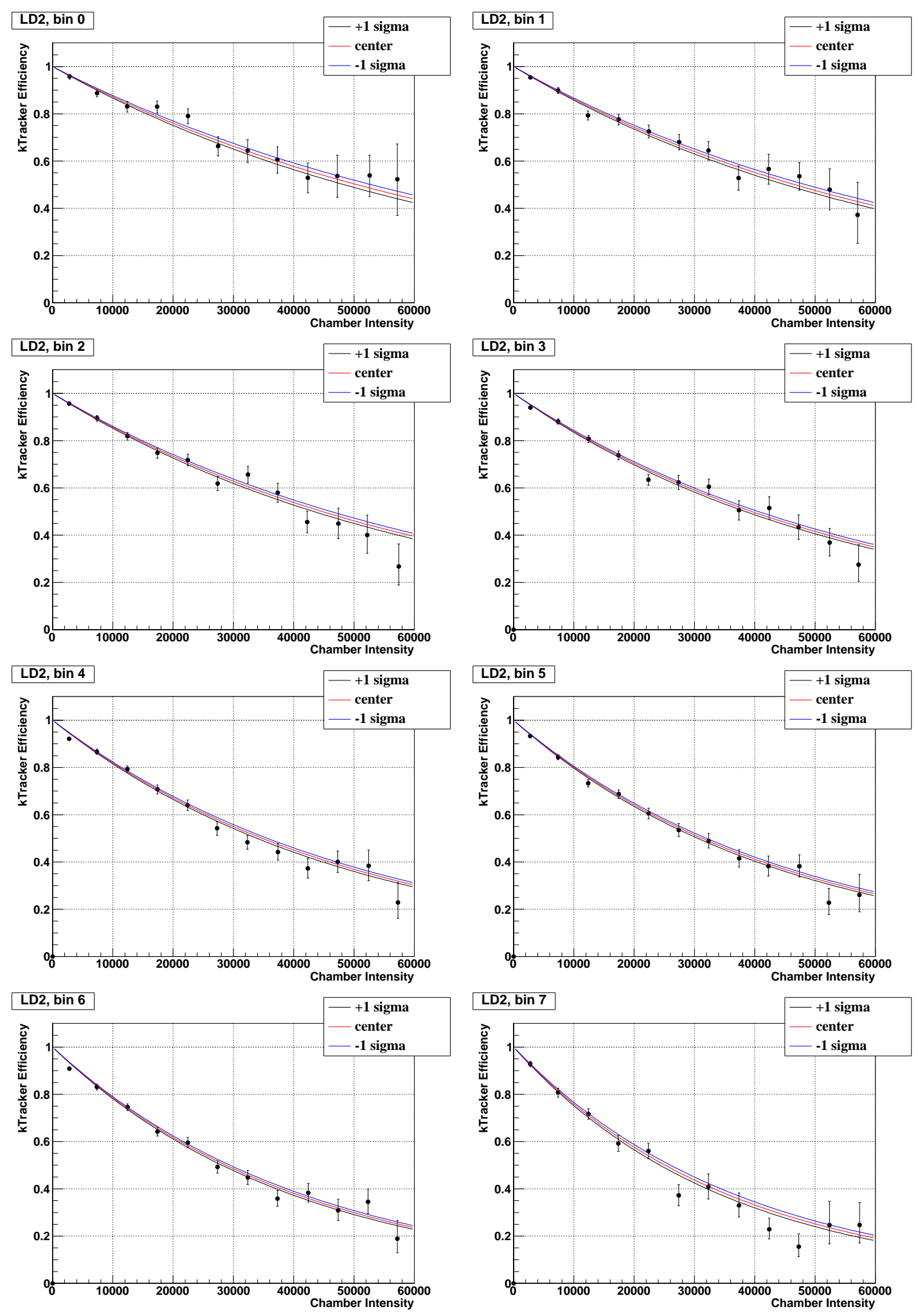

Figure 5.30: Tracking efficiency as a function of chamber intensity for deuterium target for each $x_{2}$ bin. They are fitted to the exponential curve, $\exp \left(p_{0} I\right)$ (red curve). Blue and black curves correspond to $p_{0}$ shifted by $+1 \sigma$ or $-1 \sigma$ from the original (red) value. These are obtained using the MC simulations. 

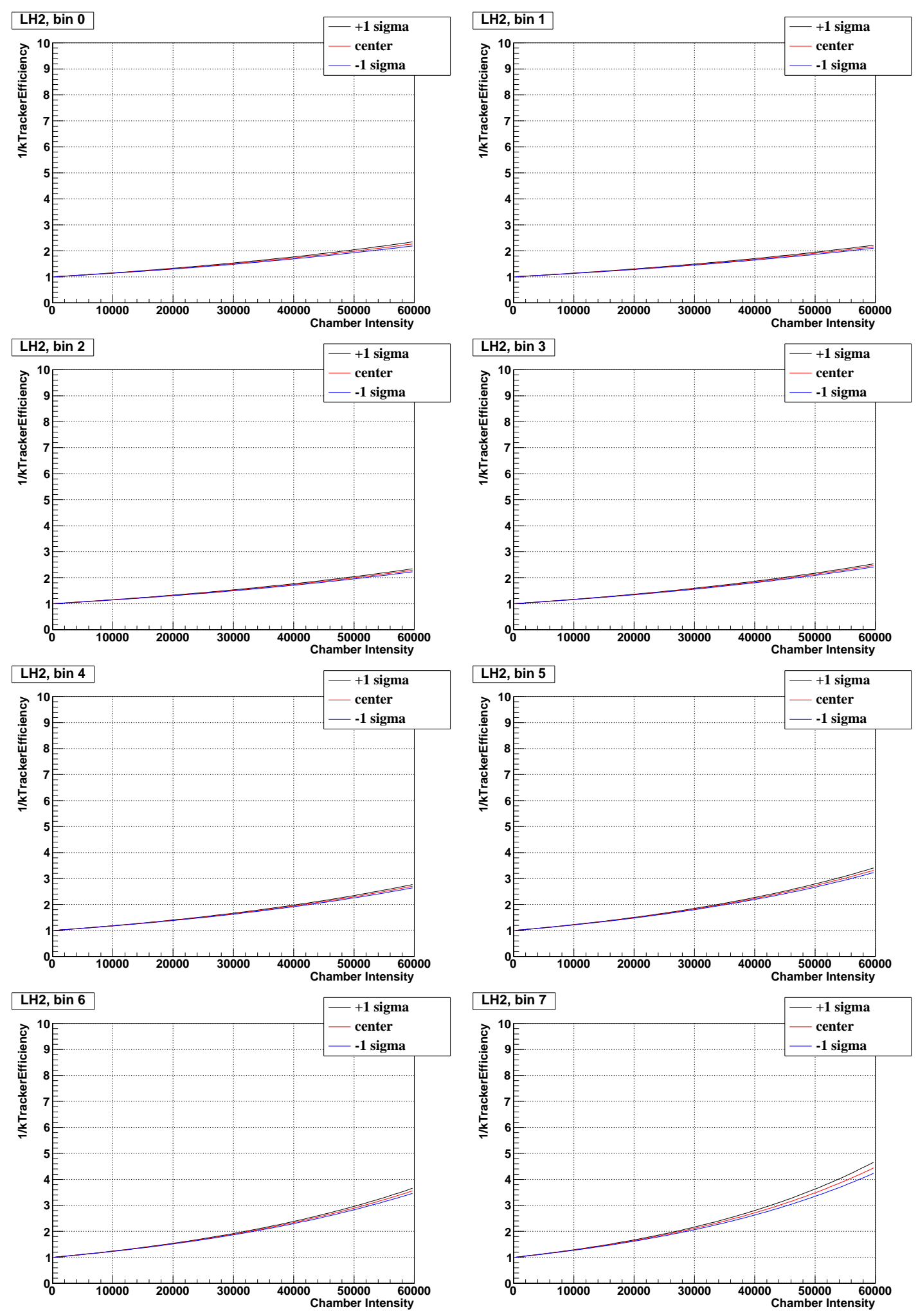

Figure 5.31: Weight, $1 / \exp \left(p_{0} I\right)$, as a function of chamber intensity for hydrogen target is shown in red for each $x_{2}$ bin. Blue and black curves correspond to $p_{0}$ shifted by $+1 \sigma$ or $-1 \sigma$ from the original (red) value. These are obtained using the $\mathrm{MC}$ simulations. 

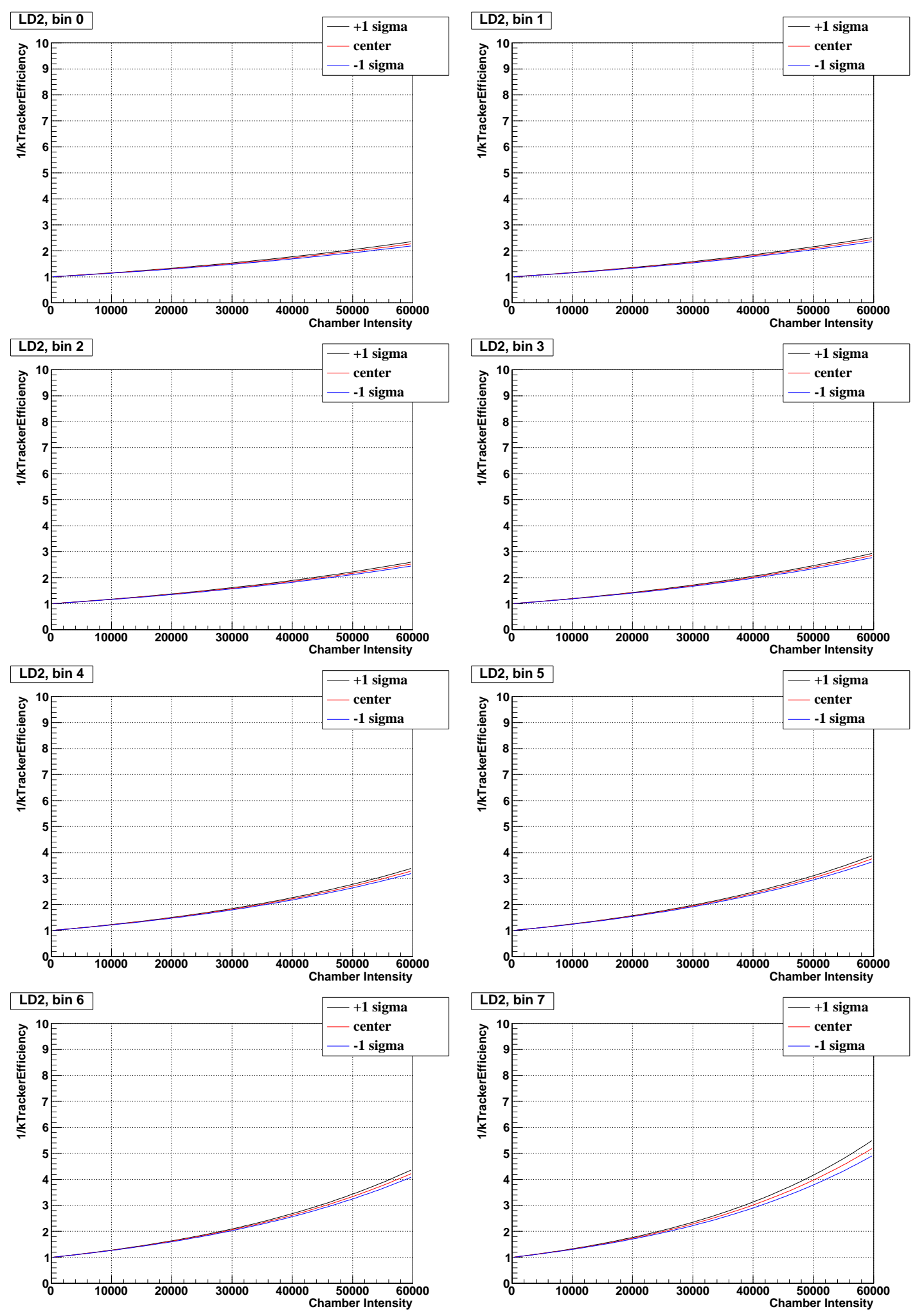

Figure 5.32: Weight, $1 / \exp \left(p_{0} I\right)$, as a function of chamber intensity for deuterium target is shown in red for each $x_{2}$ bin. Blue and black curves correspond to $p_{0}$ shifted by $+1 \sigma$ or $-1 \sigma$ from the original (red) value. These are obtained using the MC simulations. 


\subsubsection{Intensity dependence of combinatorial background}

Events are divided by its chamber intensity bin in order to know the behavior of combinatorial background as a function of chamber intensity. Mass distributions are made for each intensity. The fitting analysis like Fig. 5.15 was then done for each distribution. Figure 5.3 .3 and 5.34 show the results.

Ratio of amount of the combinatorial background per Drell-Yan events at high mass region $\left(\mathrm{M}>4.2 \mathrm{GeV} / \mathrm{c}^{2}\right)$ is calculated for each mass distribution. The ratio as a function of chamber intensity was then plotted (see Fig. 5.3.5). The fraction of the combinatorial background linearly increases as the intensity increases. It goes to zero in the limit when the intensity goes zero. The slope of LH2 target and that of LD2 target are the same. This fact means that the combinatorial background doesn't need to be corrected when calculating the cross section ratio.

\subsubsection{Cut on Chamber Intensity}

As can be seen in Fig. 5.31 and Fig. 5.32, the weight value becomes larger at higher chamber intensity, and its error size also becomes larger. It is decided that the events with the chamber intensity larger than 40000 are not used in the analysis so that the weight doesn't exceed 3.0. The systematic error at chamber intensity $=40000$ is $\sim 4 \%$, which is larger than the other sources of systematic errors (the systematic errors will be discussed later in this thesis). Also, this chamber intensity cut helps to suppress the contribution of the combinatorial background. The fraction of the combinatorial background to Drell-Yan events is 0.3 at chamber intensity $=40000$ as can be seen in Fig. 5.35. Based on these considerations, the cut is applied at 40000 . Roughly $80 \%$ of events remain after this cut. 
TargetPos: 1, Bin 1

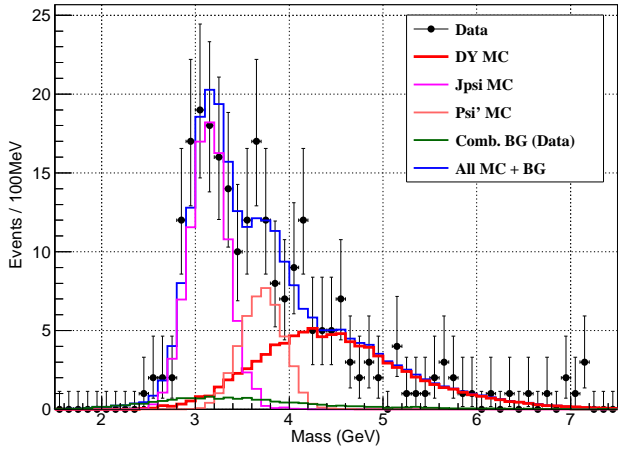

TargetPos: 1, Bin 3

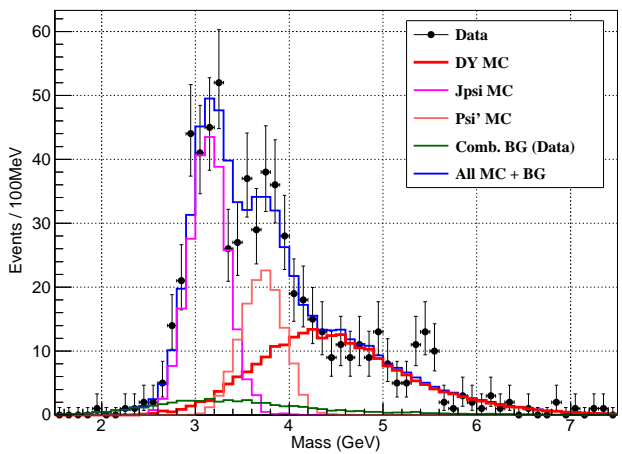

TargetPos: 1, Bin 5

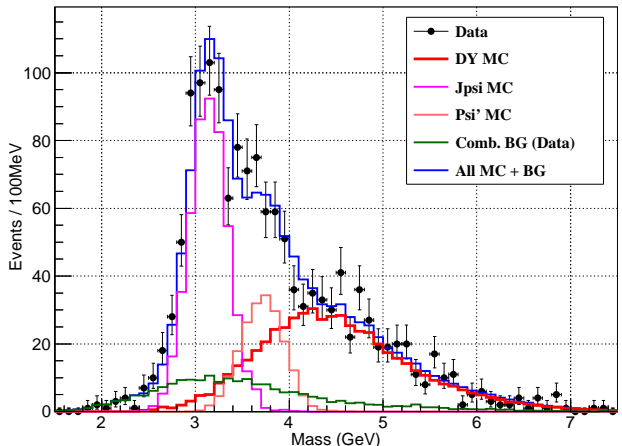

TargetPos: 1, Bin 7

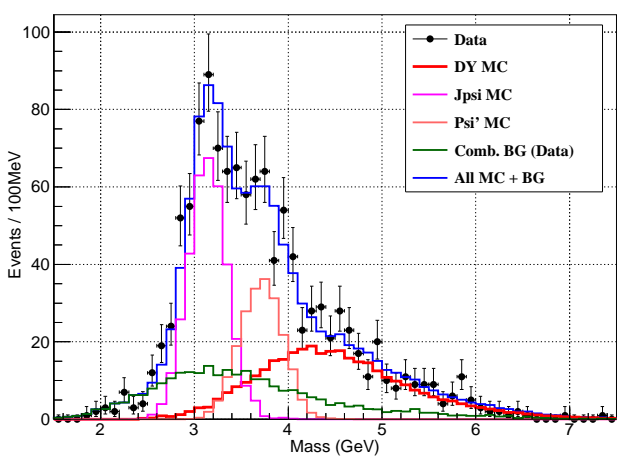

TargetPos: 1 , Bin 2

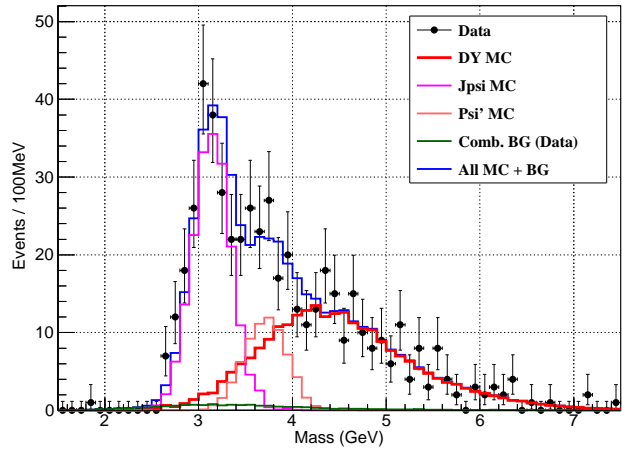

TargetPos: 1, Bin 4

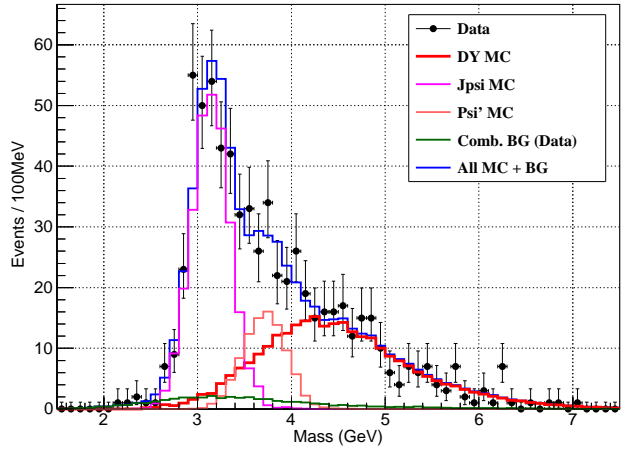

TargetPos: 1, Bin 6

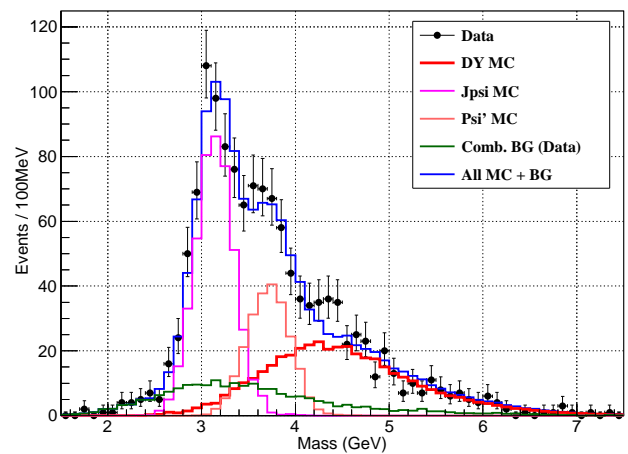

TargetPos: 1, Bin 8

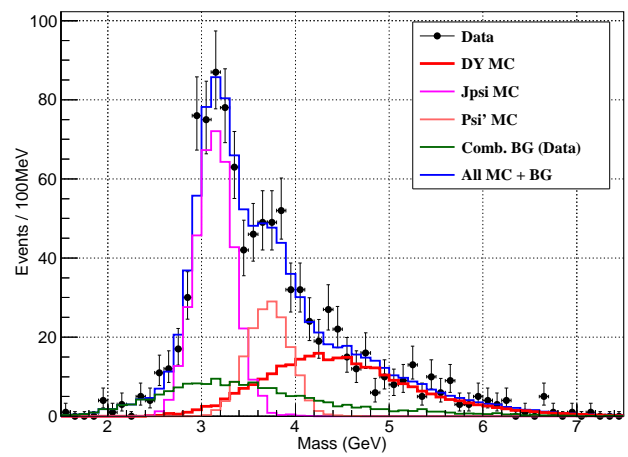

Figure 5.33: Fitting results of mass distribution from the 1st to 8th intensity bin for LH2 target. 

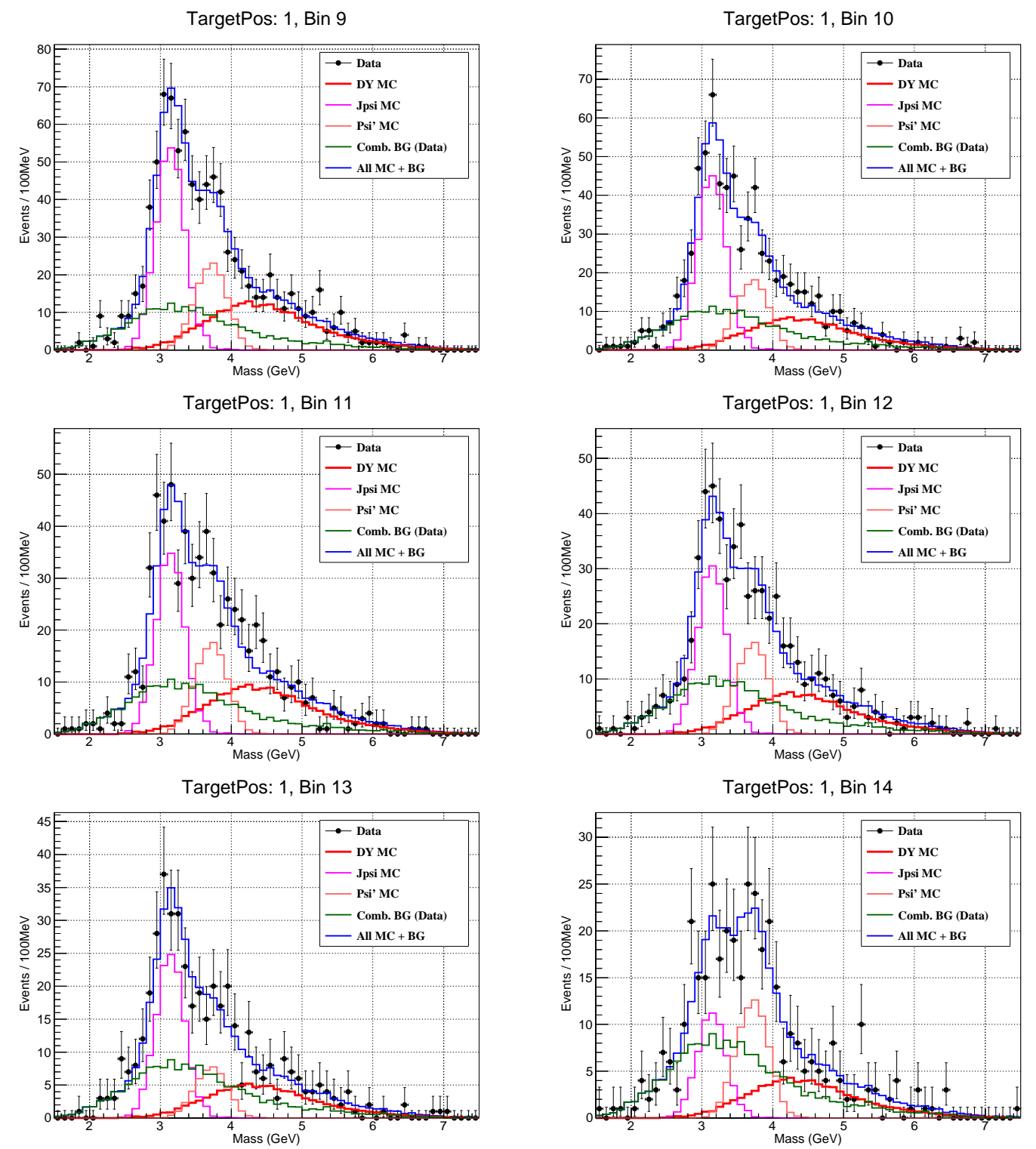

Figure 5.34: Fitting results of mass distribution from the 9th to 14th intensity bin for LH2 target. 

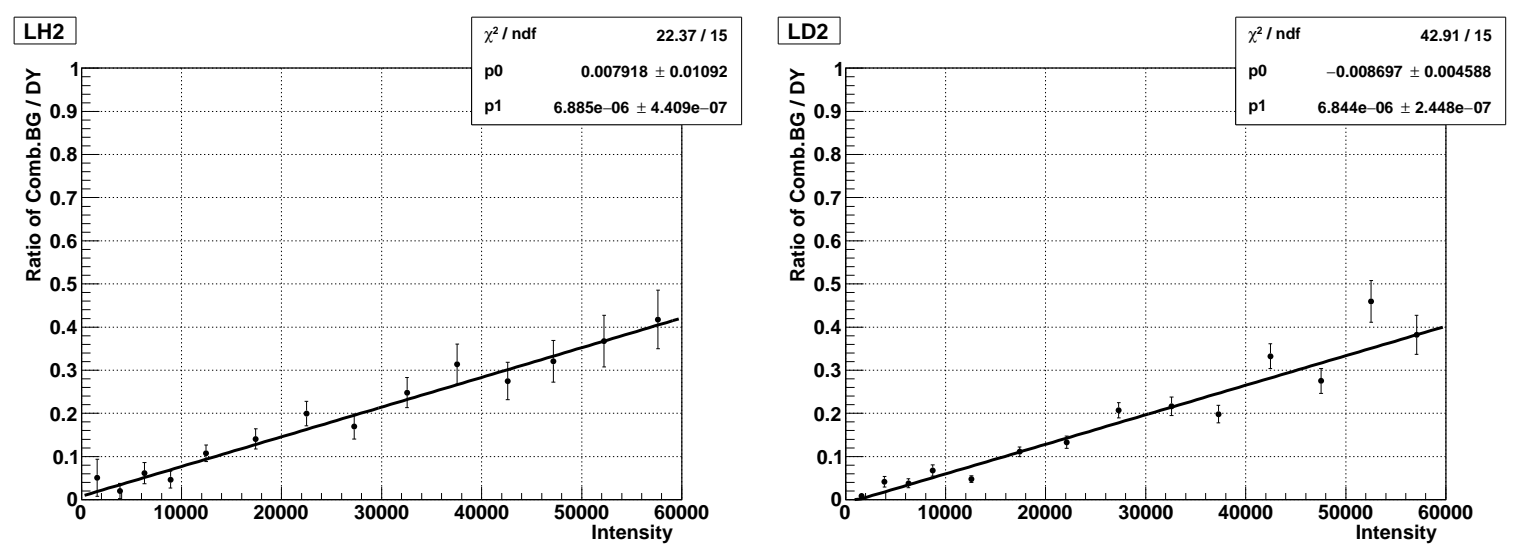

Figure 5.35: The fraction of amount of the combinatorial background per Drell-Yan events as a function of chamber intensity for LH2 target event (left) and LD2 target event (right). The fraction of the combinatorial background linearly increases as the intensity increases, and it goes to zero in the limit where intensity goes to zero. 


\subsubsection{Target contamination correction}

The next step is to know the exact number of hydrogen and deuterium in the target. The SeaQuest LD2 target was emptied and filled several times while we were taking data. At each time, a sample of the deuterium gas was taken from the target flask as it was warmed up or from the deuterium gas cylinder that was used to fill the target flask. These samples were analyzed and the results are shown in Table 5.18. The third sample has a significant fraction of $\mathrm{N}_{2}$ and $\mathrm{O}_{2}$, this indicated a contamination of air.

\begin{tabular}{l|rrr}
\hline \hline Components & Run 3230-11143 & Run $11144-11477$ & Run 11478-14652 \\
\hline $\mathrm{H}_{2}$ & $1.230 \%$ & $0.250 \%$ & $4.603 \%$ \\
$\mathrm{HD}$ & $16.187 \%$ & $8.393 \%$ & $6.983 \%$ \\
$\mathrm{D}_{2}$ & $79.780 \%$ & $90.871 \%$ & $80.504 \%$ \\
$\mathrm{He}$ & $2.200 \%$ & $0.018 \%$ & $<0.01 \%$ \\
$\mathrm{~N}_{2}$ & $0.428 \%$ & $0.323 \%$ & $6.395 \%$ \\
$\mathrm{O}_{2}$ & $0.085 \%$ & $0.068 \%$ & $1.389 \%$ \\
$\mathrm{Ar}$ & $0.005 \%$ & $0.004 \%$ & $0.063 \%$ \\
\hline
\end{tabular}

Table 5.18: The result of gas analysis expressed in mole percent for the LD2 target for each run period.

All samples were taken when the deuterium was in a gaseous state. So the analyses are not perfect to know what we have in the target when the deuterium was in a liquid state. As the deuterium was cooled, the heavier components $\left(\mathrm{N}_{2}, \mathrm{O}_{2}\right.$, Ar) should have been frozen out of the liquid. Also, Helium existed in a gaseous state top of the target flask, and didn't interact with the proton beam. Thus, the gases shown in Table 5.18 can be renormalized to have only three components: $\mathrm{H}_{2}, \mathrm{D}_{2}$ and HD. Also, the values are averaged over Roadset period. This is shown in Table 5.19. Since the values of gas analysis are not the values during data taking, SeaQuest for the moment decided to put extreme systematic uncertainty on the fraction of $\mathrm{D}_{2}$, which is $\pm 5 \%$. On the other hand, the LH2 target we used during the data taking was 100\% pure.

Constants of target materials used in the analysis are listed in Table. 5201 [37]. The number density, $n$, and nuclear interaction length, $\lambda$, can be corrected for as follows using the target fraction, $C$. The superscript of $C$ and $\lambda$ denotes the target type, and the subscript of those denotes components in it. 


\begin{tabular}{rl|rr}
\hline \hline Roadset & Components & LH2 target & LD2 target \\
\hline \multirow{3}{*}{57} & $\mathrm{H}_{2}$ & $100.00 \%$ & $1.26 \%$ \\
& $\mathrm{D}_{2}$ & $0.00 \%$ & $82.08 \%$ \\
& $\mathrm{HD}$ & $0.00 \%$ & $16.65 \%$ \\
\hline \multirow{3}{*}{62} & $\mathrm{H}_{2}$ & $100.00 \%$ & $4.90 \%$ \\
& $\mathrm{D}_{2}$ & $0.00 \%$ & $87.49 \%$ \\
& $\mathrm{HD}$ & $0.00 \%$ & $7.61 \%$ \\
\hline \multirow{3}{*}{67} & $\mathrm{H}_{2}$ & $100.00 \%$ & $2.70 \%$ \\
& $\mathrm{D}_{2}$ & $0.00 \%$ & $93.20 \%$ \\
& $\mathrm{HD}$ & $0.00 \%$ & $4.10 \%$ \\
\hline
\end{tabular}

Table 5.19: The fractions in LH2 target and LD2 target are listed for Roadset 57, 62 and 67 in percentage. The LH2 target is $100 \%$ pure.

\begin{tabular}{llll}
\hline \hline Target & Constant & & Value \\
\hline $\mathrm{H}_{2}$ & Nuclear interaction length & $\lambda_{H 2}$ & $734.6 \mathrm{~cm}$ \\
& Density & $\rho_{H 2}$ & $0.0708 \mathrm{~g} / \mathrm{cm}^{3}$ \\
& Atomic mass & $m_{H 2}^{a t m}$ & $1.00794 \mathrm{~g} / \mathrm{mol}^{3}$ \\
& Number density & $n_{H 2}$ & $0.0351 \mathrm{~mol} / \mathrm{cm}^{3}$ \\
\hline $\mathrm{D}_{2}$ & Nuclear interaction length & $\lambda_{D 2}$ & $424.7 \mathrm{~cm}$ \\
& Density & $\rho_{D 2}$ & $0.169 \mathrm{~g} / \mathrm{cm}^{3}$ \\
& Atomic mass & $m_{D 2}^{a t m}$ & $2.01410 \mathrm{~g} / \mathrm{mol}^{3}$ \\
& Number density & $n_{D 2}$ & $0.0420 \mathrm{~mol} / \mathrm{cm}^{3}$ \\
\hline
\end{tabular}

Table 5.20: Values of target materials [37].

\section{For LH2 target}

$$
\begin{aligned}
n_{p}^{L H 2} & =2 \cdot C_{H 2}^{L H 2} n_{H 2}+1 \cdot C_{H D}^{L H 2} n_{H D} \\
n_{d}^{L H 2} & =2 \cdot C_{D 2}^{L H 2} n_{D 2}+1 \cdot C_{H D}^{L H 2} n_{H D} \\
\lambda_{L H 2} & =1 /\left(C_{H 2}^{L H 2} / \lambda_{H 2}+C_{D 2}^{L H 2} / \lambda_{D 2}+C_{H D}^{L H 2} / \lambda_{H D}\right)
\end{aligned}
$$

\section{For LD2 target}

$$
\begin{aligned}
n_{p}^{L D 2} & =2 \cdot C_{H 2}^{L D 2} n_{H 2}+1 \cdot C_{H D}^{L D 2} n_{H D} \\
n_{d}^{L D 2} & =2 \cdot C_{D 2}^{L D 2} n_{D 2}+1 \cdot C_{H D}^{L D 2} n_{H D} \\
\lambda_{L D 2} & =1 /\left(C_{H 2}^{L D 2} / \lambda_{H 2}+C_{D 2}^{L D 2} / \lambda_{D 2}+C_{H D}^{L D 2} / \lambda_{H D}\right),
\end{aligned}
$$


where

$$
\begin{aligned}
n_{H D} & =\left(n_{H 2}+n_{D 2}\right) / 2 \\
\lambda_{H D} & =1 /\left(1 / \lambda_{L H 2}+1 / \lambda_{L D 2}\right)
\end{aligned}
$$

\subsubsection{Formula of cross section ratio}

Raw number of di-muon events from LH2 or LD2 target are expressed as below.

$$
\begin{aligned}
& D_{L H 2}-B_{L H 2}=P_{L H 2} \cdot n_{p}^{L H 2} \cdot l_{L H 2} \cdot \sigma^{p p}+P_{L H 2} \cdot n_{d}^{L H 2} \cdot l_{L H 2} \cdot \sigma^{p d} \\
& D_{L D 2}-B_{L D 2}=P_{L D 2} \cdot n_{p}^{L D 2} \cdot l_{L D 2} \cdot \sigma^{p p}+P_{L D 2} \cdot n_{d}^{L D 2} \cdot l_{L D 2} \cdot \sigma^{p d},
\end{aligned}
$$

where $D$ is dimuon yield from target, $B$ background for the target, $P$ number of live beam proton, $\sigma^{p p}$ and $\sigma^{p d}$ Drell-Yan cross section of $p-p$ or $p$ - $d$ interaction, respectively, and $l$ is an effective target length. The subscripts denote target type. The background, $B$, is obtained from empty target events as:

$$
\begin{aligned}
& B_{L H 2}=\frac{P_{L H 2}}{P_{E}} \cdot D_{E} \\
& B_{L D 2}=\frac{P_{L D 2}}{P_{E}} \cdot D_{E},
\end{aligned}
$$

where the subscript, $E$, denotes the empty event. The effective target length, $l$, are calculated as:

$$
\begin{aligned}
& l_{L H 2}=\lambda_{L H 2}\left(1-\exp \left(-L / \lambda_{L H 2}\right)\right) \\
& l_{L D 2}=\lambda_{L D 2}\left(1-\exp \left(-L / \lambda_{L D 2}\right)\right),
\end{aligned}
$$

where $L$ is the target flask length, which is $50.8 \mathrm{~cm}$. From Eq. 5.22, 5.231, formula of cross section ratio is derived as

$$
\frac{\sigma^{p d}}{\sigma^{p p}}=\frac{A n_{p}^{L H 2}-n_{p}^{L D 2}}{n_{d}^{L D 2}-A n_{d}^{L H 2}}
$$

where

$$
A=\frac{P_{L H 2} l_{L H 2}\left(D_{L D 2}-B_{L D 2}\right)}{P_{L D 2} l_{L D 2}\left(D_{L H 2}-B_{L H 2}\right)}
$$

Now, since $n_{d}^{L H 2}$ is negligible, Eq. 5.28 can be reduced to

$$
\frac{\sigma^{p d}}{\sigma^{p p}}=\frac{P_{L H 2} l_{L H 2} n_{p}^{L H 2}\left(D_{L D 2}-B_{L D 2}\right)}{P_{L D 2} l_{L D 2} n_{d}^{L D 2}\left(D_{L H 2}-B_{L H 2}\right)}-\frac{n_{p}^{L D 2}}{n_{d}^{L D 2}} .
$$




\subsection{Systematic error}

Systematic error on the cross section ratio is calculated in this section. Table 5.27 is the list of the sources of the systematic errors. Each of sources is described in next subsections.

\begin{tabular}{ll}
\hline \hline Source of error & Error $(\%)$ \\
\hline Correction of intensity dependence & $\sim 0.1 \%$ \\
Intensity dependence on cross section ratio & $0.9 \%$ \\
Correction of target contamination & $\sim 1.5 \%$ \\
Target length & $0.2 \%$ \\
Bjorken $x_{2}$ resolution & $3.3 \%$ \\
Beam intensity -absolute value & negligible \\
Beam intensity -pedestal & negligible \\
Beam intensity -saturation & negligible \\
\hline
\end{tabular}

Table 5.21: Sources of systematic errors.

\subsubsection{Correction of intensity dependence}

The intensity dependence is corrected as described in Sec. 5.9.3. Each dimuon event is weighted by $1 / \exp \left(p_{0} I\right)$, and $p_{0}$ has a systematic error. Now the effect of the systematic error on the cross section ratio is considered. The cross section ratio is calculated three times: one with nominal factors $\left(p_{0}\right)$, one with factors increased by one sigma $\left(p_{0}+\sigma\right)$ and one with factors decreased by one sigma $\left(p_{0}-\sigma\right)$. The obtained cross section ratios are named as $R_{\text {base }}, R_{\text {plus }}$, and $R_{\text {minus }}$. Then the upper systematic error, $\delta^{+}$, and lower systematic error, $\delta^{-}$, are obtained for each $x_{2}$ bin by:

$$
\begin{aligned}
& \delta_{\text {int }}^{-}=\left|R_{\text {base }}-R_{\text {minus }}\right| \\
& \delta_{\text {int }}^{+}=\left|R_{\text {base }}-R_{\text {plus }}\right|
\end{aligned}
$$

Table 5.10.] summarize the systematic errors $\left(\delta_{\text {int }}^{-}\right.$and $\left.\delta_{\text {int }}^{+}\right)$due to the intensity dependence correction for each roadset data and for each $x_{2}$ bin. 


\begin{tabular}{l|l|llll}
\hline \hline Roadset & Bin & \multicolumn{3}{|c}{$\delta_{\text {int }}^{-}$} & \multicolumn{2}{c}{$\delta_{\text {int }}^{+}$} \\
\hline \multirow{5}{*}{57} & 1 & 0.00206 & $0.167 \%$ & 0.00210 & $0.170 \%$ \\
& 2 & 0.00001 & $0.002 \%$ & $<0.00001$ & $<0.001 \%$ \\
& 3 & 0.00128 & $0.118 \%$ & 0.00130 & $0.120 \%$ \\
& 4 & 0.00059 & $0.052 \%$ & 0.00060 & $0.053 \%$ \\
& 5 & 0.00903 & $0.548 \%$ & 0.00933 & $0.566 \%$ \\
& 6 & 0.00029 & $0.028 \%$ & 0.00028 & $0.027 \%$ \\
& 7 & 0.05297 & $1.356 \%$ & 0.05541 & $1.418 \%$ \\
& 8 & 0.01389 & $0.494 \%$ & 0.01369 & $0.487 \%$ \\
\hline \multirow{5}{*}{62} & 1 & 0.00023 & $0.022 \%$ & 0.00025 & $0.024 \%$ \\
& 2 & 0.00065 & $0.053 \%$ & 0.00067 & $0.055 \%$ \\
& 3 & 0.00105 & $0.088 \%$ & 0.00108 & $0.090 \%$ \\
& 4 & 0.00116 & $0.105 \%$ & 0.00119 & $0.108 \%$ \\
& 5 & 0.00031 & $0.036 \%$ & 0.00032 & $0.036 \%$ \\
& 6 & 0.00015 & $0.013 \%$ & 0.00016 & $0.014 \%$ \\
& 7 & 0.00221 & $0.151 \%$ & 0.00226 & $0.155 \%$ \\
& 8 & 0.00644 & $0.566 \%$ & 0.00638 & $0.561 \%$ \\
\hline \multirow{5}{*}{67} & 1 & 0.00109 & $0.080 \%$ & 0.00111 & $0.081 \%$ \\
& 2 & 0.00049 & $0.039 \%$ & 0.00051 & $0.040 \%$ \\
& 3 & 0.00121 & $0.092 \%$ & 0.00124 & $0.095 \%$ \\
& 4 & 0.00060 & $0.050 \%$ & 0.00062 & $0.051 \%$ \\
& 5 & 0.00175 & $0.125 \%$ & 0.00179 & $0.128 \%$ \\
& 6 & 0.00137 & $0.115 \%$ & 0.00138 & $0.116 \%$ \\
& 7 & 0.00224 & $0.162 \%$ & 0.00228 & $0.165 \%$ \\
& 8 & 0.00056 & $0.073 \%$ & 0.00056 & $0.073 \%$ \\
\hline \multirow{6}{*}{} & & & & & \\
& & & & \\
& & & \\
& & &
\end{tabular}

Table 5.22: Systematic errors due to the intensity dependence correction for each Roadset data and for each $x_{2}$ bin. 


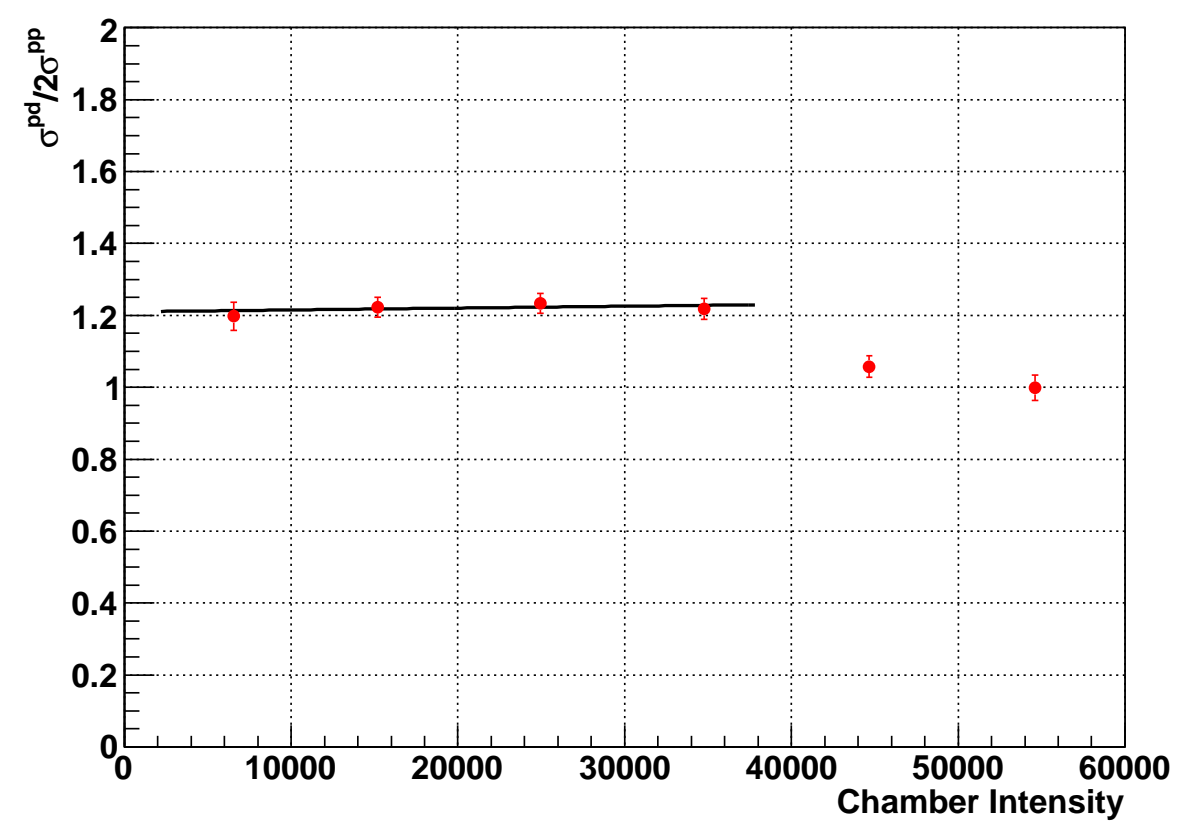

Figure 5.36: Cross section ratio as a function of chamber intensity after the intensity dependence correction was applied. As described in Sec. 5.9.5, the data at 0-40000 are used for the analysis. It can be seen in this figure that the cross section ratio is mostly flat at $0-40000$.

\subsubsection{Intensity dependence on cross section ratio}

Now, the effect of the intensity dependence correction on the cross section ratio is considered. Figure 5.36 shows the cross section ratio as a function of chamber intensity after the intensity dependence correction was applied. As described in Sec. 5.9.5, the data at 0-40000 are used for the analysis. The cross section ratio is almost flat at 0-40000. The cross section ratio is shown with the data of 0-10000, 0-20000, and so on in order to check the effect of the cut on the chamber intensity in Fig. 5.37. It can be seen that the cross section ratio is stable. The remaining dependence is treated as a systematic error. The size of the systematic error is $0.9 \%$. Further analysis will be done in future in order to handle the remaining dependence. 


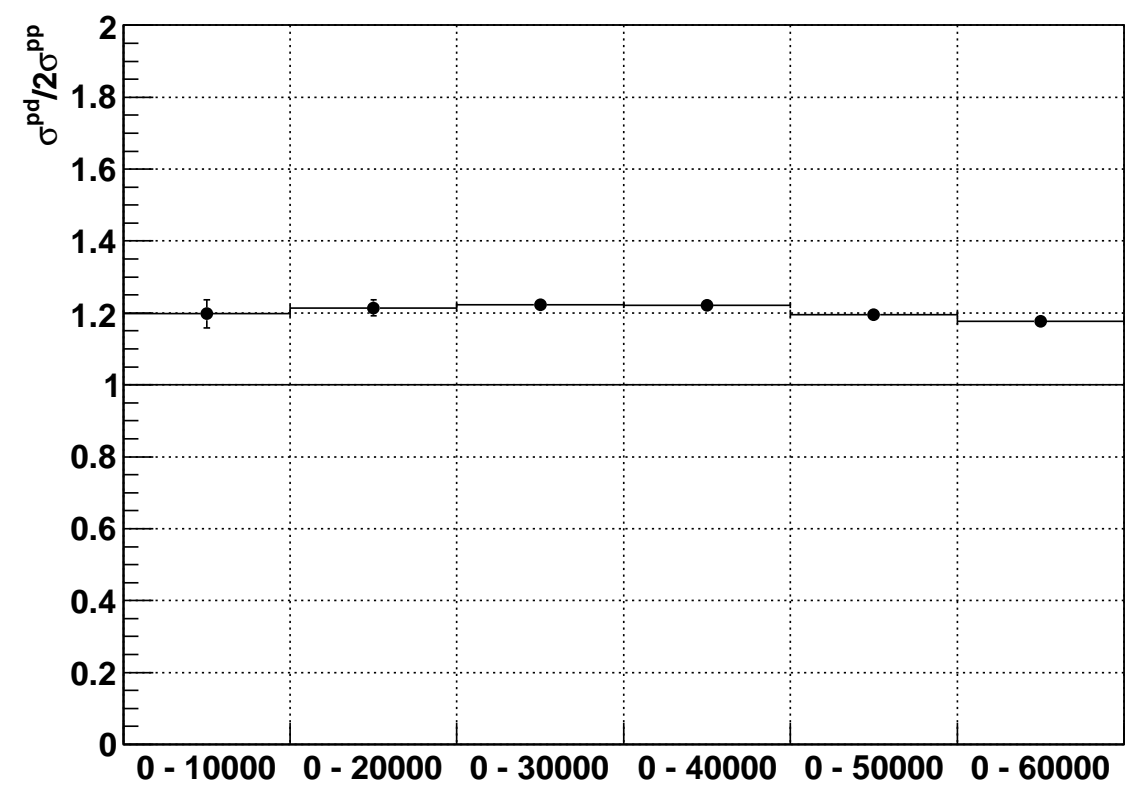

Figure 5.37: The cross section ratio with the data of 0-10000, 0-20000, and so on in order to check the effect of the cut on the chamber intensity. It can be seen that the cross section ratio is stable. 


\subsubsection{Correction of target contamination}

As mentioned in Sec. 5.9.6, SeaQuest decided to put extreme systematic error on the fraction of $\mathrm{D}_{2}$ in LD2 target since the target contamination analysis is ongoing. The magnitude of the error is $\pm 5 \%$. This value can be reduced in future.

Th effect of the systematic error on the cross section ratio is calculated in the same way as we did for the intensity dependence correction in Sec. 5.10.7. The cross section ratio is calculated three times; $R_{\text {base }}, R_{\text {plus }}$, and $R_{\text {minus }}$. Then the upper systematic error, $\delta^{+}$, and lower systematic error, $\delta^{-}$, are obtained for each $x_{2}$ bin by:

$$
\begin{aligned}
& \delta_{\text {targ }}^{-}=\left|R_{\text {base }}-R_{\text {minus }}\right| \\
& \delta_{\text {targ }}^{+}=\left|R_{\text {base }}-R_{\text {plus }}\right|
\end{aligned}
$$

Table 5.10 .3 summarize the systematic errors due to the target contamination correction $\left(\delta_{\text {targ }}^{-}\right.$and $\left.\delta_{\text {targ }}^{+}\right)$for each Roadset data and for each $x_{2}$ bin.

\subsubsection{Target length}

The systematic error from the target length is due to a known slight difference between the length of the target flask of LH2 target and LD2 target. It is unknown which flask is longer. The magnitude of the systematic error on the cross section ratio by the target length is $0.2 \%$ [42].

\subsubsection{Bjorken $x_{2}$ resolution}

The Bjorken $x_{2}$ resolution is roughly $10 \%$ and the value shifts by $\sim 0.01$ after track reconstruction [4:3]. The effect of the $x_{2}$ resolution on the cross section ratio is checked. Two cross section ratios as a function of $x_{2}$ are obtained using GMC as can be seen in Fig. 5.38. The decreasing ratio is simple due to the parton distributions adopted in the GMC. Red points show the cross section ratio as a function of reconstructed $x_{2}$. Black points show the ratio as a function of true $x_{2}$. A difference between these two cross section ratios is $\sim 3.3 \%$. For now, this value is included in systematic error. $x_{2}$ correction will be done in future analysis. 


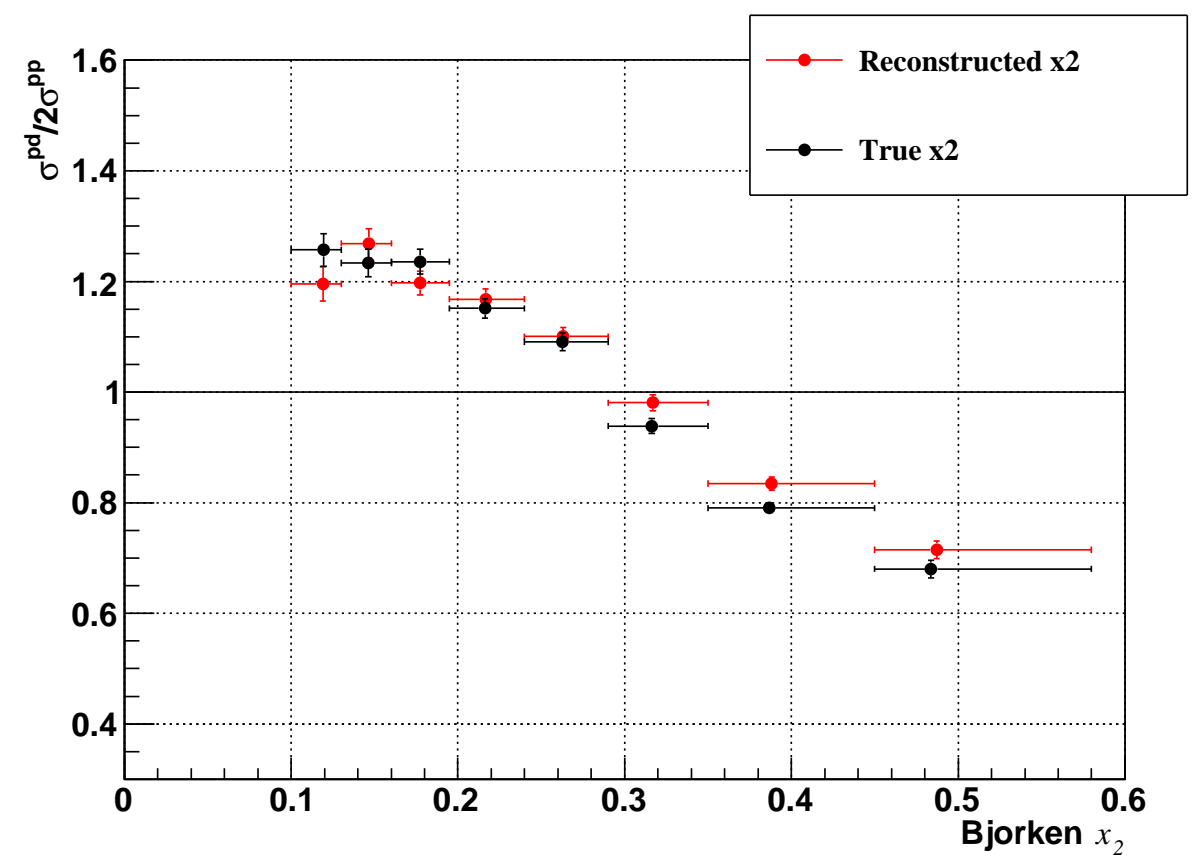

Figure 5.38: Cross section ratios as a function of $x_{2}$ obtained using GMC. Red points show the cross section ratio as a function of reconstructed $x_{2}$. Black points show the ratio as a function of true $x_{2}$. 


\begin{tabular}{l|l|llll}
\hline \hline Roadset & Bin & \multicolumn{2}{|c}{$\delta_{\text {targ }}^{-}$} & \multicolumn{2}{c}{$\delta_{\text {targ }}^{+}$} \\
\hline \multirow{5}{*}{57} & 1 & 0.02247 & $1.815 \%$ & 0.02381 & $1.924 \%$ \\
& 2 & 0.01894 & $1.704 \%$ & 0.02006 & $1.805 \%$ \\
& 3 & 0.01823 & $1.678 \%$ & 0.01932 & $1.779 \%$ \\
& 4 & 0.01949 & $1.723 \%$ & 0.02064 & $1.825 \%$ \\
& 5 & 0.03402 & $2.063 \%$ & 0.03604 & $2.186 \%$ \\
& 6 & 0.01701 & $1.632 \%$ & 0.01801 & $1.728 \%$ \\
& 7 & 0.09741 & $2.493 \%$ & 0.10319 & $2.641 \%$ \\
& 8 & 0.06668 & $2.371 \%$ & 0.07063 & $2.511 \%$ \\
\hline \multirow{5}{*}{62} & 1 & 0.01590 & $1.508 \%$ & 0.01676 & $1.589 \%$ \\
& 2 & 0.02027 & $1.656 \%$ & 0.02137 & $1.745 \%$ \\
& 3 & 0.01949 & $1.632 \%$ & 0.02055 & $1.721 \%$ \\
& 4 & 0.01721 & $1.557 \%$ & 0.01815 & $1.642 \%$ \\
& 5 & 0.01111 & $1.279 \%$ & 0.01172 & $1.349 \%$ \\
& 6 & 0.01768 & $1.573 \%$ & 0.01863 & $1.658 \%$ \\
& 7 & 0.02640 & $1.805 \%$ & 0.02783 & $1.903 \%$ \\
& 8 & 0.01802 & $1.585 \%$ & 0.01901 & $1.672 \%$ \\
\hline \multirow{5}{*}{67} & 1 & 0.01985 & $1.452 \%$ & 0.02541 & $1.859 \%$ \\
& 2 & 0.01784 & $1.402 \%$ & 0.02284 & $1.795 \%$ \\
& 3 & 0.01868 & $1.424 \%$ & 0.02390 & $1.822 \%$ \\
& 4 & 0.01645 & $1.362 \%$ & 0.02105 & $1.743 \%$ \\
& 5 & 0.02047 & $1.467 \%$ & 0.02620 & $1.877 \%$ \\
& 6 & 0.01617 & $1.354 \%$ & 0.02070 & $1.733 \%$ \\
& 7 & 0.02025 & $1.462 \%$ & 0.02593 & $1.871 \%$ \\
& 8 & 0.00702 & $0.917 \%$ & 0.00899 & $1.174 \%$ \\
\hline
\end{tabular}

Table 5.23: Systematic errors due to the target contamination correction for each Roadset data and for each $x_{2}$ bin. 


\title{
5.10.6 Beam intensity
}

Next, the accuracy of the beam intensity is checked. Three types of uncertainties on the beam intensity are checked.

- Absolute beam intensity

- Fluctuation of pedestal level

- Saturation of Cherenkov signal

\begin{abstract}
Absolute beam intensity
The absolute value of the beam intensity is measured by G2SEM as described in Sec. 4.2. The G2SEM reading has an error of 6.5\% [44]. But, since such an error is common to both LH2 and LD2 target events, the error on the cross section ratio is negligible. This was confirmed using a toy Monte Carlo simulation [44].
\end{abstract}

\section{Fluctuation of pedestal level}

The pedestal level can affect the RF bucket beam intensity (i.e. RF+00). As listed in Table 5.15, the fixed pedestal level is used for each spill range. But the pedestal level was not just constant. Figure 5.39 shows lower edge of the distribution of QIE value in one run [45]. It can be seen that the pedestal is fluctuated. The standard deviation of the pedestal is roughly 5 of QIE value [45].

The range of the QIE value of $\mathrm{RF}+00$ is typically from 500 to 2500 . Since the pedestal value deviates by \pm 5 , the pedestal-subtracted $R F+00$ varies by $\sim 1 \%(\sim 5 /(500-36.2))$. Its effect on the cross section ratio should be negligible.

\section{Saturation of Cherenkov signal}

Cherenkov-signal saturation was observed. The size of the saturation is roughly $3 \%$ at maximum [46]. The saturation happens only on high-intensity RF buckets (not all RF buckets equally). Such high-intensity events are rejected by QIE inhibit. Therefore, the saturation doesn't affect the RF bucket beam intensity of recorded events but just affects the normalization of beam intensity. Since the effect is common to both LH2 target and LD2 target events, the normalization error of $3 \%$ is negligible on cross section ratio. 


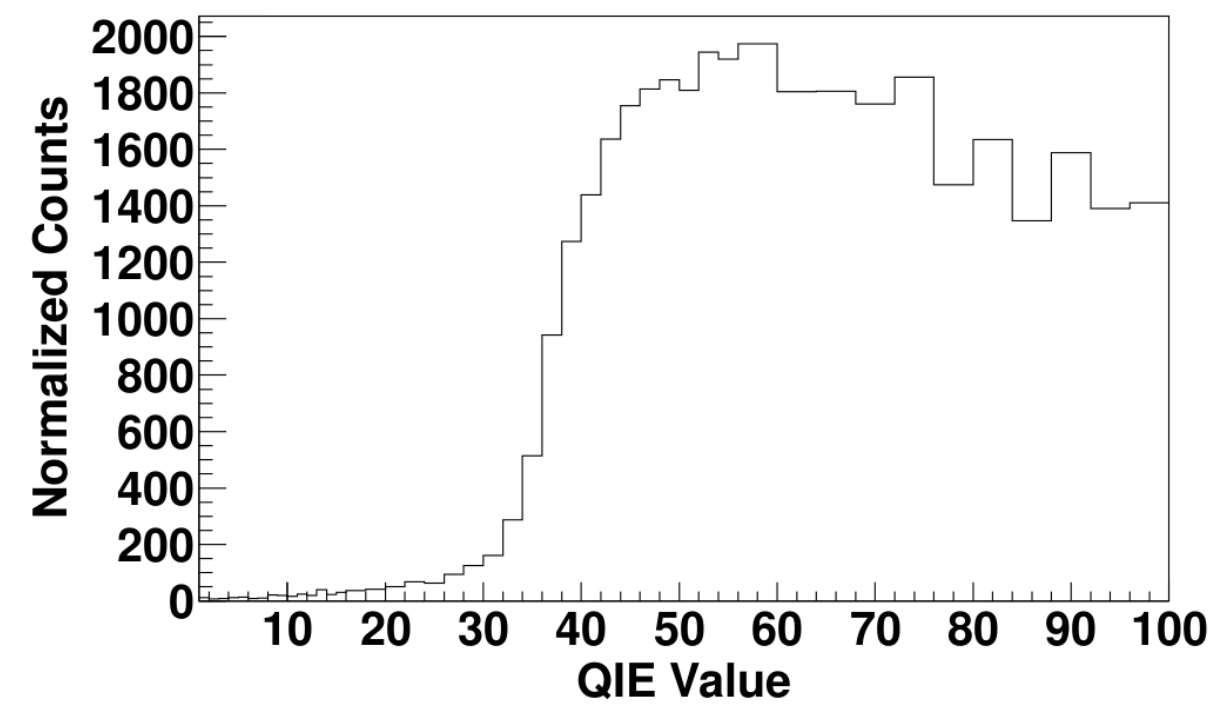

Figure 5.39: Lower edge of the distribution of QIE value in one run [4.5]. It can be seen that the pedestal is fluctuated.

\subsubsection{Total systematic error}

The total systematic error is obtained by this formula:

$$
\delta_{\mathrm{tot}}=\sqrt{\sum_{i} \delta_{i}^{2}}
$$

Upper errors and lower errors are calculated separately. Table 5.10.7 shows the total systematic errors for each Roadset data and for each $x_{2}$ bin. 


\begin{tabular}{l|l|llll}
\hline \hline Roadset & Bin & \multicolumn{2}{|c}{$\delta_{\text {tot }}^{-}$} & \multicolumn{2}{c}{$\delta_{\text {tot }}^{+}$} \\
\hline \multirow{5}{*}{57} & 1 & 0.04807 & $3.885 \%$ & 0.04871 & $3.936 \%$ \\
& 2 & 0.04257 & $3.830 \%$ & 0.04308 & $3.876 \%$ \\
& 3 & 0.04150 & $3.820 \%$ & 0.04199 & $3.866 \%$ \\
& 4 & 0.04342 & $3.839 \%$ & 0.04394 & $3.886 \%$ \\
& 5 & 0.06661 & $4.040 \%$ & 0.06771 & $4.106 \%$ \\
& 6 & 0.03960 & $3.798 \%$ & 0.04004 & $3.841 \%$ \\
& 7 & 0.17395 & $4.452 \%$ & 0.17799 & $4.555 \%$ \\
& 8 & 0.11809 & $4.199 \%$ & 0.12034 & $4.279 \%$ \\
\hline \multirow{5}{6}{62} & 1 & 0.03951 & $3.747 \%$ & 0.03987 & $3.780 \%$ \\
& 2 & 0.04664 & $3.809 \%$ & 0.04712 & $3.849 \%$ \\
& 3 & 0.04537 & $3.800 \%$ & 0.04584 & $3.839 \%$ \\
& 4 & 0.04166 & $3.768 \%$ & 0.04206 & $3.804 \%$ \\
& 5 & 0.03180 & $3.661 \%$ & 0.03201 & $3.686 \%$ \\
& 6 & 0.04240 & $3.774 \%$ & 0.04281 & $3.810 \%$ \\
& 7 & 0.05673 & $3.879 \%$ & 0.05741 & $3.926 \%$ \\
& 8 & 0.04345 & $3.821 \%$ & 0.04386 & $3.857 \%$ \\
\hline \multirow{6}{*}{67} & 1 & 0.05092 & $3.726 \%$ & 0.05333 & $3.902 \%$ \\
& 2 & 0.04716 & $3.706 \%$ & 0.04927 & $3.871 \%$ \\
& 3 & 0.04872 & $3.715 \%$ & 0.05095 & $3.885 \%$ \\
& 4 & 0.04457 & $3.691 \%$ & 0.04646 & $3.848 \%$ \\
& 5 & 0.05210 & $3.733 \%$ & 0.05460 & $3.912 \%$ \\
& 6 & 0.04406 & $3.689 \%$ & 0.04591 & $3.845 \%$ \\
& 7 & 0.05171 & $3.732 \%$ & 0.05419 & $3.911 \%$ \\
& 8 & 0.02718 & $3.551 \%$ & 0.02776 & $3.626 \%$ \\
\hline
\end{tabular}

Table 5.24: Total systematic errors for each roadset data and for each $x_{2}$ bin. 


\subsubsection{Result of Cross section ratio}

The result of cross section ratio for each Roadset data is shown in Fig. 5.40.

\begin{tabular}{l|ll|llllll}
\hline \hline Roadset & $x_{2}$ Min & $x_{2}$ Max & $\left\langle x_{2}\right\rangle$ & $<$ Mass $>$ & $\sigma_{p d} / 2 \sigma_{p p}$ & Stat. err. & $\begin{array}{l}\text { Sys. err. } \\
\text { (lower) }\end{array}$ & $\begin{array}{l}\text { Sys. err. } \\
\text { (upper) }\end{array}$ \\
\hline \multirow{5}{*}{57} & 0.100 & 0.130 & 0.1200 & 4.3664 & 1.2375 & 0.1468 & 0.04807 & 0.04871 \\
& 0.130 & 0.160 & 0.1459 & 4.5141 & 1.1114 & 0.0691 & 0.04257 & 0.04308 \\
& 0.160 & 0.195 & 0.1765 & 4.7433 & 1.0862 & 0.0622 & 0.04150 & 0.04199 \\
& 0.195 & 0.240 & 0.2150 & 5.1343 & 1.1309 & 0.0746 & 0.04342 & 0.04394 \\
& 0.240 & 0.290 & 0.2618 & 5.6127 & 1.6488 & 0.3093 & 0.06661 & 0.06771 \\
& 0.290 & 0.350 & 0.3139 & 6.0441 & 1.0425 & 0.1351 & 0.03960 & 0.04004 \\
& 0.350 & 0.450 & 0.3874 & 6.7174 & 3.9075 & 3.6652 & 0.17395 & 0.17799 \\
& 0.450 & 0.580 & 0.4877 & 7.2806 & 2.8124 & 1.3414 & 0.11809 & 0.12034 \\
\hline \multirow{5}{*}{62} & 0.100 & 0.130 & 0.1200 & 4.3766 & 1.0545 & 0.0862 & 0.03951 & 0.03987 \\
& 0.130 & 0.160 & 0.1457 & 4.5150 & 1.2244 & 0.0667 & 0.04664 & 0.04712 \\
& 0.160 & 0.195 & 0.1766 & 4.7431 & 1.1941 & 0.0562 & 0.04537 & 0.04584 \\
& 0.195 & 0.240 & 0.2152 & 5.1434 & 1.1055 & 0.0587 & 0.04166 & 0.04206 \\
& 0.240 & 0.290 & 0.2623 & 5.6318 & 0.8686 & 0.0578 & 0.03180 & 0.03201 \\
& 0.290 & 0.350 & 0.3148 & 6.0980 & 1.1236 & 0.1123 & 0.04240 & 0.04281 \\
& 0.350 & 0.450 & 0.3885 & 6.6954 & 1.4625 & 0.2583 & 0.05673 & 0.05741 \\
& 0.450 & 0.580 & 0.4914 & 7.6415 & 1.1373 & 0.3873 & 0.04345 & 0.04386 \\
\hline \multirow{6}{*}{67} & 0.100 & 0.130 & 0.1200 & 4.3745 & 1.3667 & 0.0765 & 0.05092 & 0.05333 \\
& 0.130 & 0.160 & 0.1460 & 4.5079 & 1.2726 & 0.0415 & 0.04716 & 0.04927 \\
& 0.160 & 0.195 & 0.1767 & 4.7471 & 1.3115 & 0.0393 & 0.04872 & 0.05095 \\
& 0.195 & 0.240 & 0.2150 & 5.1342 & 1.2074 & 0.0395 & 0.04457 & 0.04646 \\
& 0.240 & 0.290 & 0.2622 & 5.5937 & 1.3957 & 0.0682 & 0.05210 & 0.05460 \\
& 0.290 & 0.350 & 0.3155 & 6.0810 & 1.1942 & 0.0722 & 0.04406 & 0.04591 \\
& 0.350 & 0.450 & 0.3841 & 6.6359 & 1.3855 & 0.1418 & 0.05171 & 0.05419 \\
& 0.450 & 0.580 & 0.4873 & 7.3891 & 0.7655 & 0.1465 & 0.02718 & 0.02776 \\
\hline \multirow{6}{*}{} & & & & & & & & \\
\end{tabular}

Table 5.25: Cross section ratio, $\sigma_{p d} / 2 \sigma_{p p}$, calculated from Roadset 57, 62 and 67 data for each $x_{2}$ bin. The average value of kinematic variables are also shown.

The three results of cross section ratio from the three Roadset data are then combined based on their statistical error to give an average cross section ratio $(R \pm \Delta)$ by:

$$
R \pm \Delta=\frac{\sum_{i} r_{i} / \delta_{i}^{2}}{\sum_{i} 1 / \delta_{i}^{2}} \pm\left\{\frac{1}{\sum_{i} 1 / \delta_{i}^{2}}\right\}^{1 / 2}
$$

where $r_{i}$ are cross section ratio of the three Roadset data, and $\delta_{i}$ are their statistical error. 


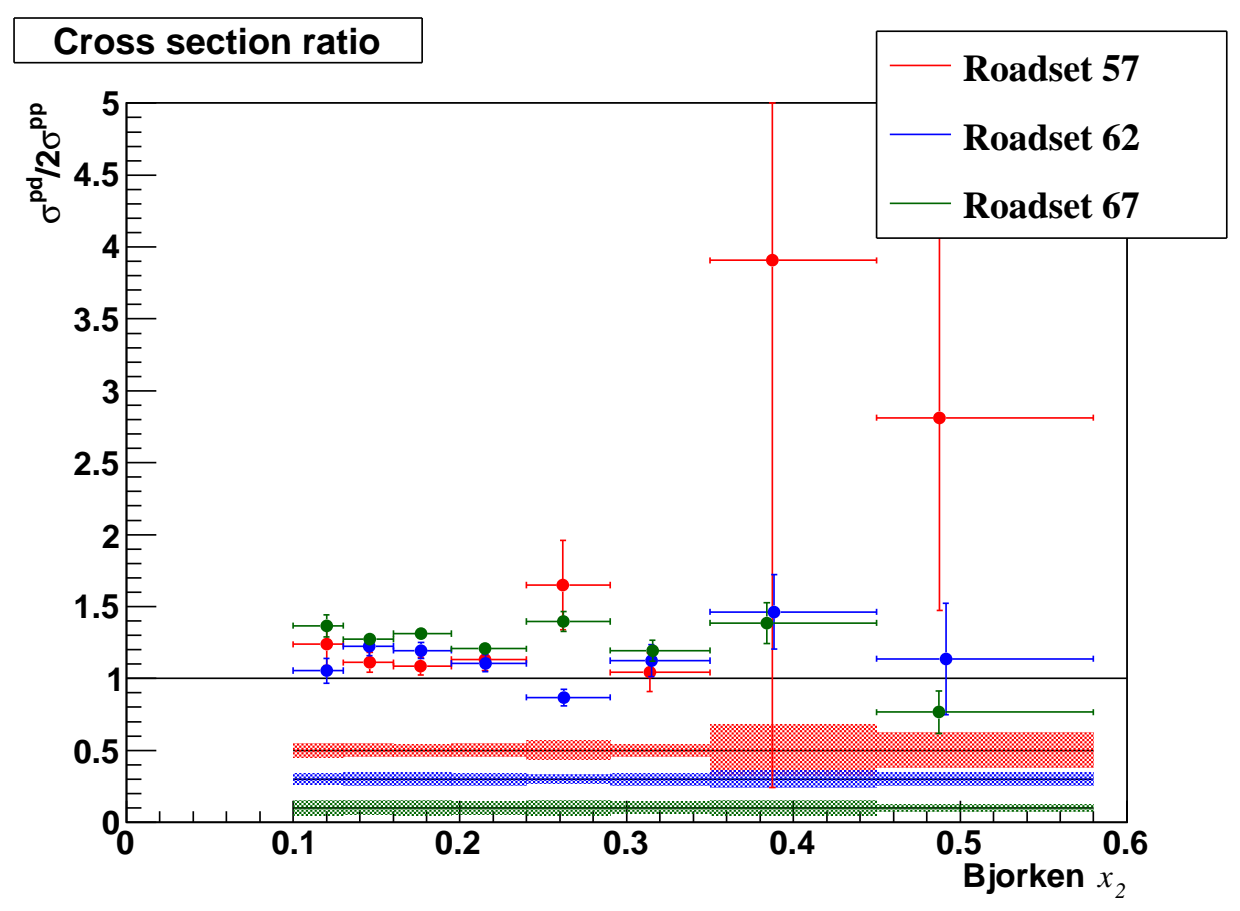

Figure 5.40: Cross section ratio versus Bjorken $x_{2}$ calculated from Roadset 57, 62 and 67 data. The Roadset 57 result is shown in red, the Roadset 62 result is shown in blue, and the Roadset 67 result is shown in green. The error bars represent the statistical error, and the error bands represent the systematic error. 


\begin{tabular}{lll|llllll}
\hline \hline Bin \# & $x_{2}$ Min & $x_{2} \operatorname{Max}$ & $\left\langle x_{2}\right\rangle$ & $\langle$ Mass $\rangle$ & $\sigma_{p d} / 2 \sigma_{p p}$ & Stat. err. & $\begin{array}{l}\text { Sys. err. } \\
\text { (lower) }\end{array}$ & (upper) \\
\hline 1 & 0.100 & 0.130 & 0.1200 & 4.374 & 1.230 & 0.0894 & 0.0462 & 0.0476 \\
3 & 0.130 & 0.160 & 0.1459 & 4.511 & 1.229 & 0.0528 & 0.0461 & 0.0475 \\
3 & 0.160 & 0.195 & 0.1766 & 4.745 & 1.233 & 0.0485 & 0.0463 & 0.0477 \\
4 & 0.195 & 0.240 & 0.2151 & 5.137 & 1.168 & 0.0502 & 0.0436 & 0.0449 \\
5 & 0.240 & 0.290 & 0.2623 & 5.616 & 1.100 & 0.0671 & 0.0408 & 0.0420 \\
6 & 0.290 & 0.350 & 0.3151 & 6.079 & 1.152 & 0.0925 & 0.0429 & 0.0442 \\
7 & 0.350 & 0.450 & 0.3851 & 6.650 & 1.406 & 0.1728 & 0.0530 & 0.0551 \\
8 & 0.450 & 0.580 & 0.4878 & 7.419 & 0.833 & 0.1886 & 0.0301 & 0.0307 \\
\hline
\end{tabular}

Table 5.26: The merged result of the cross section ratio using Eq. 5.36].

\section{Cross section ratio}

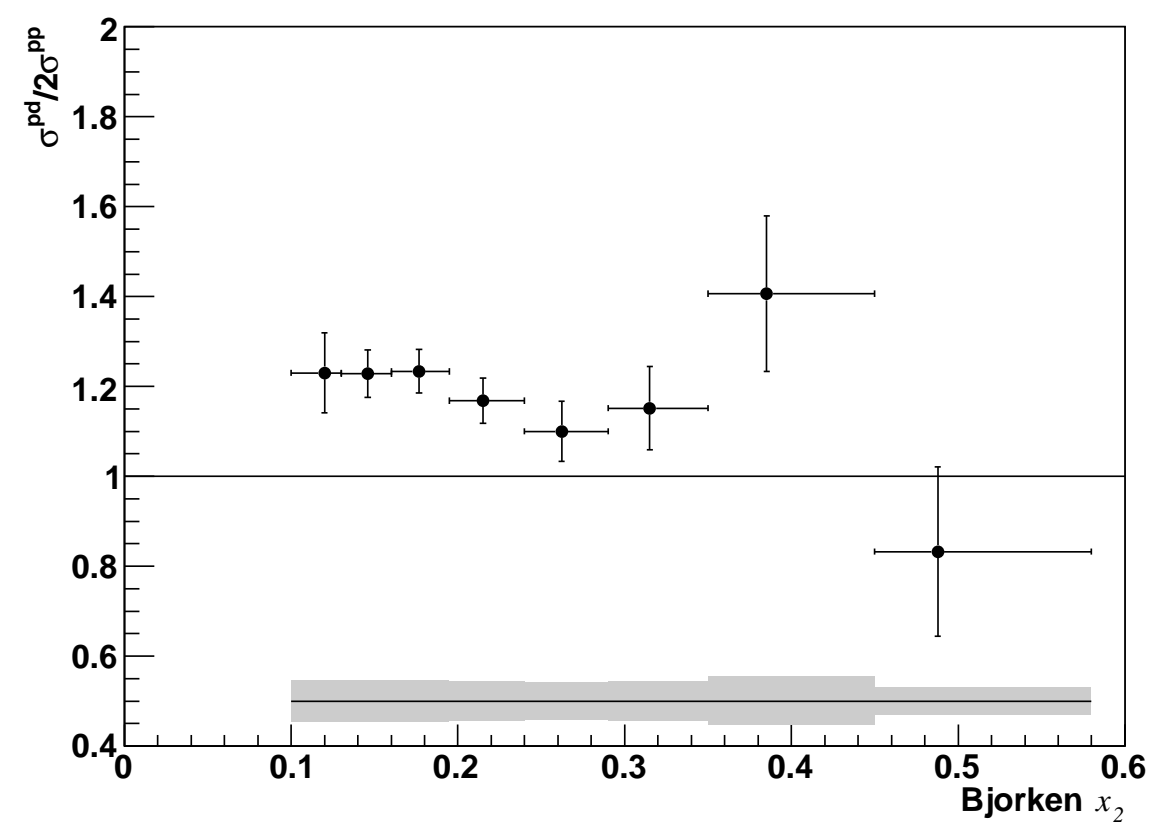

Figure 5.41: The merged result of the cross section ratio. The error bars represent the statistical error, and the error bands represents the systematic error. 


\subsection{Extraction of $\bar{d} / \bar{u}$}

The ratio $\bar{d} / \bar{u}$ is calculated using the cross section ratio obtained in previous sections. Although Eq. 3.13 well represents that the cross section ratio, $\sigma^{p d} / 2 \sigma^{p}$, is closely related to $\bar{d} / \bar{u}$, this equation is not used to extract the $\bar{d} / \bar{u}$. It is because that the equation uses several assumptions, and it is not accurate enough. Instead, an iterative process was used to extract the $\bar{d} / \bar{u}$ using $\sigma^{p d} / 2 \sigma^{p}$.

First, the cross section ratio is predicted in each iterative process using PDF parameterizations and current anticipated value of $\bar{d} / \bar{u}$. It is then compared with the result obtained from data. Next, the $\bar{d} / \bar{u}$ is adjusted to improve the agreement between the two cross section ratios. This iterative analysis continues until those cross sections match. More detailed procedure will be described in next subsections.

\subsubsection{Prediction of cross section ratio}

Prediction is done first for each dimuon pair obtained from LH2 and also LD2 target. Dimuon invariant mass, Bjorken $x_{1} \& x_{2}$ of the dimuon, and input $\bar{d} / \bar{u}$ are used to calculate the prediction. Formulas to calculate the cross sections, leading order of $\sigma^{p p} \&$ $\sigma^{p d}$, are defined by extending Eq. 3.8 as follows:

$$
\begin{aligned}
\sigma^{p p} \propto & 4 u\left(x_{1}\right) \bar{u}\left(x_{2}\right)+4 \bar{u}\left(x_{1}\right) u\left(x_{2}\right)+d\left(x_{1}\right) \bar{d}\left(x_{2}\right)+\bar{d}\left(x_{1}\right) d\left(x_{2}\right)+ \\
& 8 c\left(x_{1}\right) c\left(x_{2}\right)+2 s\left(x_{1}\right) s\left(x_{2}\right) \\
\sigma^{p n} \propto & 4 u\left(x_{1}\right) \bar{d}\left(x_{2}\right)+4 \bar{u}\left(x_{1}\right) d\left(x_{2}\right)+d\left(x_{1}\right) \bar{u}\left(x_{2}\right)+\bar{d}\left(x_{1}\right) u\left(x_{2}\right)+ \\
& 8 c\left(x_{1}\right) c\left(x_{2}\right)+2 s\left(x_{1}\right) s\left(x_{2}\right),
\end{aligned}
$$

where both have common coefficient, and then it is canceled when taking the ratio. The prediction of the cross section ratio for each dimuon event in data, $R_{i}$, is then derived by $\sigma^{p d} / 2 \sigma^{p d}=\left(\sigma^{p p}+\sigma^{p n}\right) / 2 \sigma^{p p}$. The value of $u(x), d(x), c(x)$ and $s(x)$ are taken from parton distribution function, PDF, based on the mass, $x_{1}$ and $x_{2}$ of each dimuon. CT10NLO [48] is used as the PDF. Also, sum of $\overline{u(x)}+\overline{d(x)}$ are taken. $\overline{u(x)}$ and $\overline{d(x)}$ are calculated using the sum and input $\overline{d(x)} / \overline{u(x)}$ as:

$$
\begin{aligned}
& \overline{u(x)}=\frac{(\overline{u(x)}+\overline{d(x)})_{\mathrm{pdf}}}{\left(\overline{\frac{d(x)}{u(x)}}\right)_{\text {input }}+1} \\
& \overline{d(x)}=\left(\frac{\overline{d(x)}}{\overline{u(x)}}\right)_{\text {input }} \cdot \overline{u(x)}
\end{aligned}
$$

Here, the following assumptions are made: 
- $c(x)=\overline{c(x)}, s(x)=\overline{s(x)}$

- contribution of heavier quarks, top and bottom quarks, are ignored due to their smaller probabilities.

- $\overline{d(x)} / \overline{u(x)}$ for the beam proton is the same as $\overline{d(x)} / \overline{u(x)}$ for the target proton over the $x_{2}$ range of the data

$x_{1}$ is larger than the maximum $x_{2}$ bin for many events. In that case, $\overline{d(x)} / \overline{u(x)}$ calculated using PDF was used for such large $x_{1}$. Finally, the mean value of all the predictions is taken as the prediction of the cross section ratio in this iteration, $R_{\mathrm{pr}}=\left\langle R_{i}\right\rangle$

\subsubsection{Adjustment of $\bar{d} / \bar{u}$}

The $\bar{d} / \bar{u}$ was adjusted to improve the agreement between the predicted cross section ratio, $R_{\mathrm{pr}}$, and obtained one from data, $R_{\mathrm{data}}$. Since $\bar{d} / \bar{u}$ is roughly proportional to the cross section ratio, $\sigma^{p d} / 2 \sigma^{p}$, as Eq. 3.13, the difference of $R_{\mathrm{pr}}$ and $R_{\text {data }}$ is added to the current $\bar{d} / \bar{u}$, and it is used for the next iteration step. The iteration analysis lasts until $\left|R_{\text {data }}-R_{\text {pr }}\right|<0.0000001$.

\subsubsection{Result of $\bar{d} / \bar{u}$}

\section{Statistical error}

In order to calculate the statistical error, $\bar{d} / \bar{u}$ extraction was done $2 n$ times more, where $n$ is the number of $x_{2}$ bin. For the first $n$ times, $R_{\text {data }}$ of one single bin was shifted upwards by an amount of its statistical error, while $R_{\text {data }}$ of the other bins stay the same. The next $n$ times are similar, except that $R_{\text {data }}$ of a bin was shifted downwards. The results of each of the $2 n$ extractions are compared to the original extraction of $\bar{d} / \bar{u}$, and the difference are taken as the errors. The Table. 5.27 shows the matrix of the statistical error, $r_{i j}$, which is the error on the bin $i$ when $R_{\text {data }}$ in the bin $j$ was shifted for Roadset 67 data as an example. Upper errors and lower errors are evaluated, respectively.

After the error matrix is made this way, the statistical error on the bin $i$ is finally calculated as:

$$
\delta_{i}=\sqrt{\sum_{j} r_{i j}^{2}} .
$$

Contribution of the shifting of $R_{\text {data }}$ in its own bin is a large portion of the final error, and that from the other bins is almost negligible as can be seen in the matrix table. 


\begin{tabular}{llllllllll}
\hline \hline & 1 & 2 & 3 & 4 & 5 & 6 & 7 & 8 \\
\hline & 1 & $1.985 \mathrm{e}-01$ & $1.561 \mathrm{e}-07$ & $1.676 \mathrm{e}-07$ & $1.676 \mathrm{e}-07$ & $1.676 \mathrm{e}-07$ & $1.676 \mathrm{e}-07$ & $1.651 \mathrm{e}-07$ & $3.963 \mathrm{e}-06$ \\
& 2 & $1.797 \mathrm{e}-07$ & $1.062 \mathrm{e}-01$ & $1.849 \mathrm{e}-07$ & $1.797 \mathrm{e}-07$ & $1.797 \mathrm{e}-07$ & $1.942 \mathrm{e}-05$ & $9.730 \mathrm{e}-05$ & $3.034 \mathrm{e}-03$ \\
67 & 3 & $1.788 \mathrm{e}-06$ & $1.788 \mathrm{e}-06$ & $1.087 \mathrm{e}-01$ & $1.808 \mathrm{e}-06$ & $1.788 \mathrm{e}-06$ & $1.048 \mathrm{e}-04$ & $3.440 \mathrm{e}-04$ & $1.611 \mathrm{e}-02$ \\
Upper & 4 & $3.045 \mathrm{e}-06$ & $3.045 \mathrm{e}-06$ & $3.045 \mathrm{e}-06$ & $1.114 \mathrm{e}-01$ & $3.072 \mathrm{e}-06$ & $1.453 \mathrm{e}-04$ & $5.363 \mathrm{e}-04$ & $2.190 \mathrm{e}-02$ \\
& 5 & $1.574 \mathrm{e}-05$ & $1.574 \mathrm{e}-05$ & $1.574 \mathrm{e}-05$ & $1.574 \mathrm{e}-05$ & $2.391 \mathrm{e}-01$ & $4.012 \mathrm{e}-04$ & $5.865 \mathrm{e}-03$ & $5.793 \mathrm{e}-02$ \\
& 6 & $1.723 \mathrm{e}-05$ & $1.723 \mathrm{e}-05$ & $1.723 \mathrm{e}-05$ & $1.723 \mathrm{e}-05$ & $1.723 \mathrm{e}-05$ & $2.586 \mathrm{e}-01$ & $9.853 \mathrm{e}-03$ & $5.808 \mathrm{e}-02$ \\
& 7 & $1.047 \mathrm{e}-04$ & $1.047 \mathrm{e}-04$ & $1.047 \mathrm{e}-04$ & $1.047 \mathrm{e}-04$ & $1.047 \mathrm{e}-04$ & $3.133 \mathrm{e}-04$ & $8.807 \mathrm{e}-01$ & $2.253 \mathrm{e}-01$ \\
& 8 & $7.244 \mathrm{e}-06$ & $7.245 \mathrm{e}-06$ & $7.245 \mathrm{e}-06$ & $7.245 \mathrm{e}-06$ & $7.245 \mathrm{e}-06$ & $2.497 \mathrm{e}-03$ & $1.240 \mathrm{e}-02$ & $5.262 \mathrm{e}-01$ \\
\hline & 1 & $1.939 \mathrm{e}-01$ & $1.676 \mathrm{e}-07$ & $1.676 \mathrm{e}-07$ & $1.676 \mathrm{e}-07$ & $1.676 \mathrm{e}-07$ & $1.676 \mathrm{e}-07$ & $6.612 \mathrm{e}-06$ & $1.535 \mathrm{e}-07$ \\
& 2 & $1.797 \mathrm{e}-07$ & $1.048 \mathrm{e}-01$ & $1.797 \mathrm{e}-07$ & $1.797 \mathrm{e}-07$ & $1.797 \mathrm{e}-07$ & $1.954 \mathrm{e}-05$ & $1.135 \mathrm{e}-04$ & $4.857 \mathrm{e}-03$ \\
67 & 3 & $1.788 \mathrm{e}-06$ & $1.788 \mathrm{e}-06$ & $1.069 \mathrm{e}-01$ & $1.788 \mathrm{e}-06$ & $1.788 \mathrm{e}-06$ & $1.037 \mathrm{e}-04$ & $3.785 \mathrm{e}-04$ & $2.603 \mathrm{e}-02$ \\
Lower & 4 & $3.045 \mathrm{e}-06$ & $3.045 \mathrm{e}-06$ & $3.045 \mathrm{e}-06$ & $1.092 \mathrm{e}-01$ & $3.045 \mathrm{e}-06$ & $1.426 \mathrm{e}-04$ & $7.171 \mathrm{e}-04$ & $3.536 \mathrm{e}-02$ \\
& 5 & $1.574 \mathrm{e}-05$ & $1.574 \mathrm{e}-05$ & $1.574 \mathrm{e}-05$ & $1.574 \mathrm{e}-05$ & $2.287 \mathrm{e}-01$ & $3.782 \mathrm{e}-04$ & $7.033 \mathrm{e}-03$ & $9.284 \mathrm{e}-02$ \\
& 6 & $1.723 \mathrm{e}-05$ & $1.723 \mathrm{e}-05$ & $1.723 \mathrm{e}-05$ & $1.723 \mathrm{e}-05$ & $1.723 \mathrm{e}-05$ & $2.433 \mathrm{e}-01$ & $1.171 \mathrm{e}-02$ & $9.143 \mathrm{e}-02$ \\
& 7 & $1.047 \mathrm{e}-04$ & $1.047 \mathrm{e}-04$ & $1.047 \mathrm{e}-04$ & $1.047 \mathrm{e}-04$ & $1.047 \mathrm{e}-04$ & $9.291 \mathrm{e}-05$ & $7.411 \mathrm{e}-01$ & $3.470 \mathrm{e}-01$ \\
& 8 & $7.245 \mathrm{e}-06$ & $7.245 \mathrm{e}-06$ & $7.245 \mathrm{e}-06$ & $7.245 \mathrm{e}-06$ & $7.245 \mathrm{e}-06$ & $2.551 \mathrm{e}-03$ & $1.412 \mathrm{e}-02$ & $4.470 \mathrm{e}-01$ \\
\hline
\end{tabular}

Table 5.27: Matrix table of $r_{i j}$, which is the error on the bin $i$ when $R_{\text {data }}$ in the bin $j$ was adjusted for Roadset 67 data. 


\section{Systematic error}

The systematic error on $\bar{d} / \bar{u}$ ratio is calculated using the systematic and statistical error of cross section ratio and $\bar{d} / \bar{u}$ ratio as follows:

$$
\delta_{\bar{d} / \bar{u}}=\frac{\Delta_{\bar{d} / \bar{u}}}{\Delta_{\text {CSratio }}} \cdot \delta_{\text {CSratio }}
$$

where $\delta$ indicates systematic error and $\Delta$ indicates statistical error. In addition to this systematic error $\delta_{\bar{d} / \bar{u}}$, one more systematic error is checked. It is related to the PDF parameterizations used when obtaining $\bar{d} / \bar{u}$. For the SeaQuest result, CT10NLO is used while CTEQ5M was used for the E866 result [14]. Figure 5.42 shows PDFs of CT10NLO and CTEQ5M, and their ratios. They are different especially at higher $x$. Due to the difference, the $\bar{d} / \bar{u}$ result could vary if CTEQ5M is used in the analysis instead of CT10NLO.

$\bar{d} / \bar{u}$ is evaluated from the SeaQuest data using CTEQ5M as well in order to estimate the systematic error due to the choice of PDF parameterizations. The result is shown in Fig. 5.43. The result with CT10NLO is drawn in red and the result with CTEQ5M is drawn in black. The difference between the two results is small, and it is $1.3 \%$. The total systematic error is then calculated as:

$$
\delta_{\mathrm{tot}}=\sqrt{\delta^{2}+\delta_{\mathrm{PDF}}^{2}}
$$

where $\delta$ is the systematic error derived by Eq. 5.42 and $\delta_{\mathrm{PDF}}$ is the systematic error due to the choice of PDF parameterizations. 

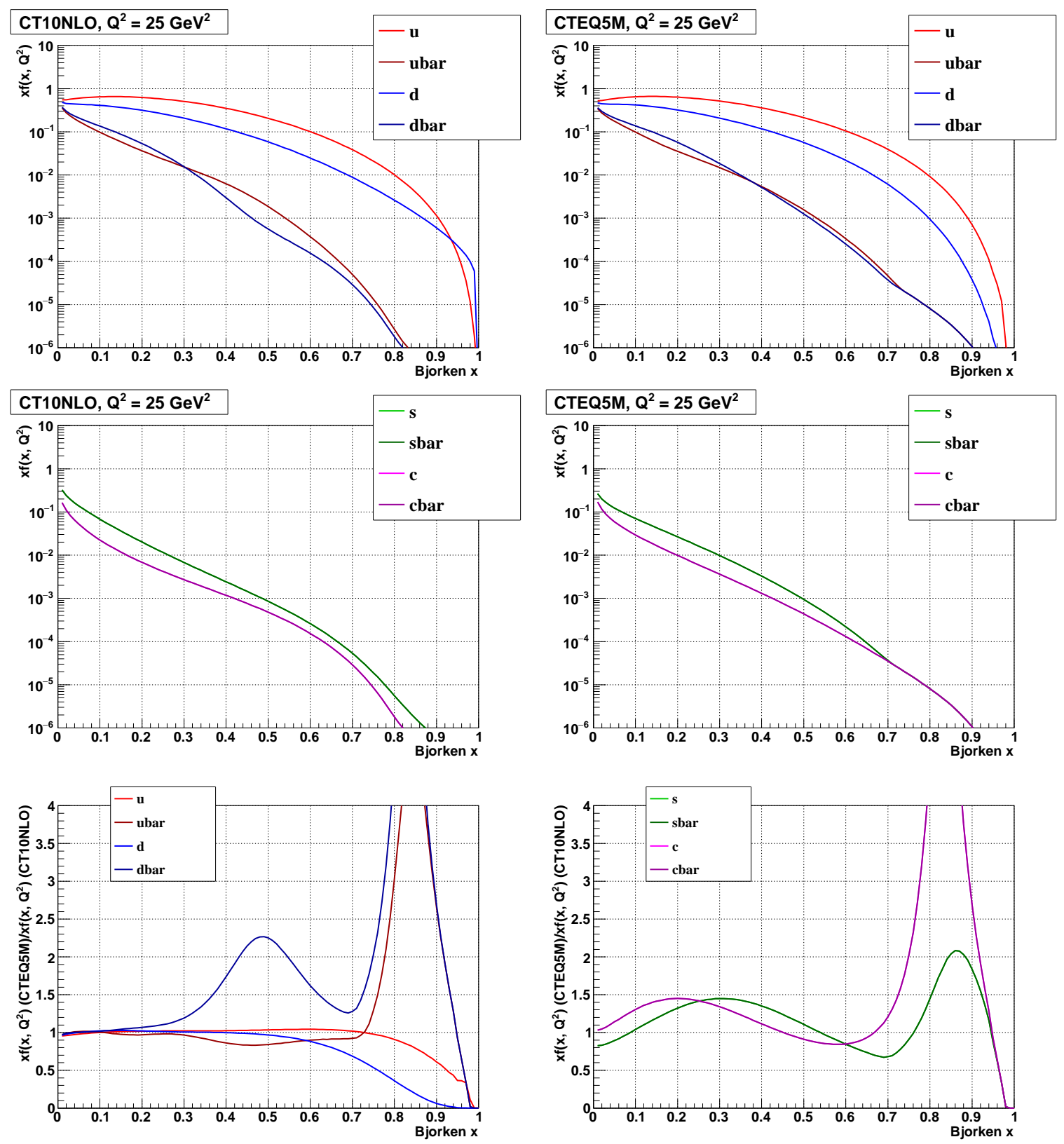

Figure 5.42: PDFs of CT10NLO and CTEQ5M. The top two figures show $u, d, \bar{u}$, and $\bar{d}$ of CT10NLO(left) and CTEQ5M(right). The middle two figures show $s, c, \bar{s}$, and $\bar{c}$ of CT10NLO(left) and CTEQ5M(right). The bottom two figures show their ratios (CTEQ5M/CT10NLO). 


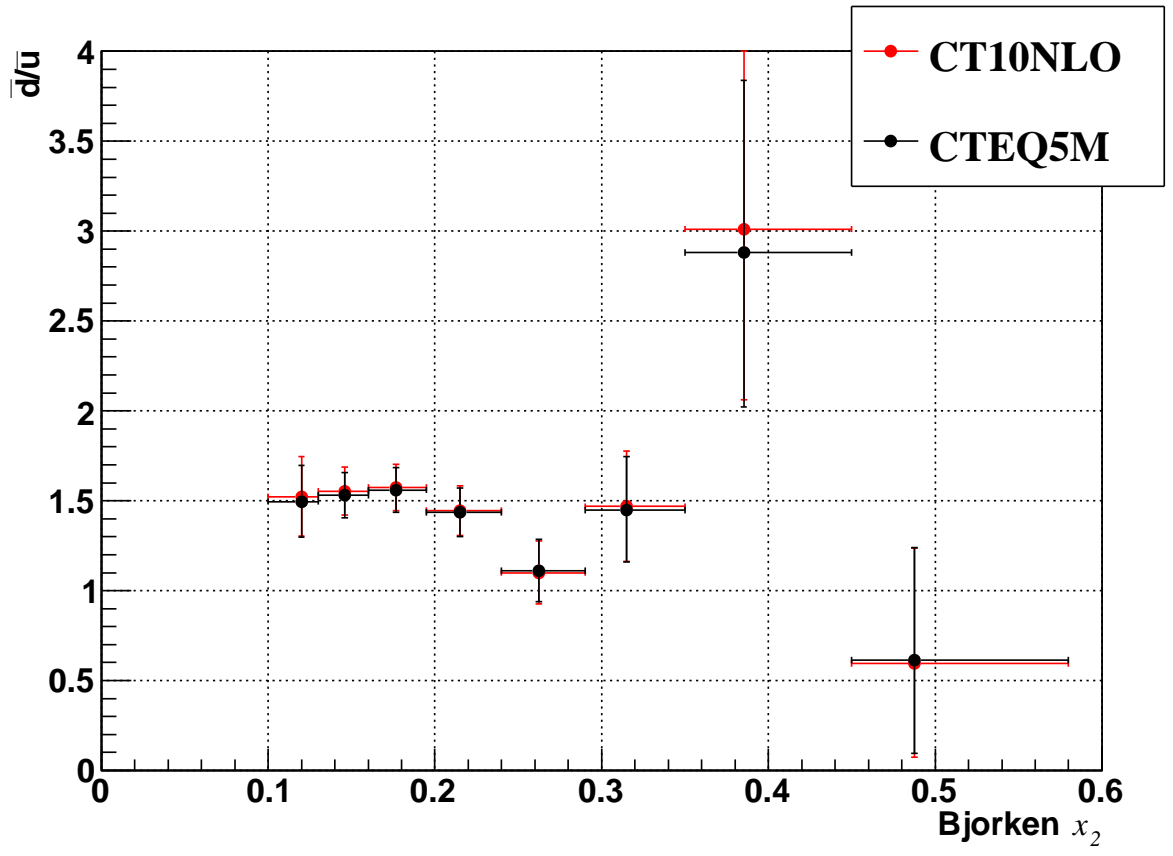

Figure 5.43: $\quad \bar{d} / \bar{u}$ results using two different PDF parameterizations. The result with CT10NLO is drawn in red and the that with CTEQ5M is drawn in black. 


\section{Result}

The result of $\bar{d} / \bar{u}$ for each Roadset data is shown in Fig. $\$ 5.44$ and listed in Table. $\$ 5.28$. These results are merged using Eq. 5.36 as was done for cross section ratio. The result is shown in Fig. 5.45 and the values are listed in Table. 5.2.9.

\begin{tabular}{l|ll|l|ll|ll}
\hline \hline Roadset & x Min & x Max & $\bar{d} / \bar{u}$ & \multicolumn{2}{|l|}{ Stat. error } & & \multicolumn{2}{l}{ Sys. error } \\
(lower) & (upper) & (lower) & (upper) \\
\hline \multirow{5}{*}{57} & 0.100 & 0.130 & 1.5648 & 0.3522 & 0.3676 & 0.1172 & 0.1237 \\
& 0.130 & 0.160 & 1.2669 & 0.1635 & 0.1670 & 0.1021 & 0.1055 \\
& 0.160 & 0.195 & 1.2039 & 0.1480 & 0.1507 & 0.1001 & 0.1030 \\
& 0.195 & 0.240 & 1.3120 & 0.1850 & 0.1871 & 0.1090 & 0.1115 \\
& 0.240 & 0.290 & 2.7669 & 0.9376 & 1.0467 & 0.2052 & 0.2320 \\
& 0.290 & 0.350 & 1.0698 & 0.3628 & 0.3439 & 0.1073 & 0.1029 \\
& 0.350 & 0.450 & 37.717 & 51.129 & 295.78 & 2.4771 & 14.373 \\
& 0.450 & 0.580 & 17.830 & 22.253 & 65.341 & 1.9732 & 5.8668 \\
\hline \multirow{5}{*}{62} & 0.100 & 0.130 & 1.1190 & 0.1984 & 0.2034 & 0.0921 & 0.0953 \\
& 0.130 & 0.160 & 1.5464 & 0.1646 & 0.1680 & 0.1168 & 0.1204 \\
& 0.160 & 0.195 & 1.4867 & 0.1436 & 0.1451 & 0.1175 & 0.1199 \\
& 0.195 & 0.240 & 1.2806 & 0.1511 & 0.1524 & 0.1085 & 0.1104 \\
& 0.240 & 0.290 & 0.7032 & 0.1367 & 0.1394 & 0.0758 & 0.0778 \\
& 0.290 & 0.350 & 1.3988 & 0.3310 & 0.3453 & 0.1263 & 0.1329 \\
& 0.350 & 0.450 & 2.9717 & 1.2748 & 1.4791 & 0.2827 & 0.3311 \\
& 0.450 & 0.580 & 1.9261 & 1.4700 & 2.0648 & 0.1669 & 0.2352 \\
\hline \multirow{5}{*}{67} & 0.100 & 0.130 & 1.8936 & 0.1939 & 0.1985 & 0.1315 & 0.1407 \\
& 0.130 & 0.160 & 1.6721 & 0.1049 & 0.1063 & 0.1211 & 0.1279 \\
& 0.160 & 0.195 & 1.8245 & 0.1101 & 0.1099 & 0.1386 & 0.1445 \\
& 0.195 & 0.240 & 1.5884 & 0.1148 & 0.1135 & 0.1312 & 0.1351 \\
& 0.240 & 0.290 & 2.2619 & 0.2469 & 0.2461 & 0.1908 & 0.1992 \\
& 0.290 & 0.350 & 1.7306 & 0.2602 & 0.2652 & 0.1604 & 0.1702 \\
& 0.350 & 0.450 & 3.0257 & 0.8184 & 0.9091 & 0.3010 & 0.3496 \\
& 0.450 & 0.580 & 0.4928 & 0.4472 & 0.5264 & 0.0833 & 0.1000 \\
\hline
\end{tabular}

Table 5.28: Result of $\bar{d} / \bar{u}$ extracted from Roadset 57, 62 and 67 data. 


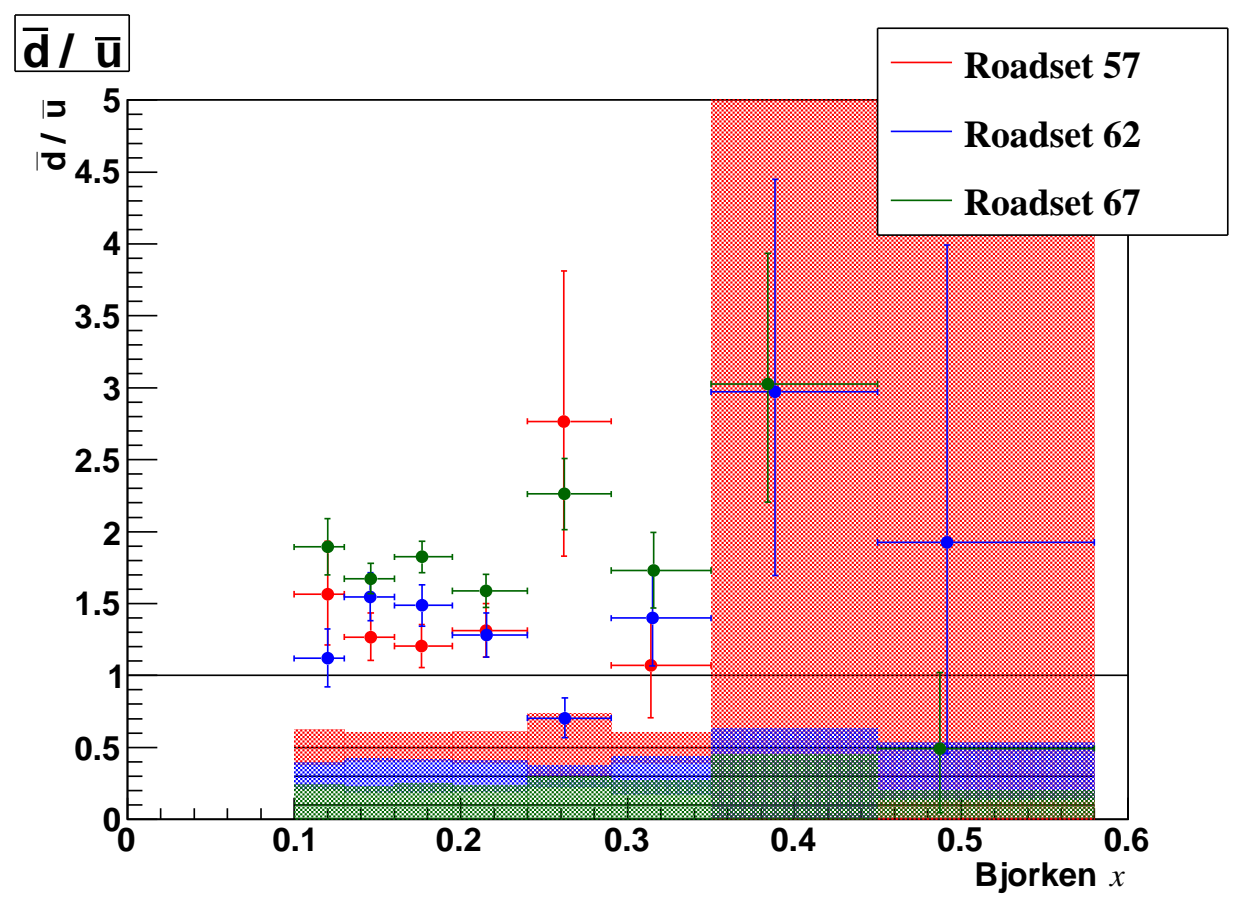

Figure 5.44: Result of $\bar{d} / \bar{u}$ extracted from Roadset 57, 62 and 67 data. The Roadset 57 result is shown in red, the Roadset 62 result is shown in blue, and the Roadset 67 result is shown in green. The error bars represent the statistical error, and the error bands represents the systematic error. 


\begin{tabular}{lll|l|ll|ll}
\hline \hline Bin \# & $\mathrm{x}$ Min & $\mathrm{x}$ Max & $\bar{d} / \bar{u}$ & $\begin{array}{l}\text { Stat. error } \\
\text { (lower) }\end{array}$ & (upper) & $\begin{array}{l}\text { Sys. error } \\
\text { (lower) }\end{array}$ & (upper) \\
\hline 1 & 0.100 & 0.130 & 1.5220 & 0.2167 & 0.2229 & 0.1111 & 0.1175 \\
3 & 0.130 & 0.160 & 1.5528 & 0.1314 & 0.1337 & 0.1140 & 0.1195 \\
3 & 0.160 & 0.195 & 1.5736 & 0.1289 & 0.1299 & 0.1212 & 0.1255 \\
4 & 0.195 & 0.240 & 1.4452 & 0.1390 & 0.1391 & 0.1188 & 0.1219 \\
5 & 0.240 & 0.290 & 1.0999 & 0.1742 & 0.1777 & 0.1037 & 0.1076 \\
6 & 0.290 & 0.350 & 1.4683 & 0.3063 & 0.3078 & 0.1358 & 0.1412 \\
7 & 0.350 & 0.450 & 3.0111 & 0.9481 & 1.0753 & 0.2932 & 0.3423 \\
8 & 0.450 & 0.580 & 0.5959 & 0.5218 & 0.6423 & 0.0890 & 0.1099 \\
\hline
\end{tabular}

Table 5.29: The merged result of $\bar{d} / \bar{u}$ using Eq .5.36.

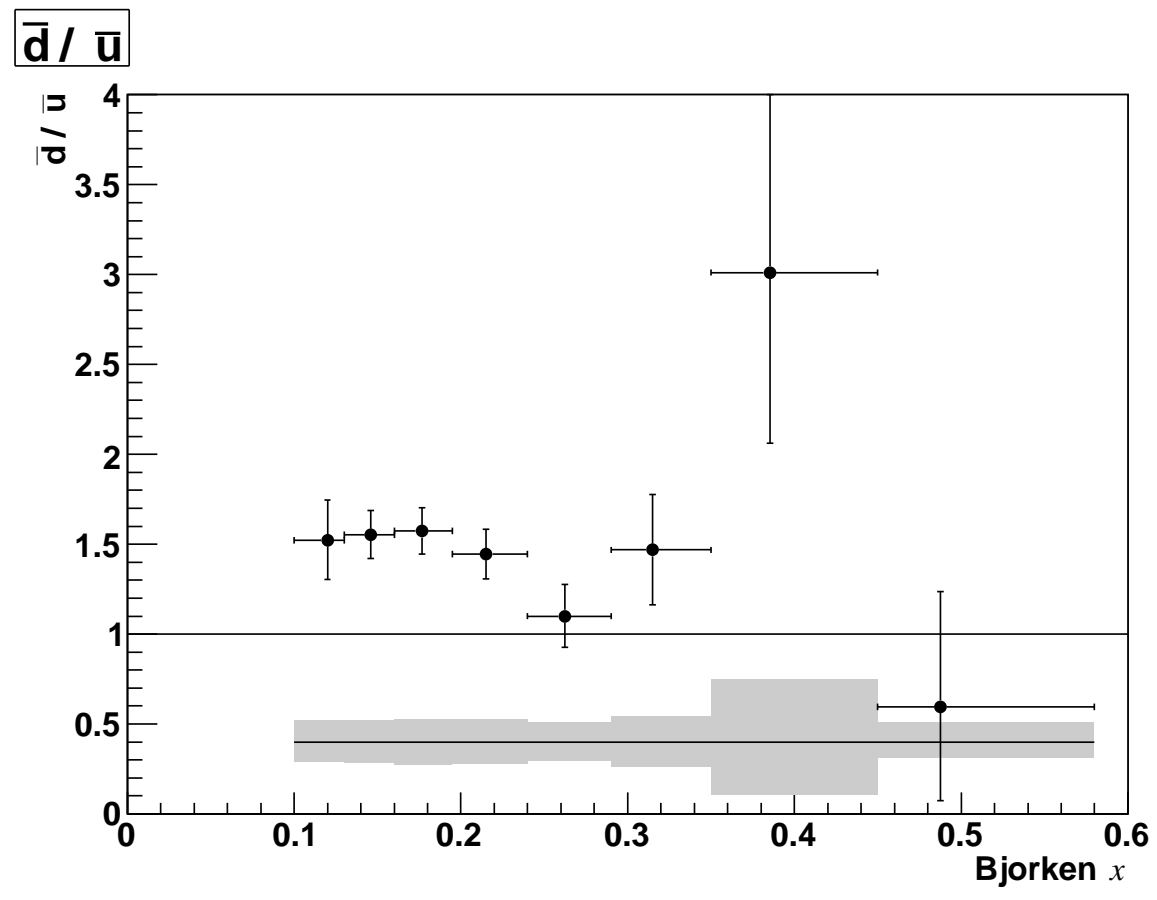

Figure 5.45: The merged result of $\bar{d} / \bar{u}$. The error bars represent the statistical error, and the error band represents the systematic error. 


\section{Chapter 6}

\section{Discussion of the Results}

Following the NMC result, the NA51 experiment and the E866 experiment indicated the flavor asymmetry in the proton prior to the SeaQuest experiment. In this chapter, the results of the previous experiments, several PDF parameterizations, and non-perturbative models are compared to the SeaQuest results. The cross section ratio is discussed in section 6.7 and then anti-quark flavor asymmetry is discussed in section 6.2 .

\subsection{Cross section ratio}

The SeaQuest result of cross section ratio is compared to the E866 result as shown in Fig.6.]. The SeaQuest result is plotted in red and the E866 result is plotted in black. The error bars represent the statistical uncertainty, and the error band at the bottom represents the systematic uncertainty. The cross section ratio is successfully measured at high Bjorken $x$ (up to $x=0.58$ ) in the SeaQuest experiment as was designed, which is much wider region than the E866 measurement. For values of $x$ of 0.3 and lower, the SeaQuest points are slightly higher than the E866 points. There is a physical reason for it as will be discussed later in this thesis. For values of $x$ of 0.3 and higher, the SeaQuest points keep over 1 till $x=0.45$ while the E866 points goes below 1 at $x=0.3$.

Both are compared to the prediction calculated with PDF parameterization of CT10NLO[48] in the bottom figure of Fig.6.]. In each bin, the cross section ratio was calculated as Eq. 5.38 using the parameterizations with the averaged value of mass, $x_{1}$ and $x_{2}$. The points are then smoothly connected.

The averaged kinematic values are listed for SeaQuest in Table. 6.], and for E866 in Table. 6.2. Figure 6.2 shows the averaged kinematic values as a function of $\left\langle x_{2}\right\rangle$. Left figure shows $\left\langle x_{1}>\right.$ vs $<x_{2}>$ for SeaQuest (red) and E866 (black). Right figure shows $<$ Mass $>$ vs $<x_{2}>$ for SeaQuest (red) and E866 (black). It can be seen that 

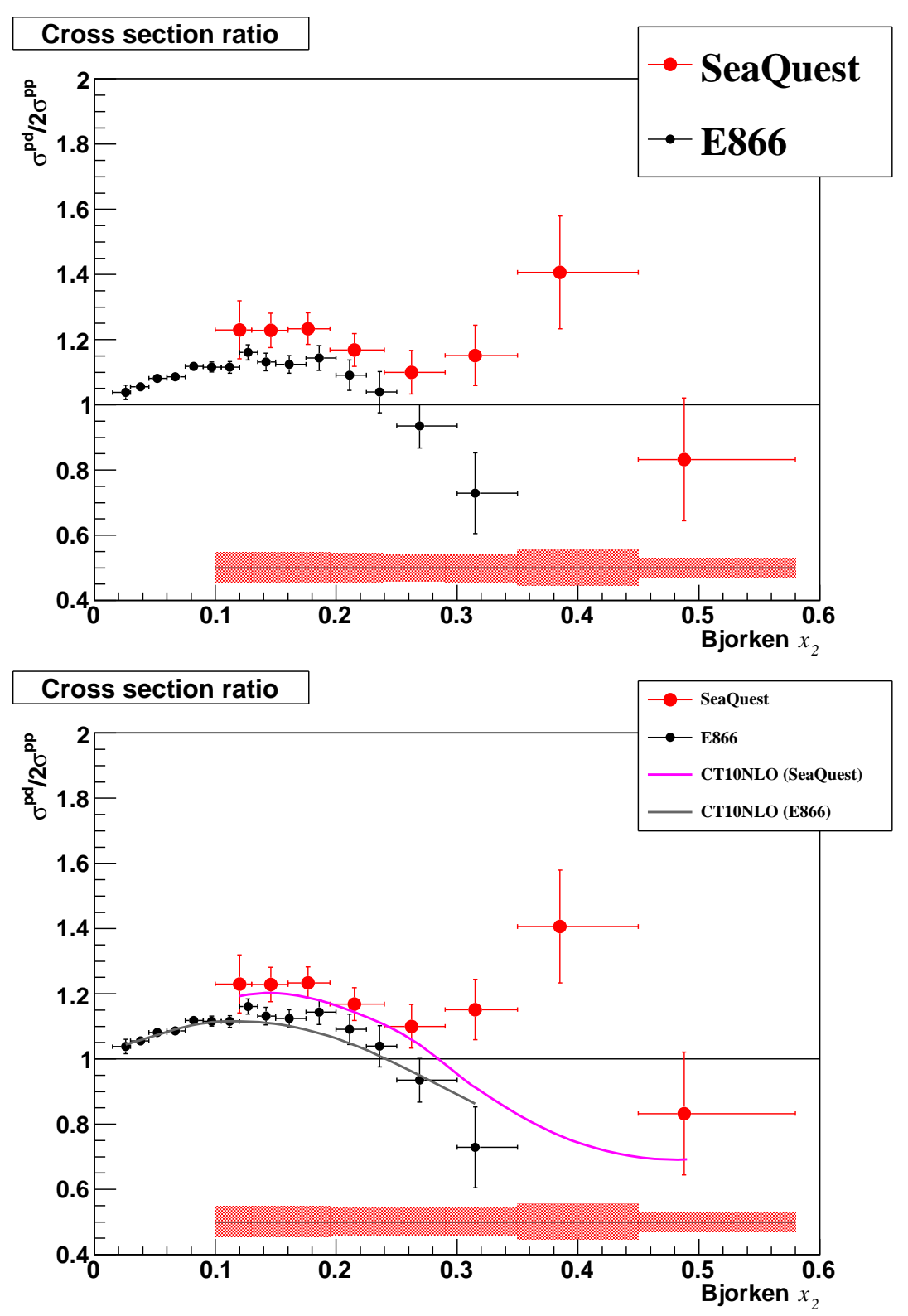

Figure 6.1: Cross section ratio as a function of Bjorken $x$. The SeaQuest result is plotted in red and the E866 result is plotted in black. The error bars represent the statistical uncertainty, and the error band at the bottom represent the systematic uncertainty. The position of the error band was arbitrary chosen. The systematic uncertainty of E866 is less than $1 \%$ and is not shown. In the bottom figure, also the predictions calculated with CT10NLO PDF parameterizations for SeaQuest (magenta line) and E866 (gray line) are drawn. 
$<x_{1}>$ distribution of SeaQuest is higher than that of E866, and $<$ Mass $>$ distribution of SeaQuest is lower than that of E866. $\left\langle x_{1}>\right.$ is the Bjorken $x$ of the beam proton.

\begin{tabular}{ll|lll}
\hline \hline$x_{2} \operatorname{Min}$ & $x_{2} \operatorname{Max}$ & $\left\langle x_{1}\right\rangle$ & $\left\langle x_{2}\right\rangle$ & $\langle$ Mass $\rangle\left(\mathrm{GeV}^{2}\right)$ \\
\hline 0.100 & 0.130 & 0.7039 & 0.1200 & 4.3742 \\
0.130 & 0.160 & 0.6023 & 0.1459 & 4.5108 \\
0.160 & 0.195 & 0.5518 & 0.1766 & 4.7450 \\
0.195 & 0.240 & 0.5403 & 0.2151 & 5.1369 \\
0.240 & 0.290 & 0.5854 & 0.2623 & 5.6225 \\
0.290 & 0.350 & 0.5164 & 0.3149 & 6.0765 \\
0.350 & 0.450 & 0.4679 & 0.3853 & 6.6526 \\
0.450 & 0.580 & 0.5882 & 0.4876 & 7.4069 \\
\hline
\end{tabular}

Table 6.1: The averaged values for kinematic variables of the SeaQuest experiment.

\begin{tabular}{ll|lll}
\hline \hline$x_{2} \operatorname{Min}$ & $x_{2} \operatorname{Max}$ & $\left\langle x_{1}\right\rangle$ & $\left\langle x_{2}\right\rangle$ & $\langle$ Mass $\rangle\left(\mathrm{GeV}^{2}\right)$ \\
\hline 0.015 & 0.030 & 0.559 & 0.026 & 4.6 \\
0.030 & 0.045 & 0.454 & 0.038 & 5.1 \\
0.045 & 0.060 & 0.408 & 0.052 & 5.6 \\
0.060 & 0.075 & 0.393 & 0.067 & 6.2 \\
0.075 & 0.090 & 0.378 & 0.082 & 6.8 \\
0.090 & 0.105 & 0.358 & 0.097 & 7.2 \\
0.105 & 0.120 & 0.339 & 0.112 & 7.5 \\
0.120 & 0.135 & 0.326 & 0.127 & 7.8 \\
0.135 & 0.150 & 0.324 & 0.142 & 8.2 \\
0.150 & 0.175 & 0.325 & 0.161 & 8.7 \\
0.175 & 0.200 & 0.333 & 0.186 & 9.5 \\
0.200 & 0.225 & 0.345 & 0.211 & 10.3 \\
0.225 & 0.250 & 0.356 & 0.236 & 11.1 \\
0.250 & 0.300 & 0.366 & 0.269 & 12.0 \\
0.300 & 0.350 & 0.361 & 0.315 & 12.9 \\
\hline
\end{tabular}

Table 6.2: The averaged values for kinematic variables of the E866 experiment [14].

The magenta line shows the line of the prediction using the SeaQuest kinematics and the gray line shows the that using the E866 kinematics. The prediction curve is in reasonable agreement with the SeaQuest results for $x$ of 0.3 and lower. Difference of $\sqrt{s}$ makes the difference of mass and $x_{1}$ for the same $x_{2}$ range between the two experiments. The $x_{1}$ difference mainly contributes to the difference of those two prediction lines because the value of PDF doesn't vary by mass so much as can be seen in Fig. [6.3. 

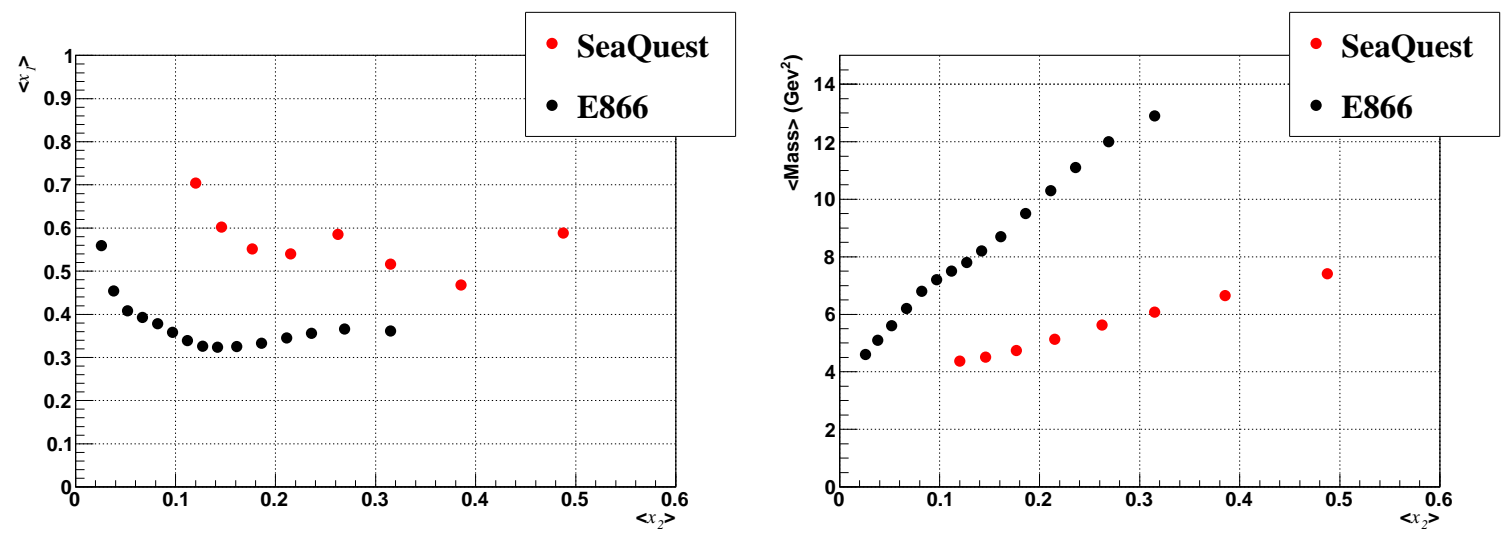

Figure 6.2: Left figure shows $\left\langle x_{1}>\right.$ vs $<x_{2}>$ for SeaQuest (red) and E866 (black). Right figure shows $<$ Mass $>$ vs $<x_{2}>$ for SeaQuest (red) and E866 (black).

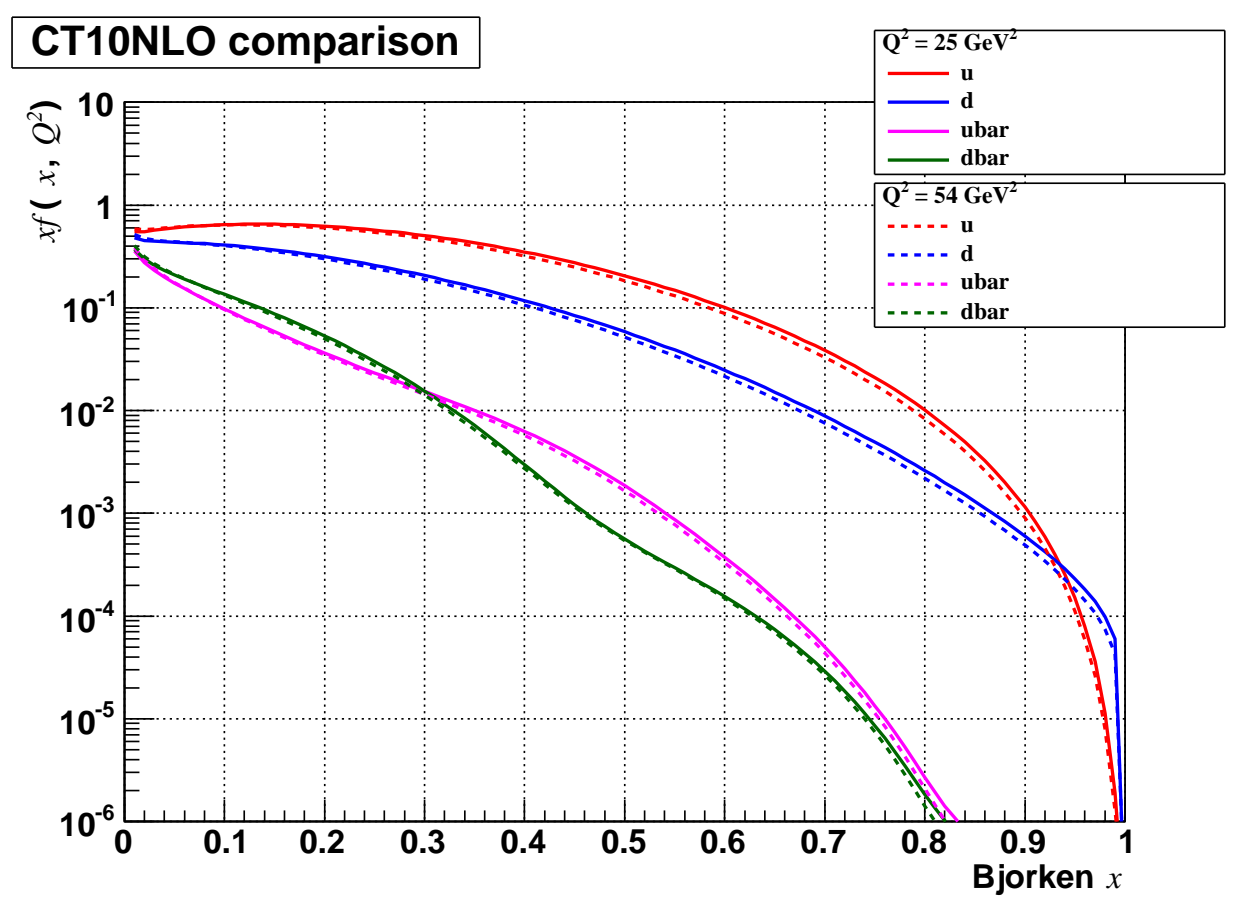

Figure 6.3: Parton distribution function at scale of $Q^{2}=25 \mathrm{GeV}^{2}$ (solid line) and $Q^{2}=54 \mathrm{GeV}^{2}$ (dot line). These values are averaged $Q^{2}$ value of SeaQuest and E866, respectively. $\bar{d}$ is larger than $\bar{u}$ at $x<0.3$, and $\bar{u}$ takes over at $x>0.3$ in this parameterization. 


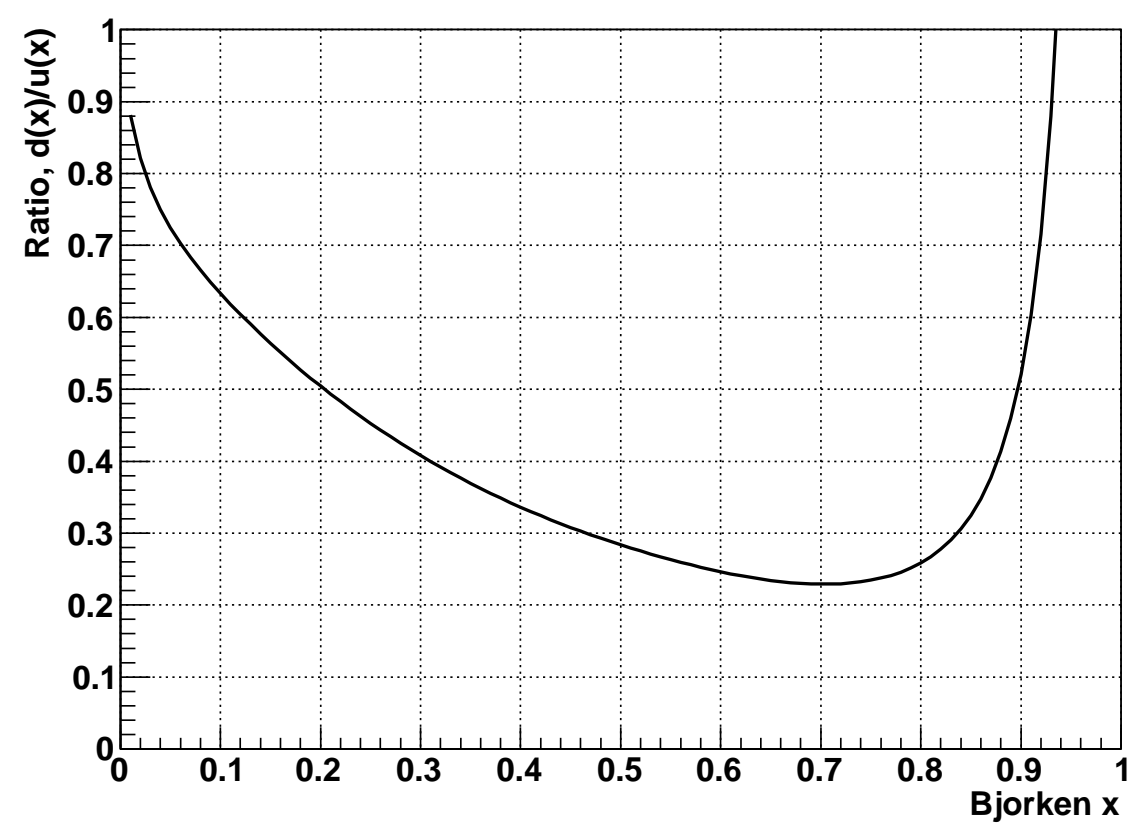

Figure 6.4: Ratio of $d(x) / u(x)$ as a function of $x$. The ratio of $d(x) / u(x)$ decreases as $x$ increases until 0.7 .

According to Eq. [.]2, if $d(x) / u(x)$ is smaller, the cross section ratio becomes larger. Figure. 6.4 shows the ratio of $d(x) / u(x)$ as a function of $x$. The ratio of $d(x) / u(x)$ decreases as $x$ increases until 0.7 as shown in the figure. Therefore, it is reasonable that the SeaQuest points are higher than the E866 results. 


\subsection{Flavor asymmetry}

\subsubsection{Comparison to other experiments}

The $\bar{d} / \bar{u}$ result is compared with the E866 result and the NA51 result in Fig. 6.5 . The SeaQuest result is plotted in red, the E866 result in black, and the NA51 result in gray. The error bars represent the statistical uncertainty, and the error band at bottom represent the systematic uncertainty. The difference between the SeaQuest and E866 results is less distinct than in the cross section ratio plot (Fig.6. D).

This feature is reproduced by the prediction using CT10NLO PDF as can be seen in the bottom figure of Fig. 6.5. The gap between the two predictions become much closer than that in the cross section ratio plot. It is because that the ratio of $\bar{d} / \bar{u}$ is a function of $x_{2}$ and mass while the cross section ratio is a function of $x_{1}, x_{2}$ and mass. The mass difference between the two experiment doesn't affect the PDF form as already shown in Fig. 6.3. So, the difference in $\left\langle x_{1}\right\rangle$ makes a large effect in the cross section ratio. For the $x=0.3$ and higher, there is a large difference in the ratio $\bar{d} / \bar{u}$ between the two experimental results.

Fig. 6.6 shows the deviation from 1.0 versus $x$ calculated for each $\bar{d} / \bar{u}$ point of the SeaQuest result. Deviation is defined as $(\bar{d} / \bar{u}-1) / \sigma_{\text {stat. }}$, showing the statistical significance of the deviation from 1.0. The figure indicates that the $\bar{d} / \bar{u}$ of the SeaQuest result is clearly higher than 1 at $0.1<x<0.45$, in contrast to the E866 result. For the last point at $0.45<x<0.58$, the result shows that the ratio is close to unity. It should be noticed that nuclear effect of deuterium [49, 50] is not taken into account at the moment. This effect on the flavor asymmetry is being investigated. 

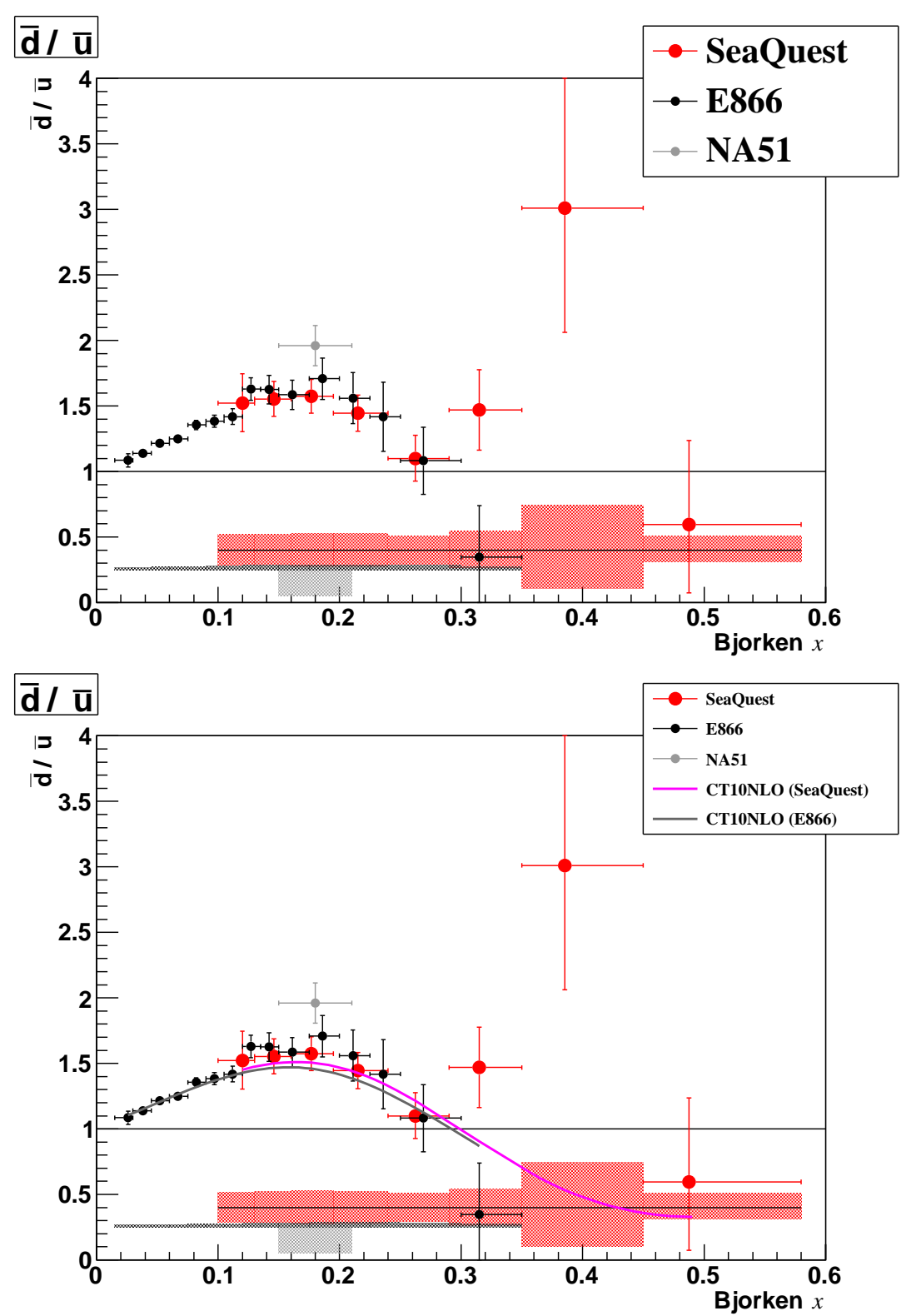

Figure 6.5: $\bar{d} / \bar{u}$ ratio as a function of Bjorken $x$. The SeaQuest result is plotted in red and the E866 result in black, and the NA51 result in gray. The error bars represent the statistical uncertainty, and the error band at bottom represent the systematic uncertainty. The position of the error band was arbitrary chosen. In the bottom figure, also the predictions calculated with CT10NLO PDF parameterizations for SeaQuest (magenta line) and E866 (gray line) are drawn. 


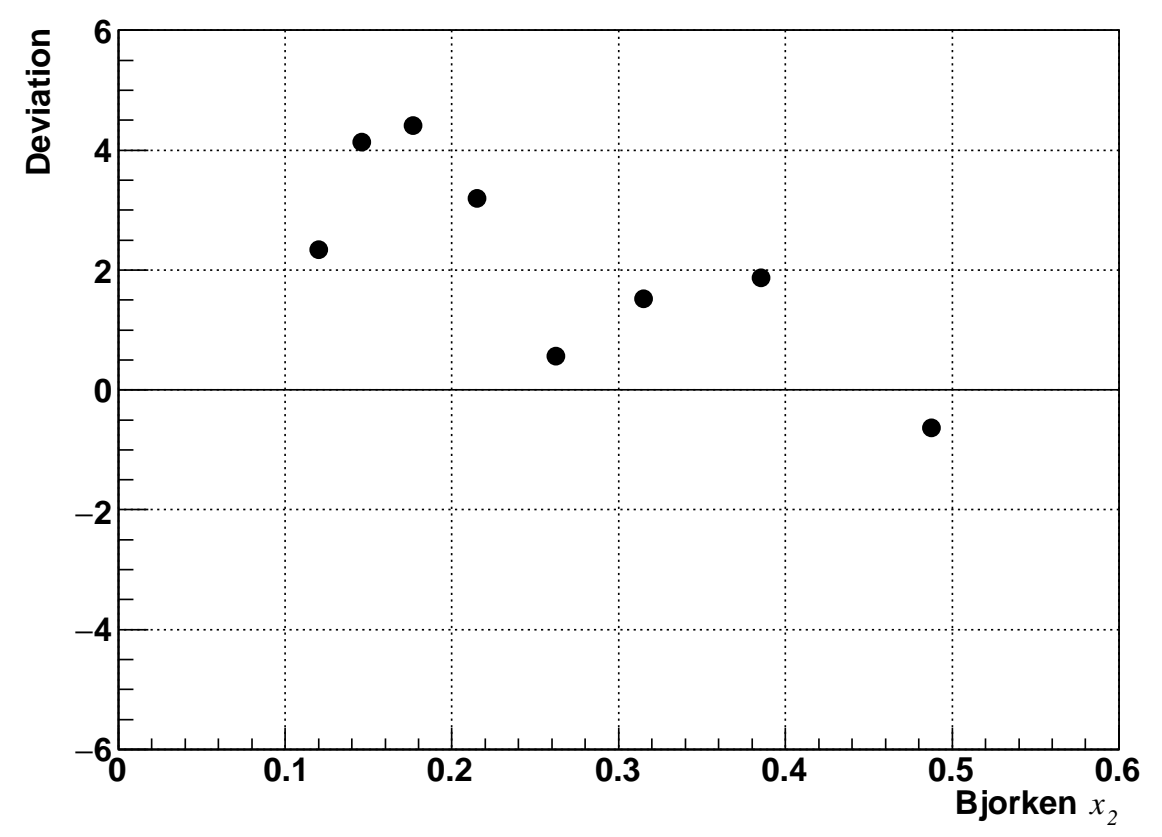

Figure 6.6: Deviation defined as $(\bar{d} / \bar{u}-1) / \sigma_{\text {stat. }}$, versus $x$ calculated for each $\bar{d} / \bar{u}$ point of SeaQuest. The Deviation shows the statistical significance of the deviation from 1.0. 


\subsubsection{PDF parameterizations}

The SeaQuest result is compared also with predictions using several PDF parameterizations in Fig. 6.7: CT10NLO[48], CT14NLO[5]] and MMHT2014NLO[52]. These three are shown to be in good agreement with the SeaQuest result at $0.1<x<0.3$, while they behave differently at $x=0.3$ and higher. The CT10NLO calculated shortly after

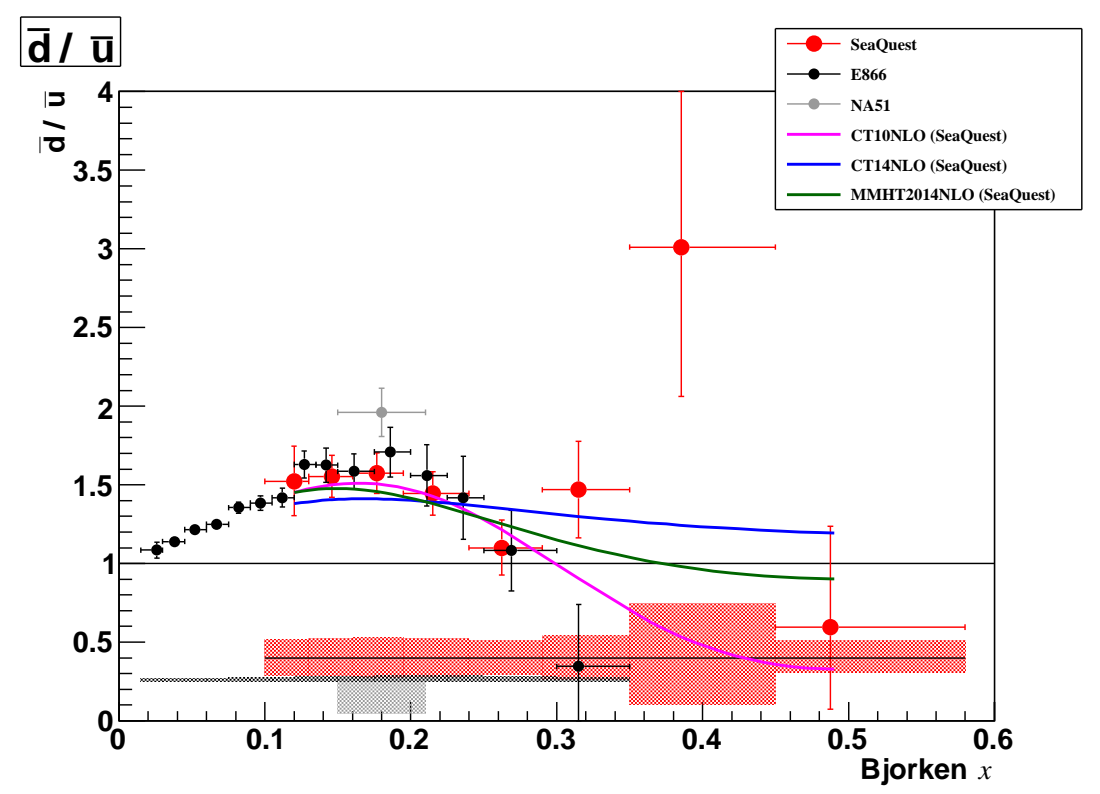

Figure 6.7: $\quad \bar{d} / \bar{u}$ result with the predictions using PDF parameterizations: CT10NLO in magenta, CT14NLO in blue, and MMHT2014NLO in green.

the E866 result was released is strongly affected by the E866 result, namely the ratio of $\bar{d} / \bar{u}$ goes less than one at $x=0.3$ and higher. This trend has been revised; $\bar{d} / \bar{u}$ becomes higher, even more than one, at high $x$ region with newer PDF parameterizations. $\chi^{2}$ is calculated for those three predictions to know how close they are to the SeaQuest result, where $\chi^{2}$ is defined as:

$$
\chi^{2}=\sum \frac{\left\{(\bar{d} / \bar{u})_{x}-f(x)\right\}^{2}}{\sigma_{\text {stat. }}^{2}}
$$

where $f(x)$ is the curve of prediction with the PDF parameterizations. The results are listed in Table. 6.3. The conclusion that the prediction using the MMHT2014NLO parameterizations is closest to the SeaQuest result. It should be noted that CT10NLO parameterizations was used to extract the SeaQuest result. If PDF is updated in near future, it can be used in the iterative analysis of SeaQuest. 


\begin{tabular}{ll}
\hline \hline PDF & $\chi^{2}$ \\
\hline CT10NLO & 27.6 \\
CT14NLO & 15.5 \\
MMHT2014NLO & 14.0 \\
\hline
\end{tabular}

Table 6.3: Results of $\chi^{2}$ for the 8 data points for the predictions by the three PDF parameterizations.

\subsubsection{Possible origins of the sea quark in the proton}

\section{Perturbative origins}

The asymmetry of antiquark distributions in the proton was observed by the NMC experiment for the first time by testing the Gottfried sum rule. After the measurement, Field and Feynman suggested[54] that it could be due to Pauli blocking. The perturbative production of the sea quark in the proton is the gluon splitting, $g \rightarrow q+\bar{q}$. They suggested that $u \bar{u}$ pairs would be suppressed relative to $d \bar{d}$ pairs in the production because of the presence of two $u$ valence quarks in the proton as compared to a single $d$ valence quark.

However, after the suggestion, it turned out that the effect of the Pauli blocking to the flavor asymmetry in the proton was less than one percent [55, 56]. Because of that, another, probably non-perturbative, mechanism must be found to account for the larger measured $\bar{d}, \bar{u}$ asymmetry.

\section{Non-perturbative origins}

Pion-cloud models [57, 58, 5.9] describe the non-perturbative production of sea quarks in the proton, and it was suggested that this model can express the flavor asymmetry of antiquarks. In this model, the proton is dissociated into virtual $N+\pi$ or $\Delta+\pi$. So, pions surround the bare proton like "cloud". The proton can be expressed as

$$
\begin{aligned}
|p\rangle= & \sqrt{1-|\alpha|^{2}-|\beta|^{2}}\left|p_{0}\right\rangle \\
& +\alpha\left[\sqrt{2 / 3}\left|p+\pi^{0}\right\rangle-\sqrt{1 / 3}\left|n+\pi^{+}\right\rangle\right] \\
& +\beta\left[\sqrt{1 / 2}\left|\Delta^{++}+\pi^{-}\right\rangle-\sqrt{1 / 3}\left|\Delta^{+}+\pi^{0}\right\rangle+\sqrt{1 / 6}\left|\Delta^{0}+\pi^{+}\right\rangle\right]
\end{aligned}
$$

where $\left|p_{0}\right\rangle$ is the bare proton with a symmetric sea quark that includes contributions from perturbative processes $(g \rightarrow u \bar{u}, d \bar{d})$, and $|\alpha|^{2}$ and $|\beta|^{2}$ are the probabilities that the proton is in a virtual $N+\pi$ or $\Delta+\pi$ state, respectively. Since the valence quarks in $\pi^{+}$are $u \bar{d}$ while they are $d \bar{u}$ in $\pi^{+}$, the observed flavor asymmetry (excess of $\bar{d}$ than $\bar{u}$ ) in the proton can be expressed by the dominance of the production of virtual $\pi^{+}$ 
states compared to that of virtual $\pi^{-}$states. The $|p\rangle$ state can be varied by cut off parameter and coupling constants between meson and baryon. A prediction curve of the pion model[5.9] is drawn in Fig. 6.8 .

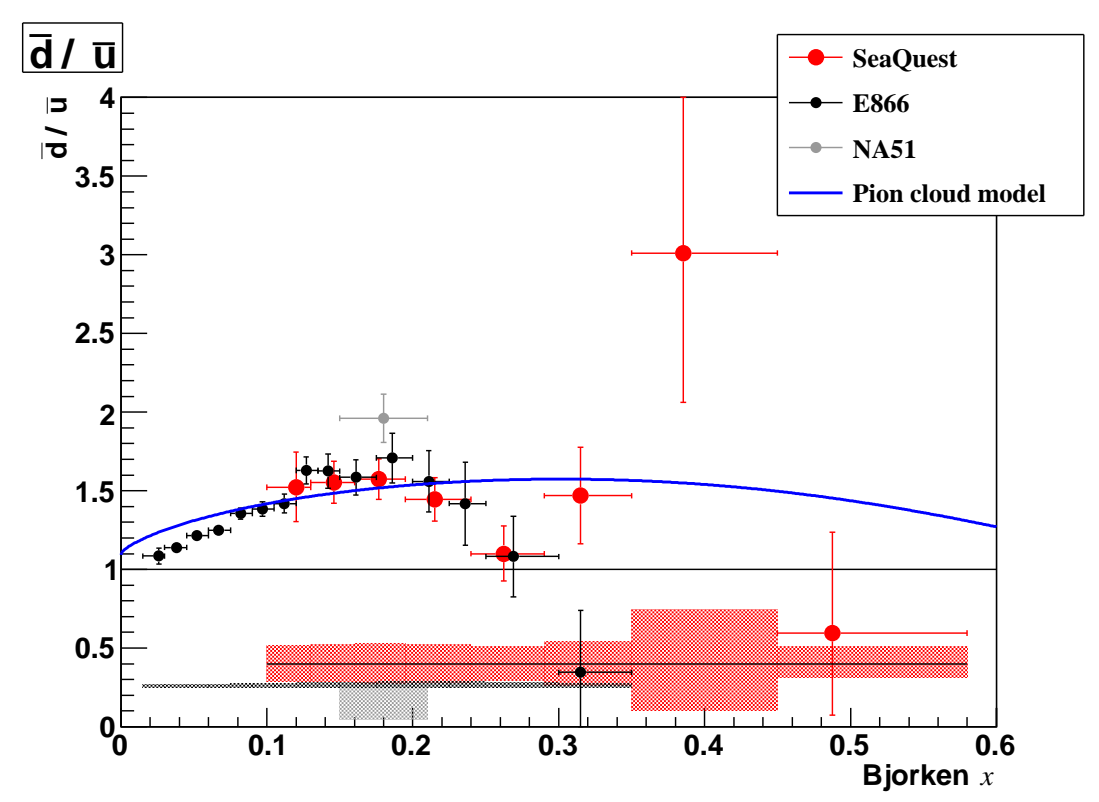

Figure 6.8: Comparison of the measured results with the prediction of pion-cloud model[5.9]. The points and error bars show the $\bar{d} / \bar{u}$ results obtained in measurements like the other figures, and the blue line shows the prediction of the pion-cloud model.

The $\chi^{2} /$ ndf, where $\chi^{2}$ is defined in Eq. 6.7 and $f(x)$ in the equation is the curve of prediction by the pion-cloud model, is calculated for both SeaQuest and E866 results to know how close these results to the curve by the pion-cloud model. The value is 3.7 for the SeaQuest result and 7.7 for the E866 result. So, the model with parameters in [5.9] agrees better with the SeaQuest result than the E866 result. 


\section{Chapter 7}

\section{Conclusion and Future Prospect}

\subsection{Conclusion}

The SeaQuest (E906) experiment studies the internal structure of the proton at Fermi National Accelerator Laboratory (Fermilab). One of the goals of the experiment is to measure the flavor asymmetry between $\bar{d}$ and $\bar{u}$ in the proton as a function of Bjorken $x$. The first result of the flavor asymmetry using the SeaQuest data is extracted and presented in this thesis.

The SeaQuest detects dimuons from Drell-Yan process in the $p$ - $p$ or $p$ - $d$ reactions using the $120 \mathrm{GeV}$ proton beam from the Main Injector at Fermilab. The Drell-Yan process takes place in hadron-hadron collisions when a quark in one hadron in the beam and an antiquark in other hadron in the target annihilate into a virtual photon that decays into a lepton pair. The process is well suited to measure the anti-quark distributions of the proton since an anti-quark is always involved in this process.

The conclusions of this thesis are following:

- The SeaQuest spectrometer for dimuons was constructed and all the detectors were installed. Data were smoothly accumulated.

- The SeaQuest data taken during the run II and III are analyzed. The data contains $\sim 3.3 \times 10^{4}$ spills, where a spill is a 5-second-long beam time. In total we received $\sim 9.4 \times 10^{17}$ protons during this period. Roughly half of them are "live protons" to be analyzed, then $\sim 80 \%$ of the data by the live protons passed spill cuts and are analyzable data.

- Reconstruction of charged particle is done, and dimuons are obtained. Their kinematics is good and is in agreement with the design. The Detectors and DAQ system worked well. 
- About 40000 of dimuon events are obtained for $\bar{d} / \bar{u}$ analysis for LH2 and LD2 targets in total ( 20000 events for LH2 and also $\sim 20000$ events for LD2 target).

- The performance of the drift chambers are checked, and it is confirmed that they work fine.

- The flavor asymmetry between $\bar{d}$ and $\bar{u}$ as a function of Bjorken $x$ was extracted using the SeaQuest data for the first time. It was extracted for much wider Bjorken $x$ region than the previous experiment. The measured Bjorken $x$ range covers up to 0.58 .

- The result shows that the ratio of $\bar{d} / \bar{u}$ is always higher than 1 at $0.1<x<0.45$, in contrast to the E866 result. For $0.45<x<0.58$, the result shows that the ratio is close to unity.

- Prediction obtained by one of the non-perturbative models, Pion-cloud model[5.9], is closer to the SeaQuest result than the E866 result.

- Explaining the observed flavor asymmetry of anti-quarks in the proton with the QCD based on the first principle such as lattice Gauge calculation is important. If QCD fails to reproduce the experimental result, a new physics will be required.

\subsection{Future prospect}

Part of data taken in Run II and Run III are used for the analysis in this thesis. Meanwhile SeaQuest experiment is taking data in Run IV and will take data in Run V starting later

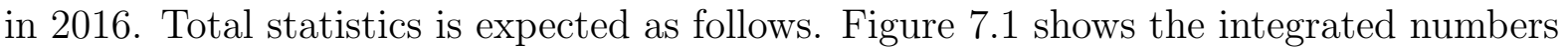
of protons on the SeaQuest targets as a function of time. The black line indicates the integrated number of delivered protons, the blue line indicates the integrated number of not-inhibited protons, and the red line indicates the integrated number of recorded protons. The delivered protons is just the delivered protons on the SeaQuest targets from Fermilab Main Injector regardless of the beam quality and the condition of the SeaQuest hardware and software. The not-inhibited protons are the protons not inhibited by QIE VETO, in other words, good-quality protons (no intensity spikes). The recorded protons are the protons not-inhibited and received when DAQ is live. Since the beam quality became better at Run III than Run II, the number of received protons per time increased (the slope increased). The slope slightly decreased at the beginning of Run IV because of drift chamber maintenance, and the slope recovered after the work finished. The detailed 


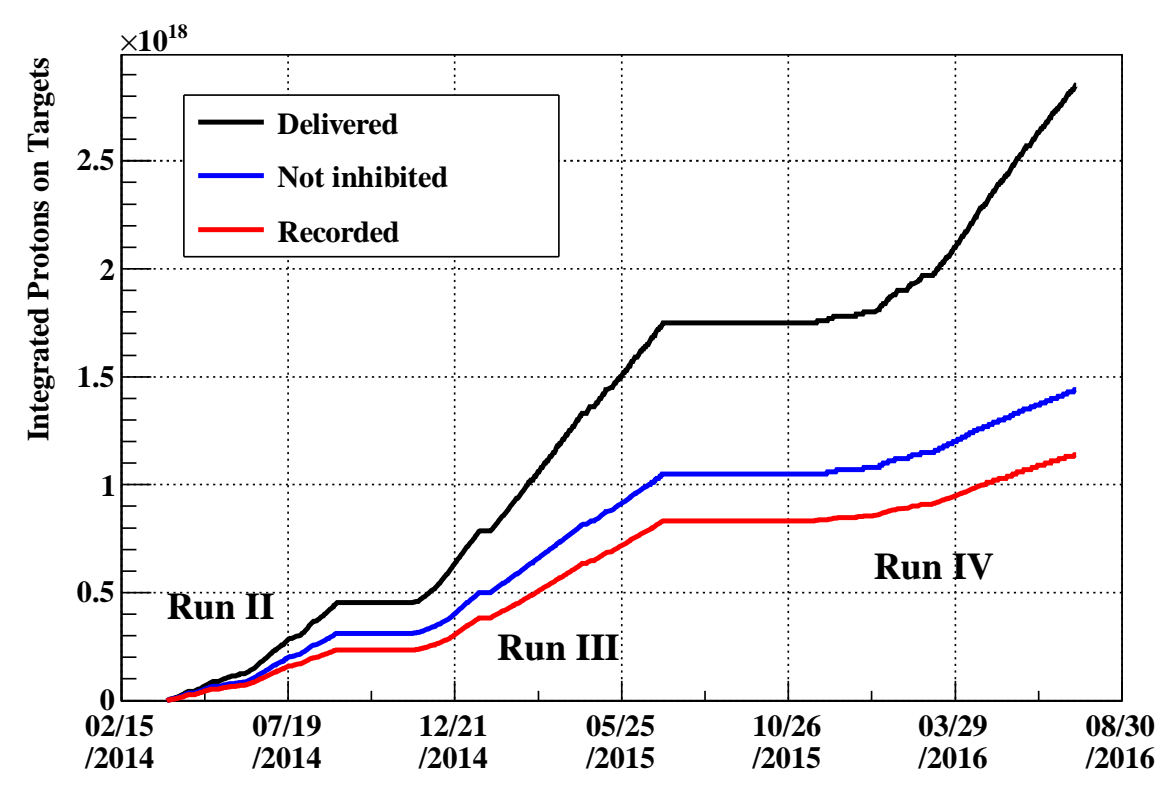

Figure 7.1: The integrated numbers of protons on the SeaQuest targets as a function of time. The black line indicates the integrated number of delivered protons, the blue line indicates the integrated number of not-inhibited protons, and the red line indicates the integrated number of recorded protons.

number of protons are listed in Table. 7.T. The numbers for Run $\mathrm{V}$ is expected values from the values of Run IV. For now, it is expected that $4.08 \times 10^{18}$ protons will be received, and $1.48 \times 10^{18}$ protons will be recorded in total while the proposed number of recorded protons is $3.4 \times 10^{18}$ [15]. That means roughly a half of the proposed number will be recorded by the end of Run $\mathrm{V}$.

\begin{tabular}{llll}
\hline \hline Run & & \multicolumn{2}{c}{ PoT } \\
& Delivered & Not Inhibited & Recorded \\
\hline Run II & $4.55 \mathrm{E}+17$ & $3.12 \mathrm{E}+17$ & $2.34 \mathrm{E}+17$ \\
Run III & $1.29 \mathrm{E}+18$ & $7.36 \mathrm{E}+17$ & $5.98 \mathrm{E}+17$ \\
Run IV & $1.10 \mathrm{E}+18$ & $3.92 \mathrm{E}+17$ & $3.07 \mathrm{E}+17$ \\
Run V & $1.23 \mathrm{E}+18$ & $4.38 \mathrm{E}+17$ & $3.43 \mathrm{E}+17$ \\
\hline Total & $4.08 \mathrm{E}+18$ & $1.88 \mathrm{E}+18$ & $1.48 \mathrm{E}+18$ \\
\hline
\end{tabular}

Table 7.1: The number of delivered, not-inhibited and recorded protons on the SeaQuest targets. The numbers for Run $\mathrm{V}$ is expected values from the values of Run IV. 


\subsection{Summary}

The result of $\bar{d} / \bar{u}$ asymmetry at wider Bjorken $x$ region, $0.1<x<0.58$, is useful information to understand the inner structure of the proton and the origin of the sea quarks in the proton. The result also contributes to improve the precision of PDF of sea quarks. This is important because the PDFs are used as an input to the simulations of various hadron reactions. 


\section{Acknowledgements}

I would like to express my deep gratitude to my supervisor Prof. Toshi-Aki Shibata. His guidance on detector construction and data analysis, and his advice on my doctor thesis were essential. He provided me with the opportunity to participate in the SeaQuest experiment at Fermi National Accelerator Laboratory and to study at the frontier of the particle physics. I am also deeply grateful to Prof. Kenichi Nakano for his valuable help and discussions. His advice concerning both hardware and software was very thoughtful.

I would like to thank all Japanese staff members of the SeaQuest collaboration who worked together for the drift chambers: Dr. Shin'ya Sawada, Dr. Yuji Goto and Dr. Yoshiyuki Miyachi. They gave me fruitful advice and discussions.

I would like to thank Dr. Paul E. Reimer and Dr. Don Geesaman, co-spokes-persons of the SeaQuest experiment. I thank them for their supports. I also would like to thank Dr. David Christian who supported me during my stay at Fermilab which is about three years all together. Also, his advice for constructing and maintaining detectors was fruitful. I like to thank Dr. Chuck Brown. He has a great thought for the construction of experiment, hardware, and analysis. Discussion with him was always useful.

I would like to thank all members of the $\bar{d} / \bar{u}$ analysis team: Prof. Kenichi Nakano, Bryan Kerns, Kei Nagai, Dr. Markus Diefenthaler, Dr. Paul E. Reimer and Dr. Naomi Makins. Discussions with them were productive and indispensable. I like to especially thank Bryan Kerns who worked for the analysis together. I would also like to thank Dr. Jen-Chieh Peng. He gave me lots of advice about physics analysis, especially pion-cloud model.

My physics analysis would not have been succeeded without the support of Dr. Kun Liu and Dr. Joshua Rubin. I would like express great gratitude to them. Kun and Josh have constructed the SeaQuest tracking tool to make the data analyzable. Their help to my software work was fruitful. I also like to thank Bryan Dannowitz, Evan McClellan, Bryan Ramson, Shivangi Prasad and Mae Hwee Teo. They also provided me a great advice and discussions for my analysis.

I would like to thank Dr. Lamiaa El Fassi. We have worked for the drift chambers 
together. All the chamber work was done with her great help. The construction of Station 3 minus drift chamber was done with many collaborator's help. I would like to thank all of them including the ACU students. I would like to thank Wanda Newby and David Northacker for their technical supports for the drift chamber. They gave us helpful advice on hardwares. I also like to thank Arun Tadepalli and Po-Ju lin. We worked together on constructing and maintaining the drift chambers as a chamber group.

I would like to thank Dr. Kazutaka Nakahara and Dr. Su-Win Grass Wang. They help me understand DAQ and trigger systems. It was very important to understand all the SeaQuest hardware and software system. Especially, Kaz was the only person who speaks both Japanese and English and helped me during my stay at Fermilab. stated studying in Fermilab.

It was a great pleasure to study and work together with Dr. Florian Sanftl. I was always surprised by his new ideas, and learned a lot of things from him. He is a great Physics and English teacher for me. Also, we had good time a lot in Chicago and in Tokyo. I would like express great my gratitude to him.

I like to thank all Japanese students of the SeaQuest collaboration, Shintaro Takeuchi, Kei Nagai, Shigeki Obata, Yuya Kudo and Shumpei Nara for their helps. It was a great experience to work and study together with them.

I like to thank all the Shibata lab members who studied with me together. I like to thank Koji Igarashi, Yasufumi Kunisada, Suguru Tamamushi, Takuto Miyazaki, Wataru Saito and Rui Sanada for discussions. It was a pleasure to discuss with them.

Finally, I would like to express my great gratitude to my parents for letting me study for long time and always supporting me in my life. 


\section{Bibliography}

[1] B. Povh, K. Rith, C. Scholz, F. Zetsche, "Particles and Nuclei: An Introduction to the Physical Concepts", Springer (1993).

[2] F. Halzen and A.D. Martin, "Quarks and Leptons: An introductory course in modern particle physics", Wiley (1984).

[3] D.H. Perkins, "Introduction to High Energy Physics" (1972).

[4] E.D.Bloom et al., Phys. Rev. Lett. 23, 930 (1969).

[5] M. Breidenbach et al., Phys. Rev. Lett. 23, 935 (1969).

[6] R.P. Feynman, Phys. Rev. Lett. 23, 1415 (1969).

[7] D.J. Gross and F. Wilczek, Phys. Rev. Lett. 30, 1343 (1973).

[8] P. Amaudruz et al. (New Muon), Phys. Rev. Lett. 66, 2712 (1991).

[9] M. Arneodo et al. (New Muon), Phys. Rev. D50, 1 (1994).

[10] A. Baldit et al., Phys. Lett. B 332, 244 (1994).

[11] S.D. Drell and T.M. Yan, Phys. Rev. Lett 25, 316 (1970).

[12] E.A. Hawker et al. (FNAL E866/NuSea), Phys. Rev. Lett. 80, 3715 (1998), hepex/9803011.

[13] J.C. Peng et al. (FNAL E866/NuSea), Phys. Rev. D58, 092004 (2001), hep$\mathrm{ph} / 9804288$.

[14] R.S. Towell et al. (FNAL E866/NuSea), Phys. Rev. D64, 052002 (2001), hepex/0103030.

[15] J. Arrington, et al. "Drell-Yan Measurement of Nucleon and Nuclear Structure with the Fermilab Main Injector: E906", SeaQuest proposal (2006). 
[16] M. Gell-Mann, Phys. Lett. 8, 214 (1964).

[17] G. Zweig, CERN Reports No. 8182/Th.401 and No. 8419/Th.412, 1964 (unpublished).

[18] H.W. Kendall, Rev. Mod. Phys., Vol.63, No.3, July (1991).

[19] Particle Data Group. http://pdg.lbl.gov/.

[20] V.N. Gribov, L.N. Lipatov, Sov. J. Nucl. Phys. B47, 365 (1973).

[21] Yu. L. Dokshitzer, Sov. Phys. JETP 46, 641 (1977).

[22] G. Altarelli and G. Parisi, Nucl. Phys. B126, 298 (1977).

[23] MSTW, A.D. Martin et al., Eur. Phys. J. C63, 189(2009).

[24] H.L. Lai et al.,, Phys. Rev. D55, 1280 (1997).

[25] H.L. Lai et al.,, Eur. Phys. J. C12, 375 (2000).

[26] The Fermilab Main Injector Technical Design Handbook, Fermi National Accelerator Laboratory, 1994.

[27] A Data Analysis Framework. http://root.cern.ch/.

[28] K. Nakahara. "Target schematics", SEAQUEST-doc-520-v1, 2012.

[29] Private communication with Tom O'Connor.

[30] The VME bus (VERSAModule Eurocard) architecture. http://www.caen.it/csite/CaenProd.jsp? parent=11\&idmod=705.

[31] W.M. Bokhari, J.G. Heinrich, N.S. Lockyer, and F.M. Newcomer. The ASDQ ASIC [Central Outer Tracker CDF upgrade application], pages 445446. Institute of Electrical and Electronics Engineers, 1998.

[32] Simulation of the passage of particles through matter. http://geant4.web.cern.ch/geant4/.

[33] M.H. Schub, et al., Phys Rev. D52, 1307 (1995).

[34] J. Pumplin, et al., JHEP, 012, 0207, (2002). 
[35] Jefferson Lab Data Acquisition Group. The coda data acquisition system. http://coda.jlab.org.

[36] S. Obata, Master thesis, Tokyo Institute of Technology (2014).

[37] http://pdg.lbl.gov/2014/AtomicNuclearProperties/.

[38] E. McClellan "kTracker and hardware Rate dependencies", SEAQUEST-doc-1554v1, 2016.

[39] B. Kerns, "Rate dependence update", SEAQUEST-doc-1608-v1, 2016.

[40] K. Nagai, "Correction of Chamber Plane Resolution", SEAQUEST-doc-1686-v2, 2016.

[41] L. Kun, "First draft of recommended analysis cuts for R005 production", SEAQUEST-doc-1487-v1, 2015.

[42] R. Towell, Ph. D. thesis, The University of Texas at Austin (1999).

[43] S. Miyasaka, "Study of effect if x2 migration on cross section ratio", SEAQUESTdoc-1246-v1, 2015.

[44] B.G.Tice and D.C.Christian, "Absolute Normalization - Beam Inhibit and DAQ Deadtime", SEAQUEST-doc-1212-v3, 2014.

[45] B. Kerns, "Rate dependence Summary Feb 2016", SEAQUEST-doc-1572-v3, 2016.

[46] D. C. Christian, SeaQuest Logbooks, 12090 (2016).

[47] F. Sanftl, Ph. D. thesis, Tokyo Institute of Technology (2014).

[48] H. Lai, et al., Phys Rev. D82, 074024 (2010).

[49] D.M. Alde, et al., Phys. Rev. Lett. 64, 2479 (1990).

[50] M.A. Vasiliev, et al., Phys. Rev. Lett. 83, 2304 (1999).

[51] S. Dulat, et al., Phys. Rev. D93, 033006 (2016).

[52] L.A. Haland-Lang, A.D. Martin, P. Motylinski, and R.S. Thorne, Eur.Phys.J. C75, 204 (2015).

[53] A. Buckley, et al., Eur.Phys.J. C75 3, 132 (2015). 
[54] R.D. Field and R.P. Feynman, Phys. Rev. D15, 2590 (1977).

[55] D.A. Ross and C.T. Sachrajda, Nucl. Phys. B149, 497 (1979).

[56] F.M. Steffens and A.W. Thomas, Phys. Rev. C55, 900 (1997).

[57] S. Kumano, Phys. Rev. D43, 3067 (1991).

[58] S. Kumano, Phys. Rep. 303, 183 (1998).

[59] J.C. Peng et al., Phys. Rev. D58, 092004 (1998).

[60] J.C. Peng et al., arXiv:hep-ph/0007341 (2000). 